Christian Haslbeck

\title{
Zentrale versus dezentrale Internalisierung externer Effekte bei unvollständiger Information
}




\section{Christian Haslbeck}

\section{Zentrale versus dezentrale Internalisierung externer Effekte bei unvollständiger Information}

Können externe Effekte in Gegenwart unvollständiger Information über ihre Nutzen und Kosten im Rahmen privater Verhandlungen vollständig internalisiert werden, oder sollte besser der Staat für eine effiziente Ressourcenallokation sorgen? Die vorliegende Arbeit versucht, mit Hilfe neuerer informationsökonomischer Methoden Antworten auf diese Frage zu geben. Es zeigt sich, daß zentrale Regulierungsmaßnahmen gerade vor dem Hintergrund des Informationsproblems an Bedeutung gewinnen.

Christian Haslbeck wurde 1964 in Frontenhausen geboren. Er studierte von 1984 bis 1990 Volkswirtschaftslehre an der Universität Regensburg. Seit 1990 ist er Wissenschaftlicher Mitarbeiter am Lehrstuhl für Finanzwissenschaft der Universität Regensburg und promovierte dort 1994 zum Dr. rer. pol. 
Zentrale versus dezentrale Internalisierung externer Effekte bei unvollständiger Information 


\section{FINANZWISSENSCHAFTLICHE SCHRIFTEN}

Herausgegeben von den Professoren

Albers, Krause-Junk, Littmann, Oberhauser, Pohmer, Schmidt

Band 68

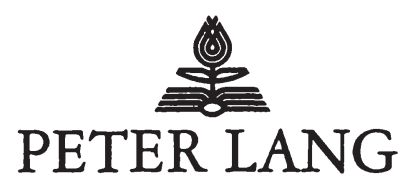

Frankfurt am Main - Berlin · Bern . New York · Paris . Wien 


\title{
Christian Haslbeck
}

\author{
Zentrale versus dezentrale \\ Internalisierung externer Effekte \\ bei unvollständiger Information
}

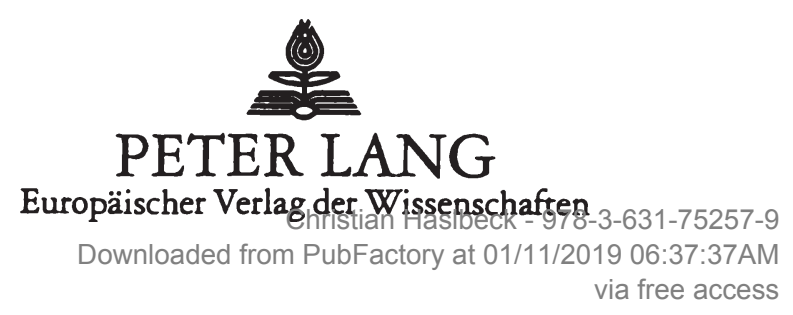


Die Deutsche Bibliothek - CIP-Einheitsaufnahme

Haslbeck, Christian:

Zentrale versus dezentrale Internalisierung externer Effekte bei unvollständiger Information / Christian Haslbeck. - Frankfurt am Main ; Berlin ; Bern ; New York ; Paris ; Wien : Lang, 1995

(Finanzwissenschaftliche Schriften ; Bd. 68)

Zugl.: Regensburg, Univ., Diss., 1994

ISBN 3-631-47944-1

NE: GT

Open Access: The online version of this publication is published on www.peterlang.com and www.econstor.eu under the international Creative Commons License CC-BY 4.0. Learn more on how you can use and share this work: http://creativecommons. org/licenses/by/4.0.

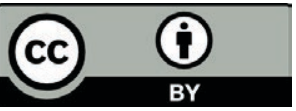

This book is available Open Access thanks to the kind support of ZBW - Leibniz-Informationszentrum Wirtschaft.

\author{
D 355 \\ ISSN 0170-8252 \\ ISBN 3-631-47944-1 \\ ISBN 978-3-631-75257-9 (eBook) \\ (C) Peter Lang $\mathrm{GmbH}$ \\ Europäischer Verlag der Wissenschaften \\ Frankfurt am Main 1995 \\ Alle Rechte vorbehalten.
}

Das Werk einschließlich aller seiner Teile ist urheberrechtlich geschützt. Jede Verwertung außerhalb der engen Grenzen des Urheberrechtsgesetzes ist ohne Zustimmung des Verlages unzulässig und strafbar. Das gilt insbesondere für Vervielfältigungen, Übersetzungen, Mikroverfilmungen und die Einspeicherung und Verarbeitung in elektronischen Systemen. 


\section{Für Teresa}

Christian Haslbeck - 978-3-631-75257-9

Downloaded from PubFactory at 01/11/2019 06:37:37AM

via free access 
Christian Haslbeck - 978-3-631-75257-9

Downloaded from PubFactory at 01/11/2019 06:37:37AM

via free access 


\section{Vorwort}

Eine der wichtigsten Aufgaben der finanzwissenschaftlichen Allokationstheorie besteht in der Begründung der Notwendigkeit staatlicher Eingriffe in den Marktprozeß. Kaum bestritten ist dabei die Auffassung, da ein Staatseingriff immer dann geboten ist, wenn Marktversagen vorliegt, d.h. wenn das freie Spiel der Marktkräfte nicht zu einer pareto-optimalen Allokation der Ressourcen führt. Die Geister scheiden sich jedoch, sobald es um die Frage geht, wann der Markt tatsächlich versagt.

$\mathrm{Da} B$ diese Frage nicht ohne weiteres zu beantworten ist, hat in der Vergangenheit v.a. die Diskussion um den geeigneten Weg zur Internalisierung externer Effekte gezeigt. Während für A. C. Pigou (1932) kein Zweifel daran bestand, daß Externalitäten zu Fehlallokationen führen, die nur durch einen Staatseingriff korrigiert werden können, brachte R. Coase (1960) diese Position stark ins Wanken, indem er auf die Möglichkeit einer vollständigen Internalisierung durch freiwillige Verhandlungen zwischen den betroffenen Parteien hinwies. Man kann wohl mit einigem Recht behaupten, da $\beta$ es seit Coase schwierig geworden ist, unter bestimmten Rahmenbedingungen Staatseingriffe zur Internalisierung externer Effekte zu begründen. $\mathrm{Zu}$ diesen Rahmenbedingungen gehört $\mathrm{u}$. a. vollständige Information über Kosten und Nutzen, die mit der Ausübung der Externalität verbunden sind. Ist diese Voraussetzung gegeben, besteht - zumindest bei Externalitätsproblemen mit einer geringen Anzahl von Beteiligten - kaum Anlaß, an der Effizienz privater Internalisierungsverhandlungen zu zweifeln. Die Intervention einer Zentralinstanz wäre somit günstigstenfalls überflüssig.

Nun ist aber die Annahme vollständiger Information zweifelsohne mehr als heroisch. Eine interessante Fragestellung ist deshalb, wie private Verhandlungen im Vergleich zu Staatseingriffen bei unvollständiger Information abschneiden. Intuitiv würde man wohl vermuten, daß sich unter diesen Umständen die Vorteilhaftigkeitsrelationen noch stärker zugunsten einer dezentralen Lösung verschieben. Das Hauptanliegen der vorliegenden Arbeit ist es zu untersuchen, inwieweit sich diese Vermutung bestätigt oder nicht.

Die Arbeit entstand während meiner Tätigkeit als Wissenschaftlicher Mitarbeiter von Prof. Dr. Wolfgang Buchholz am Institut für Volkswirtschaftslehre einschließlich Ökonometrie der Universität Regensburg. Sie wurde im Mai 1994 von der Wirtschaftswissenschaftlichen Fakultät der Universität als Dissertation angenommen. 
Ich möchte es an dieser Stelle nicht versäumen, all denjenigen zu danken, die, direkt oder indirekt, zum Gelingen dieser Arbeit beigetragen haben. In erster Linie gilt mein Dank Wolfgang Buchholz, der unter ausgezeichneten Arbeitsbedingungen meine Promotion mit seiner ständigen Diskussionsbereitschaft und seinem unermüdlichen Engagement förderte und mir nicht selten auch in außerfachlichen Belangen mit Rat und Tat zu Seite stand. Wolfgang Wiegard danke ich für seine Mitarbeit in der Betreuungskommission und die tatkräftige Förderung meiner akademischen Laufbahn an der Universität Regensburg. Desweiteren danke ich meinen Freunden und Kollegen Max Frank, Claudia Löhnig und Franz Seitz, die mich, sei es durch ihre Diskussionsbeiträge, sei es durch ihre freundliche Aufmunterung in gelegentlichen "Schaffenskrisen", unterstützten. Nicht zuletzt danke ich meiner Frau Gertraud, die das Manuskript mit großer Sorgfalt korrekturgelesen hat und mir den zuweilen dringend nötigen moralischen Beistand leistete. 


\section{Inhalt}

1. Einleitung: Überblick über die traditionelle Pigou-Coase-Kontroverse ........ 1

1.1 Der Pigou-Ansatz............................................................................... 2

1.2 Der "Property-Rights"-Ansatz von Coase ........................................................ 5

1.3 Hauptargumente der Pigou-Coase-Kontroverse ............................................ 8

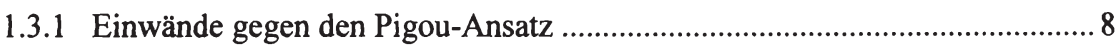

1.3.2 Einwände gegen den Property-Rights-Ansatz ........................................... 11

1.4 Unvollständige Information: Ein Problem in beiden Ansätzen ........................ 15

1.5 Zielsetzung und Aufbau der Arbeit.............................................................. 18

Teil I: Die Internalisierung externer Effekte im Rahmen nichtkooperativer Verhandlungen ............................................................................... 21

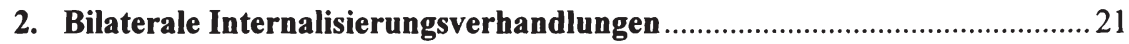

2.1 Begründung der Notwendigkeit nicht-kooperativer Verhand-

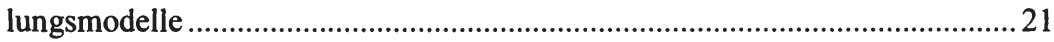

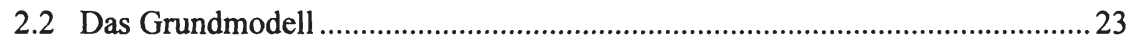

2.3 Ein einfaches Verhandlungsmodell mit vollständiger Information ...................25

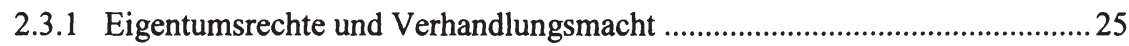

2.3.2 Die Verhandlungslösung bei vollständiger Information..............................29

2.4 Das Verhandlungsmodell mit unvollständiger Information ............................ 33

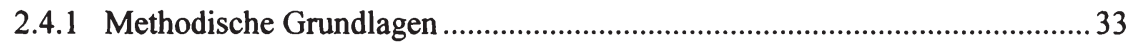

2.4.1.1 Die Grundstruktur des "Mechanism-Design"-Ansatzes ............................. 33

2.4.1.2 Das Enthüllungsprinzip und seine Implikationen .......................................36

2.4.1.3 Das Optimierungsproblem des Schädigers ................................................. 40

2.4.1.3.1 Die Monotonie der Outputfunktion ..................................................... 42

2.4.1.3.2 Die Bedingungen der individuellen Rationalität......................................4 44

2.4.1.3.3 Anreizverträglichkeit und Informationsrenten......................................... 45

2.4.2 Die Verhandlungslösung bei unvollständiger Information...........................51

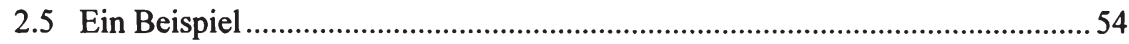

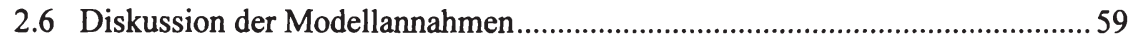

2.6.1 Rechtfertigung des "Mechanism-Design"-Ansatzes .......................................60 
2.6.2 Die Rolle der Verhandlungsmacht ............................................................61

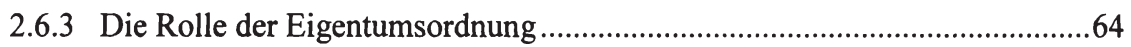

3. Internalisierungsverhandlungen mit mehreren Beteiligten .........................67

3.1 Problemstellung und Begründung der Modellwahl..........................................67

3.2 Erweiterung des Verhandlungsmodells auf mehrere Geschädigte.....................71

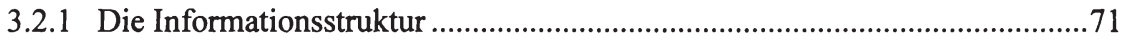

3.2.2 Bayesianische Gleichgewichte und Enthüllungsprinzip .............................72

3.2.3 Die Verhandlungslösung mit mehreren Geschädigten...................................76

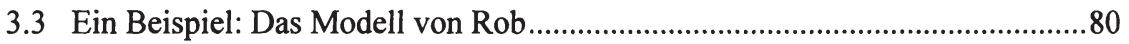

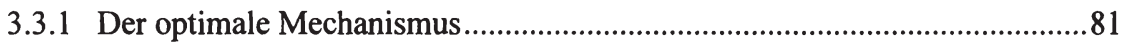

3.3.2 Graphische Veranschaulichung des Mechanismus ........................................84

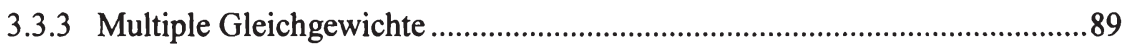

3.3.4 Zwei Maße zur Beurteilung der Effizienz des Mechanismus ........................94

3.3.5 Wohlfahrtseffekte bei Erhöhung der Anzahl der Geschädigten.....................97

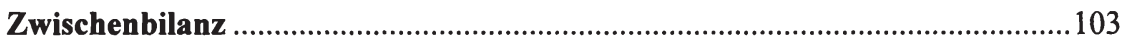

Teil II: Die Internalisierung externer Effekte durch eine Zentralinstanz...........107

4. Informationsprobleme beim Einsatz traditioneller Lenkungs-

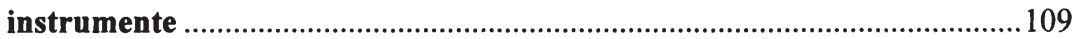

4.1 Strategisches Verhalten des Geschädigten bei Auflagen-

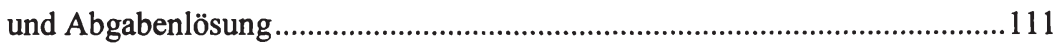

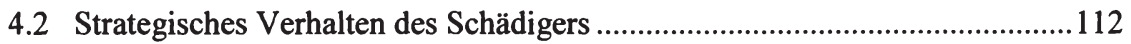

4.2.1 Übertreibung des Grenzgewinns bei der Auflagenlösung............................112

4.2.2 Untertreibung des Grenzgewinns bei der Abgabenlösung ...........................114

5. Mechanismen zur Präferenzenthüllung ......................................................119

5.1 Wahrheitsgemäße Offenbarung als dominante Strategie:

Die Klasse der Groves-Mechanismen ......................................................119

5.1.1 Charakterisierung der Groves-Mechanismen .............................................121

5.1.2 Schwächen der Groves-Mechanismen ....................................................124

5.1.2.1 Versagen in Gegenwart von Einkommenseffekten ...................................124 
5.1.2.2 Die Unmöglichkeit eines ausgeglichenen Budgets................................. 127

5.1.3 Versuche zur Rettung der Groves-Mechanismen ..................................... 135

5.2 Ausgeglichenes Budget statt dominanter Strategien:

Der Mechanismus von d'Aspremont und Gerard-Varet ................................ 137

5.2.1 Charakterisierung des AGV-Mechanismus .................................................. 139

5.2.2 Schwächen des AGV-Mechanismus ......................................................... 142

5.2.2.1 Schwächen des Gleichgewichtskonzepts ............................................... 143

5.2.2.2 Verteilungsprobleme: Fehlende individuelle Rationalität ........................ 145

5.3 Praktische Einwände gegen Präferenzenthüllungsmechanismen ................... 150

6. Einfache staatliche Internalisierungsinstrumente im Vergleich zur

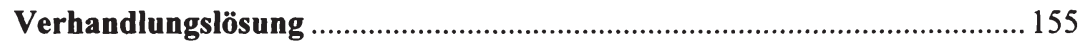

6.1 Eine praktikable Alternative: Durchschnittlich optimale

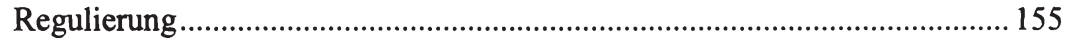

6.2 Second-Best-Vergleich zwischen privaten Verhandlungen

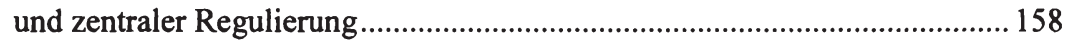

6.2.1 Preis- vs. Mengenregulierung bei bekannter Gewinnfunktion .................... 159

6.2.2 Preis- vs. Mengenregulierung bei unbekannter Gewinnfunktion ................. 161

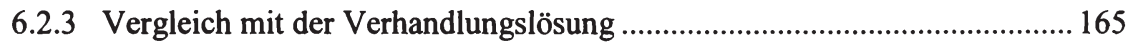

6.2.4 Synthese zwischen Verhandlungen und Staatseingriffen ........................... 170

Zusammenfassung und Bewertung der Ergebnisse........................................ 173

Anhänge

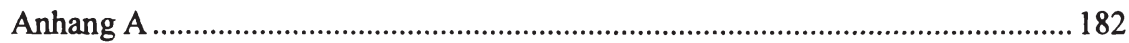

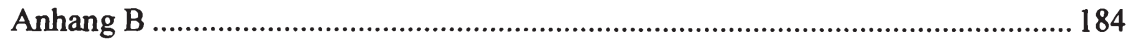

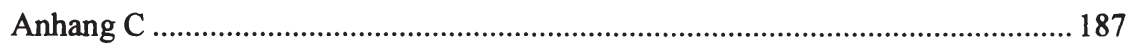

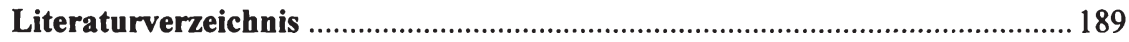


Christian Haslbeck - 978-3-631-75257-9

Downloaded from PubFactory at 01/11/2019 06:37:37AM

via free access 


\section{Einleitung: Überblick über die traditionelle Pigou-Coase-Kontroverse}

Unter den verschiedenen Ansätzen zur Begründung eines allokativen Marktversagens bei vollkommener Konkurrenz nimmt die Theorie externer Effekte zweifellos eine gewisse Sonderstellung ein. Seit Arthur C. Pigou in seinem erstmals 1920 erschienenen Werk "The Economics of Welfare" den Grundstein dieser Theorie legte, zählt das Externalitätsproblem zu den am häufigsten und kontroversesten diskutierten wohlfahrtsökonomischen Fragestellungen. Dafür spricht allein schon die Fülle von Literaturbeiträgen, die im Zusammenhang damit erschienen sind. Wenn man nach den Gründen für die bis heute anhaltende Popularität und Aktualität dieser Thematik fragt, so ist zunächst festzustellen, daß die Auseinandersetzung mit der Umweltverschmutzung als einem der drängendsten Probleme unserer Zeit innerhalb der Wirtschaftswissenschaften im wesentlichen auf der Theorie externer Effekte basiert. Die Umweltökonomie faßt seit den sechziger Jahren Umweltprobleme im wesentlichen als Konsequenzen nicht internalisierter externer Effekte auf. ${ }^{1}$ Abgesehen von dieser eher wirtschaftspolitischen Dimension liegt die Bedeutung des Externalitätsproblems aber auch in starkem Maße auf theoretischer Ebene. Dies ist dadurch begründet, daß die Frage nach den Möglichkeiten der Internalisierung externer Effekte eine sehr fruchtbare Grundsatzdiskussion zwischen den Vertretern zweier grundlegend unterschiedlicher Denkrichtungen auslöste: Während die Anhänger des sogenannten Pigou-Ansatzes zur Beseitigung von Fehlallokationen, die durch Externalitäten hervorgerufen werden, staatliche Interventionen befürworteten, hielten Ökonomen, die in der Coase-Tradition argumentierten, Staatseingriffe für übertrieben oder sogar schädlich. Dieser fast schon ideologische Streit, der bis in die Gegenwart andauert, hat im Laufe der Jahre zu einigen wichtigen Erkenntnissen geführt, deren Tragweite nicht allein auf das Externalitätsproblem beschränkt bleibt, sondern sich allgemein auf die Korrektur von Allokationsstörungen jeglicher Art erstreckt. Im folgenden soll ein kurzer Überblick über die Grundpositionen der Diskussionsgegner und ihre wichtigsten Argumente in dieser Kontroverse gegeben werden.

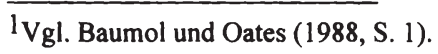




\subsection{Der Pigou-Ansatz}

Das Konzept externer Effekte wird von Pigou in Kapitel IX seiner "Economics of Welfare" entwickelt. ${ }^{2}$ Die Abschnitte, in denen er sich auf die Begründung eines Marktversagens selbst bei vollkommener Konkurrenz bezieht, können zusammengefaßt in etwa wie folgt wiedergegeben werden: Die Maximierung der gesamtwirtschaftlichen Wohlfahrt erfordert eine Übereinstimmung der sozialen Nettogrenzprodukte aller eingesetzten Ressourcen in ihren verschiedenen Verwendungen. Da sich nutzenmaximierende Individuen in ihren Entscheidungskalkülen aber an den privaten Nettogrenzprodukten ihrer ökonomischen Aktivitäten orientieren und der Marktprozeß bei vollkommenem Wettbewerb über den Preismechanismus für deren Angleichung sorgt, führt die Verfolgung individueller Eigeninteressen immer dann zu Fehlallokationen, wenn privates und soziales Nettogrenzprodukt einer Aktivität voneinander abweichen. Eine Möglichkeit solcher Divergenzen ist gegeben, wenn die Aktivität eines bestimmten Individuums positive oder negative Auswirkungen auf andere Individuen mit sich bringt, ohne daß für diese Effekte Zahlungen von den Nutznießern gefordert werden können bzw. Kompensationen an die Geschädigten gezahlt werden müssen. ${ }^{3}$ Die Wurzel des Problems externer Effekte liegt also mit anderen Worten im Fehlen von Preisen als Signale für ihre gesamten Nutzen oder Kosten. Unter diesen Umständen ist die Allokation der Ressourcen durch den Markt selbst unter den Bedingungen vollkommenen Wettbewerbs ineffizient. Pigous Folgerung aus dieser Erkenntnis bestand in dem Ruf nach Staatseingriffen zur Korrektur solcher Fehlallokationen: "It is, however, possible for the State, if it so chooses, to remove the divergence in any field by 'extraordinary encouragements' or 'extraordinary restraints' upon investment in that field. The most obvious forms which these encouragements and restraints may assume are, of course, those of bounties and taxes."4

Die Logik, die hinter dieser Überlegung steckt, ist einleuchtend: Wenn von bestimmten Aktivitäten mancher Agenten ökonomische Wirkungen ausgehen, für die der freie Markt keine Preise zustande bringt, dann muß eben der Staat in Form

\footnotetext{
2 Siehe Pigou (1932, S. 172 ff.).

${ }^{3}$ Diese Definition, die sinngemäß von Pigou selbst stammt (Vgl. Pigou (1932, S. 183)), entspricht in etwa dem, was die moderne Literatur heute unter externen Effekten versteht. Pigou nennt daneben noch zwei weitere Möglichkeiten der Divergenz zwischen sozialem und privatem Nettogrenzprodukt bei vollkommener Konkurrenz. (Vgl. Pigou (1932, S. 174 ff. bzw. S. 191 f. )).

${ }^{4}$ Siehe Pigou (1932, S. 192).
} 
von Subventionen oder Steuern "künstliche" Preise schaffen, die den Individuen die gesamtwirtschaftlichen Konsequenzen ihres Verhaltens aufzeigen und sie dazu anhalten, diese bei ihren einzelwirtschaftlichen Entscheidungen zu berücksichtigen. Dieser Grundgedanke, von Pigou selbst noch etwas diffus und interpretationsbedürftig formuliert ${ }^{5}$, wurde in der Folgezeit von zahlreichen Ökonomen aufgegriffen und so weit ausgearbeitet und verfeinert, daß auch die moderne Wohlfahrtsökonomie davon Gebrauch machen konnte.

Die Kernaussage, die sich im Laufe der Zeit aus den früheren Arbeiten zum Pigou-Ansatz herauskristallisierte, kann in ihrer einfachsten Form mit Hilfe eines Diagramms erläutert werden, das mittlerweile aus vielen Lehrbüchern wohlbekannt ist.

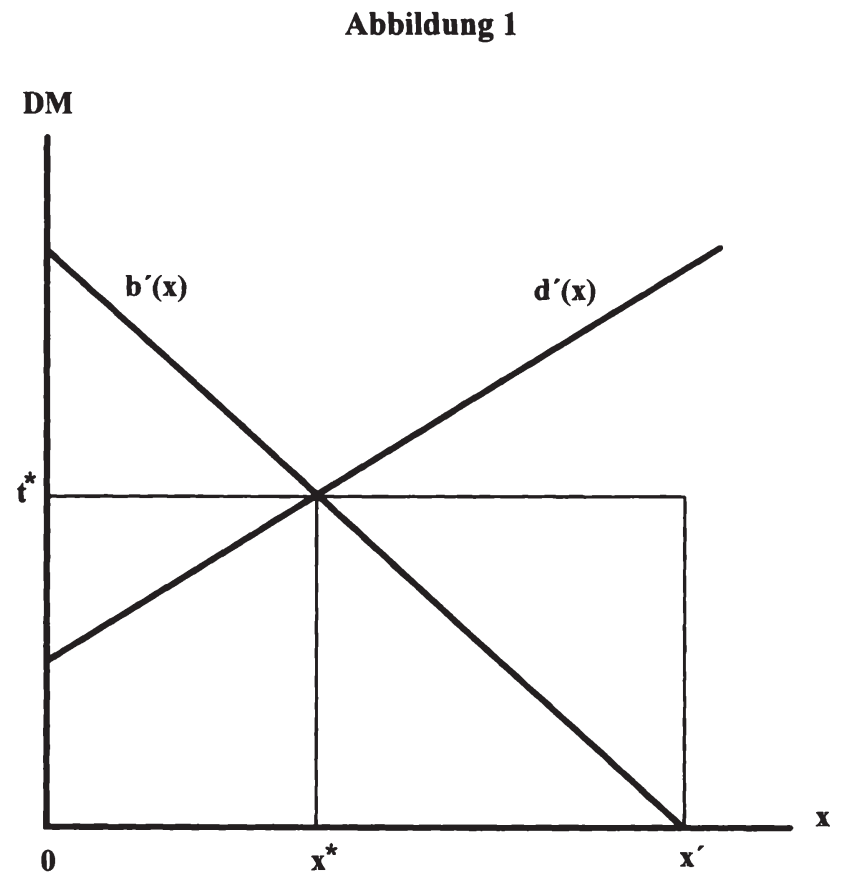

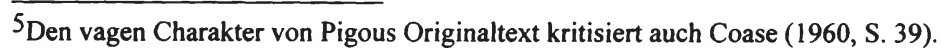


Abbildung 1 beschreibt ein einfaches Externalitätenmodell mit zwei Individuen, in dem das eine (der Verursacher oder Schädiger) einen negativen externen Effekt auf das andere (den Geschädigten) ausübt. Das Aktivitätsniveau des Schädigers (z.B. die Produktionsmenge eines bestimmten Gutes oder die Einsatzmenge eines bestimmten Produktionsfaktors) sei mit $x$ bezeichnet. Die Kurve $b^{\prime}(x)$ stehe für den Zusatzgewinn, den der Verursacher durch die Erhöhung des Aktivitätsniveaus um eine marginale Einheit erzielen kann. Die Kurve d'(x) stelle den zusätzlichen Schaden dar, der dem Geschädigten durch eine marginale Erhöhung von $\mathrm{x}$ zugefügt wird. Der gesamte Gewinn $b(x)$ bzw. der gesamte Schaden $d(x)$ entspricht damit der Fläche unterhalb der jeweiligen Kurve.

In Abwesenheit jeglicher staatlicher Regulierungsmaßnahmen würde der Verursacher das Aktivitätsniveau x' wählen, bei dem sein persönlicher Gewinn maximal ist. Bei $x^{\prime}$ ist aber der Grenzschaden des Geschädigten größer als der Grenzgewinn des Schädigers, so daß aus gesamtwirtschaftlicher Sicht eine Einschränkung der Aktivität sinnvoll wäre. Das pareto-optimale Aktivitätsniveau läge bei $\mathrm{x}^{*}$, wo der Grenzschaden mit dem Grenzgewinn übereinstimmt, denn dort ist der gesamtwirtschaftliche Wohlfahrtsgewinn $b(x)-d(x)$, der durch die Ausübung der Aktivität erzielt werden kann, maximal.

Um die Durchsetzung von $x^{*}$ zu erzwingen, kann der Staat nun beim Verursacher eine Steuer in Höhe von $\mathrm{t}^{*}=\mathrm{d}^{\prime}\left(\mathrm{x}^{*}\right)=\mathrm{b}^{\prime}\left(\mathrm{x}^{*}\right)$ pro Einheit $\mathrm{x}$ erheben. Dadurch entstehen diesem zusätzliche Kosten $t^{*} x$, und der erzielbare Nettogewinn beträgt nur noch $b(x)-t * x$. Das individuelle Gewinnmaximum des Verursachers liegt jetzt bei demjenigen Aktivitätsniveau, bei dem die Bedingung $b^{\prime}(x)=t^{*}$ erfüllt ist. Dies ist aber per Definition von $\mathrm{t}^{*}$ genau bei $\mathrm{x}^{*}$ der Fall. Der externe Effekt kann also vollständig internalisiert werden, wenn der Staat eine Mengensteuer mit dem "richtigen" Steuersatz erhebt. Dieser Steuersatz muß exakt dem verursachten Grenzschaden im Pareto-Optimum entsprechen.

Als Alternative zu dieser Pigou-Steuer bietet sich auch die Gewährung einer Subvention an. In diesem Fall gestattet man dem Schädiger die uneingeschränkte Ausübung seiner Aktivität, zahlt ihm aber für jede Einheit, um die er sein ursprünglich optimales Aktivitätsniveau $x^{\prime}$ unterschreitet, einen Subventionsbetrag in Höhe von $t^{*}$. Sein Gewinn ist nun durch $b(x)+t^{*}\left(x^{\prime}-x\right)$ gegeben und man sieht sofort, $\mathrm{da} ß$ die Bedingung für ein Gewinnmaximum mit der im Steuerfall übereinstimmt. Wenn man im Rahmen dieses einfachen Modells bleibt und insbesondere nicht danach fragt, was mit den eingenommenen Steuern geschieht bzw. woher die Mittel 
für die Subventionen stammen, dann sind die allokativen Wirkungen von Steuern und Subventionen identisch.

Neben Steuern und Subventionen sind als weitere staatliche Lenkungsinstrumente die v.a. im Rahmen der Umweltökonomie diskutierten Auflagen- und Zertifikatslösungen zu nennen. Auf diese beiden Maßnahmen, die von der Grundidee her ebenfalls in die Pigou-Tradition einzuordnen sind, wird aber hier zunächst nicht näher eingegangen.

\subsection{Der "Property-Rights"-Ansatz von Coase}

Vierzig Jahre nach dem Erscheinen von "The Economics of Welfare" übte Ronald Coase (1960) in einem bahnbrechenden Artikel recht harsche Kritik an Pigous Vorschlag zur Bewältigung von Externalitätsproblemen. Seiner Meinung nach sei der Ruf nach Staatseingriffen zu voreilig, weil der Pigou-Ansatz nicht alle Möglichkeiten einer erfolgreichen Internalisierung durch den freien Markt berücksichtige. Coases Gedanken, die diese Ansicht begründen, finden sich in den ersten Kapiteln seines Aufsatzes, in denen er anhand eines Beispiels erläutert, daß Ineffizienzen, die durch externe Effekte verursacht werden, prinzipiell auch durch private Verhandlungen zwischen den involvierten Individuen beseitigt werden können. Die in späteren Jahren als "Coase-Theorem" bezeichnete Kernaussage dieser relativ langwierigen Argumentation wurde von Turvey (1963) unter Verwendung der graphischen Darstellung, die schon Abbildung 1 zugrundegelegt wurde, auf den Punkt gebracht. (Siehe Abbildung 2).

Die Ausgangslage sei dadurch gekennzeichnet, daß der Staat keinerlei Maßnahmen ergreift, um die Ausübung des negativen externen Effekts zu begrenzen. Es werden also weder Steuern erhoben noch ist der Verursacher gesetzlich verpflichtet, für die angerichteten Schäden aufzukommen. Die Behauptung ist nun, daß die Individuen in dieser Ausgangssituation das effiziente Aktivitätsniveau selbständig und ohne Zwang durch eine zentrale Instanz realisieren werden. Diese Behauptung wird folgendermaßen begründet:

Das Aktivitätsniveau $x^{\prime}$ wird nicht dauerhaft bestehen bleiben, weil aufgrund der Divergenz zwischen Grenzgewinn und Grenzkosten durch eine Reduzierung ein zusätzlicher Wohlfahrtsgewinn möglich ist. Dieser potentielle Wohlfahrtsgewinn stellt für den Verursacher und den Geschädigten einen Anreiz dar, miteinander in 


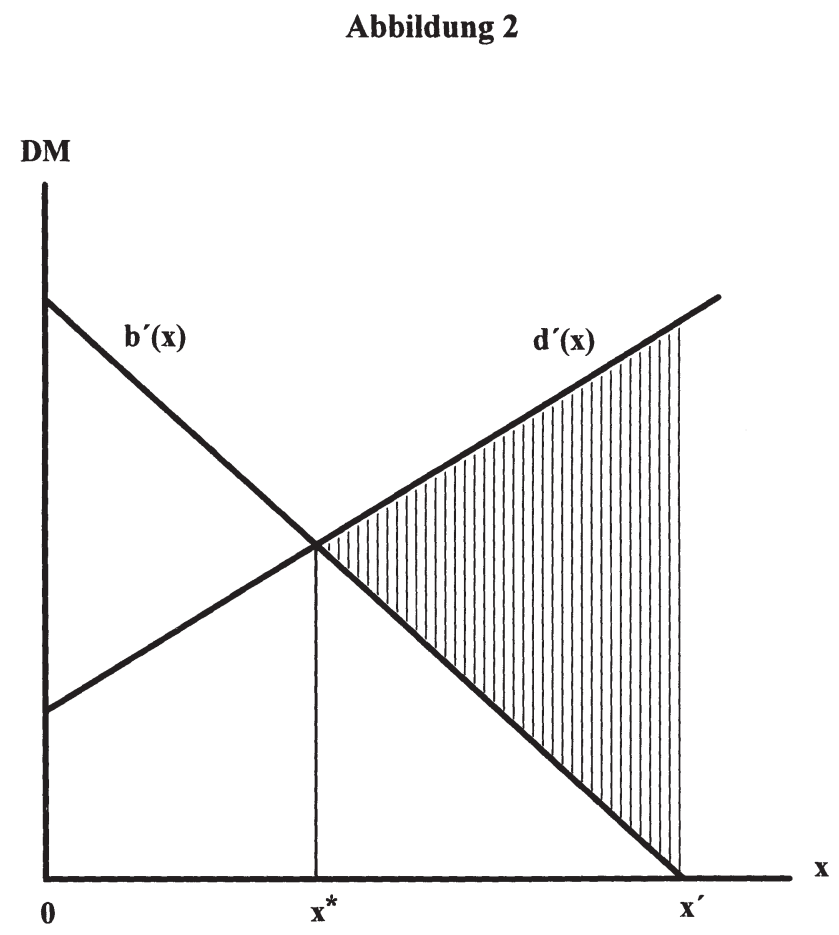

Verhandlungen über eine Einschränkung der Aktivität und die Aufteilung des damit verbundenen Gewinns einzutreten. Da rationale Individuen einen solchen Verhandlungsspielraum nützen werden und dieser erst erschöpft ist, wenn Grenzgewinn und Grenzkosten übereinstimmen, wird sich zwangsläufig das effiziente Aktivitätsniveau einstellen.

So ist es beispielsweise denkbar, daß der Geschädigte dem Schädiger für die Einschränkung seiner Aktivität eine Kompensation in Höhe der damit verbundenen Gewinneinbußen anbietet. Da sich der Schädiger durch dieses Angebot im Vergleich zur Ausgangssituation nicht verschlechtert, hat er keinen Grund, es abzulehnen. Für den Geschädigten lohnt sich eine Reduzierung des Aktivitätsniveaus, solange sein Grenzschaden höher ist als die marginale Kompensationszahlung, die dem Grenzgewinn des Schädigers entspricht. Das für ihn individuell optimale Aktivitätsniveau ist demzufolge identisch mit dem Pareto-Optimum $x^{*}$. Die Entschädigung, die er an den Verursacher zu zahlen hat, beträgt $b\left(x^{\prime}\right)-b\left(x^{*}\right)=\int_{x^{\prime}}^{x^{\prime}} \cdot b^{\prime}(x) d x$. 
Sein eigener Bruttonutzenzuwachs kann durch die vermiedene Schadensdifferenz $d\left(x^{\prime}\right)-d\left(x^{*}\right)=\int_{x^{*}}^{x^{\prime}} \cdot d^{\prime}(x) d x$ quantifiziert werden, so daß der Nettonutzenzuwachs $d\left(x^{\prime}\right)-d\left(x^{*}\right)-\left[b\left(x^{\prime}\right)-b\left(x^{*}\right)\right]=\int_{x^{\prime}}^{x^{\prime}} d^{\prime}(x)-b^{\prime}(x) d x$ beträgt. Dieser entspricht in Abbildung 2 der schraffierten Dreiecksfläche und ist offensichtlich gleich dem gesamtwirtschaftlichen Wohlfahrtsgewinn, der beim Übergang von $\mathrm{x}^{\prime}$ nach $\mathrm{x}^{*}$ erzielbar ist.

Als Alternative zu dieser Verhandlungslösung wäre es genauso denkbar, daß der Schädiger dem Geschädigten die Reduktion von $x^{\prime}$ auf $x^{*}$ gegen eine Kompensation in Höhe von $d\left(x^{\prime}\right)-d\left(x^{*}\right)=\int_{x^{\prime}}^{x^{\prime}} \cdot d^{\prime}(x) d x$ von sich aus anbietet. Die Differenz zwischen diesem Betrag, der der maximalen Zahlungsbereitschaft des Geschädigten für die Senkung des Aktivitätsniveaus von $x^{\prime}$ auf $x^{*}$ entspricht, und den Gewinneinbußen $b\left(x^{\prime}\right)-b\left(x^{*}\right)=\int_{x^{\prime}}^{x^{\prime}} \cdot b^{\prime}(x) d x$ ergibt den Nettogewinn, den der Schädiger durch dieses Arrangement erzielt. Dieser Nettogewinn ist wieder gleich dem gesamtwirtschaftlichen Überschuß.

Neben diesen beiden Extremfällen, in denen sich jeweils ein Verhandlungspartner den gesamten Wohlfahrtsgewinn aneignen kann, kommen als Verhandlungsergebnisse auch alle Zwischenlösungen in Frage, die eine Aufteilung des Überschusses vorsehen. Wenn sich die Verhandlungspartner auf einen bestimmten Verteilungsschlüssel geeinigt haben, dann ist klar, daß beide für das Aktivitätsniveau $\mathrm{x}^{*}$ plädieren werden, da andernfalls der zu verteilende "Kuchen" nicht maximal wäre.

Individuelle Nutzenmaximierung führt also in diesen Beispielen zur Durchsetzung des effizienten Aktivitätsniveaus $\mathrm{x}^{*}$ auch ohne Staatseingriffe. Alles was der Staat in dieser Situation zu tun habe, so wird weiter argumentiert, sei die Garantie einer Eigentumsordnung, in der festgelegt werden solle, ob der Schädiger, wie in den obigen Beispielen, a priori das Recht auf eine uneingeschränkte Ausübung seiner Aktivität haben soll (Laisser-Faire-Prinzip) oder ob dem Geschädigten ein Vetorecht gegen eine Beeinträchtigung eingeräumt werden soll (Verursacherprinzip). Die Tatsache, daß im ersten Fall der Geschädigte für eine Einschränkung der Produktion zu zahlen habe, im zweiten Fall dagegen der Schädiger sich die Erlaubnis zur Aufnahme der Produktion erst erkaufen müsse, habe keinen Einfluß auf die Effizienzeigenschaften der Verhandlungslösung. 6 Wenn also die privaten Wirt-

\footnotetext{
${ }^{6}$ Vgl. Coase (1960, S. 8). Auf eine Beschreibung möglicher Verhandlungslösungen im Falle des Verursacherprinzips wird hier zunächst verzichtet. Dieses Szenario wird in den folgenden beiden Kapiteln ausführlich behandelt. Die Tatsache, daß sich bei allen Varianten das gleiche pareto-op-
} 
schaftssubjekte bei klaren Eigentumsverhältnissen selbst für eine vollständige Internalisierung sorgen können, seien weitergehende Interventionen durch eine Zentralinstanz bestenfalls überflüssig, wenn nicht gar schädlich. Diese Ansicht kommt bildhaft in einem Zitat von Worcester (1972, S. 5) zum Ausdruck, in dem Staatseingriffe in dieser Situation als "drugs for a patient who is not ill" bezeichnet werden.

\subsection{Hauptargumente der Pigou-Coase-Kontroverse}

Wenn man die Vorschläge von Pigou und Coase auf dieser stark vereinfachenden Ebene vergleicht, dann stellt sich die Frage, worin nun eigentlich die Unterschiede zwischen beiden Ansätzen zu suchen sind. Bei bisheriger Sicht der Dinge könnte man jedenfalls zu der Ansicht gelangen, daß beide Konzepte zumindest unter allokativen Gesichtspunkten gleichwertig und Diskussionen für und wider Staatseingriffe im Grunde genommen überflüssig sind. Dies ist zumindest der Eindruck, den viele Lehrbuchdarstellungen dem Leser, der sich zum erstenmal mit der Thematik externer Effekte befaßt, vermitteln. Das liegt daran, daß diese Darstellungen von Rahmenbedingungen ausgehen, die jeweils die Idealvoraussetzungen für die Gültigkeit beider Theorien schaffen. Unterschiede ergeben sich erst, wenn man über diesen eng gesteckten Rahmen hinausgeht.

\subsubsection{Einwände gegen den Pigou-Ansatz}

Bereits Coase selbst wies, was die Grenzen bzw. die Schädlichkeit einer staatlichen Regulierung betrifft, auf die Möglichkeit eines sog. "Staatsversagens" hin.? So sei von einer fehlbaren Bürokratie, die anstatt Wettbewerbszwängen eher politischem Druck ausgesetzt sei, nicht zu erwarten, daß sie im Vergleich zu einer dezentralen Problemlösung höhere Effizienzgewinne realisieren könne. Für dieses Argument spricht beispielsweise, daß von vielen staatlichen Maßnahmen, die

timale Aktivitätsniveau einstellt, ist in diesem einfachen Modell durch das Fehlen von Einkommenseffekten bedingt. In einem Modell, in dem Einkommenseffekte eine Rolle spielen, ist das Verhandlungsergebnis zwar effizient, aber nicht mehr unabhängig von der Haftungsregel. Der Teil des Coase-Theorems, der als "Invarianzthese" bezeichnet wird, gilt dann also nicht. (Vgl. Endres (1977, S. 639 f.)).

${ }^{7}$ Vgl. Coase (1960, S. 18). 
prinzipiell zur Steigerung der gesamtwirtschaftlichen Wohlfahrt, gemessen etwa am Zuwachs des Sozialprodukts, führen, nicht alle Individuen der Gesellschaft profitieren, sondern daß es meist auch "Verlierer" gibt, die sich schlechter stellen. Wenn in solchen Situationen die negativen Verteilungswirkungen nicht durch entsprechende Kompensationsmaßnahmen gemildert werden können, so ist es möglich, daß die Durchführung der jeweiligen Maßnahme bei einem Großteil der Bevölkerung auf Ablehnung stößt. Berücksichtigt man, daß die Regierung eines demokratischen Staates ein Interesse an ihrer Wiederwahl hat, dann erscheint es aus ihrer Sicht u.U. geboten, auf die Verwirklichung der Maßnahme zu verzichten. Das Effizienzziel tritt dann gegenüber wahlstrategischen Überlegungen in den Hintergrund.

Ein weiterer Punkt, der im Zusammenhang mit Staatsversagen häufig angeführt wird, läuft darauf hinaus, daß sich der Staat im Falle einer Regulierung externer Effekte mittels einer Pigou-Steuer eher von dem Motiv der Erzielung eines möglichst hohen Steueraufkommens leiten lassen könnte. Da eine Anhebung der Steuer die privatwirtschaftlichen Kosten der Externalität bei deren Verursachern erhöht, wird mit dem Aktivitätsniveau die Bemessungsgrundlage und möglicherweise auch das Steueraufkommen kleiner. Dieser sog. "Laffer-Effekt" spielt z.B. in der Diskussion um das Ausmaß der Erhöhung der Mineralölsteuer eine gewisse Rolle. Verschiedene Umweltverbände sind der Ansicht, daß die Mineralölsteuer unter ökologischen Gesichtspunkten viel drastischer erhöht werden sollte als tatsächlich geschehen. Sie unterstellen der Regierung, sie wolle den Mineralöverbrauch nicht wirklich einschränken sondern die in den gegenwärtigen Preisbereichen offensichtlich relativ unelastische Nachfrage weitgehend konstant halten und damit das Steueraufkommen erhöhen. Das umweltpolitische Motiv sei nur ein Deckmantel für dieses Ziel.

Ein weiterer Einwand gegen die Erhebung einer Pigou-Steuer geht zurück auf eine Arbeit von Buchanan und Stubblebine (1962). Dieser Einwand wurde ebenfalls von Turvey (1963) im Rahmen der oben eingeführten graphischen Darstellung verdeutlicht (siehe Abbildung 3): Um mit Hilfe einer Pigou-Steuer die vollständige Internalisierung des externen Effekts zu erreichen, müßte der Steuerbetrag $t^{*}$, den der Schädiger pro Mengeneinheit zu zahlen hat, mit dem Grenzschaden im Pareto-Optimum, also mit $\mathrm{d}^{\prime}\left(\mathrm{x}^{*}\right)$, übereinstimmen. Dieser Steuerbetrag entspricht in der Graphik der Strecke Px* . Der Grenzgewinn des Schädigers nach Steuern ist dann durch $b^{\prime}(x)$ - $t^{*}$ gegeben. Da jetzt aber an der Stelle $x^{*}$ bei einer weiteren Reduktion des Externalitätsniveaus die marginale Kosteneinsparung beim Geschädigten größer ist 


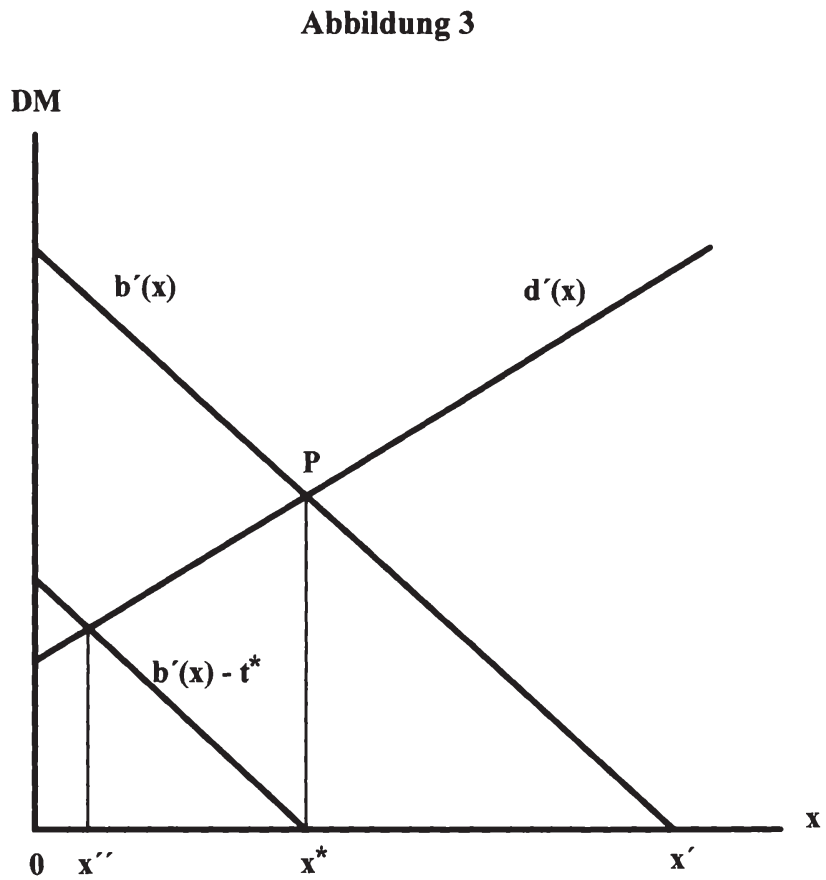

als die marginale Gewinneinbuße des Schädigers, besteht immer noch ein gewisser Verhandlungsspielraum: Der Geschädigte kann seine Position weiter verbessern, indem er dem Schädiger für eine zusätzliche Senkung des Aktivitätsniveaus auf x" eine Kompensation in Höhe von $\int_{x^{\prime \prime}}^{x^{*}} b^{\prime}(x)-t^{*} d x$ anbietet. Der Schädiger hätte keine Veranlassung, dieses Angebot abzulehnen, da es ihn nicht schlechter stellt. Bei der dann erreichten Verhandlungslösung $x^{\prime \prime}$ ist aber der gesamtwirtschaftlich relevante marginale Bruttogewinn des Schädigers größer als der externe Grenzschaden, so $\mathrm{da} B$ ein ineffizient niedriges Aktivitätsniveau resultiert. Ohne die Erhebung der Pigou-Steuer hätten Verhandlungen zu einem pareto-optimalen Ergebnis geführt. Die Steuer wird also in dieser Situation selbst zur Ursache der Fehlallokation. 


\subsubsection{Einwände gegen den Property-Rights-Ansatz}

Diejenigen Ökonomen, die dem Staat bei der Internalisierung externer Effekte mehr zutrauten als dem freien Markt, versuchten weniger, die oben genannte Kritik unmittelbar zurückzuweisen, als ihrerseits die Position der Coase-Anhänger mit den unterschiedlichsten Argumenten aufzuweichen.

Zur Begründung der eingeschränkten Tauglichkeit des von Coase propagierten dezentralen Lösungsansatzes wird häufig das sogenannte "Transaktionskosten-Argument" angeführt: Wird über das Ausmaß eines externen Effekts verhandelt, so könne dies Kosten verursachen, die beispielsweise auf die Organisation des Verhandlungsprozesses selbst, auf Probleme mit der Identifikation der Verhandlungspartner oder auf Schwierigkeiten bei der Überwachung der Einhaltung der Verträge zurückzuführen seien. Übersteigen diese Kosten die möglichen Wohlfahrtsgewinne einer Internalisierung, so unterbleibe eine aus gesamtwirtschaftlicher Perspektive prinzipiell erwünschte Vereinbarung. Je größer die Anzahl der involvierten Individuen, desto höher dürften tendenziell diese Transaktionskosten sein. Coase selbst hat diesen Einwand in seiner Arbeit bereits vorweggenommen, wies jedoch ausdrücklich darauf hin, daß staatliche Maßnahmen ebenfalls mit Transaktionskosten verbunden seien. ${ }^{8}$ Die Tatsache, daß die Marktlösung in dieser Hinsicht nicht vollkommen sei, erlaube deshalb noch nicht die Schlußfolgerung, eine zentrale Regulierung müsse zwangsläufig überlegen sein.

Als weiteres Standardargument gegen die Brauchbarkeit des Coase-Ansatzes ist das sogenannte "Free-Rider-Problem" zu nennen: Wenn beispielsweise mehrere Individuen von einem negativen externen Effekt beeinträchtigt werden und einen Schädiger prinzipiell durch Ausgleichszahlungen zu einer Einschränkung seiner Aktivität bewegen könnten, so hat eine solche Einschränkung den Charakter eines öffentlichen Gutes: Jeder Geschädigte profitiert von der Reduktion in gleicher Weise (Nicht-Rivalität), auch wenn er sich selbst nicht an den Zahlungen beteiligt (NichtAusschließbarkeit). Im Extremfall (bei sehr vielen Geschädigten) hat die Verweigerung des Beitrags durch ein einzelnes Individuum aus dessen Sicht einen vernachlässigbaren Effekt auf das vereinbarte Aktivitätsniveau, so daß der Einzelne seine Nutzenposition verbessern kann, wenn alle anderen ihren Beitrag leisten, er selbst aber nicht. Wenn alle Geschädigten diese "Schwarzfahrerposition" einnehmen wol-

\footnotetext{
${ }^{8}$ Siehe Coase (1960, S. 15 f. u. S. 19).
} 
len, verweigert letztendlich jeder seinen Beitrag und es kommt gar nicht erst zum Vertragsabschluß. ${ }^{9}$

Ein dritter Einwand wird offenbar, wenn man sich nochmals die eingangs erwähnten Beispiele für mögliche Verhandlungslösungen vergegenwärtigt. In diesen Beispielen wurde das Problem der Aufteilung des Wohlfahrtsgewinns als gelöst betrachtet, sei es in der Form, daß sich einer der Verhandlungspartner den gesamten Internalisierungsgewinn aneignen konnte, oder da $\beta$ beide sich auf einen Verteilungsschlüssel einigen konnten. Tatsächlich ist jedoch zu befürchten, daß der Interessengegensatz bei der Aufteilung des Wohlfahrtsgewinns zu Ineffizienzen führt, weil die Durchsetzung der pareto-optimalen Allokation erst nach einer diesbezüglichen Einigung erfolgen kann. Solange jedoch keine Übereinstimmung zwischen den verhandelnden Parteien besteht, drohen Wohlfahrtsverluste durch eine unnötig lange Aufrechterhaltung des ineffizienten Zustandes. Darüber hinaus können sich u.U. während der Verhandlungen über den Verteilungsschlüssel die allgemeinen Rahmenbedingungen (z.B. Technologien, Präferenzen) ändern, was die Einigung noch weiter hinauszögern würde. ${ }^{10}$ Über derartige Hindernisse könnte sich eine Zentralinstanz per Dekret leicht hinwegsetzen.

Als letzter traditioneller Kritikpunkt am Coase-Theorem sei noch ein Argument angesprochen, das auf die Möglichkeit des sog. "Rent-Seekings" bei Gültigkeit des Laisser-Faire-Prinzips abzielt und der von Coase vertretenen Auffassung widerspricht, es sei für eine erfolgreiche Internalisierung unerheblich, ob dem Schädiger oder dem Geschädigten das Eigentumsrecht zugesprochen werde. Dieses Argument geht auf Mumey (1971) zurück und wurde ebenfalls anhand der von Turvey entwikkelten Graphik präsentiert. (Siehe Abbildung 4).

Die Ausgangssituation sei dadurch gekennzeichnet, da $B$ ein Unternehmen die Wahl habe, eines von zwei Projekten, A oder B, durchzuführen. Beide Projekte bringen den gleichen Gewinn, Projekt B verursache aber im Gegensatz zu Projekt A einen externen Schaden. Der Grenzgewinn des Verursachers sei wieder mit $b^{\prime}(x)$, der Grenzschaden des Betroffenen bei Durchführung von Projekt B mit d'(x) bezeichnet. Der rechtliche Rahmen, innerhalb dessen Verhandlungen zwischen Schädiger und Geschädigtem stattfinden können, sei durch das Laisser-Faire-Prinzip definiert; der Geschädigte müßte also den Schädiger für eine Reduktion von $\mathrm{x}$

\footnotetext{
${ }^{9}$ Vgl. z.B. auch Calabresi (1967), Mishan (1971, S. 21 ff.), Endres (1977, S. 646) oder Weimann $(1990$, S.45 f.).

10Vgl. Endres (1977, S. 642).
} 


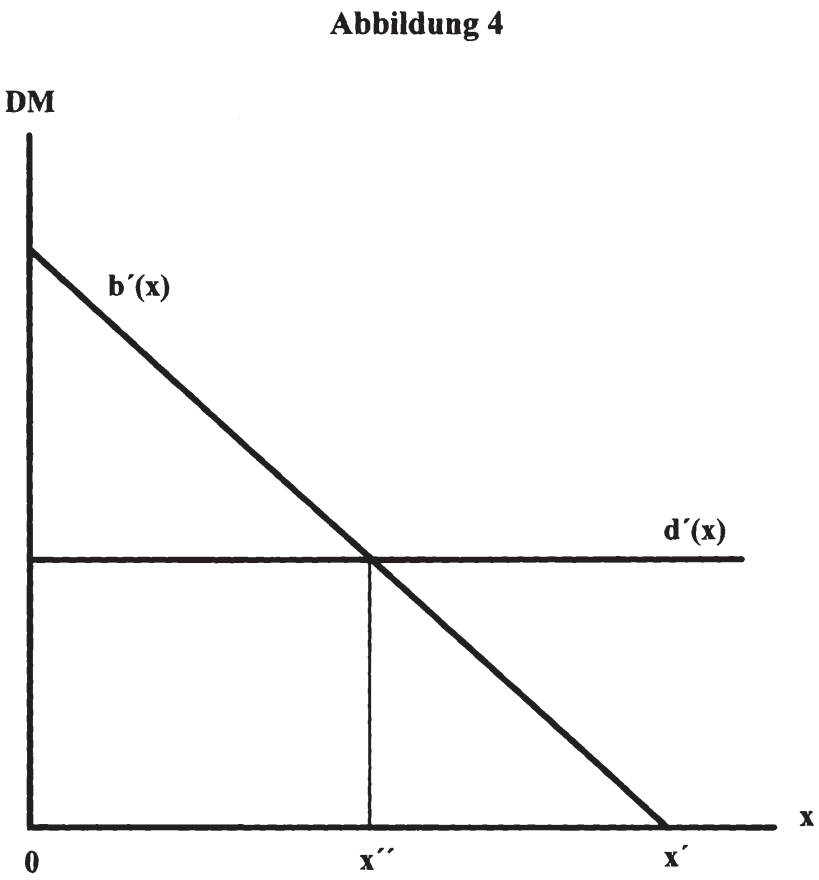

kompensieren. Es wäre in der geschilderten Situation natürlich effizient, Projekt A durchzufuihren und auf dem Niveau $x^{\prime}$ zu produzieren. Für den Schädiger macht es allerdings zunächst keinen Unterschied, für welches Projekt er sich entscheidet: In beiden Fällen beträgt sein Gewinn $b\left(x^{\prime}\right)=\int_{0}^{x^{\prime}} b^{\prime}(x) d x$, nur die Zusammensetzung hängt davon ab, welches Projekt er verwirklicht: Bei Durchführung von Projekt $\mathrm{A}$ resultiert der Gewinn ausschließlich aus der laufenden Produktion, bei Durchführung von Projekt B kann der Schädiger davon ausgehen, daß der Geschädigte ihm freiwillig eine Kompensationszahlung in Höhe von $b\left(x^{\prime}\right)-b\left(x^{\prime \prime}\right)=\int_{x^{\prime \prime}}^{x^{\prime}} b^{\prime}(x) d x$ für eine Einschränkung des Outputs auf $x^{\prime \prime}$ anbieten wird. Der restliche Gewinn stammt dann ebenfalls aus der laufenden Produktion.

Dem Geschädigten wäre dagegen eine Entscheidung zugunsten von Projekt A lieber, da er in diesem Fall keine Kosten zu tragen hätte, während er bei Durchführung von Projekt $B$ an den Schädiger $\int_{x^{\prime \prime}}^{x^{\prime}} b^{\prime}(x) d x$ zahlen und außerdem einen Schaden in Höhe von $d\left(x^{\prime \prime}\right)=\int_{0}^{x^{\prime \prime}} d^{\prime}(x) d x$ tolerieren müßte. Diesen Umstand könnte sich 
nun der Schädiger zunutze machen und dem Geschädigten androhen, er werde Projekt $B$ realisieren, wenn er nicht mindestens eine Zahlung in Höhe von $\int_{0}^{x^{\prime \prime}} d^{\prime}(x) d x$ $+\int_{x^{\prime \prime}}^{x^{\prime}} b^{\prime}(x) d x$ erhalten würde. Diese Drohung wäre glaubhaft, da der Schädiger im Falle einer Ablehnung dieser Forderung indifferent wäre zwischen der Durchführung von Projekt A und Projekt B. Der Geschädigte könnte sich seinerseits durch eine Verweigerung dieser Zahlung nicht besserstellen, so daß die endgültige Verhandlungslösung wie folgt charakterisiert werden kann: Der Schädiger wählt Projekt $A$, produziert die Menge $x^{\prime}$ und läßt sich für den Verzicht auf die Durchführung von Projekt B eine zusätzliche Rente in Höhe von $\int_{0}^{x^{\prime \prime}} d^{\prime}(x) d x+\int_{x^{\prime \prime}}^{x^{\prime}} b^{\prime}(x) d x$ zahlen.

Dieses Verhandlungsergebnis scheint auf den ersten Blick kein allokatives Problem aufzuwerfen, denn die Wahl von Projekt A und Produktionsmenge $x^{\prime}$ ist effizient. Mumey bringt dagegen jedoch einen Einwand vor, der nicht unmittelbar innerhalb des gewählten Modellrahmens erkennbar ist: Man müsse berücksichtigen, daß die Möglichkeit der Erpressung solcher Zusatzrenten Anreize zur Aufwendung von Ressourcen für die Entwicklung von Drohpotentialen schaffe. Man könnte sich beispielsweise vorstellen, daß der Schädiger erst Mittel in die Entwicklung von Plänen zur Durchführung von Projekt $B$ investieren muß, um die Glaubwürdigkeit seiner Drohung unter Beweis zu stellen. Wenn diese Investitionsausgaben kleiner sind als die erzielbare Zusatzrente, lohnt sich die Erpressung für den Schädiger. Ein Ressourcenverzehr für derartige Zwecke ist natürlich aus gesamtwirtschaftlicher Sicht reine Verschwendung.

Die Gefahr der Entwicklung von Drohpotentialen der beschriebenen Art besteht nur bei Zugrundelegung des Laisser-Faire-Prinzips: Da der Schädiger im Rahmen dieser Rechtsordnung bei der Ausübung seiner Aktivität keinen Beschränkungen unterliegt, kann er für den Fall, daß der Geschädigte nicht auf seine Forderungen eingeht, jede noch so harte Drohung, sofern sie nur glaubhaft ist, wahr machen, ohne rechtliche Konsequenzen fürchten zu müssen. Dadurch ist es ihm möglich, den Geschädigten bewußt in eine ungünstige Verhandlungsposition zu drängen und höhere Kompensationszahlungen zu erpressen. Gilt dagegen das Verursacherprinzip, dann ist die Nutzenposition, die der Schädiger im Falle einer Nichteinigung erreicht, von vornherein nach unten beschränkt. Schlimmstenfalls muß er auf die Ausübung seiner Aktivität und die damit verbundenen Gewinne verzichten. Der Geschädigte hat aber keine Möglichkeit, die Verhandlungsposition des Schädigers zu schwächen, so daß für ihn kein Anreiz besteht, nach Möglichkeiten zum Aufbau von Drohpoten- 
tialen zu suchen und dafür Ressourcen zu verschwenden. ${ }^{11}$ Aus diesen Überlegungen kann gefolgert werden, daß es, anders als von Coase behauptet, sehr wohl von der Verteilung der Eigentumsrechte abhängen kann, ob die erreichte Verhandlungslösung effizient ist oder nicht.

\subsection{Unvollständige Information: Ein Problem in beiden Ansätzen}

Wenn man an dieser Stelle versucht, anhand der oben geschilderten Kontroverse eine Aussage darüber zu treffen, ob eine zentrale Regulierung externer Effekte einer dezentralen "Marktlösung" vorzuziehen ist oder nicht, wird man wohl in Schwierigkeiten geraten. Bei objektiver Betrachtung wird man zugeben müssen, daß jedem einzelnen der bisher angeführten Argumente eine gewisse Berechtigung nicht abzusprechen ist. Was bringt aber diese Erkenntnis im Hinblick auf die Beantwortung der Frage nach der geeigneten Institution zur Lösung des Externalitätenproblems? Wenn man in der eingangs eingeführten einfachen Modellwelt zu dem Schluß kam, daß sowohl durch Staatseingriffe als auch durch private Verhandlungen eine erfolgreiche Internalisierung möglich ist, so muß man nun nach genauerer Betrachtung erkennen, da $\beta$ unter etwas realistischeren Rahmenbedingungen weder das eine noch das andere Konzept in jeder Hinsicht befriedigend abschneidet. Ein Urteil darüber, welcher Lösungsvorschlag die gravierenderen Schwächen aufweist, ist aber nicht möglich, weil sich die Argumente der traditionellen Diskussion auf unterschiedliche Modellwelten beziehen, die nicht ohne weiteres miteinander vergleichbar sind. Da sowohl der Pigou-Ansatz als auch der Property-Rights-Ansatz aus den verschiedensten Gründen keine vollkommen effizienten Allokationen erwarten läßt, ist eine Gegenüberstellung der beiden Konzepte nur sinnvoll, wenn man den jeweiligen Grad ihrer Ineffizienz messen und vergleichen kann. Ein solcher Effizienzvergleich ist aber nur in einem einheitlichen Modellrahmen möglich.

Damit sind wir bei der Zielsetzung dieser Arbeit angelangt, die darin besteht, einen derartigen Vergleich durchzuführen. Dieses Vorhaben kann nur dann einen Sinn haben, wenn man ein Modell wählt, in dem die Idealvoraussetzungen für ein perfektes Funktionieren beider Lösungsansätze nicht erfüllt sind. Zu diesen Idealvoraussetzungen gehört unter anderem die Annahme vollständiger Information über

${ }^{11}$ Vgl. auch Endres (1977, S. 644). Der Artikel gibt einen Überblick über die "alte" Pigou-CoaseKontroverse. 
die entscheidungsrelevanten Eigenschaften der beteiligten Individuen. Diese Annahme, die implizit allen bisher angeführten Argumenten zugrunde lag, wurde in der Vergangenheit weniger thematisiert, obwohl sie natürlich in höchstem Maße unrealistisch ist. Dies mag daran liegen, daß die nötigen formalen Methoden erst in neuerer Zeit im Zuge des Booms der Informationsökonomie zur Verfügung gestellt wurden. Die Fortschritte auf diesem Teilgebiet der ökonomischen Theorie führten aber dann zu einer Wiederbelebung der Pigou-Coase-Kontroverse unter dem Blickwinkel unvollständiger Information. ${ }^{12}$

Was die Internalisierung externer Effekte mittels Steuern betrifft, so hat bereits Coase selbst zu bedenken gegeben, daß die Wahl des "richtigen" Steuersatzes nur dann möglich ist, wenn die regulierende Behörde sowohl die Grenzschadensfunktion des Geschädigten als auch die Grenzgewinnfunktion des Verursachers kennt. Diese Anforderung an eine Zentralinstanz wurde von ihm zu Recht als utopisch bezeichnet, und er hat diesen Einwand ansatzweise in seiner Arbeit als Kritikpunkt am Pigou-Ansatz formuliert. ${ }^{13}$

Auf die Tatsache, daß Informationsprobleme in der Dezentralisierungsdebatte eine tragende Rolle spielen, wies bereits Hayek (1945) hin. Seine Ausführungen, in denen er die Vorteilhaftigkeit einer dezentralen Ressourcenallokation über Märkte betont, lassen sich in etwa wie folgt wiedergeben: Eine Institution, die eine effiziente Allokation von Gütern und Produktionsfaktoren sichern soll, muß in erster Linie in der Lage sein, eine Fülle von Informationen darüber zu verarbeiten, in welchen Verwendungen diese Ressourcen den größten gesamtwirtschaftlichen Nutzen stiften. Über derartige Informationen (z.B. über Präferenzen und Technologien) verfügen aber in der Regel nur einzelne Wirtschaftssubjekte. Insbesondere stehen sie niemals einem zentralen Planer in ihrer Gesamtheit zur Verfügung. Auch sei die Beschaffung und Verarbeitung dieser Informationen ein aussichtsloses Unterfangen. Das ökonomische Problem könne deshalb nicht von einer Zentralinstanz gelöst werden. Der freie Markt dagegen bilde Preise als Indikatoren ökonomischer Knappheitsrelationen heraus, in denen alle für die Wirtschaftssubjekte relevanten Daten über die Ökonomie in komprimierter Form vorliegen. Die Individuen müßten also ihren Entscheidungen nur diese gegebenen Preise und die privaten Informationen über ihre eigenen Rahmenbedingungen und Zielvorstellungen zugrunde legen. Weitere Kenntnisse, etwa über Präferenzen oder Technologien anderer Wirtschafts-

12Für diese "neuere" Pigou-Coase-Kontroverse stehen in etwa die Arbeiten von Farrell (1987), Buchholz und Haslbeck (1991/1992) sowie Illing (1992).

13Vgl. Coase (1960, S. 41 f. ). 
subjekte, seien, anders als bei Anwendung eines zentralen Allokationsmechanismus, nicht erforderlich. Der Preismechanismus würde dann die Entscheidungen der Individuen so koordinieren, da $\beta$ eine effiziente Ressourcenallokation resultiere. Aus diesem Grunde sei der Markt besser dazu geeignet, das bestehende Informationsproblem zu lösen.

Diese Argumentation ist natürlich nur in Situationen korrekt, in denen vollkommener Wettbewerb herrscht, denn nur dann ist es gerechtfertigt, von gegebenen Marktpreisen auszugehen. Nun ist es aber höchst zweifelhaft, daß bei Verhandlungen, so wie Coase sie sich vorgestellt hat, Wettbewerbsbedingungen vorliegen. Gerade im überwiegend diskutierten Standardfall bilateraler Verhandlungen ist Preisnehmerverhalten mit Sicherheit keine sinnvolle Verhaltenshypothese. Es ist vielmehr davon auszugehen, daß hier Marktmacht, also die Fähigkeit der Marktteilnehmer, durch eigenes Verhalten die Tauschbedingungen zu beeinflussen, eine wichtige Rolle spielt.

Sobald Marktmacht ins Spiel kommt, taucht aber auch das Informationsproblem wieder auf. Dies leuchtet unmittelbar ein, wenn man sich verschiedene Formen von Marktmacht ansieht. So analysieren beispielsweise Sinn und Schmoltzi (1981) im Zusammenhang mit der Internalisierung externer Effekte auf dem Verhandlungswege unterschiedliche Szenarien, in denen sich, je nach Ausgestaltung der Eigentumsregelung, zumindest eine Verhandlungsseite als Monopolist bzw. Monopsonist verhält. Es gibt also in diesen Modellen Individuen, die ihre eigenen Nutzenmaximierungskalküle auf das Nachfrage- bzw. Angebotsverhalten anderer Individuen abstimmen. Da deren Verhalten aber ebenfalls auf Nutzenmaximierung basiert, die wiederum von individuellen Präferenzen abhängt, liegt hier eindeutig ein Informationsproblem vor: Wer Marktmacht ausüben will, benötigt zumindest indirekt Informationen über die Präferenzen anderer Marktteilnehmer. ${ }^{14}$

Dies äußert sich auch in den obigen Beispielen, in denen denkbare Verhandlungslösungen beschrieben wurden. Diese Verhandlungslösungen waren durch eine extreme Form von Marktmacht bestimmt, da angenommen wurde, die eine Partei könne der anderen einen Vorschlag unterbreiten, den diese nur annehmen oder ablehnen kann. Dadurch war es möglich, daß sich die offerierende Partei den gesamten Tauschgewinn aneignen konnte, während die akzeptierende Partei auf ihrem Re-

\footnotetext{
${ }^{14}$ Bei Sinn und Schmoltzi (1989) spielt dieses Informationsproblem allerdings keine Rolle. Sie analysieren die Auswirkungen unterschiedlicher Varianten der Ausgestaltung von Eigentumsrechten auf die Effizienz der Verhandlungslösung bei Vorliegen unvollkommenen Wettbewerbs unter vollständiger Information.
} 
servationsnutzenniveau gehalten wurde. Solche "Ausbeutungslösungen", die, im Gegensatz zu Standard-Monopollösungen, effizient sind, können nur durchgesetzt werden, wenn die offerierende Partei das Reservationsnutzenniveau der akzeptierenden Partei kennt. In unserem Fall wäre es also erforderlich, daß der Verursacher die Schadensfunktion des Geschädigten bzw. der Geschädigte die Gewinnfunktion des Verursachers kennt. Davon kann aber i.a. nicht ausgegangen werden.

Es ist also keineswegs so, daß Informationsprobleme bei dezentralen Internalisierungsansätzen keine Rolle spielen. Wenn man davon ausgeht, daß zwischen dem Staat und den privaten Wirtschaftssubjekten asymmetrische Information über entscheidungsrelevante Merkmale herrscht, dann muß man mit diesem Phänomen auch bei privaten Verhandlungen zwischen den Individuen rechnen. Warum sollte beispielsweise der Verursacher einer Externalität über die Schadensfunktion des Geschädigten besser informiert sein als eine zentrale Behörde? Beide müssen sich diese Information erst beschaffen, bevor sie sie nützen können. Ob sich unter diesen Umständen die dezentrale Lösung einem Staatseingriff als überlegen erweist, ist fraglich und muß erst überprüft werden. Der Versuch, einen Beitrag zur Beantwortung dieser Frage zu leisten, ist das Ziel der vorliegenden Arbeit.

\subsection{Zielsetzung und Aufbau der Arbeit}

Das Hauptanliegen besteht darin, nicht-öffentliche Information in die Modellierung sowohl privater Verhandlungen als auch staatlicher Regulierungsmaßnahmen miteinzubeziehen und zu untersuchen, welcher Lösungsansatz unter diesen einheitlichen Rahmenbedingungen im Hinblick auf eine effiziente Ressourcenallokation besser abschneidet.

Die Arbeit gliedert sich in zwei Teile. Im ersten Teil, der die Kapitel 2 und 3 umfaßt, werden die Effizienzeigenschaften privater Verhandlungen über externe Effekte bei unvollständiger Information untersucht.

In Kapitel 2 geht es um die Frage, ob in dem in der Einleitung vorgestellten einfachen Externalitätenmodell mit nur zwei Individuen die Effizienzthese des Coase-Theorems noch gilt, wenn zwischen den beiden Verhandlungspartnern asymmetrische Information über die Grenzschadens- bzw. Grenzgewinnfunktion besteht. Dabei wird zunächst ein konkreter und sehr einfacher nicht-kooperativer Verhandlungsprozeß bei symmetrischer Information modelliert. Dieser Verhandlungs- 
prozeß hat die Eigenschaft, ein effizientes Allokationsergebnis herbeizuführen. Im nächsten Schritt wird dieses Verhandlungsmodell auf eine Situation übertragen, in der dem Schädiger als offerierender Partei die Schadensfunktion des Geschädigten unbekannt ist. Der Schwerpunkt liegt dabei auf dem Bemühen, die methodischen Grundlagen des sog. "Mechanism-Design"-Ansatzes, den die Literatur zur Analyse dieses Modelltyps anbietet, ausführlicher zu erläutern und mehr mit ökonomischen Inhalten zu füllen, als dies dort getan wird. Das zentrale Resultat, daß die Verhandlungslösung bei unvollständiger Information ineffizient ist, wird ohne allzu großen formalen Aufwand hergeleitet. Den Abschluß des zweiten Kapitels bildet eine Diskussion der Modellannahmen. Es wird sich zeigen, daß die "Ineffizienzthese" über die explizit analysierte Modellversion hinaus weitgehend allgemeingültig ist.

In Kapitel 3 wird das Modell auf ein Szenario mit mehreren Geschädigten erweitert. Ziel der Analyse dieses Falls ist zu zeigen, daß sich die Effizienzeigenschaften des Verhandlungsergebnisses verschlechtern, wenn die Anzahl der Geschädigten zunimmt. Dieses Kapitel stützt sich weitgehend auf eine Arbeit von Rafael Rob (1989). Es wurde auch hier wieder versucht, die ökonomischen Inhalte im Vergleich zu den Ausführungen des Originaltexts stärker in den Vordergrund zu rücken. Um diesem Anliegen Rechnung zu tragen, erschien es angebracht, eine spezielle graphische Darstellung zu entwickeln, um Analyse und Ergebnisse transparenter erscheinen zu lassen.

Der zweite Teil der Arbeit besteht aus den Kapiteln 4 bis 6. Dort wird der Frage nachgegangen, inwieweit eine zentrale Behörde die bestehenden Informationsprobleme vergleichsweise besser bewältigen und höhere Internalisierungsgewinne erzielen kann. Dabei wird nicht zuletzt auf der Basis der Ergebnisse des dritten Kapitels davon ausgegangen, daß private Verhandlungen überhaupt nur in Situationen mit wenigen Beteiligten, insbesondere im Zwei-Personen-Fall, halbwegs akzeptable Effizienzeigenschaften aufweisen. Deshalb wird auch das bilaterale Verhandlungsmodell des zweiten Kapitels durchwegs als Vergleichsgrundlage herangezogen.

Im vierten Kapitel wird zunächst untersucht, welche Anreizprobleme bei den bekannten staatlichen Lenkungsinstrumenten (Steuern und Auflagen) auftreten, wenn die regulierende Behörde nur über beschränkte Informationen bezüglich der gesamtwirtschaftlichen Grenznutzen bzw. Grenzkosten, die mit der Externalität verbunden sind, verfügt. Das Hauptaugenmerk liegt dabei auf der Analyse strategischen Verhaltens der Individuen bei der Angabe ihrer für den Staat entscheidungsre- 
levanten Charakteristika, sowie auf den Auswirkungen dieses Verhaltens auf das Allokationsergebnis.

Kapitel 5 befaßt sich dann mit der Möglichkeit der Anwendung von Präferenzenthüllungsverfahren zur Überwindung der in Kapitel 4 beschriebenen Informationsprobleme. Es werden klassische Mechanismen, die ursprünglich für die Enthüllung von Präferenzen für öffentliche Güter entwickelt wurden, auf das Externalitätenmodell übertragen. Schwerpunktmäßig werden dabei, neben offensichtlichen Schwierigkeiten bei der praktischen Durchführung, v.a. die wichtigsten theoretischen Schwächen dieser Mechanismen überblicksartig dargestellt und im Zusammenhang diskutiert. Diese Diskussion führt zu dem Schluß, daß Präferenzenthüllungsmechanismen für eine Internalisierng externer Effekte nicht geeignet sind.

Wenn man diese Ansicht vertritt, dann stellt sich die Frage, wie alternative Lenkungsinstrumente, bei denen der Staat von vornherein auf Informationsenthüllung und damit auf Effizienz um jeden Preis verzichtet, im Vergleich zur ineffizienten privaten Verhandlungslösung unter Wohlfahrtsgesichtspunkten abschneiden. Dieser Frage wird in Kapitel 6 nachgegangen. Anhand eines Beispiels wird demonstriert, daß schon durch einfache und im Gegensatz zu den Enthüllungsmechanismen praktikable Internalisierungsmaßnahmen bessere Allokationsergebnisse erzielt werden können als durch Verhandlungen.

Zum Schluß werden die erarbeiteten Ergebnisse zusammengefaßt und im Hinblick auf die Frage, ob sich durch das Auftreten unvollständiger Information Staatseingriffe begründen lassen, bewertet. 


\section{Teil I}

\section{Die Internalisierung externer Effekte im Rahmen nicht-kooperativer Verhandlungen}

\section{Bilaterale Internalisierungsverhandlungen}

Mit seinem Aufsatz "The Problem of Social Cost", in dem er auf die Möglichkeit einer freiwilligen Internalisierung von Externalitäten durch Verhandlungen zwischen den betroffenen Individuen hinweist, hat Coase zweifellos einen bedeutenden Beitrag zur Theorie externer Effekte geleistet. Die Tragweite seiner Überlegungen geht jedoch weit über das Externalitätenproblem hinaus. Das Coase-Theorem spielt in allen Themenbereichen, die sich allgemein mit der Frage nach dem geeigneten institutionellen Rahmen zur Beseitigung bestehender Fehlallokationen auseinandersetzen, eine wichtige Rolle. Die Möglichkeit privater Verhandlungen zur Aufhebung von Allokationsstörungen muß seit Coase von jedem Ökonomen in Betracht gezogen werden, bevor er die Intervention einer Zentralinstanz fordert. Der CoaseAnsatz hat also trotz aller Kritik, die ihm vor allem von den Anhängern des "Staatsinterventionismus" entgegengebracht wird, insofern seinen unbestrittenen theoretischen Wert, als er den Zwang zur Begründung von Staatseingriffen verstärkt.

\subsection{Begründung der Notwendigkeit nicht-kooperativer Verhandlungsmodelle}

Die wichtigsten traditionellen Einwände gegen das Coase-Theorem wurden bereits in der Einleitung angesprochen. Darüber hinaus weist es aber noch eine weitere gravierende Schwachstelle auf. Diese wird unmittelbar deutlich, wenn man danach fragt, wie denn die von Coase vorgeschlagenen Verhandlungen zwischen dem Betroffenen und dem Verursacher einer Externalität konkret aussehen sollen. Diese Frage, die bei der Begründung des Coase-Theorems vollständig vernachlässigt wurde, erweist sich bei genauerer Betrachtung als überaus wichtig. Die Tatsache, daß bei Vorliegen einer Externalität ein Verhandlungsspielraum zwischen Ver- 
ursacher und Geschädigtem existiert, muß ja nicht zwangsläufig bedeuten, daß dieser Spielraum auch vollständig ausgenützt wird. Ob private Verhandlungen wirklich $\mathrm{zu}$ gesamtwirtschaftlich erwünschten Ergebnissen führen, wird ganz entscheidend vom konkreten Verhandlungsprozeß abhängen. Die Individuen orientieren sich ja, zumindest nach neoklassischer Sichtweise, nicht primär an gesamtgesellschaftlichen Optimalitätskriterien, sondern verfolgen in erster Linie ihre Eigeninteressen. In privaten Verhandlungen wird also jeder versuchen, sich einen möglichst hohen Anteil an den prinzipiell realisierbaren Wohlfahrtsgewinnen zu sichern. Daß dabei strategisches Verhalten eine entscheidende Rolle spielt, liegt auf der Hand. Vor diesem Hintergrund erscheint es wenig sinnvoll, ähnlich wie in der kooperativen Verhandlungstheorie, Effizienz quasi axiomatisch vorauszusetzen. ${ }^{1}$ Vielmehr ist es angebracht, das Problem mit den Mitteln der nicht-kooperativen Spieltheorie zu analysieren, um die Optimalität oder auch die Suboptimalität privater Verhandlungen als echtes Resultat abzuleiten. Dazu ist aber eine explizite Modellierung des Verhandlungsprozesses erforderlich. ${ }^{2}$

Wenn nun das gewählte Modell reale Verhältnisse auch nur ansatzweise widerspiegeln soll, dann ist die Berücksichtigung unvollständiger Information über entscheidungsrelevante Daten zwischen den Verhandlungspartnern unerläßlich. Wenn jede Partei über alle wichtigen Charakteristika der anderen Partei vollständig informiert wäre, wären zeitraubende und kostenintensive Verhandlungsprozeduren mit ineffizienten Ergebnissen nur schwer vorstellbar, da unter diesen Umständen sowohl das Ausmaß des Verhandlungsspielraums als auch gewisse Akzeptanzschwellen beim Verhandlungsgegner offensichtlich wären. Die Möglichkeiten strategischen Handelns sind dadurch stark eingeschränkt. So hat beispielsweise Rubinstein (1982) gezeigt, daß die Verhandlungspartner in einem sog. "Kuchenteilungsspiel" mit alternierenden Offerten bei vollständiger Information ohne unnötige Verzögerungen eine effiziente Einigung erzielen.

In Verhandlungsspielen mit unvollständiger Information sind dagegen ernsthafte Probleme zu erwarten, da die Parteien versuchen werden, ein eventuelles Informationsgefälle $\mathrm{zu}$ ihren Gunsten auszunutzen und sich durch bewußte Verschleierung charakteristischer Merkmale Vorteile zu verschaffen. Die Berücksichtigung unvollständiger Information bei der Modellierung von Verhandlungen eröffnet

\footnotetext{
${ }^{1}$ Diese Kritik wird beispielsweise auch von Arrow (1979, S.24 ff. ) oder Samuelson (1985, S. 321) geübt.

${ }^{2}$ Vgl. dazu v.a. Schweizer (1988) oder auch Weimann (1990, S. 31 ff.).
} 
also die Möglichkeit, das bei real existierenden Verhandlungen häufig beobachtete "Taktieren" ins Spiel zu bringen.

Gegenstand dieses Kapitels ist die Untersuchung der Auswirkungen unvollständiger Information auf die Effizienzeigenschaften der Verhandlungslösung in einem bilateralen Verhandlungsmodell. Um diese Auswirkungen deutlich herausarbeiten zu können, muß zunächst als Referenzrahmen die Verhandlungslösung in dem entsprechenden Modell mit vollständiger Information beschrieben werden. Dies geschieht in Abschnitt 2.3 dieses Kapitels, nachdem in Abschnitt 2.2 das grundlegende Externalitätenmodell eingeführt wurde. In Abschnitt 2.4, dem Schwerpunkt dieses Kapitels, wird unvollständige Information ins Verhandlungsmodell miteinbezogen und untersucht, inwieweit die Effizienz der Verhandlungslösung dadurch beeinträchtigt wird. Es wird sich zeigen, da $B$ das Verhandlungsergebnis hier im Gegensatz zum Modell mit vollständiger Information ineffizient ist. Dieses Ergebnis wird in Abschnitt 2.5 anhand eines Beispiels illustriert. Da den Resultaten des Abschnitts 2.4 sehr spezielle Annahmen zugrunde liegen, wird in Abschnitt 2.6 begründet, warum diese auch unter allgemeineren Rahmenbedingungen zu erwarten sind.

\subsection{Das Grundmodell}

In diesem Abschnitt werden die elementaren Bausteine des einfachen Externalitätenmodells beschrieben, das die Grundlage für die folgenden Analysen bilden wird. Es handelt sich dabei im wesentlichen um das Modell, das bereits in der Einführung verwendet wurde. Dieses Modell muß nur noch etwas genauer spezifizert werden, damit sich später der formale Aufwand in vertretbaren Grenzen hält. AuBerdem wird eine stochastische Komponente eingebaut, die erforderlich ist, um später unvollständige Information einführen zu können. Um eine möglichst geschlossene Darstellung der behandelten Sachverhalte zu ermöglichen, soll das Grundmodell immer nur so weit modifiziert werden, wie es eine Veranschaulichung der zentralen Inhalte erfordert.

Betrachtet wird ein Unternehmen, das ein bestimmtes Gut herstellt, dessen Produktionsmenge mit $\mathrm{x}$ bezeichnet werden soll. Das Unternehmen erziele durch die Herstellung dieses Gutes einen Gewinn $b(x)$. Die Gewinnfunktion sei konkav, zweimal stetig differenzierbar und weise an der Stelle $\mathrm{x}=\mathrm{x}^{\prime}$ ein (globales) Maxi- 
mum auf. Für die Grenzgewinnfunktion gilt also $b^{\prime}\left(x^{\prime}\right)=0$, und es ist $b^{\prime \prime}(x)<0$ über den gesamten Definitionsbereich.

In unmittelbarer Umgebung der Produktionsanlagen des Unternehmens wohne ein einzelner Anlieger, der durch die Herstellung des Gutes einen Schaden erleidet. Von diesem Schaden wird angenommen, er könne in monetären Einheiten gemessen und durch die Schadensfunktion $\mathrm{d}(\mathrm{x})=\theta \mathrm{x}$ angegeben werden. ${ }^{3}$ Zur inhaltlichen Verdeutlichung des geschilderten Problems kann man sich vorstellen, daß mit der Herstellung von $\mathrm{x}$ ein Schadstoff verbunden ist, auf den nicht alle Individuen gleichermaßen empfindlich ansprechen. Je nachdem, wie "allergisch" der Anwohner auf den freigesetzten Schadstoff reagiert, ist sein erlittener Schaden unterschiedlich hoch. Der "Schädlichkeitskoeffizient" $\theta$ ist eine Zufallsvariable, die prinzipiell jeden Wert im Intervall $\mathrm{T}=\left[\theta^{-}, \theta^{+}\right]$annehmen kann. Der Anlieger ist also statistisch gesehen ein Element aus der Grundgesamtheit aller möglichen "Typen" von Geschädigten. Innerhalb dieser Grundgesamtheit sei $\theta$ gemäß der stetigen Wahrscheinlichkeitsfunktion $F(\theta)$ mit der entsprechenden Wahrscheinlichkeitsdichte $f(\theta)=F^{\prime}(\theta)$ verteilt.

Unter diesen Umständen wäre aus gesamtwirtschaftlicher Sicht für jeden Schadenstyp, der auftreten kann, ein anderes Produktionsniveau optimal. Für den Fall, da $\beta$ der Anlieger den Schadenstyp $\theta$ aufweist, liegt die effiziente Produktionsmenge, bei der die gesamtwirtschaftliche Nettowohlfahrt $\mathrm{W}(\mathrm{x})=\mathrm{b}(\mathrm{x})-\mathrm{d}(\mathrm{x})=\mathrm{b}(\mathrm{x})$ $\theta \mathrm{x}$ maximiert wird, an der Stelle, an der der marginale Gewinn des Unternehmens mit dem Grenzschaden $\theta$ des Anliegers übereinstimmt. Bezeichnet man die effiziente Produktionsmenge mit $x^{*}(\theta)$, dann gilt demnach im Optimum $b^{\prime}\left(x^{*}(\theta)\right)=\theta$. Aufgrund der Annahme eines abnehmenden Grenzgewinns ist $x^{*}(\theta)$ umso höher, je niedriger der Grenzschaden des Geschädigten ist.

Der gesamtwirtschaftliche Wohlfahrtsgewinn, der durch die Herstellung des Gutes erzielt werden kann, beträgt dann für eine gegebene Realisierung des Schadensparameters $\mathrm{W}(\theta)=\mathrm{b}\left(\mathrm{x}^{*}(\theta)\right)-\theta \mathrm{x}^{*}(\theta)$. Falls man einen Allokationsmechanismus finden könnte, der immer dazu führen würde, daß die jeweils effiziente Produktionsmenge $x^{*}(\theta)$ hergestellt würde, dann ergäbe sich ex ante, also bevor bekannt

\footnotetext{
${ }^{3}$ Die Annahme eines konstanten Grenzschadens ist nicht entscheidend für die inhaltlichen Resultate (vgl. Buchholz und Haslbeck (1991/1992) oder Illing (1992)), erleichtert jedoch die formale Analyse nach Einführung unvollständiger Information. Im übrigen liefert diese Annahme dieselbe Modellstruktur, die auch in den einschlägigen Literaturstellen überwiegend Verwendung findet (z.B. Myerson (1981), Myerson und Satterthwaite (1983) oder Rob (1989)).
} 
wird, welcher Grenzschaden $\theta \in \mathrm{T}$ beim Geschädigten wirklich vorliegt, ein erwarteter gesamtwirtschaftlicher Wohlfahrtsgewinn in Höhe von

$$
W^{*}=\int_{\theta^{-}}^{\theta^{+}}\left[b\left(x^{*}(\theta)\right)-\theta x^{*}(\theta)\right] f(\theta) d \theta
$$

\subsection{Ein einfaches Verhandlungsmodell mit vollständiger Information}

In diesem Abschnitt wird das in der Einleitung bereits angeschnittene bilaterale Verhandlungsmodell mit vollständiger Information systematisch entwickelt. Da prinzipiell eine Reihe von Möglichkeiten der Modellierung von Verhandlungsprozessen besteht, muß zunächst begründet werden, warum gerade dieses Modell gewählt wurde. Da das Ziel darin besteht, später unvollständige Information einzuführen, erscheint es sinnvoll, Rahmenbedingungen zu wählen, die dies ohne allzu groBen technischen Aufwand zulassen. Auch wenn dadurch real existierende Verhandlungsprozeduren nur ansatzweise beschrieben werden können, sollte es dennoch möglich sein, die Gültigkeit der Argumentation von Coase vor dem Hintergrund der nicht-kooperativen Spieltheorie zu überprüfen. Da es hier in erster Linie darauf ankommt, die Wirkungen unvollständiger Information auf die Effizienz der Verhandlungslösung herauszuarbeiten, ist es wichtig, einen Verhandlungsprozeß zu untersuchen, der bei vollständiger Information zu pareto-optimalen Allokationen führt, um die Effekte von Informationsasymmetrien isoliert von anderen Allokationsstörungen analysieren zu können. Unter Berücksichtigung dieser Argumente erscheint die in diesem Kapitel getroffene Modellwahl gerechtfertigt.

\subsubsection{Eigentumsrechte und Verhandlungsmacht}

Wie Coase ausgeführt hat, kann es zwischen Schädiger und Geschädigtem nur dann zu Verhandlungen kommen, wenn die "Eigentumsrechte" klar definiert sind. In diesem Zusammenhang wird üblicherweise zwischen zwei Möglichkeiten einer Ausgestaltung der Eigentumsordnung unterschieden: Hat der Schädiger das Eigentumsrecht, dann ist es ihm erlaubt, die Produktionsmenge nach Belieben auszudehnen. Er wird dies bis zu dem Punkt tun, an dem die Herstellung zusätzlicher Mengeneinheiten keinen zusätzlichen Gewinn mehr bringt. Er wird also seinen indivi- 
duell optimalen Output gemäß der Bedingung $b^{\prime}(x)=0$ wählen. Wenn der Geschädigte will, daß der Schädiger die Produktion einschränkt, muß er sich diese Einschränkung erkaufen, indem er beispielsweise dem Unternehmen den entgangenen Gewinn ersetzt. Diese Form der Ausgestaltung von Eigentumsrechten wird gemeinhin als Laisser-Faire-Prinzip bezeichnet. Umgekehrt besteht die Möglichkeit, dem Geschädigten ein Vetorecht gegen die Aufnahme der Produktion einzuräumen. Bei dieser als Verursacherprinzip bezeichneten Eigentumsregelung muß sich der Schädiger das Recht zur Ausübung seiner Aktivität erst vom Geschädigten erkaufen. Zahlt der Verursacher nicht, so bleibt ihm die Herstellung des Gutes gänzlich untersagt.

Neben der Frage der Eigentumsrechte spielt bei Verhandlungen noch die "Verhandlungsmacht" eine wichtige Rolle. Während die Eigentumsordnung lediglich die Richtung des Zahlungsstroms zwischen Schädiger und Geschädigtem festlegt, ist die Verhandlungsmacht ausschlaggebend für die Höhe der Zahlungen. Ist beispielsweise der subjektive Wert, den ein bestimmtes Gut für den potentiellen Verkäufer hat, niedriger als der Wert, den der potentielle Käufer diesem Gut beimißt, so ist klar, daß beide ein Interesse am Zustandekommen eines Tausches haben. Unklar ist dagegen, zu welchem Preis das Gut den Besitzer wechseln soll. Es steht lediglich fest, daß dieser Preis nicht kleiner als die Wertschätzung des Verkäufers und nicht größer als die Wertschätzung des Käufers sein kann. Welcher Preis sich letztendlich durchsetzt, hängt von der Fähigkeit der Tauschpartner ab, sich jeweils einen möglichst hohen Anteil des durch die beiden Wertschätzungen eingegrenzten Tauschgewinns zu sichern. Diese Fähigkeit ist gemeint, wenn in Zukunft von Verhandlungsmacht die Rede ist.

Verhandlungsmacht spielte bereits implizit in den Beispielen, die in der Einführung zur Beschreibung der Grundidee privater Internalisierungsverhandlungen herangezogen wurden, eine Rolle. Dort wurde ohne weitere Begründung unterstellt, einer der beiden Verhandlungspartner könne sich den gesamten Internalisierungsgewinn aneignen. An dieser extremen Form von Verhandlungsmacht soll im folgenden aus zwei Gründen festgehalten werden: Erstens erspart man sich zusätzliche Komplikationen, weil man sich bei der Modellierung des Verhandlungsprozesses nicht mehr mit Fragen der Aufteilung des Wohlfahrtsgewinns auseinandersetzen muß. Dies scheint insofern gerechtfertigt, als hier Verteilungsfragen vorerst nicht thematisiert werden sollen. Zweitens sind solche "Ausbeutungslösungen" bei vollständiger Information bekanntlich immer effizient. Diese Eigenschaft sollte aber das 
Verhandlungsergebnis, wie oben begründet, aufweisen. Die Frage ist allerdings, wie eine solch starke Verhandlungsposition begründet werden kann.

In der Literatur wird in diesem Zusammenhang von der Möglichkeit der Unterbreitung sog. "Take-it-or-leave-it-Offerten" ausgegangen. Diese spezielle Form eines Verhandlungsspieles hat man sich folgendermaßen vorzustellen:

Einer der beiden Verhandlungspartner ist als erster am Zuge und kann dem anderen ein Vertragsangebot unterbreiten. Dieser hat im zweiten Zug nur die Option, das Angebot zu akzeptieren oder abzulehnen. Insbesondere hat er nicht die Möglichkeit, einen Gegenvorschlag zu machen. Falls das Angebot der offerierenden Partei akzeptiert wird, erfüllen beide Parteien die vereinbarten Vertragsbedingungen. Im Falle einer Ablehnung wird der Status quo, also der Zustand vor Verhandlungsbeginn, aufrecht erhalten.

Es liegt auf der Hand, daß die offerierende Partei unter diesen Umständen ein Angebot formulieren wird, das ihren eigenen Nutzen maximiert und der akzeptierenden Partei vom Internalisierungsgewinn nicht mehr zugesteht, als unbedingt erforderlich. Wenn man annimmt, daß ein Angebot bereits akzeptiert wird, falls es keine Verschlechterung gegenüber dem Status quo darstellt, dann reicht es aus, die akzeptierende Partei auf ihrem Reservationsnutzenniveau zu halten, das durch den Status quo determiniert ist. ${ }^{4}$ Bei dieser Form von Verhandlungsprozeß verfügt die offerierende Partei über die gesamte Verhandlungsmacht, weil sie sich den Internalisierungsgewinn komplett aneignen kann.

Die Verhandlungsstärke der offerierenden Partei ist hier offensichtlich auf die Unfähigkeit der akzeptierenden Partei, eine Gegenofferte zu unterbreiten, zurückzuführen. Diese Unfähigkeit wird meist als gegeben betrachtet, ohne sie näher zu begründen. Ein plausibler Begründungsansatz hierfür, der eigentlich eher typisch für die neuere Spieltheorie ist, findet sich schon bei Schelling (1956), wo Verhandlungsmacht als "the power to bind oneself" bezeichnet wird. ${ }^{5}$ Damit ist folgendes gemeint:

Die Möglichkeit, eine Take-it-or-leave-it-Offerte zu machen, besteht nur insoweit, als es der offerierenden Partei gelingt, glaubhaft zu machen, daß die Verhand-

\footnotetext{
${ }^{4}$ Es ist in der Literatur üblich, bei Indifferenz zwischen Angebot und Status quo davon auszugehen, daß das Angebot akzeptiert wird. Alternativ dazu könnte man der akzeptierenden Partei einen kleinen Anteil $\varepsilon$ am Internalisierungsgewinn zugestehen, um ihr die Zustimmung zu "versüßen". Dieses $\varepsilon$ würde man dann aber gegen Null konvergieren lassen, so daß das Resultat praktisch dasselbe ist.

${ }^{5}$ Siehe Schelling (1956, S. 282).
} 
lungen im Falle einer Ablehnung des Angebots ein für alle mal gescheitert sind. Diese Glaubwürdigkeit kann sie nur dadurch vermitteln, daß sie sich selbst von vornherein alle Chancen verbaut, auf eine eventuelle Gegenofferte einzugehen. Der bewußte Verzicht auf prinzipiell verfügbare Optionen, die für den Offerierenden vielleicht kaum weniger vorteilhaft gewesen wären als die Ausbeutungslösung, kann ihm helfen, die gesamte Verhandlungsmacht an sich zu reißen und sich den Tauschgewinn vollständig anzueignen. Das folgende Beispiel möge das Grundprinzip dieser Art von Selbstbindung erläutern:

Herr H. ist im Besitz einer Opernkarte für eine bereits ausverkaufte Vorstellung. Die Aufführung soll am 10.9. stattfinden. Da er mittlerweile kein Interesse mehr an dem Opernbesuch hat, will er die Karte an den Opernfan Herrn F. verkaufen. Er weiß, daß Herr F. bereit ist, dafür maximal DM 150 zu bezahlen. Die Frage ist, wie Herr H. von Herrn F. die DM 150 auch tatsächlich erhalten kann. Würde er ihm die Karte zu diesem Preis anbieten, würde Herr F. vielleicht ablehnen und ihm im Gegenzug nur DM 50 bieten. Darüber könnte Herr H. noch froh sein, denn dies entspräche, wie Herr F. weiß, zumindest dem Preis, den er selbst dafür zu zahlen hatte. Um einer solchen Gegenofferte auszuweichen bittet er deshalb einen Freund, Herrn F. die Karte für DM 150 anzubieten und sich keinesfalls mit weniger zufrieden zu geben. Lieber solle er das Geschäft platzen lassen. Danach begibt er sich ohne Angabe seines Ziels bis zum 11.9. auf eine Reise, die er ohnehin schon lange geplant hatte. Herr F. ist zwar über das Verhalten des Herrn H. ziemlich verärgert, entschließt sich aber doch, die Karte zu diesem Wucherpreis zu kaufen.

Dieses Beispiel zeigt, daß es prinzipiell möglich ist, sich durch geschickte Selbstbindung den Tauschgewinn komplett zu sichern, wenn man andere Möglichkeiten einer gleichmäßigeren Aufteilung absichtlich zerstört. Wichtig ist dabei, daß die Optionen des Verhandlungspartners nur beschränkt werden können, indem man sich eigene Möglichkeiten absichtlich verbaut und dies dem Verhandlungspartner auch klarmacht. Weitere Beispiele, wie Verhandlungsmacht durch Selbstbindung etabliert werden kann, finden sich bei Schelling. ${ }^{6}$ Wenn also im folgenden davon ausgegangen wird, einer der beiden Verhandlungspartner besitze die gesamte Verhandlungsmacht, dann wird implizit unterstellt, daß dieser Verhandlungspartner eine Take-it-or-leave-it-Offerte unterbreiten kann, weil er über entsprechende Möglichkeiten der Selbstbindung verfügt. Probleme, die sich ergeben, wenn beide Parteien solche Möglichkeiten haben, sollen hier nicht weiter diskutiert werden.

${ }^{6} \mathrm{Vgl}$. Schelling (1956, S. 287 ff.). 
Gemeinsam mit den beiden Varianten der Ausgestaltung der Eigentumsrechte müßten nun bei der Beschreibung der Ausgangslage zu Verhandlungsbeginn im Prinzip vier Konstellationen unterschieden werden: Der Schädiger hat das Eigentumsrecht und die gesamte Verhandlungsmacht, der Schädiger hat nur das Eigentumsrecht oder nur die gesamte Verhandlungsmacht und der Schädiger hat weder das Eigentumsrecht noch die Verhandlungsmacht. Es wird also hier davon ausgegangen, daß Verhandlungsmacht nicht automatisch an das Eigentumsrecht gekoppelt ist. Derartige Verbindungen, etwa daß der Eigentümer auch über die Verhandlungsmacht verfüge, mögen vielleicht empirisch beobachtbar sein, aus theoretischer Sicht sind sie aber nicht zwingend.

\subsubsection{Die Verhandlungslösung bei vollständiger Information}

Nach dieser einführenden Diskussion von Fragen des Eigentumsrechts und der Verhandlungsmacht kann nunmehr die Verhandlungslösung bei vollständiger Information ermittelt werden. Im folgenden wird überwiegend im Rahmen des Verursacherprinzips argumentiert: Der Geschädigte hat das Recht auf Unterlassung einer Beeinträchtigung und ist demnach als Verkäufer dieses Rechts auch Zahlungsempfänger. Der Schädiger befindet sich in der Rolle des Käufers und soll annahmegemäß über die gesamte Verhandlungsmacht verfügen.

Angesichts der geschilderten Ausgangslage kann die Verhandlungsprozedur nun wie folgt beschrieben werden: Der Verursacher schlägt dem Geschädigten vor Aufnahme der Produktion einen Kontrakt vor, in dem die angestrebte Produktionsmenge $\mathrm{x}$ und eine Transferzahlung $\mathrm{z}$ festgeschrieben sind. Der Geschädigte hat lediglich die Option, das Vertragsangebot zu akzeptieren oder abzulehnen. Durch diese Annahme erhält der Verhandlungsprozeß die Struktur eines Prinzipal-Agenten-Problems, bei dem der Schädiger die Rolle des Prinzipals, der Geschädigte die des Agenten einnimmt. Da wir, wie vorher erläutert, davon ausgehen, der Schädiger könne Take-it-or-leave-it-Offerten unterbreiten, kann er sich bei der Ermittlung des für ihn gewinnmaximalen Vertragsangebots auf diejenigen Kombinationen von Output und Transferzahlung konzentrieren, die dem Geschädigten gerade seinen Reservationsnutzen garantieren. Das Reservationsnutzenniveau ist bestimmt durch die geltende Eigentumsordnung, in unserem Fall also durch das Verusacherprinzip. Bei Zugrundelegung dieser Rechtslage kann der Geschädigte im Extremfall die 
Herstellung des Gutes durch sein Veto verhindern. Er erhält dann natürlich auch keine Transferzahlungen. Wenn man davon ausgeht, daß der Nutzen des Geschädigten als Differenz zwischen Transferzahlung und erlittenem Schaden ausgedrückt werden kann, ist sein Reservationsnutzen, unabhängig von seinem Schadenstyp, gleich Null.7 $\mathrm{Da}$ wir in diesem Abschnitt von vollständiger Information ausgehen, kann der Schädiger den Schadenskoeffizienten des Geschädigten annahmegemäß beobachten. Die Bedingung für die Akzeptanz eines "Kontrakts " $\{\mathrm{x} ; \mathrm{z}\}$, die der Schädiger zu beachten hat, lautet dann

$$
\mathrm{u}(\mathrm{x}, \mathrm{z}, \theta)=\mathrm{z}-\theta \mathrm{x}=0
$$

Unter dieser Nebenbedingung maximiert der Schädiger durch Wahl von (d.W.v.) $\mathrm{x}$ und $\mathrm{z}$ seinen Gewinn, der durch

$$
\pi(x, z)=b(x)-z
$$

gegeben ist. Nach Einsetzen der Nebenbedingung in die Zielfunktion reduziert sich das Optimierungsproblem des Schädigers auf

$$
\max \pi(x, \theta)=b(x)-\theta x
$$

d.W.v. $\quad x$.

Der Gewinn des Verursachers entspricht damit gerade dem gesamtwirtschaftlichen Wohlfahrtsgewinn, der dann maximiert wird, wenn die effiziente Produktionsmenge $\mathrm{x}^{*}(\theta)$ mit $\mathrm{b}^{\prime}\left(\mathrm{x}^{*}(\theta)\right)=\theta$ realisiert wird. Der Schädiger wird also dem Geschädigten das Vertragsangebot $\left\{\mathrm{x}=\mathrm{x}^{*}(\theta) ; \mathrm{z}=\theta \mathrm{x}^{*}(\theta)\right\}$ unterbreiten, durch das er sich den gesamten Wohlfahrtsgewinn aneignen kann. Gleichzeitig wird der externe Effekt

\footnotetext{
${ }^{7}$ Würde man vom Laisser-Faire-Prinzip ausgehen, dürfte der Schädiger das Produktionsniveau frei wählen. Er würde dem Geschädigten dann anbieten, gegen eine entsprechende Zahlung die Produktion einzuschränken. Bei der Formulierung seines Vertragsangebots müßte er dabei berücksichtigen, daß das Reservationsnutzenniveau des Geschädigten dem erlittenen Schaden bei Produktion des Laisser-Faire-Outputs entspricht und deshalb mit steigendem Schadenstyp sinkt. Das Problem der Abhängigkeit des Reservationsnutzens vom Schadenstyp spielt bei vollständiger Information noch keine Rolle. Im Fall unvollständiger Information ergeben sich jedoch formale Komplikationen.
} 
vollständig internalisiert. Der Geschädigte wird das Angebot akzeptieren, da er sich bei vollem Schadensersatz im Vergleich zum Status quo nicht verschlechtert.

Dieses einfache Beispiel zeigt, daß private Verhandlungen über die Internalisierung externer Effekte unter vollständiger Information auch in einem nicht-kooperativen Modellrahmen zu effizienten Ergebnissen führen können. ${ }^{8}$ So gesehen kann man Coases Behauptungen zumindest unter diesen speziellen Bedingungen als echtes "Theorem" bezeichnen. Es gibt natürlich noch weitere Möglichkeiten der Modellierung von Verhandlungen, die zu gesamtwirtschaftlich optimalen Ergebnissen führen. Beispielsweise könnte man das oben beschriebene Szenario dahingehend abwandeln, daß man annimmt, der Geschädigte habe das Eigentumsrecht und die gesamte Verhandlungsmacht. Er wäre dann in der Position des Prinzipals und könnte dem Schädiger seine Vertragsbedingungen diktieren. Er würde die erlaubte Produktionsmenge und die Ausgleichszahlung so bestimmen, da $\beta$ sein Nutzen maximal wird unter der Nebenbedingung, daß der Schädiger gerade noch auf die genannten Bedingungen eingeht. Dies ist dann der Fall, wenn sein Gewinn, wie im Falle einer Nichteinigung, Null ist. Das Nutzenmaximierungskalkül des Geschädigten lautet dann

$\max u(x, z, \theta)=z-\theta x$

d.W.v. x, z

u.d.N. $\pi(x, z)=b(x)-z=0$

bzw.

(2.3) $\max u(x, \theta)=b(x)-\theta x$

d.W.v. $x$.

Das Kalkül des Geschädigten ist demnach bei dieser Konstellation von Eigentumsrecht und Verhandlungsmacht identisch mit dem des Schädigers im zuvor betrachteten Fall und führt ebenfalls zur vollständigen Internalisierung des externen Effekts.

${ }^{8}$ Vgl. dazu auch Schweizer (1988), insbesondere Abschnitt 5. 
Der Unterschied liegt nur in der Verteilung des Wohlfahrtsgewinns, den sich in diesem Fall der Geschädigte vollständig aneignen kann.

Die Annahme extremer Verhandlungsmacht führt zugegebenermaßen zu Resultaten, die gemessen an der Realität unter Umständen etwas absurd erscheinen mögen. Dies gilt insbesondere für das zuletzt genannte Beispiel, das praktisch auf eine Art "Enteignung" des Schädigers durch den Geschädigten hinausläuft. Man könnte jedoch auf die Take-it-or-leave-it-Offerten auch ganz verzichten und andere Verhandlungsprozesse untersuchen, in denen der Internalisierungsgewinn aufgeteilt wird. Da sich aber in Verhandlungsspielen mit symmetrischer Information in aller Regel effiziente Ergebnisse einstellen, ${ }^{9}$ ist das oben verwendete Modell im Hinblick auf die Zielsetzung dieses Kapitels nicht schlechter als andere, die vielleicht realistischer, aber auch deutlich komplizierter sind. Es sollen ja auf möglichst einfache und einleuchtende Weise die Effekte unvollständiger Information auf die Effizienz privater Verhandlungen herausgearbeitet werden. Deshalb erscheint es sinnvoll, bei vollständiger Information einen Verhandlungsproze $B$ zu unterstellen, bei dem kein Zweifel an einer effizienten Vereinbarung besteht, da auf diese Weise die Ungültigkeit des Coase-Theorems bei unvollständiger Information im selben Modellrahmen umso deutlicher zu Tage tritt. Ein effizientes Verhandlungsergebnis ist sicherlich dann gewährleistet, wenn eine Partei die andere auf ihr Reservationsnutzenniveau drücken und sich die gesamten Wohlfahrtsgewinne aneignen kann. Diese Möglichkeit ist im oben beschriebenen Modell nur deshalb gegeben, weil die Parteien den Reservationsnutzen des Verhandlungspartners kennen: Die Gewinnfunktion des Schädigers war ohnehin als allgemein bekannt vorausgesetzt worden. Die Schadensfunktion des Geschädigten wurde zwar prinzipiell als zufallsabhängig betrachtet; da jedoch angenommen wurde, der Schädiger könne den Grenzschaden $\theta$ beobachten, ist diese Unsicherheit nur insofern von Bedeutung, als ex ante nicht klar ist, wie hoch der gesamtwirtschaftliche Wohlfahrtsgewinn nach Abschluß der Verhandlungen sein wird. Sicher ist aber, daß das Verhandlungsergebnis ex post für jedes mögliche $\theta$ paretooptimal sein wird, so daß vor Verhandlungsbeginn wieder ein durchschnittlicher Wohlfahrtsgewinn in Höhe von

(2.1) $\quad \mathrm{W}^{*}=\int_{\theta^{-}}^{\theta^{+}}[\mathrm{b}(\mathrm{x} *(\theta))-\theta \mathrm{x} *(\theta)] \mathrm{f}(\theta) \mathrm{d}(\theta)$

zu erwarten ist.

${ }^{9}$ Vgl. z.B. Rubinstein (1982). 


\subsection{Das Verhandlungsmodell mit unvollständiger Information}

In diesem Abschnitt wird untersucht, welche Effizienzwirkungen das Auftreten unvollständiger Information zwischen Schädiger und Geschädigtem im oben eingeführten Verhandlungsmodell hat. Wie im vorhergehenden Abschnitt wird wieder vom Verursacherprinzip ausgegangen. Der Schädiger soll wieder über die Verhandlungsmacht verfügen, kann also wieder die Vertragsbedingungen diktieren. Im Unterschied zum vorher beschriebenen Szenario wird nun die Annahme, der Schädiger könne den Grenzschaden $\theta$ des Geschädigten beobachten, aufgegeben. Stattdessen wird unterstellt, da $ß$ vor Verhandlungsbeginn nur der Geschädigte selbst sein wahres $\theta$ kennt, während der Schädiger lediglich weiß, da $B$ in der Grundgesamtheit aller möglichen Typen von Geschädigten mit der stetigen Wahrscheinlichkeitsfunktion $F(\theta)$ über das Intervall $T=\left[\theta^{-}, \theta^{+}\right]$verteilt ist. Für den Verursacher bedeutet dies, da $\beta$ er nicht mehr weiß, mit welchen Kombinationen von Produktionsmenge $x$ und Transferzahlung $\mathrm{z}$ er den Geschädigten auf sein Reservationsnutzenniveau drücken kann. Damit ist fraglich, ob der Schädiger immer noch in der Lage ist, sich den gesamten Wohlfahrtsgewinn anzueignen.

Im folgenden wird gezeigt, daß der Schädiger, wenn er seinen Gewinn maximieren will, einen Teil des Wohlfahrtsgewinns an den Geschädigten abtreten muß, um ihn zur Offenbarung seines wahren Grenzschadens zu bewegen. Aufgrund der Notwendigkeit dieser Zusatzzahlungen, die über einen reinen Schadensersatz hinausgehen, kommt es zu einer Abweichung der beim Schädiger anfallenden privaten Grenzkosten einer zusätzlichen Produktionseinheit von den gesamtwirtschaftlich relevanten Grenzkosten, die gleich dem Grenzschaden des Geschädigten sind. Das vereinbarte Produktionsniveau wird deshalb niedriger sein als im Wohlfahrtsoptimum.

\subsubsection{Methodische Grundlagen}

\subsubsection{Die Grundstruktur des "Mechanism-Design"-Ansatzes}

Das Problem des Schädigers besteht darin, unter der Bedingung asymmetrischer Information einen gewinnmaximalen Kontrakt $\{x, z\}$ zu finden. Den Lösungs- 
ansatz für dieses Problem bietet ein spezieller Literaturzweig der Spieltheorie, der sich mit einer bestimmten Art von Spielen mit unvollständiger Information befaßt und in der englischsprachigen Literatur als "Mechanism Design" bezeichnet wird. ${ }^{10}$ Diese Spiele sind ganz allgemein dadurch charakterisiert, daß ein Spieler, der Prinzipal, eine Entscheidung zu treffen hat, für die er Informationen benötigt, über die er selbst nicht verfügt. Bei diesen Informationen handelt es sich um bestimmte charakteristische Merkmale der übrigen Spieler, der Agenten. Die konkreten Ausprägungen dieser Merkmale sind nur den Agenten selbst bekannt. Da die vom Prinzipal zu treffende Entscheidung die Nutzenpositionen der Agenten in irgendeiner Weise beeinflußt, werden diese nicht ohne weiteres bereit sein, ihre privaten Informationen wahrheitsgemäß zu bekunden. Vielmehr werden sie versuchen, sich durch ihre Angaben möglichst große Vorteile zu verschaffen und deshalb u.U. die falschen Charakteristika übermitteln. Aufgabe des Prinzipals ist es, Anreizstrukturen zu finden, die die Agenten zur korrekten Angabe der benötigten Informationen bewegen. Auf der Grundlage dieser Informationen kann er dann eine Entscheidung fällen, die im Hinblick auf seine jeweilige Zielsetzung optimal ist.

Die Problemstellungen, in denen Mechanism Design Anwendung findet, lassen sich in zwei Gruppen unterteilen: Man unterscheidet auf der einen Seite Prinzipal-Agenten-Probleme mit nur einem Agenten, wie z.B. die Preisgestaltung durch einen Monopolisten, der die Zahlungsbereitschaft des Konsumenten nicht kennt (Maskin und Riley(1984)), die Regulierung eines Monopols mit unbekannter Kostenstruktur (Baron und Myerson (1982)) oder den in diesem Abschnitt analysierten Fall bilateraler Verhandlungen über externe Effekte bei asymmetrischer Information. Auf der anderen Seite sind Probleme mit mehreren Agenten zu nennen, wie z.B. die Konzeption von Mechanismen zur optimalen Bereitstellung öffentlicher Güter (z.B. Clarke (1971) oder Groves und Ledyard (1977b)), die optimale Gestaltung von Auktionen (z.B. Myerson (1981)), oder den im nächsten Kapitel zu behandelnden Fall einer dezentralen Internalisierung externer Effekte bei mehreren Geschädigten.

Die grundlegende Struktur dieser Spiele besteht aus drei Stufen und kann, gleich angewandt auf die hier untersuchte Problemstellung, wie folgt beschrieben werden: Auf der ersten Stufe schlägt der Schädiger dem Geschädigten einen "Mechanismus" vor. Unter einem Mechanismus versteht man ganz allgemein ein bestimmtes Spiel zwischen Schädiger und Geschädigtem, oder besser gesagt ein

10Für einen ausführlichen Überblick vgl. Fudenberg und Tirole (1991), Kap. 7. 
"Spielgerüst"."1 Dieses setzt sich zusammen aus einer Menge S von "Strategien" oder "Signalen", die dem Geschädigten in dem Spiel zur Verfügung stehen, einer "Outputfunktion" $\overline{\mathrm{x}}($.) und einer "Transferfunktion" $\overline{\mathrm{z}}($.). Die Menge S wählbarer Strategien wird dem Geschädigten vom Schädiger mitgeteilt. Ebenso wird er darüber informiert, welchen Output $\bar{x}(s)$ und welche Transferzahlung $\bar{z}(s)$ der Schädiger wählen wird, wenn er selbst eine bestimmte Strategie $s \in \mathrm{S}$ ergreift.

Die Strategiemenge $S$ ist auf dieser Ebene noch ein recht abstraktes Gebilde. Sie könnte beispielsweise mit der Menge $T$ aller möglichen Schadenskoeffizienten übereinstimmen. In diesem Fall wäre der Geschädigte aufgefordert, dem Schädiger einfach einen Schadensparameter aus T zu nennen. S kann aber auch viel kompliziertere Strategien enthalten. Dies wäre z.B. dann der Fall, wenn sich der Schädiger aus irgendwelchen Gründen für eine mehrstufige Spielstruktur entscheiden würde, in der er wiederholt mit dem Geschädigten kommunizieren müßte. Die Strategiemenge und damit die Spielstruktur ist nicht von vornherein festgelegt, sondern im Prinzip selbst Bestandteil der Optimierungsentscheidung des Schädigers.

Darin, daß der Schädiger als erster am Zug ist und den Mechanismus vorschlagen kann, kommt, ähnlich wie im Modell mit vollständiger Information, seine Verhandlungsmacht zum Ausdruck. Wenn er über die gesamte Verhandlungsmacht verfügt, kann er die "Spielregeln" nach seinen Zielvorstellungen gestalten, während der Geschädigte lediglich darüber entscheiden kann, ob er am Spiel teilnehmen will oder nicht. Diese Entscheidung erfolgt auf der zweiten Spielstufe.

Die Bereitschaft des Geschädigten, am Mechanismus teilzunehmen, hängt davon $\mathrm{ab}$, wie hoch der Nutzen, den er dabei erreicht, im Vergleich zu seinem Reservationsnutzen ist. Allgemein hängt sein Nutzenniveau von seinem Schadenstyp, von der Outputentscheidung $x$ und von der Transferzahlung $z a b$. Die letzteren beiden sind wiederum Funktionen der gewählten Strategie s. Bevor der Geschädigte signalisieren kann, ob er den Mechanismus akzeptiert oder nicht, muß er aus der Menge der zulässigen Signale das für ihn optimale auswählen. Diese Wahlentscheidung kann durch folgendes Nutzenmaximierungskalkül beschrieben werden:

\footnotetext{
${ }^{11}$ Ein Mechanismus kann strenggenommen nicht als Spiel bezeichnet werden, da zum Zeitpunkt seiner Konzeption die Auszahlungen der Spieler noch nicht feststehen (Vgl. Green und Laffont (1979, S. 34)). Gibbard (1973) hat stattdessen den Ausdruck "game form" geprägt, der hier mit "Spielgerüst" übersetzt wurde.
} 
(2.4) $\max u(\overline{\mathrm{x}}(\mathrm{s}), \overline{\mathrm{z}}(\mathrm{s}), \theta)$

d.W.v. $s$

u.d.N. $s \in S$.

Die Lösung $\mathrm{s}^{*}(\theta)$ dieses Kalküls ist die beste Antwort des Geschädigten in dem vom Schädiger konzipierten Mechanismus. Der maximale Nutzen des Geschädigten ist dann gegeben durch $\mathrm{u}\left[\overline{\mathrm{x}}\left(\mathrm{s}^{*}(\theta)\right), \overline{\mathrm{z}}\left(\mathrm{s}^{*}(\theta)\right), \theta\right]$. Da das Verursacherprinzip gilt und sein Reservationsnutzen deshalb gleich Null ist, wird er die Teilnahme am Mechanismus akzeptieren, wenn die Ungleichung

$$
\mathrm{u}\left[\overline{\mathrm{x}}\left(\mathrm{s}^{*}(\theta)\right), \overline{\mathrm{z}}\left(\mathrm{s}^{*}(\theta)\right), \theta\right] \geq 0
$$

erfüllt ist.

Im dritten und letzten Schritt des Spiels teilt der Geschädigte dem Verursacher sein optimales Signal mit, sofern er die Teilnahme am Mechanismus akzeptiert. Dieser implementiert dann entsprechend seiner Ankündigung das Produktionsniveau $\overline{\mathbf{x}}\left(\mathrm{s}^{*}(\theta)\right)$ und die Transferzahlung $\overline{\mathrm{z}}\left(\mathrm{s}^{*}(\theta)\right)$, woraus wiederum die Auszahlungen für die beiden Spieler folgen. Die Auszahlung für den Geschädigten beträgt mit Sicherheit $\mathrm{u}\left[\overline{\mathrm{x}}\left(\mathrm{s}^{*}(\theta)\right), \overline{\mathrm{z}}\left(\mathrm{s}^{*}(\theta)\right), \theta\right]$, die des Schädigers ist dagegen unsicher. Er kann zwar die Überlegungen des Geschädigten nachvollziehen und sich für jedes $\theta \in T$ dessen optimale Strategie und die resultierende Allokation $\{\overline{\mathrm{x}}, \overline{\mathrm{z}}\}$ ausrechnen. Da er aber zu Beginn des Spiels die konkrete Ausprägung von $\theta$ nicht kennt, kann er nur mit einem erwarteten Gewinn kalkulieren, der durch

$$
E(\pi)=\int_{\theta^{-}}^{\theta^{+}} \pi\left[\bar{x}\left(s^{*}(\theta)\right), \bar{z}\left(s^{*}(\theta)\right)\right] f(\theta) d \theta
$$

gegeben ist.

\subsubsection{Das Enthüllungsprinzip und seine Implikationen}

Ziel des Verursachers ist nun die Maximierung seines erwarteten Gewinns unter der Nebenbedingung, daß der Geschädigte den Mechanismus akzeptiert. In der 
oben beschriebenen Formulierung des Problems besteht allerdings auf den ersten Blick eine solche Fülle von Freiheitsgraden, daß eine Lösung nahezu unmöglich erscheint. So muß der Schädiger ja neben Output- und Transferfunktion auch die Strategiemenge $\mathrm{S}$ und damit die Spielstruktur bestimmen. Die Frage, die sich dann stellt, lautet, welche Spielstruktur das Erreichen eines absoluten Gewinnmaximums sichert. Die Aufgabe, bei gegebener Spielstruktur Output- und Transferfunktionen zu finden, die den Gewinn maximieren, erscheint lösbar. Wie aber ist zu verfahren, wenn die Spielstruktur selbst frei wählbar ist?

Einen Ausweg aus diesem Dilemma weist das in der Literatur so häufig zitierte "Revelation Principle" oder "Enthüllungsprinzip". Dieses Theorem, das auf Gibbard (1973) zurückgeht, von Myerson (1979) weiterentwickelt und in entsprechenden Abwandlungen für die unterschiedlichsten Formen von Mechanism-Design-Problemen bewiesen wurde, ermöglicht eine drastische Einschränkung der Anzahl zu untersuchender Mechanismen. ${ }^{12}$ Bezogen auf den hier behandelten Fall mit nur einem Agenten besagt es folgendes:

Jedes Ergebnis (Allokation und Auszahlungen), das der Prinzipal mit einem beliebigen Mechanismus, der vom Agenten akzeptiert wird, erreichen kann, kann er auch mit einem direkten Enthüllungsmechanismus erreichen.

Unter einem direkten Enthüllungsmechanismus versteht man einen Mechanismus, bei dem der Agent in einem einstufigen Kommunikationsprozeß seine private Information wahrheitsgemäß offenbart. Das Enthüllungsprinzip hat für unser Problem die Implikation, daß der Schädiger immer einen zum ursprünglichen Mechanismus äquivalenten finden kann, in dem er den Geschädigten einfach nach $\theta$ fragt und dieser der Wahrheit entsprechend antworten wird. Die Idee, die dem Beweis des Enthüllungsprinzips zugrunde liegt, wird im folgenden, bezogen auf das vorliegende Beispiel, kurz skizziert:

Ausgangspunkt der Argumentation ist ein beliebiger Mechanismus mit $\mathrm{S}$ als Menge zulässiger Strategien und mit $\overline{\mathbf{x}}(\mathrm{s})$ und $\overline{\mathrm{z}}(\mathrm{s})$ als Entscheidungs- bzw. Transferfunktion. Der Geschädigte wählt in diesem Mechanismus seine optimale Strategie $\mathrm{s}^{*}(\theta)$ nach dem Optimierungskalkül (2.4). Der Schädiger wählt daraufhin die Produktionsmenge $\bar{x}\left(s^{*}(\theta)\right)$ und die Transferzahlung $\bar{z}\left(s^{*}(\theta)\right)$, so daß der Geschädigte die Nutzenposition $u\left[\overline{\mathrm{x}}\left(\mathrm{s}^{*}(\theta)\right), \overline{\mathrm{z}}\left(\mathrm{s}^{*}(\theta)\right), \theta\right]$ erreicht. Der Geschädigte akzep-

${ }^{12}$ Vgl. z.B. Dasgupta, Hammond und Maskin (1979). 
tiert, wenn dieser Ausdruck mindestens den Wert Null annimmt. Dieses Ergebnis kann nun auch durch den folgenden Mechanismus erreicht werden:

Der Schädiger wählt als Menge zulässiger Strategien das Intervall $\mathrm{T}$ aller möglichen Schadenskoeffizienten, d.h. er fordert den Geschädigten auf, ein (möglicherweise falsches) $\tilde{\theta} \in \mathrm{T}$ anzugeben. Da ein rationaler Prinzipal den ursprünglichen Mechanismus durchschaut, kann er sich ausrechnen, welche Strategie $s^{*}(\theta)$ ein Geschädigter vom Typ $\theta$ ergreifen wird. Er kann deshalb aus dem ursprünglichen Mechanismus einen neuen konstruieren, indem er ankündigt, er werde unter Verwendung des signalisierten Grenzschadens diejenige Strategie $\mathrm{s}^{*}(\tilde{\theta})$ ermitteln, die für einen Geschädigten vom Typ $\tilde{\theta}$ im ursprünglichen Mechanismus optimal gewesen wäre. Die Produktionsmenge und die Transferzahlung werden dann nach den Funktionsvorschriften $x(\tilde{\theta})=\bar{x}\left(s^{*}(\tilde{\theta})\right)$ bzw. $z(\tilde{\theta})=\bar{z}\left(s^{*}(\tilde{\theta})\right)$ gewählt. Der Geschädigte erreicht in diesem modifizierten Mechanismus das Nutzenniveau $\mathrm{u}[\mathrm{x}(\tilde{\theta}), \mathrm{z}(\tilde{\theta}), \theta]=\mathrm{u}\left[\overline{\mathrm{x}}\left(\mathrm{s}^{*}(\tilde{\theta})\right), \overline{\mathrm{z}}\left(\mathrm{s}^{*}(\tilde{\theta})\right), \theta\right]$. Da der wahre Grenzschaden $\theta$ beträgt, ist wegen des Kalküls (2.4) $\mathrm{s}^{*}(\theta)$ das optimale Signal im ursprünglichen Mechanismus. Deswegen gilt die Ungleichung

$$
\mathrm{u}\left[\overline{\mathrm{x}}\left(\mathrm{s}^{*}(\tilde{\theta})\right), \overline{\mathrm{z}}\left(\mathrm{s}^{*}(\tilde{\theta})\right), \theta\right] \leq \mathrm{u}\left[\overline{\mathrm{x}}\left(\mathrm{s}^{*}(\theta)\right), \overline{\mathrm{z}}\left(\mathrm{s}^{*}(\theta)\right), \theta\right]
$$

und damit per Definition von $\mathrm{x}($.$) und \mathrm{z}($.

$$
\mathrm{u}[\mathrm{x}(\tilde{\theta}), \mathrm{z}(\tilde{\theta}), \theta] \leq \mathrm{u}[\mathrm{x}(\boldsymbol{\theta}), \mathrm{z}(\theta), \theta] .
$$

Das bedeutet, daß sich in diesem neuen, direkten Mechanismus eine Falschangabe nicht lohnt. Der modifizierte Mechanismus wirkt also enthüllend, was nicht allzu überraschend ist. Die realisierte Allokation und die Auszahlungen sind in beiden Mechanismen identisch, so daß auch die Akzeptanzbedingung erfüllt ist.

Weniger offensichtlich als der Beweis des Enthüllungsprinzips ist die Antwort auf die Frage nach den Anwendungsmöglichkeiten dieses Theorems. Kreps (1990) schreibt in diesem Zusammenhang: "It (the revelation principle) can be thought of as a statement about how actually to implement contracts. But it may be better to use it with greater circumspection as a tool of analysis for finding the limits of what outcomes can be implemented, without reference to how best to implement a particular outcome."13

${ }^{13}$ Vgl. Kreps (1990, S.696). 
Das heißt einerseits, daß der Schädiger prinzipiell einen direkten Mechanismus, in dem er den Geschädigten einfach nach dessen Grenzschaden befragt, als konkrete Spielform wählen kann. Wenn nämlich jedes Ergebnis eines beliebigen, noch so komplexen Mechanismus auch durch einen direkten Enthüllungsmechanismus implementierbar ist, dann heißt dies umgekehrt, daß durch andere als direkte Enthüllungsmechanismen keine anderen Ergebnisse erreichbar sind.

Andererseits deutet die leichte Einschränkung, die aus dem Zitat spricht, auf Probleme hin, die die Anwendung eines direkten Mechanismus unter Umständen bereiten kann. Eines dieser Probleme, nämlich das Problem multipler Nash-Gleichgewichte, wird noch im dritten Kapitel anzusprechen sein, wenn das Verhandlungsmodell mit mehreren Geschädigten behandelt wird. Ein direkter Enthüllungsmechanismus ist also nicht unbedingt der beste Weg, ein bestimmtes Ergebnis zu implementieren.

In der Hauptsache sollte das Enthüllungsprinzip deshalb als Theorem verstanden werden, das der Abgrenzung aller Kombinationen von Produktionsmenge und Transferzahlung dient, die überhaupt realisierbar sind: Wenn der Schädiger sicher sein will, bei der Suche nach dem optimalen Kontrakt sämtliche realisierbaren Spielergebnisse zu erfassen, dann muß er die x-z-Kombinationen, die er dem Geschädigten in Abhängigkeit seines Schadenstyps vorschlägt, so gestalten, daß dieser keine Veranlassung mehr hätte, seinen wahren Typ zu verleugnen, wenn er unmittelbar danach befragt würde. Ein Kontrakt, der diese Eigenschaft besitzt, wird als "enthüllend" oder auch "anreizverträglich" bezeichnet.

Die Tatsache, daß eine Beschränkung auf anreizverträgliche Kontrakte ausreicht, ist keineswegs trivial. Der Schädiger hat ja eigentlich kein unmittelbares Interesse an einer Enthüllung der verborgenen Information, sondern will seinen erwarteten Gewinn maximieren. Es wäre also durchaus denkbar, daß er mit einem Mechanismus, in dem der Geschädigte seinen Schaden nicht wahrheitsgemäß offenbart, einen höheren Gewinn erzielen könnte. Das Enthüllungsprinzip schließt diese Möglichkeit aus, sofern der Geschädigte den Mechanismus durchschaut. Der gewinnmaximale Kontrakt muß auf jeden Fall in der Menge aller anreizkompatiblen Kontrakte liegen. Das heißt aber nicht, daß die gewählte Spielstruktur unbedingt ein direkter Enthüllungsmechanismus sein muß. Der Schädiger kann vielmehr aus der Menge anreizverträglicher Kontrakte diejenige(n) Output- und Transferfunktion(en) auswählen, durch die sein Gewinn maximiert wird und sich dann gesondert darüber Gedanken machen, mit welcher Art von Spiel er diese Funktionen am besten im- 
plementieren kann. So gesehen ist die konkrete Spielstruktur für die folgenden Analysen eher von untergeordneter Bedeutung. Da im hier behandelten Fall mit nur einem Agenten die Anwendung eines direkten Enthüllungsmechanismus unproblematisch ist, wollen wir unseren Überlegungen bis auf weiteres diese Spielstruktur zugrundelegen. Erst in Kapitel 3, in dem das Modell mit mehreren Geschädigten behandelt wird, werden wir sehen, daß direkte Enthüllungsmechanismen multiple Gleichgewichte aufweisen können, die den Prinzipal u.U. vor erhebliche Schwierigkeiten stellen.

\subsubsection{Das Optimierungsproblem des Schädigers}

Nach diesen Vorüberlegungen läßt sich das Optimierungskalkül des Schädigers folgendermaßen formulieren: Die Zielfunktion lautet allgemein

(2.9) $E(\pi)=\int_{\theta^{-}}^{\theta^{+}} \pi[x(\theta), z(\theta)] f(\theta) d \theta$.

Diese ist durch Wahl der Funktionen $\mathrm{x}(\theta)$ und $\mathrm{z}(\theta)$ zu maximieren, wobei wegen des Enthüllungsprinzips nur solche $\mathrm{x}($.$) und \mathrm{z}($.$) in Frage kommen, die die Bedin-$ gung der Anreizverträglichkeit (AV) erfüllen. Nehmen wir an, der wahre Schadensparameter des Geschädigten sei $\theta$. Wenn er diesen korrekt offenbart, wählt der Schädiger den Output $\mathrm{x}(\theta)$ und die Transferzahlung $\mathrm{z}(\theta)$. Gibt er dagegen fälschlicherweise den Grenzschaden $\theta^{\prime}$ an, dann produziert der Schädiger die Menge $\mathbf{x}\left(\theta^{\prime}\right)$ und zahlt ihm als Entschädigung $z\left(\theta^{\prime}\right)$. Anreizverträglichkeit bedeutet, daß sich eine solche Falschangabe für kein $\theta^{\prime} \in T$ lohnen darf. Da der Schädiger a priori nicht weiß, mit welchem Typ von Geschädigten er es zu tun hat, muß diese Anforderung für alle möglichen Typen erfüllt sein. Formal ausgedrückt muß also

(AV) $\mathrm{u}[\mathrm{x}(\theta), \mathrm{z}(\theta), \theta] \geq \mathrm{u}\left[\mathrm{x}\left(\theta^{\prime}\right), \mathrm{z}\left(\theta^{\prime}\right), \theta\right] \quad \forall \theta, \theta^{\prime} \in\left[\theta^{-}, \theta^{+}\right]$

gelten.

Wenn (AV) erfüllt ist, dann ist zwar gesichert, daß es für den Geschädigten am besten ist, seinen wahren Schaden anzugeben. Es könnte aber sein, daß er selbst in diesem Fall ein negatives Nutzenniveau erreicht. Da das Verursacherprinzip gilt, wird er aber nur am Mechanismus teilnehmen wollen, wenn ihm mindestens ein 
Nutzenniveau von Null garantiert wird. Der Schädiger muß deshalb darauf achten, daß der Geschädigte bei korrekter Offenbarung unabhängig von seinem (dem Schädiger unbekannten) Schadenstyp einen nichtnegativen Nutzen realisiert. In dieser Anforderung, die als Bedingung individueller Rationalität bezeichnet wird, kommt die Freiwilligkeit privater Verhandlungen zum Ausdruck. Formal lautet diese Bedingung

(IR) $\mathrm{u}[\mathrm{x}(\theta), \mathrm{z}(\theta), \theta] \geq 0 \quad \forall \theta \in\left[\theta^{-}, \theta^{+}\right]$.

In der hier verwendeten spezifischen Modellformulierung kann das zu lösende Optimierungsprogramm wie folgt zusammengefaßt werden:

$\max \int_{\theta^{-}}^{\theta^{+}}[b(x(\theta))-z(\theta)] f(\theta) d \theta$

d.W.v. x(.), z(.)

u.d.N.

(AV) $u(\theta)=z(\theta)-\theta x(\theta) \geq z\left(\theta^{\prime}\right)-\theta z\left(\theta^{\prime}\right) \quad \forall \theta, \theta^{\prime} \in\left[\theta^{-}, \theta^{+}\right]$

(IR) $\mathrm{u}(\theta) \geq 0 \quad \forall \theta \in\left[\theta^{-}, \theta^{+}\right]$.

In der obigen Form ist das Optimierungsproblem des Schädigers nicht lösbar. Deshalb soll im folgenden gezeigt werden, daß die Bedingungen (AV) und (IR) durch äquivalente Bedingungen ersetzt werden können, die eine explizite Lösung ermöglichen. Dieser Äquivalenzbeweis wird in der Literatur meist auf sehr technischem Niveau erbracht, während der ökonomische Gehalt der einzelnen Beweisschritte häufig etwas zu kurz kommt. ${ }^{14}$ Deshalb soll in diesem Teilabschnitt versucht werden, die Argumentation durch zusätzliche Erläuterungen und graphische Darstellungen zu ergänzen.

${ }^{14} \mathrm{Vgl}$. dazu beispielsweise Myerson (1981), Baron und Myerson (1982) oder Myerson und Satterthwaite (1983). Eine relativ gute Darstellung findet man in Fudenberg und Tirole (1991, S.257 ff.). Dort wird der Sinn in der Literatur üblicher Standardannahmen besonders deutlich. 


\subsection{Die Monotonie der Outputfunktion}

Im ersten Schritt wird gezeigt, $\mathrm{da} \beta$ die Outputfunktion $\mathrm{x}(\theta)$ notwendigerweise monoton abnehmen muß, wenn die vorgeschlagenen Kontrakte anreizverträglich sein sollen. Dazu betrachte man zwei unterschiedliche Typen von Geschädigten mit den wahren Grenzschäden $\theta$ bzw. $\theta^{\prime}$. Kontrakte, die (AV) für beide Typen erfüllen sollen, müssen den Ungleichungen

$$
u(\theta)=z(\theta)-\theta x(\theta) \geq z\left(\theta^{\prime}\right)-\theta x\left(\theta^{\prime}\right)
$$

bzw.

$$
\mathrm{u}\left(\theta^{\prime}\right)=\mathrm{z}\left(\theta^{\prime}\right)-\theta^{\prime} \mathrm{x}\left(\theta^{\prime}\right) \geq \mathrm{z}(\theta)-\theta^{\prime} \mathrm{x}(\theta) \quad \forall \theta, \theta^{\prime} \in\left[\theta^{-}, \theta^{+}\right]
$$

genügen. Nach Umformung der beiden Ungleichungen erhält man

$$
\theta\left(x(\theta)-x\left(\theta^{\prime}\right)\right) \leq z(\theta)-z\left(\theta^{\prime}\right)
$$

und

$$
\theta^{\prime}\left(x(\theta)-x\left(\theta^{\prime}\right)\right) \geq z(\theta)-z\left(\theta^{\prime}\right)
$$

Durch Kombination dieser beiden Beziehungen unter Berücksichtigung der Definitionen von $u(\theta)$ und $u\left(\theta^{\prime}\right)$ ergibt sich

(2.10) $x\left(\theta^{\prime}\right)\left(\theta^{\prime}-\theta\right) \leq u(\theta)-u\left(\theta^{\prime}\right) \leq x(\theta)\left(\theta^{\prime}-\theta\right)$

woraus folgt, daß für $\theta^{\prime} \geq \theta \quad x\left(\theta^{\prime}\right) \leq x(\theta)$ und für $\theta^{\prime}<\theta \quad x\left(\theta^{\prime}\right) \geq x(\theta)$ sein muß. Damit ist gezeigt, da $(\mathrm{AV})$ nur erfüllt sein kann, falls die Produktionsmenge, die der Schädiger wählt, nicht zunimmt, wenn der Geschädigte einen höheren Kostenparameter angibt.

Warum dies so sein muß, wird deutlich, wenn man den Verlauf der Indifferenzkurven unterschiedlicher Typen von Geschädigten in einem Diagramm betrachtet, in dem auf der Abszisse die Produktionsmenge $\mathrm{x}$ und auf der Ordinate die Transferzahlung $z$ abgetragen wird. Die Indifferenzkurven für den $\theta$-Typ sind paral- 
lele Geraden der Form $\mathrm{z}=\mathrm{u}+\theta \mathrm{x}$, wobei $\mathrm{u}$ ein gegebenes Nutzenniveau darstellt. Dieses Nutzenniveau ist umso höher, je höher die Transferzahlung bei konstanter Produktionsmenge ist. Indifferenzkurven, die im Diagramm weiter oben liegen, bezeichnen also höhere Nutzenniveaus. Wenn man annimmt, daß $\theta^{\prime}<\theta$ ist, dann sind die Indifferenzkurven für den $\theta^{\prime}$-Typ flacher. Dieser Sachverhalt ist in Abbildung 5 dargestellt.

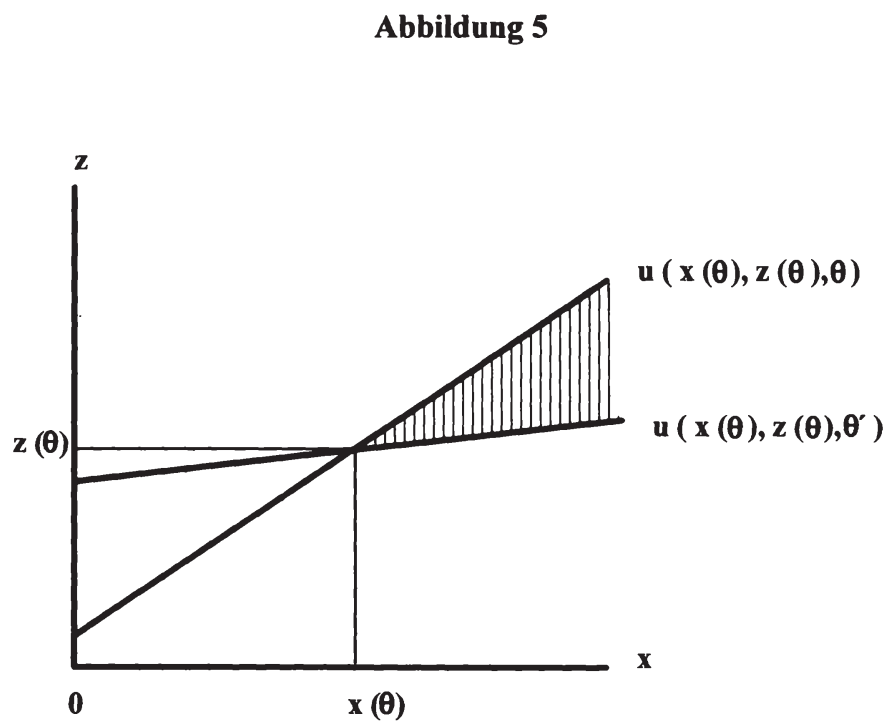

Wenn in dieser Abbildung der für den $\theta$-Typ bestimmte Kontrakt beispielsweise durch $\{x(\theta), z(\theta)\}$ gegeben ist, dann muß der Kontrakt $\left\{x\left(\theta^{\prime}\right), z\left(\theta^{\prime}\right)\right\}$ für den $\theta^{\prime}$-Typ auf oder oberhalb dessen Indifferenzkurve durch den Punkt $\{x(\theta), z(\theta)\}$ liegen, damit der $\theta^{\prime}$-Typ nicht ebenfalls auf den Kontrakt $\{x(\theta), z(\theta)\}$ zugreift. Gleichzeitig darf aber der Kontrakt für den $\theta^{\prime}$-Typ nicht oberhalb der Indifferenzkurve des $\theta$ Typs liegen, da dieser sonst $\left\{\mathrm{x}\left(\theta^{\prime}\right), \mathrm{z}\left(\theta^{\prime}\right)\right\}$ statt $\{\mathrm{x}(\theta), \mathrm{z}(\theta)\}$ wählen würde. Der Vertrag $\left\{x\left(\theta^{\prime}\right), z\left(\theta^{\prime}\right)\right\}$ kann deshalb in Abbildung 5 nur im schraffierten Bereich 
(einschließlich der Ränder) liegen. Das bedeutet, da $\beta$ in jedem Fall $x\left(\theta^{\prime}\right) \geq x(\theta)$ gelten muß. ${ }^{15} \mathrm{Da} \theta^{\prime}<\theta$ angenommen wurde, ist klar, daß $\mathrm{x}($.) monoton fallen muß.

\subsection{Die Bedingungen der individuellen Rationalität}

Im nächsten Schritt wird gezeigt, daß die Bedingung der individuellen Rationalität für alle Typen von Geschädigten mit $\theta<\theta^{+}$automatisch erfüllt ist, wenn der Kontrakt für den $\theta^{+}$- Typ individuell rational ist und die Verträge für alle Typen anreizverträglich sind. Wenn für den $\theta^{+}$-Typ (IR) gelten soll, dann darf sein Nutzenniveau nicht negativ sein, falls er $\theta^{+}$wahrheitsgemäß bekundet. Der Schädiger produziert in diesem Fall die Menge $x\left(\theta^{+}\right)$, so da $\beta$ der verursachte Schaden $\theta^{+} x\left(\theta^{+}\right)$ beträgt. Als Entschädigung wird $\mathrm{z}\left(\theta^{+}\right)$gezahlt. Falls also ein Geschädigter vom Typ $\theta^{+}$die Wahrheit sagt, muß sein Nutzenniveau $z\left(\theta^{+}\right)-\theta^{+} x\left(\theta^{+}\right) \geq 0$ sein. Da sich ein Geschädigter vom Typ $\theta<\theta^{+}$immer als $\theta^{+}$. Typ ausgeben und dadurch ein Nutzenniveau $z\left(\theta^{+}\right)-\theta x\left(\theta^{+}\right)>z\left(\theta^{+}\right)-\theta^{+} x\left(\theta^{+}\right)$erreichen kann, ist es dem Schädiger nicht möglich, einen solchen Typ auf das gleiche Nutzenniveau zu drücken wie einen $\theta^{+}$- Typ. Da aber alle Kontrakte anreizverträglich sein sollen, darf es sich nicht lohnen, einen falschen Kostenparameter anzugeben. Es muß also insbesondere $\mathrm{z}\left(\theta^{+}\right)-\theta \mathrm{x}\left(\theta^{+}\right) \leq \mathrm{z}(\theta)-\theta \mathrm{x}(\theta)$ für alle $\theta$ gelten, so daß ein $\theta$ - Typ zwangsläufig eine strikt positive Auszahlung erhalten muß. Die (IR) - Bedingungen sind damit für $\theta<\theta^{+}$nicht bindend und können im Optimierungskalkül des Schädigers vernachlässigt werden. ${ }^{16}$

An dieser Stelle wird deutlich, daß es dem Schädiger bei unvollständiger Information nicht mehr möglich ist, jeden Typ von Geschädigten auf seinem Reservationsnutzenniveau zu halten. Er wird also i.d.R. einen Teil der erzielbaren Wohlfahrtsgewinne an den Geschädigten abtreten müssen. Nur ein Geschädigter vom

${ }^{15}$ Derartige Monotonieeigenschaften sind typisch für die Charakterisierung anreizkompatibler Verträge. Ausschlaggebend dafür ist das Monotonieverhalten der Grenzrate der Substitution zwischen $\mathrm{x}$ und $\mathrm{z}$ in bezug auf $\theta$. Wenn diese streng monoton zu- oder, wie hier, abnimmt, können sich die Indifferenzkurven verschiedener Typen von Geschädigten nur einmal schneiden. Diese "single-crossing-property" ist der Grund für die globale Monotonie von x( $\theta)$. (Vgl. dazu Fudenberg und Tirole (1991, S. 258 f.)).

16Diese Argumentation ist nur unter der Annahme identischer Reservationsnutzenniveaus zulässig. Bei unterschiedlichen Reservationsnutzen können die (IR)-Bedingungen auch für andere Typen binden. 
Typ $\theta^{+}$wird im Gewinnmaximum des Schädigers über seinen Reservationsnutzen nicht hinauskommen. Der Schädiger könnte ja für den Fall, daß $z\left(\theta^{+}\right)-\theta^{+} x\left(\theta^{+}\right)>0$ wäre, die Entschädigung $z\left(\theta^{+}\right)$auf das Schadensersatzniveau $\theta^{+} x\left(\theta^{+}\right)$absenken, ohne dadurch die Anreizverträglichkeit der anderen Kontrakte zu beeinträchtigen. Es würde dadurch für die anderen Typen nur noch unattraktiver, sich als $\theta^{+}$- Typ auszugeben. Die einzige (IR)-Restriktion, die dann noch übrigbleibt, lautet

(2.11) $\mathrm{u}\left(\theta^{+}\right)=\mathrm{z}\left(\theta^{+}\right)-\theta^{+} \mathrm{x}\left(\theta^{+}\right)=0$.

\subsection{Anreizverträglichkeit und Informationsrenten}

Wie wir soeben gesehen haben, ist es dem Schädiger nicht mehr möglich, den Geschädigten auf seinem Reservationsnutzenniveau zu halten, es sei denn, sein Schadensparameter ist $\theta^{+}$. Damit ist klar, daß der Geschädigte, wenn er vom Typ $\theta$ $<\theta^{+}$ist, sich nicht mit einem bloßen Schadensersatz zufrieden geben muß, sondern einen bestimmten Anteil am Internalisierungsgewinn erhalten wird. Diesen Anteil bezeichnet man üblicherweise als "Informationsrente". Im folgenden geht es darum, festzustellen, wie hoch diese Informationsrente in Abhängigkeit des Schadenstyps sein muß, damit die Anreizverträglichkeit der Kontrakte gewährleistet ist.

In der Literatur findet man zwei unterschiedliche Methoden zur Ermittlung der Informationsrente. Zunächst soll die häufiger anzutreffende Methode beschrieben werden. ${ }^{17}$

Den Ausgangspunkt der Überlegungen bildet die zu (AV) äquivalente Ungleichung (2.10). Wenn man (2.10) durch $\left(\theta-\theta^{\prime}\right)$ dividiert, erhält man

$$
-x\left(\theta^{\prime}\right) \geq \frac{u(\theta)-u\left(\theta^{\prime}\right)}{\theta-\theta^{\prime}} \geq-x(\theta) \quad \text { für } \theta>\theta^{\prime}
$$

bzw.

$$
-x\left(\theta^{\prime}\right) \leq \frac{u(\theta)-u\left(\theta^{\prime}\right)}{\theta-\theta^{\prime}} \leq-x(\theta) \quad \text { für } \theta<\theta^{\prime} .
$$

${ }^{17}$ Vgl. z.B. Myerson (1981, S. 63f.) oder Baron und Myerson (1982, S. 916 f.). 
Bildet man bei diesen beiden Ungleichungen den Grenzwert für $\theta \rightarrow \theta^{\prime}$, so ergibt sich

$$
u^{\prime}\left(\theta^{\prime}\right)=d u\left(\theta^{\prime}\right) / d \theta^{\prime}=-x\left(\theta^{\prime}\right)
$$

als weitere notwendige Bedingung für eine anreizkompatible Vertragsgestaltung. ${ }^{18}$

Wenn man (2.12) auf beiden Seiten mit $\theta$ als Unter- und $\theta^{+}$als Obergrenze integriert, dann erhält man

$$
u(\theta)-u\left(\theta^{+}\right)=-\int_{\theta}^{\theta^{+}} u^{\prime}(t) d t=\int_{\theta}^{\theta^{+}} x(t) d t .19
$$

Setzt man in diese Beziehung die Definition $u(\theta)=z(\theta)-\theta x(\theta)$ ein und formt die resultierende Gleichung um, dann erhält man mit

$$
z(\theta)=\theta x(\theta)+\int_{\theta}^{\theta^{+}} x(t) d t+u\left(\theta^{+}\right)
$$

einen expliziten Zusammenhang zwischen Transfer- und Outputfunktion. ${ }^{20}$ Wenn man in (2.14) noch berücksichtigt, daß $\mathrm{u}\left(\theta^{+}\right)$, wie oben begründet, im Gewinnmaximum gleich Null sein muß, ergibt sich schließlich

(2.15) $\mathrm{z}(\theta)=\theta \mathrm{x}(\theta)+\int_{\theta}^{\theta^{+}} \mathrm{x}(\mathrm{t}) \mathrm{dt}$

${ }^{18}$ Genaugenommen gilt (2.12) nur, falls $x($.$) an der Stelle \theta^{\prime}$ stetig ist. In diesem Fall stimmen nämlich links- und rechtseitiger Grenzwert von $\mathrm{x}($.) an dieser Stelle überein, so daß auch die linksund rechtsseitigen Ableitungen von $u(),. \lim _{\theta \rightarrow \theta^{\prime}, \theta>\theta^{\prime}} \frac{u(\theta)-u\left(\theta^{\prime}\right)}{\theta-\theta^{\prime}}$ bzw. $\lim _{\theta \rightarrow \theta^{\prime}, \theta<\theta^{\prime}} \frac{u(\theta)-u\left(\theta^{\prime}\right)}{\theta-\theta^{\prime}}$, gleich sind. Dies ist die Voraussetzung für die Existenz von $\mathrm{u}^{\prime}\left(\theta^{\prime}\right)$. Ist $\mathbf{x}($.$) dagegen bei \theta^{\prime}$ nicht stetig, dann ist $u($.$) an dieser Stelle nicht differenzierbar.$

${ }^{19}$ Das Riemann-Integral existiert nur, wenn der Integrand höchstens abzählbar viele Sprungstellen aufweist (Vgl. Heuser (1991, S. 473)). Da x(.) monoton in $\theta$ verläuft, ist diese Voraussetzung hier erfüllt (Vgl. Heuser (1991, S. 240)). Falls bei x(.) Unstetigkeitsstellen auftreten sollten, kann in den Teilintervallen, in denen die Funktion stetig ist, integriert werden und die Summe der Teilintegrale gebildet werden.

20 Dieser Zusammenhang ermöglicht die Eliminierung der Transferfunktion aus dem Entscheidungskalkül des Schädigers und vereinfacht so die formale Behandlung des Problems beträchtlich. Dies ist auch der offensichtliche Grund dafür, warum die Separabilität der Nutzenfunktion des Geschädigten in der Literatur meist als Standardannahme vorausgesetzt wird. 
Diese Gleichung besagt, daß der Verursacher der Externalität einem Geschädigten vom Typ $\theta$ in einem anreizverträglichen Kontrakt zusätzlich zu seinem erlittenen Schaden $\theta x(\theta)$ eine Zahlung in Höhe von $\int_{\theta}^{\theta^{+}} x(t) d t$ anbieten muß. Die Funktion dieser Zahlung besteht darin, die private Information von Geschädigten mit niedrigeren Schadensparametern als $\theta^{+}$zu enthüllen.

Da bei dieser Art der Herleitung von (2.15) die ökonomische Interpretation des Terms $\int_{\theta}^{\theta^{+}} x(t) d t$ nicht besonders klar wird, soll noch eine weitere Variante angegeben werden, die in dieser Hinsicht um einiges transparenter ist. ${ }^{21}$

Betrachten wir dazu das Nutzenmaximierungskalkül des Geschädigten, wenn der Schädiger eine beliebige Outputfunktion $\mathrm{x}($.$) und eine beliebige Transferfunkti-$ on $z($.) vorgibt und fragen uns, welchen Schadenskoeffizienten $\hat{\theta}$ der Geschädigte angeben wird, wenn sein wahrer Schadenskoeffizient $\theta$ ist. Unter diesen Voraussetzungen ist seine Nutzenfunktion durch

$$
u(\theta, \hat{\theta})=z(\hat{\theta})-\theta x(\hat{\theta})
$$

gegeben. Diese ist durch Wahl von $\hat{\theta}$ zu maximieren, um die optimale Schadensmeldung zu bestimmen. Es ergibt sich

$$
z^{\prime}(\hat{\theta})-\theta x^{\prime}(\hat{\theta})=0
$$

als notwendige Bedingung für ein Nutzenmaximum. Aus dieser Gleichung kann im Prinzip das optimale Signal $\hat{\theta}(\theta)$ berechnet werden. Setzt man die Optimallösung in die Zielfunktion ein, dann erhält man mit

$$
\mathrm{v}(\theta)=\mathbf{u}(\hat{\theta}(\theta), \theta)=\mathrm{z}(\hat{\theta}(\theta))-\theta \mathbf{x}(\hat{\theta}(\theta))
$$

die indirekte Nutzenfunktion des Geschädigten, die seinen maximalen Nutzen bei optimaler Angabe von $\hat{\theta}$ beschreibt.

Man kann nun untersuchen, wie der maximale Nutzen mit dem wahren Schadenskoeffizienten $\theta$ variiert. Dazu differenziert man $v(\theta)$ und erhält

${ }^{21}$ Zur formalen Methodik vgl. auch Fudenberg und Tirole (1991, S. 264). Dort findet man aber leider ebenfalls keine befriedigende ökonomische Interpretation. 


$$
v^{\prime}(\theta)=\left[z^{\prime}(\hat{\theta}(\theta))-\theta x^{\prime}(\hat{\theta}(\theta))\right] d \hat{\theta} / d \theta-x(\hat{\theta}(\theta))
$$

Wenn man berücksichtigt, daß der Ausdruck in der eckigen Klammer wegen der notwendigen Bedingung für ein Nutzenmaximum gleich Null ist, ergibt sich schließlich

$(2.12)^{\prime} \mathrm{v}^{\prime}(\theta)=-\mathrm{x}(\hat{\theta}(\theta))$.

Diese Gleichung besagt, da $ß$ der Geschädigte bei optimaler Wahl seines Signals ein umso höheres Nutzenniveau realisieren kann, je niedriger sein wahrer Schadenskoeffizient ist. Damit ist klar, daß der $\theta^{+}$- Typ das niedrigste Nutzenniveau aller Typen erreichen wird. Diese Feststellung deckt sich mit den Überlegungen zur Relevanz der (IR)-Bedingungen. Im vorangegangenen Teilabschnitt wurde ja bereits gezeigt, daß nur der $\theta^{+}$- Typ auf sein Reservationsnutzenniveau gedrückt werden kann.

Mit Hilfe von (2.12)' kann nun die Differenz zwischen dem maximal erreichbaren Nutzenniveau eines $\theta^{+}$- Typs und dem eines $\theta$ - Typs ermittelt werden. Diese Nutzendifferenz erhält man, indem man den durch (2.12) beschriebenen marginalen Vorteil eines geringfügig niedrigeren Schadenskoeffizienten aufintegriert. Es errechnet sich ein Gesamtvorteil in Höhe von

$$
v(\theta)-v\left(\theta^{+}\right)=-\int_{\theta}^{\theta^{+}} v^{\prime}(t) d t=\int_{\theta}^{\theta^{+}} x(\hat{\theta}(t)) d t
$$

Dieser Vorteil ist nichts anderes anderes als die "Informationsrente", die zwangsläufig entsteht, weil der Schädiger den wahren Schadenskoeffizienten nicht beobachten kann. Da (2.13)' für beliebige Output- und Transferfunktionen gilt, gibt es keinen Kontrakt, mit dem der Schädiger das Auftreten solcher Informationsrenten verhindern könnte. Dies gilt insbesondere auch für einen enthüllenden Kontrakt, der gewährleisten soll, daß für den Geschädigten die Wahl des Signals $\hat{\theta}(\theta)=\theta$ optimal ist. Ein enthüllender Kontrakt muß also nach (2.13)' dem Geschädigten notwendigerweise eine Informationsrente in Höhe von $\int_{\theta}^{\theta^{+}} \mathrm{x}(\hat{\theta}(\mathrm{t})) \mathrm{dt}=\int_{\theta}^{\theta^{+}} \mathrm{x}(\mathrm{t}) \mathrm{dt}$ sichern, so $\mathrm{da} ß$ man schließlich wieder zu Gleichung (2.13) kommt, wenn man berücksichtigt, $\mathrm{da} ß$ bei korrekter Offenbarung von $\theta$ per Definition von $\mathrm{u}(\theta)$ die Beziehung $\mathrm{v}(\theta)=$ $\mathrm{u}(\theta)$ gilt. Die Gewährleistung der Informationsrente wird dadurch in die Tat umge- 
setzt, daß der Schädiger sich von vornherein vertraglich dazu verpflichtet, dem Geschädigten über einen Schadensersatz hinaus eine entsprechende Zusatzzahlung zu leisten. Darin liegt die ökonomische Bedeutung der Gleichungen (2.14) bzw. (2.15).

\section{Abbildung 6}

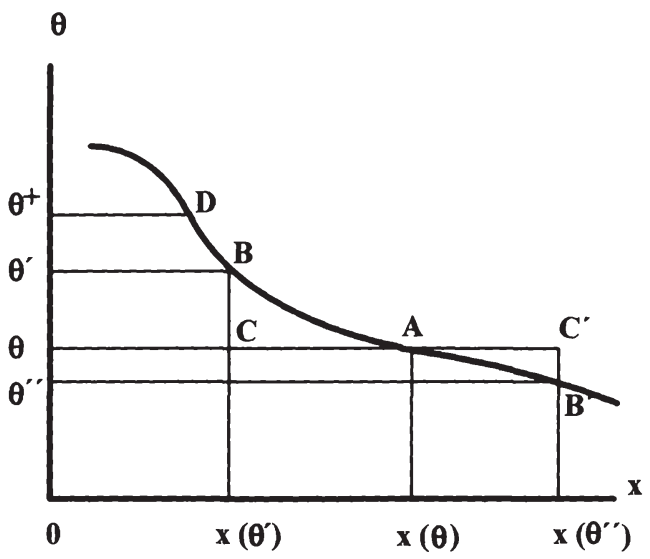

Die Wirkungsweise der Informationsenthüllungszahlung soll abschließend noch anhand von Abbildung 6 illustriert werden. Dabei wird auch deutlich, daß die Zahlung der oben berechneten Informationsrente zusammen mit der geforderten Monotonieeigenschaft der Outputfunktion nicht nur notwendige, sondern auch hinreichende Bedingung für Anreizverträglichkeit ist.

Betrachtet wird ein Geschädigter, dessen wahrer Grenzschaden durch $\theta$ gegeben sein soll. Gehen wir zunächst davon aus, daß der Schädiger die Enthüllungszahlung nicht leistet, sondern lediglich bereit ist, dem Geschädigten die angeblich erlittenen Schäden zu ersetzen. Es ist klar, daß der Geschädigte in diesem Fall keinen Anreiz hat, seinen wirklichen Schaden zu offenbaren. Er wird statt dessen übertreiben, um sich auf diese Weise einen Überschuß der erhaltenen Zahlungen über die tatsächlich erlittenen Schäden zu sichern. Gibt er z.B. fälschlicherweise den Grenzschaden $\theta^{\prime}$ an, dann produziert der Schädiger die Menge $\mathbf{x}\left(\theta^{\prime}\right)$ und leistet Schadensersatz in Höhe von $\theta^{\prime} x\left(\theta^{\prime}\right)$. Da sich die tatsächlichen Kosten des Geschädigten aber nur auf $\theta \mathbf{x}\left(\theta^{\prime}\right)$ belaufen, bringt ihm die Übertreibung einen Gewinn in Höhe von ( $\theta^{\prime}$ $\theta) \mathbf{x}\left(\theta^{\prime}\right)>0$. Ehrlichkeit hätte ihm dagegen nichts eingebracht. Der Anreiz, den 
Schaden zu übertreiben, wird durch die Enthüllungszahlung beseitigt: Wenn der Geschädigte seinen Schaden wahrheitsgetreu angibt, dann wird der Schädiger die Menge $x(\theta)$ produzieren und ihm einen Transfer in Höhe von $z(\theta)=\theta x(\theta)+$ $+\int_{\theta}^{\theta^{+}} x(t) d t$ zahlen. Sein Nettonutzen nach Abzug der tatsächlichen Schäden beträgt unter diesen Umständen $\int_{\theta}^{\theta^{+}} \mathrm{x}(\mathrm{t}) \mathrm{dt}$ statt 0 im vorhergehenden Fall. In Abbildung 6 entspricht dieser Nutzen betragsmäßig der Fläche $\theta^{+} \theta \mathrm{AD}$. Würde der Geschädigte dagegen wieder übertreiben und $\theta^{\prime}$ angeben, dann würde der Schädiger wegen der oben abgeleiteten Monotonieeigenschaft der Outputfunktion die niedrigere Produktionsmenge $x\left(\theta^{\prime}\right)$ wählen und dafür den Transfer $z\left(\theta^{\prime}\right)=\theta^{\prime} x\left(\theta^{\prime}\right)+\int_{\theta^{\prime}}^{\theta^{+}} x(t) d t$ zahlen. Der Nettonutzen, den der Geschädigte in diesem Fall erreichen könnte, beträgt $\left(\theta^{\prime}-\theta\right) x\left(\theta^{\prime}\right)+\int_{\theta^{\prime}}^{\theta^{+}} x(t) d t$. In Abbildung 6 wird dieser Betrag durch die Fläche $\theta^{+} \theta$ CBD beschrieben. Die Nutzenposition ist demnach bei korrekter Offenbarung um einen Betrag entsprechend der Fläche $\mathrm{ABC}$ höher als bei der Falschangabe.

Da die Anreizzahlung $\int_{\theta}^{\theta^{+}} \mathrm{x}(\mathrm{t}) \mathrm{dt}$ mit sinkendem $\theta$ zunimmt, wäre es prinzipiell denkbar, daß für den Geschädigten jetzt eine Untertreibung des Schadens attraktiv sein könnte. Man kann jedoch analog zur obigen Argumentation zeigen, $\mathrm{da} ß$ eine Untertreibung ebenfalls zu Nutzeneinbußen führt. Wenn der Geschädigte z.B. fälschlicherweise $\theta^{\prime \prime}$ statt $\theta$ angeben würde, würde er einen Nutzenverlust erleiden, der durch die Fläche $\mathrm{AB}^{\prime} \mathrm{C}^{\prime}$ gemessen werden kann.

Die Resultate dieses Teilabschnitts können nunmehr wie folgt zusammengefaßt werden: Wenn der Verursacher einer Externalität den Schaden, den er beim Geschädigten anrichtet, nicht beobachten kann, dann ist es ihm, anders als im Falle vollständiger Information, im allgemeinen nicht mehr möglich, den Geschädigten auf sein Reservationsnutzenniveau zu drücken und sich den gesamten Internalisierungsgewinn anzueignen. Würde er dies dennoch versuchen und dem Geschädigten nur einen Schadensersatz anbieten, würde dieser das bestehende Informationsgefälle zu seinen Gunsten ausnützen und sich durch eine Übertreibung des erlittenen Schadens Vorteile verschaffen. Will der Schädiger dies vermeiden, dann muß er über einen reinen Schadensersatz hinaus allen Typen von Geschädigten, die einen geringeren Schadenskoeffizienten aufweisen als $\theta^{+}$, eine Informationsrente zahlen, um die Offenbarung des wahren Ausmaßes der Schäden zu erreichen. Nur für den Fall, daß der Geschädigte den höchstmöglichen Schadenskoeffizienten aufweist und damit die Option einer Übertreibung von vornherein nicht hat, muß er sich mit dem 
reinen Schadensersatz zufriedengeben. Die asymmetrische Informationsverteilung zieht also für den Schädiger in fast allen Fällen zusätzliche Kosten nach sich, die er in seinem Gewinnmaximierungskalkül entsprechend berücksichtigen wird. Die entscheidende Frage, die sich an diese Feststellung anschließt, lautet dann, welche Konsequenzen diese Zusatzbelastung des Schädigers für die Effizienzeigenschaften der Verhandlungslösung hat.

\subsubsection{Die Verhandlungslösung bei unvollständiger Information}

Nachdem ausführlich dargelegt wurde, daß die Bedingung individueller Rationalität das Optimierungsproblem des Schädigers nur beschränkt, falls der Geschädigte vom Schadenstyp $\theta^{+}$ist und bewiesen wurde, daß die Anreizverträglichkeitsbedingungen genau dann erfültt sind, wenn die Outputfunktion monoton fällt und die Transferfunktion gemäß Gleichung (2.15) gestaltet wird, kann man nun dazu übergehen, die Optimallösungen für Output- und Transferfunktion zu ermitteln. Das Optimierungsproblem, das durch die obigen Überlegungen stark vereinfacht wurde, stellt sich nunmehr in folgender Form dar:

$$
\begin{aligned}
& \max E(\pi)=\int_{\theta^{-}}^{\theta^{+}}[b(x(\theta))-z(\theta)] f(\theta) d \theta \\
& \text { d.W.v. } x(.), z(.) \\
& \text { u.d.N. } z(\theta)=\theta x(\theta)+\int_{\theta}^{\theta^{+}} x(t) d t .
\end{aligned}
$$

Zusätzlich ist noch zu berücksichtigen, daß die Outputfunktion $\mathrm{x}(\theta)$ monoton fallen muß.

Die Nebenbedingung kann in die Zielfunktion eingesetzt werden, so daß der erwartete Gewinn des Schädigers nur noch von x(.) abhängt:

$$
E(\pi)=\int_{\theta^{-}}^{\theta^{+}}[b(x(\theta))-\theta x(\theta)] f(\theta) d \theta-\int_{\theta^{-}}^{\theta^{+}} \int_{\theta}^{\theta^{+}} x(t) f(\theta) d t d \theta
$$




$$
\begin{aligned}
& =\int_{\theta^{-}}^{\theta^{+}}[b(x(\theta))-\theta x(\theta)] f(\theta) d \theta-\int_{\theta^{-}}^{\theta^{+}} x(t) \int_{\theta^{-}}^{t} f(\theta) d \theta d t 22 \\
& =\int_{\theta^{-}}^{\theta^{+}}\{[b(x(\theta))-\theta x(\theta)] f(\theta)-x(\theta) F(\theta)\} d \theta .
\end{aligned}
$$

Dieser Ausdruck ist nun unter der Monotoniebeschränkung bezüglich $\mathrm{x}(.) \mathrm{zu}$ maximieren. In der Literatur wird die Zielfunktion üblicherweise zunächst unter Vernachlässigung der Monotoniebeschränkung optimiert. Anschließend werden dann Bedingungen angegeben, unter denen diese Vorgehensweise zulässig ist, weil die Monotonieeigenschaft ohnehin erfüllt ist. Wenn man so verfährt, erhält man als notwendige Bedingung für die optimale Wahl der Outputfunktion

$$
\left[b^{\prime}(x(\theta))-\theta\right] f(\theta)-F(\theta)=0
$$

Die Transferfunktion kann dann aus (2.15) berechnet werden.

Offensichtlich weicht bei unvollständiger Information die vom Schädiger gewählte Produktionsmenge von der durch $\mathrm{b}^{\prime}(\mathrm{x}(\theta))=\theta$ bestimmten effizienten Lösung in aller Regel ab. Dieser Umstand läßt sich leicht erklären, wenn man (2.16) ökonomisch interpretiert: Erhöht der Schädiger die Produktionsmenge, die er einem

${ }^{22}$ Beim Vertauschen der Integrationsreihenfolge ist zu beachten, daß sich die Integrationsgrenzen ändern, da die Untergrenze des inneren Integrals im zweiten Term der ersten Zeile selbst eine Funktion der Integrationsvariable des äußeren Integrals ist (Vgl. Shapiro und Whitney (1967, S. $406 \mathrm{ff}$.)). Es gilt allgemein $\int_{\theta^{-}}^{\theta^{+}} \int_{\theta}^{\theta^{+}} \mathrm{g}(\theta, \mathrm{t}) \mathrm{dt} d \theta=\int_{\theta^{-}}^{\theta^{+}} \int_{\theta^{-}}^{\mathrm{t}} \mathrm{g}(\theta, \mathrm{t}) \mathrm{d} \theta \mathrm{dt}$. Dies wird klar, wenn man sich den Bereich, über den integriert wird, in einem t- $\theta$-Diagramm veranschaulicht: Auf der linken Seite der obigen Gleichung wird zuerst über $t$ integriert, wobei die obere Integrationsgrenze durch die Konstante $\theta^{+}$, die Untergrenze durch die Funktion $t=\theta$ gebildet wird. Das äußere Integral über $\theta$ läuft von $\theta^{-}$bis $\theta^{+}$. Der gesamte Integrationsbereich ist also durch das schraffierte Dreieck in untenstehender Abbildung gegeben. Wenn man dagegen zuerst über $\theta$ und dann über $t$ integriert, muß das innere Integral von der Untergrenze $\theta^{-}$bis zur Obergrenze $\theta=t$ laufen, damit die Integrationsbereiche in beiden Fällen übereinstimmen.

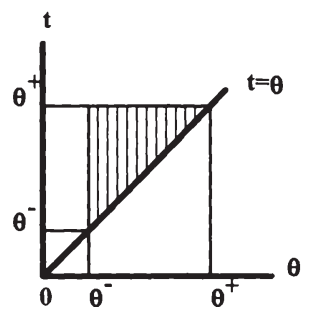


Geschädigten vom Typ $\theta$ vorschlägt, um eine marginale Einheit, so erzielt er einerseits einen Bruttozusatzgewinn in Höhe von $b^{\prime}(x(\theta))$. Dieser Term mißt auch den gesamtwirtschaftlichen Bruttozusatznutzen einer marginalen Erhöhung von $\mathbf{x}(\theta)$. Andererseits steigen auch die vom Schädiger zu leistenden Transferzahlungen, und zwar in zweifacher Hinsicht: Zum einen muß, was die Zahlung an einen Geschädigten vom Typ $\theta$ betrifft, dessen Schadensersatzkomponente $\theta x(\theta)$ um $\theta$ erhöht werden, so daß der Schädiger nach Abzug dieser Kosten nur noch einen zusätzlichen Gewinn in Höhe von $b^{\prime}(x(\theta))$ - $\theta$ erzielen kann. Dieser Betrag, der auch den gesamtwirtschaftlichen Nettonutzenzuwachs widerspiegelt, ist mit der Wahrscheinlichkeitsdichte $\mathrm{f}(\theta)$ zu gewichten. ${ }^{23}$ Darüber hinaus erhöht sich aber auch die Informationsrentenzahlung $\int_{\theta}^{\theta^{+}} x(t) d t$ für jeden Geschädigten, dessen Schadenstyp im Intervall $\left[\theta^{-}, \theta\right]$ liegt, um eine marginale Einheit. Diese Zusatzzahlung ist mit der Wahrscheinlichkeit für das Auftreten eines solchen Typs, also mit $F(\theta)$, zu gewichten. Gleichung (2.16) besagt dann, daß im individuellen Gewinnmaximum die erwartete Zunahme des Bruttogewinns mit den erwarteten zusätzlichen Transferzahlungen übereinstimmen muß.

Damit ist klar, warum der Schädiger im Vergleich zur effizienten Lösung fast immer ein zu niedriges Aktivitätsniveau wählt: Die Notwendigkeit, Informationsenthüllungszahlungen zu leisten, verursacht auf Seiten des Schädigers zusätzliche Kosten, die aus gesamtwirtschaftlicher Sicht irrelevant sind, weil sie lediglich eine Umverteilung der Wohlfahrtsgewinne zugunsten des Geschädigten darstellen. Die asymmetrische Informationsverteilung führt also zu einer Divergenz zwischen den einzel- und gesamtwirtschaftlichen Grenzkosten einer Ausdehnung von $\mathrm{x}$ und wird somit zur Ursache für das Versagen privater Verhandlungen über externe Effekte. Nur für den Fall, daß es sich bei dem Geschädigten um einen $\theta^{-}$-Typ handelt, setzt sich die effiziente Lösung durch. Der Grund dafür liegt auf der Hand: Bei einer marginalen Erhöhung von $\mathbf{x}\left(\theta^{-}\right)$muß nur die Informationsrente für den $\theta^{-}$-Typ erhöht werden. Die Wahrscheinlichkeit für dessen Auftreten ist aber $F\left(\theta^{-}\right)=0$, so daß nur in diesem Fall, wie sich auch formal aus (2.16) ergibt, das Produktionsniveau paretooptimal ist.

\footnotetext{
${ }^{23}$ Strenggenommen beschreibt diese Argumentation nicht die Effekte einer Erhöhung der Produktionsmenge exakt für den $\theta$ - Typ, da die Wahrscheinlichkeit für dessen Auftreten Null ist. Eine Veränderung von $\mathbf{x}(\theta)$ kann somit keine Auswirkungen auf den erwarteten Gewinn des Schädigers haben. Mathematisch korrekt wäre es, von einer marginalen Erhöhung von $x$ im Intervall $[\theta, \theta+\varepsilon]$ mit $\varepsilon \rightarrow 0$ zu sprechen (Vgl. Fudenberg und Tirole (1991, S. 265)).
} 
Nun bleibt noch zu klären, unter welchen Bedingungen die bisher vernachlässigte Monotonierestriktion wirklich ignoriert werden darf. Dazu formt man (2.16) um zu

$(2.16)^{\prime} b^{\prime}(x(\theta))=\theta+F(\theta) / f(\theta)$.

An dieser Formulierung der Optimalitätsbedingung wird deutlich, daß $\mathrm{x}(\theta)$ wegen $\mathrm{b}^{\prime \prime}(\mathrm{x})<0$ nur dann monoton fallt, wenn die rechte Seite monoton zunimmt. Hinreichend dafür ist, da $B$ der Quotient $F(\theta) / f(\theta)$ monoton zunimmt. Diese Eigenschaft ist glücklicherweise bei den gebräuchlichsten stetigen Wahrscheinlichkeitsverteilungen, wie z.B. Gleich-, Normal-, $\chi^{2}$ - und Exponentialverteilung erfüllt, so da $\beta$ es bei konkreten Anwendungen, in denen spezielle Verteilungsannahmen getroffen werden müssen, sinnvoll erscheint, sich auf diese Fälle zu beschränken. ${ }^{24}$

\subsection{Ein Beispiel}

In diesem Abschnitt soll abschließend noch die Funktionsweise des oben beschriebenen Mechanismus sowie die ökonomische Intuition, die hinter den abgeleiteten Ergebnissen steckt, anhand eines einfachen Beispiels graphisch verdeutlicht werden. Dabei wird davon ausgegangen, da $\beta \quad$ im Intervall $[0,1]$ gleichverteilt ist. Die Wahl der Intervallgrenzen erleichtert lediglich die graphische Darstellung, ist aber ansonsten nicht kritisch. Die spezielle Verteilungsannahme dient einerseits ebenfalls der Vereinfachung, kann andererseits aber auch in gewisser Weise inhaltlich motiviert werden: Wenn der Schädiger keinerlei Anhaltspunkte über die konkrete Gestalt der Verteilungsfunktion hat, erscheint es nicht unplausibel, alle $\theta \in$ $[0,1]$ für gleich

wahrscheinlich zu halten. In diesem Fall $m u \beta$ die Gleichverteilung von $\theta$ angenommen werden. Es soll also gelten

\footnotetext{
${ }^{24}$ Vgl. z.B. Fudenberg und Tirole (1991, S. 267). Es existieren auch Lösungsverfahren für Probleme, in denen die Monotoniebeschränkung bindet (Vgl. z.B. Myerson (1981)). In solchen Fällen erhalten typischerweise unterschiedliche Typen von Geschädigten gleiche Kompensationszahlungen. Dieses Problem soll aber hier nicht weiter verfolgt werden.
} 


$$
f(\theta)=\left\{\begin{array}{lc}
1 & \text { für } 0 \leq \theta \leq 1 \\
0 & \text { sonst }
\end{array}\right.
$$

bzw.

$$
F(\theta)=\left\{\begin{array}{lc}
0 & \text { für } \theta<0 \\
\theta & \text { für } 0 \leq \theta \leq 1 \\
1 & \text { sonst. }
\end{array}\right.
$$

Daraus folgt $F(\theta) / f(\theta)=\theta$. Weiterhin wird unterstellt, da $\beta$ die Grenzgewinnfunktion linear ist mit $b^{\prime}(x)=a-c x$. Die effiziente Produktionsmenge in Abhängigkeit vom jeweiligen Schadenstyp ist dann bestimmt durch a - $c x=\theta$, während der Output bei der Verhandlungslösung gemäß (2.16)' aus $\mathrm{a}-\mathrm{cx}=2 \theta$ bzw. $(\mathrm{a}-\mathrm{cx}) / 2=\theta$ folgt. Bezeichnet man die effiziente Lösung wieder mit $x^{*}$ und die Verhandlungslösung mit $\mathrm{x}_{\mathrm{v}}$, so ergibt sich

$$
x^{*}(\theta)=\left\{\begin{array}{cc}
(a-\theta) / c & \text { für } \theta<a \\
0 & \text { sonst }
\end{array}\right.
$$

bzw.

$$
x_{v}(\theta)=\left\{\begin{array}{cc}
(a-2 \theta) / c & \text { für } \theta<a / 2 \\
0 & \text { sonst. }
\end{array}\right.
$$

Um sowohl bei der effizienten Lösung als auch bei der Verhandlungslösung für alle $\theta \in[0,1]$ strikt positive Produktionsmengen zu erhalten, wird $a / 2>1$ angenommen. Auch diese Annahme dient lediglich der Vereinfachung späterer Berechnungen.

Abbildung 7 zeigt, wie die jeweiligen Outputs graphisch ermittelt werden können. Die Produktionsmengen $x^{*}(\theta)$ bzw. $x_{v}(\theta)$ resultieren aus den Schnittpunkten der Grenzgewinnfunktion a - cx bzw. der Geraden ( $\mathrm{a}$ - cx)/2 mit einer Parallelen zur Abszisse mit Ordinatenabschnitt $\theta$. Man sieht, da $\beta$ die Produktionsmenge $\mathrm{x}_{\mathrm{v}}(\theta)$ für alle $\theta$ mit Ausnahme von $\theta=0$ niedriger ist als die effiziente Menge $x^{*}(\theta)$. Der Wohlfahrtsverlust, der sich durch diese Abweichung ex post ergibt, falls der Ge- 
schädigte vom Typ $\theta$ ist, wird durch das schraffierte Dreieck beschrieben. Er berechnet sich zu

$$
\mathrm{v}(\theta)=(1 / 2)\left[\mathrm{x}^{*}(\theta)-\mathrm{x}_{\mathrm{v}}(\theta)\right]\left[\mathrm{b}^{\prime}\left(\mathrm{x}_{\mathrm{v}}(\theta)\right)-\theta\right]=(1 / 2)(\theta / \mathrm{c}) \theta=\theta^{2} / 2 \mathrm{c},
$$

wie leicht aus Abbildung 7 ersichtlich ist.

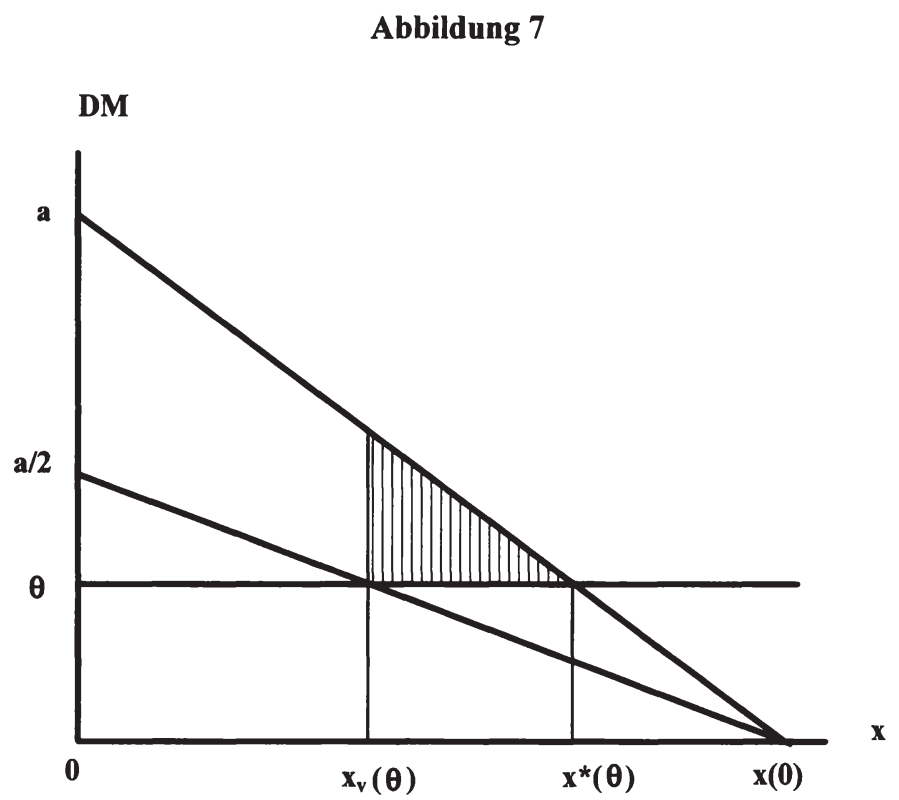

Der erwartete Wohlfahrtsverlust beträgt dann

$$
V=\int_{\theta^{-}}^{\theta^{+}} v(\theta) f(\theta) d \theta=\int_{0}^{1}\left(\theta^{2} / 2 c\right) d \theta=1 / 6 c
$$

Abbildung 8 zeigt, wie sich das Entscheidungsproblem aus der Sicht des Schädigers darstellt. Wenn der Geschädigte vom Typ $\theta$ ist, dann wählt der Schädiger gemäß Bedingung (2.16)' die Produktionsmenge $\mathrm{x}_{\mathrm{v}}(\theta)$. Der Bruttogewinn, den er dabei erzielt, wird durch die Fläche $0 \mathrm{x}_{\mathrm{v}}(\theta) \mathrm{Pa}$ unter der Grenzgewinnkurve beschrieben. Die Transferzahlung an den Geschädigten setzt sich zusammen aus der Schadenser- 
satzkomponente $\theta \mathrm{x}_{\mathrm{v}}(\theta)$, dargestellt durch die Fläche $0 \mathrm{x}_{\mathrm{v}}(\theta) \mathrm{Q} \theta$, und der Informationsrente $\int_{\theta}^{1} x_{v}(t) d t$, dargestellt durch die Fläche $\theta Q R 1$. Der verbleibende Nettogewinn wird damit durch die Fläche $1 \mathrm{RQPa}$ repräsentiert.

\section{Abbildung 8}

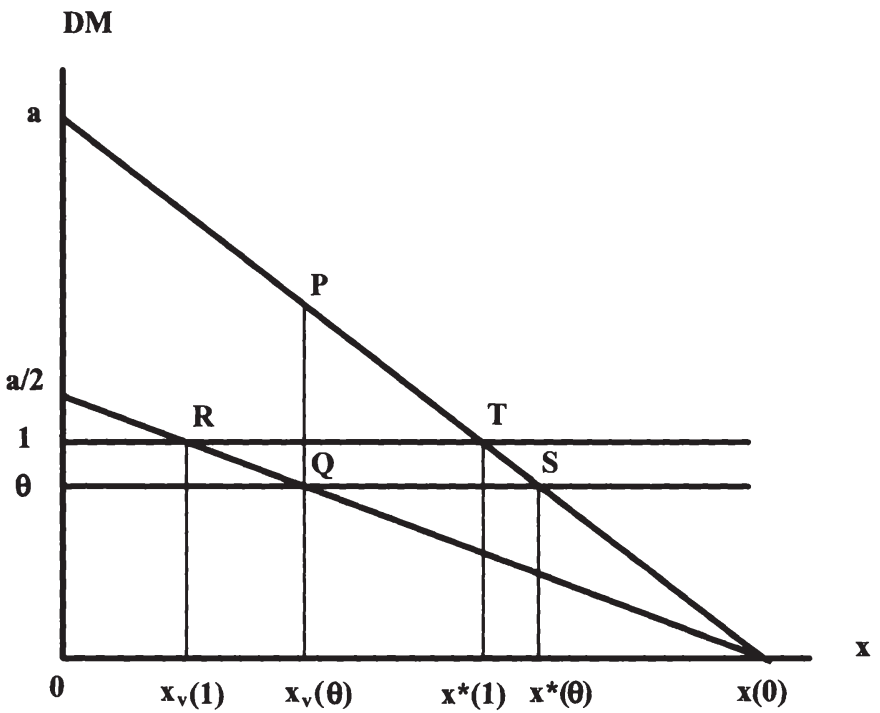

Es ist instruktiv, zum Vergleich den Gewinn zu betrachten, den der Schädiger erzielen würde, wenn er die effiziente Outputfunktion $x^{*}($.$) implementieren wollte.$ Er müßte dazu nur durch höhere Transferzahlungen die Anreizverträglichkeit der jeweiligen Kontrakte sichern. Die Schadensersatzkomponente für einen $\theta$-Typ müßte in diesem Fall $\theta \mathrm{x}^{*}(\theta)$ (Fläche $\left.0 \mathrm{x}^{*}(\theta) \mathrm{S} \theta\right)$ und die Informationsrente $\int_{\theta}^{1} x^{*}(t) d t$ (Fläche $\theta \mathrm{ST} 1$ ) betragen. Der Nettogewinn, der sich unter diesen Umständen ergäbe, entspricht in Abbildung 8 der Fläche 1Ta, wäre also offensichtlich höher als bei Implementation der Outputfunktion $\mathrm{x}_{\mathrm{v}}($.). Für "kleine" $\theta$ ist für den Schädiger dagegen die Outputfunktion $\mathrm{x}_{\mathrm{v}}($.) günstiger. Wäre der Geschädigte beispielsweise vom Typ $\theta=0$, würde der Schädiger sowohl bei Implementation von $x^{*}($.$) als auch bei$ 
Implementation von $\mathrm{x}_{\mathrm{v}}($.$) die Menge \mathrm{x}(0)$ produzieren. Im Falle der Implementation von $x^{*}($.$) ergäbe sich bei der Menge \mathrm{x}(0)$ der gleiche Nettogewinn wie bei der Menge $x^{*}(\theta)$. Dies gilt im übrigen für alle $\theta \in[0,1]$, wie man leicht anhand der Graphik nachprüfen kann. Der Nettogewinn bei Implementation von $\mathrm{x}_{\mathrm{v}}($.) entspricht jetzt der Fläche $1 \mathrm{Rx}(0) \mathrm{a}$ und ist damit um die Fläche $\mathrm{Rx}(0) \mathrm{T}$ höher als bei Implementation der Outputfunktion $x^{*}($.). Da der Schädiger $\theta$ aber nicht beobachten kann, kommt es nicht auf den Ex-Post-Gewinn nach Bekanntwerden des Schadenstyps an. Entscheidend ist, welche Outputfunktion ihm im Durchschnitt einen höheren Gewinn liefert.

Der Ex-Post-Gewinn bei Implementation von $x^{*}($.$) ist, wie gesagt, unabhängig$ von $\theta$ und ergibt sich auf einfache Weise durch Berechnung der Dreiecksfläche $1 \mathrm{Ta}$ in Abbildung 8:

$$
\pi^{*}=\pi\left(\mathrm{x}^{*}(\theta)\right)=(1 / 2)(\mathrm{a}-1) \mathrm{x}^{*}(1)=(1 / 2 \mathrm{c})(\mathrm{a}-1)^{2}
$$

Der Ex-Post-Gewinn bei Implementation von $\mathrm{x}_{\mathrm{v}}($.$) kann ebenfalls mit Hilfe$ von Abbildung 8 ermittelt werden. Man erhält nach einfachen Rechnungen

$$
\begin{aligned}
& \pi_{v}=\pi\left(x_{v}(\theta)\right)=\int_{0}^{x_{v}(1)}(a-c x-1) d x+\int_{x_{v}(1)}^{x_{v}(\theta)}(1 / 2)(a-c x) d x= \\
& =\pi^{*}+(1 / c)\left(1 / 2-\theta^{2}\right) .25
\end{aligned}
$$

Der Verlauf dieser beiden Gewinnfunktionen ist in Abbildung 9 dargestellt. Man sieht, da $B \pi_{v}$ lediglich für $\theta>\sqrt{1 / 2}$ kleiner ist als $\pi^{*}$. Die Differenz der beiden Gewinne beträgt im statistischen Durchschnitt

$$
D=\int_{\theta^{-}}^{\theta^{+}}\left(\pi_{v}(\theta)-\pi^{*}(\theta)\right) f(\theta) d \theta=\int_{0}^{1}(1 / c)\left(1 / 2-\theta^{2}\right) d \theta=1 / 6 c
$$

Diese Differenz entspricht in Abbildung 9 der Differenz der Flächen PQT und QRS. Damit ist gezeigt, daß die effiziente Outputfunktion $x^{*}($.$) im Prinzip implementier-$ bar wäre, daß aber die Implementation von $\mathrm{x}_{\mathrm{v}}($.) für den Schädiger günstiger ist. Dies liegt daran, daß bei der Implementation von $x^{*}($.) die zu zahlende Informati-

$25 \mathrm{Man}$ beachte, daß unter der oben getroffenen Annahme a/2>1 sowohl $\pi^{*}$ als auch $\pi_{\mathrm{v}}$ für alle $\theta \in$ $[0,1]$ positiv ist. 
onsrente für Typen mit "kleinen" $\theta$ im Vergleich zum zusätzlich erzielbaren Gewinn durch eine erhöhte Produktion zu hoch ist.

\section{Abbildung 9}

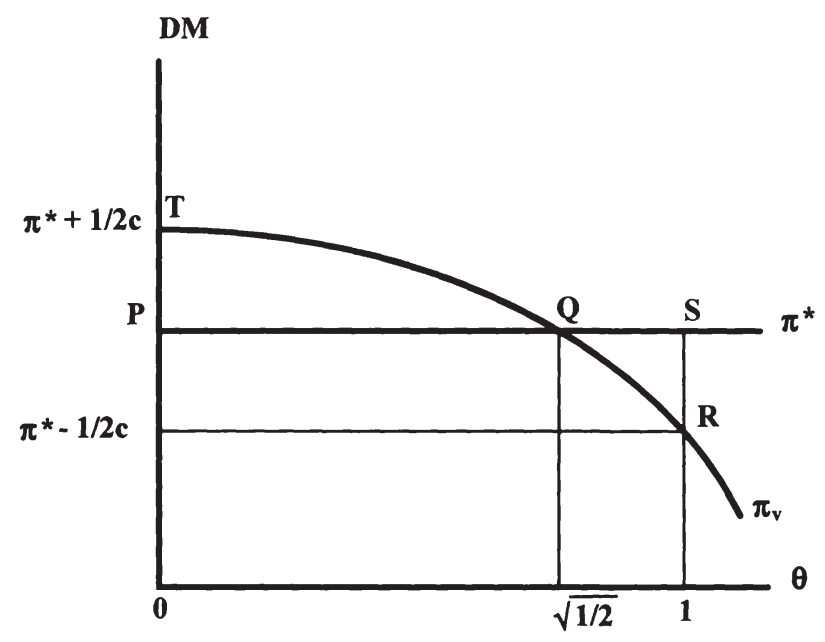

Mit diesem einfachen Beispiel sei die Analyse bilateraler Verhandlungen über externe Effekte abgeschlossen. Im nächsten Abschnitt sollen nun einige der bisher getroffenen Annahmen kritisch beleuchtet und mögliche Modellerweiterungen diskutiert werden.

\subsection{Diskussion der Modellannahmen}

Bei der Ableitung der Ergebnisse des vorhergehenden Abschnitts wurde eine Reihe von Annahmen getroffen. Im folgenden soll nun versucht werden zu klären, ob diese Annahmen ernsthafte Einschränkungen darstellen oder ob die Resultate auch unter anderen Rahmenbedingungen ihre Gültigkeit behalten. 


\subsubsection{Rechtfertigung des "Mechanism-Design"-Ansatzes}

Zunächst erscheint es angebracht, die Wahl der grundlegenden Modellstruktur zu rechtfertigen. Es gibt natürlich zahlreiche Möglichkeiten der Modellierung nichtkooperativer Verhandlungen bei unvollständiger Information. Da die Ergebnisse in starkem Maße von der konkreten Struktur des Verhandlungsprozesses abhängen, ${ }^{26}$ stellt sich die Frage, welche Prozesse im einzelnen analysiert werden sollen. Man kann dabei so vorgehen, daß man exogen vorgegebene Prozesse untersucht, von denen man glaubt, da $\beta$ sie wichtige real existierende Verhandlungsstrukturen adäquat beschreiben. Was die Literatur zum "Property-Rights"-Ansatz bei asymmetrischer Information betrifft, so findet sich bereits bei Arrow (1979) ein bilaterales Verhandlungsmodell, in dem die uninformierte Partei Take-it-or-leave-it-Offerten unterbreitet, die die informierte Partei nur akzeptieren oder ablehnen kann. In diesem Modell wird gezeigt, daß die ungleiche Informationsverteilung zwischen den Verhandlungspartnern mit einer gewissen Wahrscheinlichkeit zu ex post ineffizienten Lösungen führen kann. Samuelson (1985) behandelt noch andere einfache Verhandlungsmodelle und kommt zu ähnlichen Resultaten. Er zeigt auch, daß das Aus$\mathrm{ma} ß$ der Ineffizienz der Verhandlungsergebnisse ceteris paribus mit der Modellierung des Verhandlungsprozesses variiert. Daß unvollständige Information zu Ineffizienzen führt, bestätigt sich aber auch in diesen Modellen.

Im Vergleich zur Analyse gegebener Verhandlungsstrukturen weist der in dieser Arbeit verwendete Mechanism-Design-Ansatz (MDA) einen wichtigen Unterschied auf: In diesem Modellrahmen kann einer der Verhandlungspartner (der Prinzipal) neben Output- und Transferfunktion, die seine Nutzenposition direkt tangieren, auch die Regeln des Verhandlungsspiels bestimmen. Diese Spielregeln können außerdem in gewissen Grenzen offen bleiben: Aus dem Enthüllungsprinzip folgt zwar, daß der Prinzipal sich in seinem Optimierungskalkül auf die Menge derjenigen Kontrakte beschränken kann, die dem Agenten keinen Anreiz mehr geben, seine private Information zu verschleiern. Das heißt aber nicht, da $\beta$ er als konkreten Verhandlungsprozeß unbedingt einen direkten Enthüllungsmechanismus wählen muß, in dem der Agent aufgefordert ist, die Information unmittelbar und wahrheitsgemä $B$ zu offenbaren. Das Ergebnis, das durch den direkten Enthüllungsmechanismus implementiert wird, definiert für den Prinzipal lediglich die Obergrenze seiner Möglichkeiten. Es ist also denkbar, daß der Prinzipal dieses Ergebnis mit einer ganzen

${ }^{26} \mathrm{Vgl}$. Sutton, (1986, S. 709). 
Reihe anderer Prozeduren, z.B. mit einem mehrstufigen Kommunikationsprozeß, implementieren kann. Er muß bei der Auswahl des Verhandlungsmodus nur darauf achten, durch geschickte Spezifikation der Spielregeln sein persönliches Optimum zu erreichen.

So betrachtet hat der MDA im Hinblick auf die Klärung der Frage, ob private Verhandlungen über externe Effekte bei asymmetrischer Information effizient sind oder nicht, einen entscheidenden Vorteil: Er ist in gewisser Hinsicht allgemeiner als die Analyse vorgegebener Verhandlungssprozesse, weil er eine ganze Klasse möglicher Prozesse umfaßt. ${ }^{27}$ Die Abgrenzung dieser Klasse ist dabei nicht völlig willkürlich, da nur solche Verhandlungsprozeduren in Frage kommen, die geeignet sind, den gewinnmaximalen Kontrakt zu implementieren. Man kann sagen, daß im Rahmen des MDA der Verhandlungsproze $B$ endogenisiert wird, weil er selbst Bestandteil des Optimierungsproblems ist. Gleichzeitig können aber die Entscheidungsvariablen weitgehend losgelöst vom Verhandlungsprozeß bestimmt werden. Der MDA ist in diesem Sinne stärker ergebnisorientiert, weil er den eigentlichen Verhandlungsproze $B$ ausblendet und sich auf ein bestimmtes Ergebnis konzentriert, das mittels unterschiedlicher Prozesse erreicht werden könnte. Dies ist insofern vorteilhaft, als man bei der Beurteilung der Effizienzeigenschaften von Verhandlungen weniger am Verhandlungsablauf selbst, als am realisierten Allokationsergebnis interessiert ist. Dieses Allokationsergebnis muß aber, wie in Abschnitt 2.4 gezeigt wurde, zwangsläufig suboptimal sein, wenn die uninformierte Partei, in unserem Fall also der Verursacher der Externalität, offeriert.

\subsubsection{Die Rolle der Verhandlungsmacht}

Die Fragestellung, die an diese Überlegungen natürlicherweise anknüpft, lautet nun: Ändern sich die Ergebnisse, wenn der Geschädigte die Verhandlungsmacht besitzt und sich in der Rolle des Offerierenden befindet. Wenn man die Informationsstruktur unverändert läßt und weiterhin annimmt, daß die Gewinnfunktion des Schädigers allgemein bekannt ist, dann ist die Antwort auf diese Frage klar: Der Geschädigte kann den Schädiger immer auf dem Reservationsnutzenniveau halten, indem er ihm nur solche Verträge anbietet, die diesen dazu verpflichten, ihm selbst den gesamten Produktionsgewinn zu überlassen. Was die Wahl des Produktionsni-

${ }^{27} \mathrm{Vgl}$. dazu auch Binmore, Osborne und Rubinstein (1992, S. $217 \mathrm{ff}$.). 
veaus betrifft, so hat der Geschädigte dann natürlich keinen Anreiz, Angebote zu unterbreiten, die seinen wahren Schadenstyp verschleiern. Er wird also dem Schädiger vorschlagen, die effiziente Menge $x^{*}(\theta)$ zu produzieren, die die Differenz zwischen Produktionsgewinn und erlittenem Schaden maximiert. ${ }^{28}$

Man könnte nun angesichts dieser Möglichkeit auf den Gedanken kommen, nach Wegen zu suchen, durch eine Art "Umverteilung von Verhandlungsmacht" zu einer effizienten Lösung zu gelangen. Dagegen ist einzuwenden, daß Verhandlungsmacht, anders als Eigentumsrechte, nicht einfach (etwa durch den Staat) einer beliebigen Partei zugewiesen werden kann. Verhandlungsmacht drückt sich in dem hier verwendeten Modell letztendlich darin aus, daß eine Partei den ersten Spielzug machen kann. Welche Partei dies sein wird, dürfte jedenfalls von Faktoren abhängen, auf die eine zentrale Behörde kaum Einfluß hat. In der Praxis wird es wohl die Partei sein, die vielleicht aufgrund eines Informationsvorsprungs als erste auf das bestehende Externalitätsproblem aufmerksam wird und klug genug ist, den "firstmover-advantage" zu nützen. Dabei ist es natürlich auch erforderlich, daß sie über Möglichkeiten der Selbstbindung verfügt, die nötig sind, um Take-it-or-leave-itOfferten durchsetzen zu können.

Abgesehen davon ist es auch unplausibel anzunehmen, der Geschädigte sei über die Gewinnfunktion des Schädigers vollständig informiert. Realistischer ist es, von einer Situation auszugehen, in der beide Verhandlungspartner private Information über entscheidungsrelevante Charakteristika haben. Wenn man diese Annahme trifft, hat man ein sog. "Mechanism-Design-Problem mit informiertem Prinzipal". Dieses Problem tritt dann auf, wenn neben dem Agenten auch der Prinzipal über private Information verfügt. Es findet sich in der Literatur zum erstenmal bei Myerson (1983), allerdings im Rahmen der kooperativen Spieltheorie. In bezug auf nichtkooperative Modelle sind zwei Arbeiten von Maskin und Tirole (1990 und 1992) von Bedeutung, die jeweils eine von zwei möglichen Arten privater Information behandeln: Die neuere Arbeit (1992) behandelt den Fall, in dem die private Information des Prinzipals direkt als Parameter in die Nutzenfunktion des Agenten eingeht ("common values"). Als Beispiel hierfür könnte man ein Modell anführen, in dem ein Monopolist einem Nachfrager, dessen Zahlungsbereitschaft er nicht kennt, ein Gut von gegebener Qualität anbietet, wobei der Nachfrager die Qualität nicht beobachten kann. Die Qualität des Gutes muß natürlich sinnvollerweise als Parameter in die Nutzenfunktion des Käufers eingehen.

${ }^{28} \mathrm{Vgl}$. auch Schweizer (1988, S. 258 f.) und Illing (1992, S. 567). 
In der älteren Arbeit (1990) wird der Fall analysiert, in dem die Information des Prinzipals nur indirekt über die Abhängigkeit der Entscheidungsvariablen von seinen typischen Merkmalen in die Präferenzen des Agenten eingeht ("private values"). Dieser Fall ist für das hier betrachtete Szenario relevant. So hängt beispielsweise der Gewinn des Schädigers bei gegebener Produktionsmenge und gegebener Transferzahlung sicher nicht davon ab, welcher Schadenstyp beim Geschädigten vorliegt. Ebenso ist der Nutzen des Geschädigten ceteris paribus unabhängig von der konkreten Gestalt der Gewinnfunktion des Schädigers.

Die Frage ist nun, ob die Existenz privater Information auf Seiten des offerierenden Verhandlungspartners die Resultate aus Abschnitt 2.4 entscheidend verändert. Diese Möglichkeit kommt deshalb in Betracht, weil der vom Prinzipal angebotene Kontrakt von dessen typischen Merkmalen abhängt und der Agent aus der bloBen Offerte Rückschlüsse auf den Typ des Prinzipals ziehen könnte. Daraus könnte dem Prinzipal ein Anreiz erwachsen, den Mechanismus so zu konzipieren, daß sein wahrer Typ zunächst verschleiert wird. Die Grundidee dabei ist folgende: Der Prinzipal muß bei seiner Entscheidung über den vorzuschlagenden Kontrakt die Bedingungen der Anreizverträglichkeit und der individuellen Rationalität in bezug auf den Agenten berücksichtigen. Wenn der Agent den Informationsparameter des Prinzipals kennt, so müssen diese Nebenbedingungen genau für diesen Parameter erfüllt sein. Hat der Agent dagegen nur unvollständige Information über den wahren Typ des Prinzipals, so müssen (AV) und (IR) nur im Durchschnitt über alle möglichen Informationsparameter des Prinzipals erfült sein. Die Unsicherheit des Agenten bewirkt demnach im Optimierungskalkül des Prinzipals eine Lockerung der Nebenbedingungen, so da $\beta$ dieser sich jedenfalls nicht verschlechtern kann, wenn er seine private Information nicht sofort preisgibt.

Maskin und Tirole (1990) zeigen nun, daß sich der Prinzipal im allgemeinen strikt besserstellt, wenn er diese Unsicherheit zu Beginn des Spiels aufrechterhält. ${ }^{29}$ Es wird aber auch gezeigt, daß dieses Resultat nicht mehr gilt, wenn die Präferenzen sowohl des Prinzipals als auch des Agenten quasilinear sind. In diesem Fall ist es gleichgültig, ob der Prinzipal seinen Typ sofort offenbart oder nicht. Das bedeutet, da $B$ in einem Modell mit zweiseitig asymmetrischer Information und quasilinearen Präferenzen die private Information des Prinzipals keine Rolle spielt. Alles ist dann wie in einem Modell mit einseitig asymmetrischer Information über die Merkmale des Agenten.

${ }^{29}$ Diese Aussage gilt nur im Fall von "private values" (Siehe Maskin und Tirole (1990, S. 382)). 
Wenn man dieses Ergebnis auf die hier gewählte Modellspezifikation (mit quasilinearen Präferenzen!) anwendet, kommt man zu folgendem Schluß: Fälle mit zweiseitig asymmetrischer Information müssen nicht gesondert untersucht werden; es genügt eine Analyse der Szenarien mit einseitig asymmetrischer Information.

Der Fall, in dem der Schädiger offeriert, wurde in Abschnitt 2.4 ausführlich behandelt. Würde man nun dem Geschädigten das Recht des ersten Zuges einräumen und annehmen, die Gewinnfunktion des Schädigers sei unbekannt, wäre die Analyse analog zu der in 2.4, nur mit vertauschten Rollen. Der Geschädigte hätte jetzt das Informationsproblem und müßte, um dieses zu lösen, dem Schädiger eine Informationsrente zugestehen, so daß natürlich auch hier keine effiziente Verhandlungslösung zustande kommen wird. Insofern sind die Resultate des oben explizit behandelten Modells qualitativ auf eine ganze Reihe weiterer Szenarien übertragbar.

Es muß aber nochmals ausdrücklich darauf hingewiesen werden, daß diese Argumentation nur unter der Annahme quasilinearer Präferenzen gültig ist. Diese Voraussetzung ist natürlich sehr restriktiv. Dennoch stellt sie eine der Standardannahmen in der einschlägigen Literatur dar. Bis zur Charakterisierung anreizkompatibler und individuell rationaler Kontrakte kommt man auch ohne sie aus, ${ }^{30}$ bei der Lösung des Optimierungskalküls des Prinzipals bringt sie jedoch eine deutliche Erleichterung mit sich. Welche inhaltlichen Auswirkungen ein Verzicht auf diese Annahme haben könnte, kann im Rahmen dieser Arbeit nicht geklärt werden. Die abgeleiteten Resultate gelten aber zumindest für den Fall, daß es sich bei Schädiger und Geschädigtem um Unternehmen handelt, da unter diesen Umständen Einkommenseffekte keine Rolle spielen.

\subsubsection{Die Rolle der Eigentumsordnung}

Im nächsten Schritt wird nun untersucht, inwieweit die Ausgestaltung der Eigentumsordnung für die Effizienzeigenschaften der Verhandlungslösung ausschlaggebend ist. Bisher wurde angenommen, daß das Verursacherprinzip gilt, d.h. der Schädiger darf die Produktion des Gutes x nicht aufnehmen, wenn der Geschädigte nicht damit einverstanden ist. Ersterer befindet sich damit in der Rolle des Käufers, letzterer in der Rolle des Verkäufers des Rechts auf die Ausübung des externen Effekts. Man könnte nun die gleichen Analysen wie in Abschnitt 2.4 für den ande-

$30 \mathrm{Vgl}$. Fudenberg und Tirole (1991, S. 257ff.). 
ren Extremfall der Verteilung der Eigentumsrechte, das Laisser-Faire-Prinzip, durchführen. Anstelle einer expliziten Behandlung dieses Falles sei jedoch auf zwei neuere Literaturbeiträge verwiesen. Demougin und Illing (1991) charakterisieren die Verhandlungslösung bei Zugrundelegung des Laisser-Faire-Prinzips für den Fall einer stetigen Verteilung der Grenzschäden $\theta$ in einem abgeschlossenen Intervall. In Illing (1992) wird der einfachere Fall mit nur zwei möglichen Ausprägungen des Grenzschadens analysiert. Wie nicht anders zu erwarten, ist das Verhandlungsergebnis auch unter diesen veränderten Rahmenbedingungen ineffizient. Die ökonomische Intuition für dieses Resultat liegt auf der Hand: Gilt das Laisser-Faire-Prinzip, so darf der Schädiger seine Produktion nach Belieben ausdehnen. Der Geschädigte muß sich dann eine gewünschte Reduktion des Outputs erkaufen. Wenn nun der Schädiger wieder das Angebot unterbreiten und den Schadenstyp des Geschädigten beobachten könnte, würde er ihm eine Produktionseinschränkung gegen ein "Bestechungsgeld" in Höhe der vermiedenen Schäden anbieten. Die Verhandlungslösung wäre effizient, da der Schädiger die Produktion bis auf dasjenige Niveau begrenzen würde, bei dem die zusätzlichen Einnahmen aus Bestechungsgeldern, die dem vermiedenen Grenzschaden entsprechen, mit seinen marginalen Gewinneinbußen übereinstimmen. Wenn der Schädiger den Grenzschaden dagegen nicht beobachten kann, dann hat ein Geschädigter mit hohem Schadenstyp einen Anreiz, sich als Typ mit niedrigem Grenzschaden auszugeben, da die Kosteneinsparungen und damit die an den Schädiger zu leistenden Zahlungen umso niedriger sind, je niedriger der Grenzschaden ist. Bei einer Untertreibung muß sich der Geschädigte zwar mit einer geringeren Produktionseinschränkung zufriedengeben, dafür bezahlt er aber pro vermiedene Mengeneinheit weniger, als es seiner tatsächlichen Zahlungsbereitschaft entspräche. Der Schädiger muß also in diesem Fall Typen mit hohen Schäden eine Informationsrente gewähren, wenn er eine wahrheitsgemäße Offenbarung dieser Schäden erreichen will. Diese Informationsrente kann er aber dadurch schmälern, daß er Geschädigten mit niedrigeren Grenzschäden eine geringere als die paretooptimale Produktionseinschränkung anbietet. Die Verhandlungslösung wird also auch bei Zugrundelegung des Laisser-Faire-Prinzips i.d.R. ineffizient sein. Während aber im Fall des Verursacherprinzips die Produktionsmengen mit Ausnahme derjenigen für den $\theta^{-}$-Typ zu niedrig sind, sind sie nun bis auf die Menge für $\operatorname{den} \theta^{+}$-Typ zu hoch.

Auf der Grundlage der Resultate von Abschnitt 2.4 und der ergänzenden Überlegungen dieses Abschnitts gelangt man zu dem Schluß, daß man von bilatera- 
len Internalisierungsverhandlungen unter den Bedingungen unvollständiger Information keine ex post effizienten Allokationsergebnisse erwarten kann. Der Grund für die Ineffizienz liegt darin, daß die offerierende Partei ihrem Verhandlungspartner eine Informationsrente zu zahlen hat, um ihn zur Preisgabe seiner privaten Information zu bewegen. Dadurch wird ein "Keil" zwischen die einzel- und gesamtwirtschaftlichen Grenzkosten einer Ausdehnung (im Fall des Verursacherprinzips) bzw. einer Einschränkung (im Fall des Laisser-Faire-Prinzips) des Externalitätsniveaus getrieben, so daß dieses je nach Ausgestaltung der Eigentumsordnung im Vergleich zum First-Best-Niveau zu niedrig bzw. zu hoch ist. Immerhin können aber trotz unvollständiger Information partielle Wohlfahrtsgewinne erzielt werden. Wie gut oder wie schlecht die Verhandlungslösung unter Effizienzgesichtspunkten wirklich ist, kann erst beurteilt werden, wenn man sie im Vergleich mit zentralen Internalisierungsmaßnahmen betrachtet. Es muß erst überprüft werden, ob eine Zentralinstanz besser mit dem Informationsproblem fertig wird und höhere Wohlfahrtsgewinne erzielen kann. Dieser Frage wird aber erst später im zweiten Teil der Arbeit nachgegangen. 


\section{Internalisierungsverhandlungen mit mehreren Beteiligten}

\subsection{Problemstellung und Begründung der Modellwahl}

Wie wir im vorangegangenen Kapitel gesehen haben, kann man bereits im einfachsten Fall bilateraler Verhandlungen über externe Effekte nicht erwarten, zu effizienten Allokationen zu gelangen. Dieses Ergebnis wurde in einem Modellrahmen, in dem das Verursacherprinzip galt und der Schädiger über die gesamte Verhandlungsmacht verfügte, abgeleitet. Der Grund für dieses Resultat lag darin, daß der Geschädigte, der im Gegensatz zum Schädiger über die konkrete Ausprägung seines Schadenskoeffizienten voll informiert war, einen Anreiz hatte, seine private Information zu verschleiern, um seine Nutzenposition zu erhöhen. Der Schädiger war deshalb gezwungen, dem Geschädigten eine Informationsrente zuzugestehen, um ihm diesen Anreiz zu nehmen. Diese Notwendigkeit führte mit Ausnahme des Falles, in dem der Geschädigte den niedrigsten aller möglichen Schadensparameter aufwies, zu einer Divergenz zwischen den Grenzkosten einer Produktionserhöhung, die der Schädiger bei der Maximierung seines Gewinns zugrundelegte, und den gesamtwirtschaftlichen Grenzkosten, so daß sich zwangsläufig pareto-inferiore Verhandlungsergebnisse einstellten. In Abschnitt 2.6 wurde begründet, warum auch unter allgemeineren Bedingungen, insbesondere bei anderen Konstellationen von Eigentumsrecht und Verhandlungsmacht, mit Ineffizienzen gerechnet werden muß. Die qualitativen Ergebnisse des explizit analysierten Modells können somit weitgehend als allgemeingültig bezeichnet werden.

Man kann sich nun fragen, ob sich ein anderes Bild ergibt, wenn man die Anzahl der Verhandlungsteilnehmer auf einer oder auf beiden Seiten erhöht. Die Vermutung, daß in solchen Fällen bessere Allokationsergebnisse erreicht werden könnten, erscheint auf den ersten Blick nicht unbegründet, wenn man einer ökonomischen Standardargumentation folgt: Bei einer großen Anzahl von Marktteilnehmern wird der Einfluß des einzelnen auf den Preis für das Gut, das auf dem betrachteten Markt gehandelt wird, vernachlässigbar gering. Folglich besteht für die Individuen kein Anreiz mehr, die Tauschbedingungen durch eine falsche Angabe ihrer subjektiven Wertschätzungen zu manipulieren, so da $B$ man effiziente Allokationsergebnisse erwarten kann.

In der Tat findet man in der Literatur Beiträge, die derartige Überlegungen zu bestätigen scheinen. So zeigt beispielsweise Wilson (1985), daß im Rahmen eines 
speziellen Auktionstyps, einer sog. "double auction"1, trotz unvollständiger Information über die Wertschätzungen, die die Auktionsteilnehmer den zu versteigernden Objekten beimessen, eine effiziente Allokation erreicht wird, wenn die Anzahl der Anbieter und Nachfrager gegen unendlich geht. In einem Modell mit ähnlicher Marktstruktur zeigen Gresik und Satterthwaite (1989), daß für eine bestimmte Klasse von Tauschmechanismen die aufgrund unvollständiger Information zu erwartenden Wohlfahrtsverluste im Verhältnis zu den erwarteten Tauschgewinnen bei hypothetischer Realisation ex post effizienter Allokationen gegen Null konvergieren, wenn die Anzahl der Tauschpartner unendlich groß wird.

Die Frage ist nun, inwieweit sich diese Resultate auf das hier untersuchte Externalitätsproblem übertragen lassen. Dies ist nur dann möglich, wenn Schädiger und Geschädigte miteinander in Tauschbeziehungen stehen, die mit den in den o.g. Arbeiten unterstellten vergleichbar sind. Abgesehen davon, daß bei Wilson und Gresik und Satterthwaite unteilbare Güter gehandelt werden, ist es insbesondere erforderlich, daß die Externalität die Eigenschaften privater Güter, also "Ausschließbarkeit" und "Rivalität im Konsum" aufweist. Diesbezüglich sind aber Bedenken angebracht, da in der Realität die meisten Arten von externen Effekten typischerweise den Charakter eines öffentlichen Gutes haben. So nimmt beispielsweise die individuelle Schadstoffbelastung der Anlieger eines Kohlekraftwerks nicht dadurch ab, daß die Anzahl der Geschädigten (etwa durch Zuzüge) steigt; oder Lärmbelästigung wird durch eine erhöhte Anzahl von "Mithörern" für den einzelnen nicht erträglicher. Andererseits würde jeder Geschädigte davon profitieren, wenn es einem seiner Leidensgenossen gelänge, den Urheber der jeweiligen Beeinträchtigung mittels einer Bestechungszahlung zu einer Einschränkung seiner Aktivität zu bewegen. Die Liste der Beispiele könnte beliebig fortgesetzt werden. Festzuhalten

\footnotetext{
${ }^{1}$ Unter einer "double auction" versteht man einen speziellen Tauschmechanismus zwischen einer best. Anzahl von potentiellen Verkäufern, die je eine Einheit eines best. Gutes verkaufen wollen, und einer Gruppe potentieller Käufer, die je eine Einheit dieses Gutes erwerben wollen. Die Allokation der angebotenen Güter erfolgt im Rahmen einer Versteigerung, bei der die Käufer einem Auktionator mitteilen, welchen Preis sie für das gewünschte Objekt zu zahlen bereit sind. Die Verkäufer geben ihrerseits an, zu welchem Preis sie verkaufen wollen. Der Auktionator fixiert auf der Basis dieser Gebote und Forderungen einen Preis, bei dem der Markt gräumt ist. Käufer, deren Gebot größer ist als dieser Preis, können kaufen, Verkäufer, deren Forderung kleiner ist, können verkaufen. Falls die Gebote bzw. Forderungen einiger Käufer bzw. Verkäufer mit dem Gleichgewichtspreis übereinstimmen, richtet sich das Transaktionsvolumen nach der "kürzeren" Marktseite. Dieser Auktionstyp ist u.a. deshalb interessant, weil er sich gut zur Modellierung des Walrasianischen Preisbildungsmechanismus eignet. (Vgl. Wilson (1985, S. 1101)).
} 
bleibt, daß es im Hinblick auf real existierende Externalitätsprobleme sinnvoll erscheint, deren Öffentlichen-Guts-Charakter bei der Modellierung von Verhandlungen mit mehreren Teilnehmern zu berücksichtigen. Damit ist klar, daß die Resultate von Wilson bzw. Gresik und Satterthwaite nicht ohne weiteres übernommen werden können, sondern in einem problemgerechten Modellrahmen erst überprüft werden müssen.

Dabei stellt sich aber ein Problem, das bereits in der Einführung angesprochen wurde: Aufgrund des Öffentlichen-Guts-Charakters externer Effekte kann es bei privaten Verhandlungen unter Umständen zu Free-Rider-Verhalten der beteiligten Individuen kommen, was eine Durchsetzung von First-Best-Allokationen selbst bei vollständiger Information zweifelhaft erscheinen läßt. Ob Free-Riding auftritt oder nicht hängt in entscheidendem Maße von der jeweiligen Kombination von Eigentumsrecht und Verhandlungsmacht ab. Wenn man beispielsweise annimmt, es gelte das Laisser-Faire-Prinzip, und eine Gruppe von Geschädigten müsse einem oder mehreren Schädigern Kompensationszahlungen für Produktionseinschränkungen anbieten, so ist zu erwarten, daß die ausgehandelte Produktionsmenge auch bei vollständiger Information zu hoch sein wird: Wenn man davon ausgeht, daß die Geschädigten ihre Beiträge zu den Kompensationszahlungen ohne bindende Absprachen isoliert voneinander bestimmen müssen, dann hat jeder Geschädigte aufgrund der Öffentlichen-Guts-Eigenschaft einer Produktionseinschränkung einen Anreiz, sich mit seinen eigenen Beiträgen auf Kosten der anderen Gruppenmitglieder zurückzuhalten. Es wird also zu einer für diese Situation typischen Unterversorgung mit dem öffentlichen Gut "Produktionseinschränkung" kommen.

Dieses Beispiel soll zeigen, daß bei der Wahl des Modells die Konstellation von Eigentumsrecht und Verhandlungsmacht so festzulegen ist, daß die Effekte, die vom Informationsproblem ausgehen, nicht von Trittbrettfahrerproblemen, die schon bei vollständiger Information auftreten, überlagert werden. Dies kann beispielsweise dadurch vermieden werden, daß man annimmt, es gebe nur einen Schädiger und mehrere Geschädigte, wobei das Verursacherprinzip gelten und der Schädiger die gesamte Verhandlungsmacht besitzen soll. Trittbrettfahrerverhalten im oben beschriebenen Sinn kann in dieser Modellversion nicht auftreten, da der Schädiger jedem einzelnen Geschädigten eine Zahlung anbieten kann, ohne daß diese sich in der Gruppe gemeinsam auf eine bestimmte Summe einigen müssen. Wenn der Schädiger vollständige Information über die Schadenskoeffizienten der Geschädigten hätte, würde er jedem den Ersatz der tatsächlichen Schäden anbieten, da dies 
ausreichen würde, um die Akzeptanz seiner Vertragsangebote zu sichern. Wenn man wieder davon ausgeht, daß der Schaden, der bei einem einzelnen Individuum verursacht wird, durch die Schadensfunktion $\mathrm{d}_{\mathrm{i}}(\mathrm{x})=\theta_{\mathrm{i}} \mathrm{x}$ beschrieben werden kann, dann hätte der Schädiger Kosten in Höhe von $x \sum_{i=1}^{n} \theta_{i}$ zu tragen und könnte einen Gewinn in Höhe von $b(x)-x \sum_{i=1}^{n} \theta_{i}$ erzielen. Dieser Gewinn ist dann maximal, wenn der Schädiger diejenige Produktionsmenge wählt, bei der der marginale Bruttogewinn mit der Summe der Grenzschäden über alle Geschädigten übereinstimmt. Die Optimallösung für den Schädiger bei vollkommener Information, $x^{*}\left(\theta_{1}, \ldots, \theta_{n}\right)$, resultiert also aus der individuellen Optimalitätsbedingung $b^{\prime}\left(x^{*}\left(\theta_{1}, \ldots, \theta_{n}\right)\right)$ $=\sum_{i=1}^{n} \theta_{i}$. Dies ist natürlich auch die Bedingung für ein gesamtwirtschaftlich optimales Produktionsniveau, so daß bei Verhandlungen unter vollständiger Information in diesem Szenario keine Wohlfahrtsverluste auftreten.

Die eben beschriebene Variante stellt nur eine Möglichkeit der Modellierung privater Verhandlungen mit mehreren Beteiligten dar. Neben der Tatsache, daß bei vollständiger Information eine pareto-optimale Verhandlungslösung realisiert wird und somit diejenigen Wohlfahrtswirkungen, die auf unvollständige Information zurückzuführen sind, isoliert betrachtet werden können, erscheint die Analyse dieses Szenarios noch aus zwei weiteren Gründen besonders attraktiv: Zum einen besitzt es einiges an empirischer Relevanz, wenn man nur an die Umweltverschmutzung durch die Großindustrie denkt. In diesem Fall steht meistens ein Schädiger vielen Geschädigten gegenüber. Was die Eigentumsordnung betrifft, so ist zumindest für die Bundesrepublik Deutschland vom Verursacherprinzip auszugehen, da allgemeine Rechtsvorschriften den Schädiger zum Schadensersatz verpflichten, sofern der Geschädigte eine Beeinträchtigung nachweisen kann. ${ }^{2}$ Zum anderen knüpft dieses Szenario unmittelbar an das Modell des zweiten Kapitels an, so daß die nötigen Erweiterungen ohne allzu großen Aufwand vorgenommen werden können.

Das in diesem Kapitel dargestellte Modell geht im wesentlichen auf eine Arbeit von Raffael Rob (1989) zurück. Die Zielsetzung besteht zunächst darin, das Modell aus Kapitel 2 auf einen Rahmen mit mehreren Geschädigten zu erweitern und Bedingungen für eine gewinnmaximale Vertragsgestaltung herauszuarbeiten. Dies wird der Gegenstand von Abschnitt 3.2 sein. In Abschnitt 3.3 wird gezeigt, daß

${ }^{2}$ Vgl. z.B. $§ 823$ Abs. I BGB. Die Tatsache, daß der Geschädigte die Beweislast hat, dürfte aber sehr häufig einen Schadensersatz vereiteln. 
die von Rob gewählte Modellspezifikation als Spezialfall aus dem vorher entwickelten Modell hervorgeht. Aus Vereinfachungsgründen werden die weiteren Analysen dann auf der Basis dieses Spezialfalls durchgeführt. Zuerst werden die wichtigsten Ergebnisse des Rob-Modells für den Fall mit zwei Geschädigten präsentiert. Die Präsentation wird in Anbetracht der relativ komplexen Zusammenhänge durch eine speziell entwickelte graphische Darstellungsmethode unterstützt. Dabei wird auch auf das in Kapitel 2 angesprochene Problem multipler Gleichgewichte in PrinzipalAgenten-Problemen mit mehreren Agenten eingegangen. Anschließend werden zwei alternative Maßgrößen vorgestellt, die eine Beurteilung der Effizienzeigenschaften der Verhandlungslösung ermöglichen. Für eines dieser Wohlfahrtsmaße wird gezeigt, daß sich keineswegs pareto-optimale Lösungen durchsetzen, wenn die Anzahl der Geschädigten gegen Unendlich geht. Es wird sich vielmehr herausstellen, daß sich die Effizienzeigenschaften der Verhandlungslösung mit steigender Zahl der Geschädigten verschlechtern.

\subsection{Erweiterung des Verhandlungsmodells auf mehrere Geschädigte}

\subsubsection{Die Informationsstruktur}

$\mathrm{Zu}$ Beginn der allgemeinen Modellbeschreibung ist es nötig, die grundlegende Informationsstruktur zwischen Schädiger und Geschädigten zu beschreiben. Wie in Kapitel 2 wird angenommen, daß der Schädiger die individuellen Schadensparameter $\theta_{\mathrm{j}}$ der $\mathrm{j}=1 \ldots$... Geschädigten nicht beobachten kann. Er weiß nur, daß $\theta_{\mathrm{j}}$ im Intervall $\mathrm{T}_{\mathrm{j}}=\left[\theta_{\mathrm{j}}{ }^{-}, \theta_{\mathrm{j}}^{+}\right]$liegen kann und mit der Wahrscheinlichkeitsdichte $\mathrm{f}_{\mathrm{j}}\left(\theta_{\mathrm{j}}\right)$ stochastisch unabhängig in $\mathrm{T}_{\mathrm{j}}$ verteilt ist. Die zugehörige Wahrscheinlichkeitsfunktion wird mit $\mathrm{F}_{\mathrm{j}}\left(\theta_{\mathrm{j}}\right)$ bezeichnet. Was den Informationsstand der Geschädigten betrifft, sind prinzipiell zwei Möglichkeiten denkbar: Man nimmt entweder an, sie kennen gegenseitig ihre Schadensparameter oder geht davon aus, da $\beta$ jeder nur sein eigenes $\theta_{\mathrm{j}}$ kennt und über die Schadenskoeffizienten der anderen die gleiche Information besitzt wie der Schädiger. Im folgenden wird mit der letzten Variante gearbeitet, da es nicht sehr plausibel erscheint, daß die Geschädigten über die $\theta_{j}$ ihrer Opponenten besser informiert sind als der Schädiger. In der Terminologie der Informationsökonomie stellen also die $\theta_{j}$ "private Information" dar, während die $T_{j}$ 
und die $\mathrm{f}_{\mathrm{j}}($.$) "common knowledge" sind. { }^{3}$ Die Annahme, daß die $\mathrm{f}_{\mathrm{j}}$ common knowledge sind, trägt wesentlich zur Vereinfachung der Analyse bei. Ein Verzicht auf diese Annahme hätte folgende Konsequenz: Man müßte unterstellen, daß Schädiger und Geschädigte von einer subjektiven Vermutung über die Wahrscheinlichkeitsverteilung von $\theta_{\mathrm{j}}$ in $\mathrm{T}_{\mathrm{j}}$ ausgehen. Man müßte dann für jedes der $\mathrm{i}=0$...n Individuen mit einer unterschiedlichen Wahrscheinlichkeitsdichte $f_{j}\left(\theta_{j} / i\right)$ arbeiten, die diese subjektiven Vermutungen beschreibt. ${ }^{4}$ Wenn man es sich dann wieder einfach macht, nimmt man an, die $\mathrm{f}_{\mathrm{j}}\left(\theta_{\mathrm{j}} / \mathrm{i}\right)$ seien common knowledge. Eigentlich müßte man aber wieder für jedes Individuum dessen Vermutungen über die Vermutungen der anderen beschreiben usw. Dieser unendliche Regreß wird durch die Annahme objektiver und allgemein bekannter $f_{j}\left(\theta_{j}\right)$ gleich von vornherein unterbrochen.

Zur Vereinfachung der Notation sollen die folgenden Definitionen gelten:

(3.1) $T:=X_{j}^{n} T_{j}$ (n-faches kartes. Produkt der $T_{j}, j=1 \ldots n$ ),

(3.2) $T_{-j}:=X_{j \neq i}^{n} T_{j}$,

(3.3) $\theta:=\left(\theta_{1}, \ldots, \theta_{n}\right)$,

(3.4) $\theta_{-i}:=\left(\theta_{1}, \ldots, \theta_{i-1}, \theta_{i+1}, \ldots, \theta_{n}\right)$,

(3.5) $f(\theta):=\prod_{j=1}^{n} f_{j}\left(\theta_{j}\right)$ (wegen stochastischer Unabhängigkeit der $\theta_{j}$ ),

$$
f_{-i}\left(\theta_{-i}\right):=\prod_{j=1, j \neq i}^{n} f_{j}\left(\theta_{j}\right)
$$

\subsubsection{Bayesianische Gleichgewichte und Enthüllungsprinzip}

Das Problem des Schädigers besteht nun wieder darin, angesichts der oben beschriebenen Informationsstruktur einen Mechanismus zu konzipieren, der seinen erwarteten Gewinn maximiert. Dazu muß er wieder die Spielstruktur, eine Output-

\footnotetext{
${ }^{3}$ Eine Information gilt als "common knowledge", wenn jedes Individuum über diese Information verfügt und jeder weiß, daß jeder darüber verfügt und jeder weiß, daß jeder dies weiß usw.

${ }^{4}$ Der Index 0 steht für den Schädiger.
} 
funktion $\mathrm{x}$ und für jeden der $\mathrm{n}$ Geschädigten eine Transferfunktion $\mathrm{z}_{\mathrm{j}}$ bestimmen. Dabei hat er darauf zu achten, daß eine Teilnahme am Spiel für alle Geschädigten individuell rational ist.

Im Unterschied zum Fall mit einem Geschädigten handelt es sich bei diesem Spiel aber nicht mehr nur um eines zwischen Prinzipal und Agent, sondern man hat zusätzlich ein Teilspiel zwischen den einzelnen Agenten. In diesem Teilspiel verfügt jeder Agent über eine bestimmte Menge $S_{j}$ wählbarer Signale, aus der er dem Prinzipal ein Signal $s_{j}$ übermitteln muß. Der Prinzipal wählt auf der Grundlage dieser Signale einen Output $x\left(s_{1}, \ldots, s_{n}\right)$ und Transferzahlungen $z_{j}\left(s_{1}, \ldots, s_{n}\right)$, wobei die Agenten wieder wissen, wie der Prinzipal ihre Signale in den Funktionen $x($.$) und z_{j}($. verarbeitet.

Welches Signal der einzelne Agent dem Prinzipal übermittelt, hängt i. a. davon ab, welche Signale die übrigen Agenten senden. Um selbst zu einer optimalen Entscheidung über das zu sendende Signal zu gelangen, muß ein Spieler das Verhalten seiner Gegenspieler prognostizieren und sein eigenes Signal als beste Antwort auf diese Prognose ermitteln. Dies wird durch die Annahme, jeder Geschädigte kenne nur seinen eigenen Schadensparameter $\theta_{\mathrm{j}}$, erschwert, da die Spieler in diesem Fall die Nutzenfunktionen $v_{i}\left(x, z, \theta_{i}\right)$ ihrer Gegenspieler nicht kennen. Die Spielsituation, in der sich die Geschädigten befinden, ist eine mit unvollständiger Information. Ein Spiel mit unvollständiger Information liegt immer dann vor, wenn einer oder mehrere Spieler als unterschiedliche "Typen" vorkommen können und a priori nicht klar ist, welcher Typ jeweils vorliegt. ${ }^{5}$

Solche Spiele galten lange Zeit als unlösbar, denn, so wurde argumentiert, wenn die Spieler nicht wüßten, mit welchen Typen von Gegenspielern sie es zu tun haben, seien ihnen die "Regeln" des Spiels nicht bekannt, weil sie weder die Auszahlungen der anderen Spieler noch ihre eigenen Pay-Offs kennen würden. Sie wüßten also gar nicht, welches Spiel sie überhaupt spielen. Dies sei aber Voraussetzung für die Lösbarkeit. ${ }^{6}$ Erst durch die Beiträge von Harsanyi (1967/1968) wurde die Analyse solcher Spiele ermöglicht. Harsanyi führte zu Beginn des Spiels einen Zug der "Natur" als zusätzlichen Spieler ein, durch den die Typen der Spieler per Zufallsauswahl festgelegt werden und machte dadurch aus einem Spiel mit

\footnotetext{
${ }^{5}$ Zur genauen Definition des Begriffs "unvollständige Information" und zu seiner systematischen Abgrenzung gegenüber den Begriffen "unvollkommene Information", "asymmetrische Information" und "Unsicherheit" vgl. Rasmusen (1989, S. 51 ff.).

${ }^{6} \mathrm{Vgl} \mathrm{z.B.} \mathrm{Rasmusen} \mathrm{(1989,} \mathrm{S.} \mathrm{55).}$
} 
"unvollständiger Information" über die Spielertypen eines mit "unvollkommener Information" über den Zug der Natur. In diesem Spiel sind die Regeln wieder allen Spielern bekannt. ${ }^{7}$

Das grundlegende Konzept zur Lösung von Spielen mit unvollständiger Information ist das des Bayesianischen Gleichgewichts. Dieses Gleichgewichtskonzept soll im folgenden kurz erläutert werden. Ein Bayesianisches Gleichgewicht ist im Grunde genommen nichts anderes als eine spezielle Form von Nash-Gleichgewicht, also ganz allgemein ein Vektor von Strategien aller Spieler, die wechselseitig beste Antworten aufeinander bilden. Der Unterschied zu einem "gewöhnlichen" NashGleichgewicht läßt sich in etwa folgendermaßen erläutern:

Wenn ein Spieler $\mathrm{j}$ in dem oben beschriebenen Teilspiel zwischen den Agenten seine optimale Strategie festlegen will, muß er in der Lage sein, die Gleichgewichte dieses Spiels zu ermitteln. Unter der Bedingung vollständiger Information wäre dies relativ einfach, weil Spieler $j$ die Schadenskoeffizienten $\theta_{i}$ seiner Rivalen und damit deren Nutzenfunktionen $\mathrm{v}_{\mathrm{i}}\left(\mathrm{x}, \mathrm{z}, \theta_{\mathrm{i}}\right)$ beobachten könnte. Es wäre ihm also möglich, alle Strategiekombinationen $\mathrm{s}^{*}=\left(\mathrm{s}_{1}^{*}, \ldots, \mathrm{s}_{\mathrm{n}}^{*}\right)$ zu bestimmen, für die

$$
v_{i}\left[x\left(s_{i}^{*}, s_{-i}^{*}\right), z_{i}\left(s_{i}^{*}, s_{-i}^{*}\right), \theta_{i}\right] \geq v_{i}\left[x\left(s_{i}, s_{-i}^{*}\right), z_{i}\left(s_{i}, s_{-i}^{*}\right), \theta_{i}\right] \forall i=1 \ldots n, \forall s_{i} \in S_{i}
$$

gilt. Dabei ist $s_{-i}^{*}=\left(s_{1}^{*}, \ldots, s_{i-1}^{*}, s_{i+1}^{*}, \ldots, s_{n}^{*}\right)$. Ungleichung (3.7) ist die formale Beschreibung dafür, daß die Strategien $s_{i}^{*}$ wechselseitig beste Antworten bilden, also ein Gleichgewicht darstellen.

Bei unvollständiger Information kann Spieler $\mathrm{j}$ die Gleichgewichtstrategien nicht mehr wie in (3.7) ermitteln: Seine Opponenten haben je nach Ausprägung ihres Schadenskoeffizienten $\theta_{i}$ eine andere Nutzenfunktion, weshalb ihre Strategiewahl i.a. mit $\theta_{i}$ variieren wird. Da aber Spieler $\mathrm{j}$ die $\theta_{\mathrm{i}}$ a priori nicht kennt, sondern sie erst am Ende des Spiels erfährt, ist es ihm unmöglich vorauszusagen, wie sich seine Gegenspieler ex post verhalten werden. Er kann sich zu Beginn des Spiels allenfalls ausrechnen, welche Strategie ein Spieler bei einer bestimmten Realisation seines Schadenskoeffizienten ergreifen wird. Er muß also der Wahl seiner eigenen optimalen Strategie sog. "konditionale" oder "typabhängige" Strategien seiner Rivalen zugrunde legen. Eine typabhängige Strategie ist eine Funktion $\sigma_{i}\left(\theta_{i}\right)$, die jedem möglichen $\theta_{i} \in T_{i}$ eine Aktion $s_{i} \in S_{i}$ zuordnet. Das Verhalten von Spieler $i$ wird

${ }^{7}$ Für eine Einführung in diese Thematik vgl. z.B. Rasmusen (1989, S. 54 ff.) oder Fudenberg und Tirole (1991, S. 209ff.). 
also nicht durch eine einzelne Aktion, sondern durch je eine Aktion für jede Realisation von $\theta_{i}$ beschrieben. Mit Hilfe seines objektiven Wissens über die Dichtefunktionen $\mathrm{f}_{\mathrm{i}}\left(\theta_{\mathrm{i}}\right)$ kann sich dann jeder Spieler Erwartungen über das Verhalten seiner Gegenspieler bilden, seinen Erwartungsnutzen entsprechend bestimmen und selbst diejenige Strategie als beste Antwort wählen, die diesen Erwartungsnutzen maximiert.

Ein Bayesianisches Gleichgewicht ist dann ein Vektor $\left(\sigma_{1}\left(\theta_{1}\right), \ldots, \sigma_{n}\left(\theta_{n}\right)\right)$ typabhängiger Strategien, die wechselseitig beste Antworten bilden, d.h. gegeben den Strategievektor $\sigma_{-i}\left(\theta_{-i}\right)=\left(\sigma_{1}\left(\theta_{1}\right), \ldots, \sigma_{i-1}, \sigma_{i+1}, \ldots, \sigma_{n}\left(\theta_{n}\right)\right)$ der Opponenten muß $\sigma_{i}\left(\theta_{i}\right)$ den Erwartungsnutzen jedes Spielers i maximieren. Formal kann diese Bedingung wie folgt ausgedrückt werden:

$$
\begin{aligned}
& \int_{T_{-i}} v_{i}\left[x\left(\sigma_{i}\left(\theta_{i}\right), \sigma_{-i}\left(\theta_{-i}\right)\right), z_{i}\left(\sigma_{i}\left(\theta_{i}\right), \sigma_{-i}\left(\theta_{-i}\right)\right), \theta_{i}\right] f_{-i}\left(\theta_{-i}\right) d \theta_{-i} \geq \\
& \int_{T_{-i}}^{-i} v_{i}\left[x\left(s_{i}, \sigma_{-i}\left(\theta_{-i}\right)\right), z_{i}\left(s_{i}, \sigma_{-i}\left(\theta_{-i}\right)\right), \theta_{i}\right] f_{-i}\left(\theta_{-i}\right) d \theta_{-i} \quad \forall i=1 \ldots n, \\
& \forall \mathrm{s}_{\mathrm{i}}, \sigma_{\mathrm{i}}\left(\theta_{\mathrm{i}}\right) \in \mathrm{S}_{\mathrm{i}}\left(\mathrm{T}_{\mathrm{i}}\right)
\end{aligned}
$$

$\mathrm{S}_{\mathrm{i}}\left(\mathrm{T}_{\mathrm{i}}\right)$ bezeichnet dabei die Menge aller möglichen typabhängigen Strategien des Spielers i. Ein Bayesianisches Gleichgewicht wird also durch je einen Vektor $\left(s_{1}, \ldots, s_{n}\right)$ von Aktionen für jede denkbare Kombination $\theta \in T$ von Schadenskoeffizienten charakterisiert. Welcher Aktionsvektor tatsächlich gespielt wird, zeigt sich erst am Ende des Spiels, wenn klar ist, welche Spielertypen am Mechanismus teilgenommen haben. 8

Nach dieser kurzen allgemeinen Beschreibung des Gleichgewichtskonzepts für das Teilspiel zwischen den Geschädigten kann man sich der Frage zuwenden, wie der Schädiger einen Mechanismus, der seinen erwarteten Gewinn maximiert, gestalten soll. Ähnlich wie im zweiten Kapitel kann sein Entscheidungsproblem wieder durch die Anwendung des Enthüllungsprinzips vereinfacht werden. In bezug auf Mechanismen mit Bayesianischen Strategien besagt das Enthüllungsprinzip folgendes:

Jedes Ergebnis, das der Prinzipal mit einem beliebigen Mechanismus als Bayesianisches Gleichgewicht implementiern kann, kann auch mit einem direkten

${ }^{8}$ Zur Charakterisierung Bayesianischer Gleichgewichte vgl. beispielsweise auch Dasgupta, Hammond und Maskin (1979, S. 205f.), Myerson (1985, S. 240ff.), Repullo (1986, S.182) oder Fudenberg und Tirole (1991, S. 215). 
Enthüllungsmechanismus, in dem die wahrheitsgemäße Offenbarung der $\theta_{j}$ ein Bayesianisches Gleichgewicht darstellt, implementiert werden.

Auf einen Beweis dieses Satzes wird hier wegen der weitgehenden Analogie zum entsprechenden Beweis im zweiten Kapitel verzichtet. ${ }^{9}$ Es muß jedoch an dieser Stelle bereits darauf hingwiesen werden, da $B$ bei direkten Enthüllungsmechanismen typischerweise multiple Gleichgewichte auftreten, d.h. es existieren außer dem Gleichgewicht, in dem die Agenten ihre wahren Charakteristika offenbaren, u.U. noch andere, in denen sie lügen. Vor diesem Hintergrund muß nochmals ausdrücklich betont werden, daß das Enthüllungsprinzip direkte Enthüllungsmechanismen als Spielform nicht unbedingt nahelegt, sondern in erster Linie der Abgrenzung implementierbarer Ergebnisse dient. In der Literatur setzt man sich über das Problem multipler Gleichgewichte meist hinweg, indem man einfach annimmt, es werde das "wahrheitsgemäße Gleichgewicht" ("truthful equilibrium") gespielt.10 Dies ist aber nicht unproblematisch, weil die Gleichgewichte, in denen die Agenten lügen, höhere Nutzenniveaus für sie selbst und ein niedrigeres Nutzenniveau für den Prinzipal aufweisen können. Auf das Problem multipler Gleichgewichte sowie auf mögliche Ansatzpunkte zu seiner Beseitigung wird später noch etwas konkreter eingegangen.

\subsubsection{Die Verhandlungslösung mit mehreren Geschädigten}

In einem direkten Enthüllungsmechanismus ist die Menge $\mathrm{S}_{\mathrm{i}}$ der wählbaren $\mathrm{Si}$ gnale wieder identisch mit dem Intervall $T_{i}$ aller möglichen Ausprägungen des Grenzschadens $\theta_{\mathrm{i}}$. Die typabhängigen Strategien sind dann durch $\sigma_{\mathrm{j}}\left(\theta_{\mathrm{j}}\right)=\theta_{\mathrm{j}}{ }^{\prime}\left(\theta_{\mathrm{j}}\right)$ definiert, wobei $\theta_{j}{ }^{\prime}$.) ein bliebiger Schadenskoeffizient aus $T_{j}$ ist. Wenn die Angabe des wahren Grenzschadens durch alle Geschädigten ein Bayesianisches Gleichgewicht darstellen soll, dann muß es für einen Geschädigten i unter der Voraussetzung, da $\beta$ seine Opponenten $j \neq i$ ihr wahres $\theta_{j}$ angeben, optimal sein, seinerseits sein wahres $\theta_{i}$ anzugeben. Es muß also $\theta_{i}{ }^{\prime}\left(\theta_{i}\right)=\theta_{i} \forall i=1 \ldots n$ ein Gleichgewicht sein. Man erhält unter diesen Umständen in Anlehnung an (3.8) als Gleichgewichts- bzw. Anreizverträglichkeitsbedingung die folgende Ungleichung :

${ }^{9} \mathrm{Vgl.} \mathrm{z.B.} \mathrm{Dasgupta,} \mathrm{Hammond} \mathrm{und} \mathrm{Maskin} \mathrm{(1979,} \mathrm{S.} 206$ f.).

10Vgl. Rob (1989, S. 311). 


$$
\begin{aligned}
& \int_{T_{-i}} v_{i}\left[x\left(\theta_{i}, \theta_{-i}\right), z_{i}\left(\theta_{i}, \theta_{-i}\right), \theta_{i}\right] f_{-i}\left(\theta_{-i}\right) d \theta_{-i} \geq \\
& \int_{T_{-i}} v_{i}\left[x\left(\theta_{i}^{\prime}, \theta_{-i}\right), z_{i}\left(\theta_{i}^{\prime}, \theta_{-i}\right), \theta_{i}\right] f_{-i}\left(\theta_{-i}\right) d \theta_{-i}, \\
& \forall i=1 \ldots n, \theta_{i}, \theta_{i}^{\prime} \in T_{i} .
\end{aligned}
$$

Der Schädiger muß die Outputfunktion $\mathrm{x}$ und die Transferfunktionen $\mathrm{z}_{\mathrm{i}}$ so gestalten, $\mathrm{da}$ ß (3.9) erfüllt ist. Berücksichtigt man in dieser Bedingung die konkrete Spezifikation der Nutzenfunktionen $\mathrm{v}_{\mathrm{i}}\left(\mathrm{x}, \mathrm{z}_{\mathrm{i}}, \theta_{\mathrm{i}}\right)=\mathrm{z}_{\mathrm{i}}-\theta_{\mathrm{i}} \mathrm{x}$ und definiert

$$
u_{i}\left(\theta_{i}\right):=\int_{T_{-i}}\left\{z_{i}\left(\theta_{i}, \theta_{-i}\right)-\theta_{i} x(\theta)\right\} f_{-i}\left(\theta_{-i}\right) d \theta_{-i}
$$

dann lautet die Anreizverträglichkeitsbedingung

(AV) $\mathrm{u}_{\mathrm{i}}\left(\theta_{\mathrm{i}}\right) \geq \int_{\mathrm{T}_{-\mathrm{i}}}\left\{\mathrm{z}_{\mathrm{i}}\left(\theta_{\mathrm{i}}^{\prime}, \theta_{-\mathrm{i}}\right)-\theta_{\mathrm{i}} \mathrm{x}\left(\theta_{\mathrm{i}}^{\prime}, \theta_{-\mathrm{i}}\right)\right\} \mathrm{f}_{-\mathrm{i}}\left(\theta_{-\mathrm{i}}\right) \mathrm{d} \theta_{-\mathrm{i}} \forall \mathrm{i}=1 \ldots \mathrm{n}, \theta_{\mathrm{i}}, \theta_{\mathrm{i}}^{\prime} \in \mathrm{T}_{\mathrm{i}}$.

Damit die Teilnahme am Mechanismus von allen Geschädigten unabhängig von ihren Schadenskoeffizienten akzeptiert wird, muß wieder für jeden die Bedingung der individuellen Rationalität, also

(IR) $\mathrm{u}_{\mathrm{i}}\left(\theta_{\mathrm{i}}\right) \geq 0 \forall \mathrm{i}=1 \ldots \mathrm{n}, \theta_{\mathrm{i}} \in \mathrm{T}_{\mathrm{i}}$

gelten.

Die Gewinnfunktion des Schädigers, der nunmehr Transferzahlungen an $\mathrm{n}$ Geschädigte zu leisten hat, ist jetzt gegeben durch

(3.11) $E(\pi)=\int_{T}\left\{b(x(\theta))-\sum_{i=1}^{n} z_{i}(\theta)\right\} f(\theta) d \theta$.

Diese ist wieder unter den Bedingungen (AV) und (IR) zu maximieren.

Wie im zweiten Kapitel kann das Optimierungproblem des Schädigers vereinfacht werden, indem man Bedingungen angibt, die zu (AV) und (IR) äquivalent sind. Auf eine explizite Durchführung dieses Schrittes kann aber hier verzichtet werden, da die Vorgehensweise identisch ist mit der im zweiten Kapitel. Die Analogie wird unmittelbar deutlich, wenn man die Definitionen 
(3.12) $\mathrm{E}_{\mathrm{i}} \mathrm{z}_{\mathrm{i}}\left(\theta_{\mathrm{i}}\right):=\int_{\mathrm{T}_{-\mathrm{i}}} \mathrm{z}_{\mathrm{i}}\left(\theta_{\mathrm{i}}, \theta_{-\mathrm{i}}\right) \mathrm{f}_{-\mathrm{i}}\left(\theta_{-\mathrm{i}}\right) \mathrm{d} \theta_{-\mathrm{i}}$ und

(3.13) $E_{i} x\left(\theta_{i}\right):=\int_{\tau_{-i}} x\left(\theta_{i}, \theta_{-i}\right) f_{-i}\left(\theta_{-i}\right) d \theta_{-i}$

vornimmt. (AV) kann dann unter Verwendung dieser Definitionen auch wie folgt geschrieben werden:

$$
u_{i}\left(\theta_{i}\right)=E_{i} z_{i}\left(\theta_{i}\right)-\theta_{i} E_{i} x\left(\theta_{i}\right) \geq E_{i} z_{i}\left(\theta_{i}^{\prime}\right)-\theta_{i} E_{i} x\left(\theta_{i}^{\prime}\right) .
$$

Diese Ungleichung stimmt aber in ihrer Struktur mit der entsprechenden Ungleichung im Fall mit nur einem Geschädigten überein. ${ }^{11}$ Der Unterschied besteht lediglich darin, daß Output- und Transferfunktionen durch ihre bedingten Erwartungswerte $\mathrm{E}_{\mathrm{i}} \mathrm{x}\left(\theta_{\mathrm{i}}\right)$ bzw. $\mathrm{E}_{\mathrm{i}} \mathrm{z}_{\mathrm{i}}\left(\theta_{\mathrm{i}}\right)$ ersetzt wurden. Die Anforderungen, die im zweiten Kapitel an diese Funktionen gestellt wurden, müssen folglich jetzt von deren Erwartungswerten erfüllt werden. In Analogie zu den entsprechenden Ausführungen in Kapitel 2 gelten dann die Bedingungen

$$
u_{i}\left(\theta_{i}^{+}\right):=E_{i} z_{i}\left(\theta_{i}^{+}\right)-\theta_{i}^{+} E_{i} x\left(\theta_{i}^{+}\right) \geq 0
$$

(3.15) $\mathrm{E}_{\mathrm{i}} \mathrm{x}\left(\theta_{\mathrm{i}}\right)$ ist monoton fallend und

$$
u_{i}\left(\theta_{i}\right)=u_{i}\left(\theta_{i}^{+}\right)+\int_{\theta_{i}}^{\theta_{i}^{*}} E_{i} x\left(t_{i}\right) d t_{i}
$$

Im nächsten Schritt wird nun das Maximierungskalkül wieder durch Eliminierung der Transferfunktionen weiter vereinfacht. Nach einigen einfachen aber relativ mühsamen Umformungen, die in Anhang A nachzulesen sind, ergibt sich als modifizierte Zielfunktion

$$
E(\pi)=\int_{T}\left\{b(x(\theta))-x(\theta) \sum_{i=1}^{n}\left(\theta_{i}+\frac{F_{i}\left(\theta_{i}\right)}{f_{i}\left(\theta_{i}\right)}\right)\right\} f(\theta) d \theta-\sum_{i=1}^{n} u_{i}\left(\theta_{i}^{+}\right)
$$

$11 \mathrm{Vgl}$. Ungleichung (AV) auf S.41. 
Man sieht, da $ß$ die Transferfunktionen im Integranden des ersten Terms von (3.17) nicht mehr vorkommen, sondern nur noch implizit im Ausdruck $\sum_{i=1}^{n} u_{i}\left(\theta_{i}^{+}\right)$enthalten sind. Nach (3.16) muß nämlich $u_{i}\left(\theta_{i}^{+}\right)=u_{i}\left(\theta_{i}\right)-\int_{\theta_{i}}^{\theta_{i}^{+}} E_{i} x\left(t_{i}\right) d t_{i}$ gelten, woraus sich nach Einsetzen von (3.10) für $u_{i}\left(\theta_{i}\right)$ bzw. (3.13) für $E_{i} x\left(t_{i}\right)$

$$
u_{i}\left(\theta_{i}^{+}\right)=\int_{T_{-i}}\left\{z_{i}(\theta)-\theta_{i} x(\theta)-\int_{\theta_{i}}^{\theta_{i}^{+}} x\left(t_{i}, \theta_{-i}\right) d t_{i}\right\} f_{-i}\left(\theta_{-i}\right) d \theta_{-i}
$$

ergibt.

Wenn (3.17) maximiert werden soll, dann sollte $\sum_{i=1}^{n} u_{i}\left(\theta_{i}^{+}\right)$möglichst klein werden. Da alle Geschädigten unabhängig von ihrem Schadenstyp mindestens ihren Reservationsnutzen erhalten müssen, darf kein $u_{i}\left(\theta_{i}^{+}\right)$negativ sein. Die Summe wird also dann minimiert, wenn $u_{i}\left(\theta_{i}^{+}\right)=0$ für alle $i=1$...n gewählt wird. Aus Gleichung (3.18) ist ersichtlich, daß es hier, anders als im Fall mit nur einem Geschädigten, zahlreiche Möglichkeiten gibt, den Geschädigten i, falls er vom Typ $\theta_{i}^{+}$ist, auf sein Reservationsnutzenniveau zu drücken und gleichzeitig die Anreizverträglichkeit aller Kontrakte zu bewahren. Dies liegt daran, daß hier die Nutzenpositionen der Geschädigten unsicher sind, weil sie a priori nicht wissen, welche Schadensparameter ihre Gegenspieler angeben werden. Deshalb müssen (AV) und (IR) nur im Durchschnitt über alle möglichen Typen von Gegenspielern gelten, so daß es durchaus möglich wäre, daß der eine oder andere Geschädigte nach Bekanntwerden aller Schadensparameter bei bestimmten Konstellationen einen negativen Nutzen realisiert.

Wenn man Gleichung (3.18) betrachtet, liegt es allerdings recht nahe, die Transferfunktion gemäß

$$
z_{i}(\theta)=\theta_{i} x(\theta)+\int_{\theta_{i}^{*}}^{\theta_{i}^{+}} x\left(t_{i}, \theta_{-i}\right) d t_{i}
$$

zu wählen. Unter diesen Umständen erreicht jeder Geschädigte auch ex post für jede Konstellation von Schadensparametern ein nicht-negatives Nutzenniveau, weil er, wie in Kapitel 2, zusätzlich zum Schadensersatz $\theta_{i} x(\theta)$ wieder eine Informationsrente in Höhe von $\int_{\theta_{i}}^{\theta_{i}^{+}} x\left(t_{i}, \theta_{-i}\right) d t_{i}$ erhält. Man beachte aber, daß (3.19) nicht notwendige, sondern nur hinreichende Bedingung für ein Gewinnmaximum ist. 
Nun ist nur noch die gewinnmaximale Outputfunktion zu ermitteln. Dazu ist das Integral in (3.17) zu maximieren, wobei die Monotonierestriktion (3.15) beachtet werden müßte. Wenn man diese Restriktion zunächst wieder vernachlässigt, erhält man als notwendige Bedingung für ein Gewinnmaximum

$$
b^{\prime}(x(\theta))=\sum_{i=1}^{n}\left(\theta_{i}+\frac{F_{i}\left(\theta_{i}\right)}{f_{i}\left(\theta_{i}\right)}\right)
$$

Diese Optimalitätsbedingung ist derjenigen aus Kapitel 2 sehr ähnlich. Auf der rechten Seite der Gleichung muß jetzt natürlich die Summe der einzelwirtschaftlichen Grenzkosten einer Ausdehnung der Produktion über alle Geschädigten stehen. Die Interpretation der Summanden auf der rechten Seite von (3.20) ist identisch mit der aus Abschnitt 2.4: Die gesamten Grenzkosten einer Erhöhung von $\mathbf{x}(\theta)$ setzen sich zusammen aus der marginalen Erhöhung der Schadensersatzkomponenten und der Erhöhung der erwarteten Informationsrentenzahlungen für alle Geschädigten, deren Schadensparameter im Intervall $\left[\theta_{i} ; \theta_{i}\right]$ liegt. Die Summe der einzelwirtschaftlichen Grenzkosten ist höher als die Summe der Grenzschäden, die die gesamtwirtschaftlich relevanten Grenzkosten darstellen, so daß die gewählte Produktionsmenge im Vergleich zur effizienten Lösung wieder in fast allen Fällen zu niedrig ist. Ein effizientes Produktionsniveau wird nur in dem vernachlässigbaren Extremfall realisiert, in dem alle Geschädigten zufällig den niedrigsten Grenzschaden aufweisen, da nur in diesem Fall $\sum_{i=1}^{n} F_{i}\left(\theta_{i}\right) / f_{i}\left(\theta_{i}\right)=0$ wäre. Es bleibt noch anzumerken, daß die Monotonierestriktion (3.15) automatisch erfüllt ist, wenn die Quotienten $\mathrm{F}_{\mathrm{i}}\left(\theta_{\mathrm{i}}\right) / \mathrm{f}_{\mathrm{i}}\left(\theta_{\mathrm{i}}\right)$ monoton zunehmen, da unter diesen Umständen $\mathrm{x}(\theta)$ in allen $\theta_{\mathrm{i}}$ monoton abnimmt, so daß auch $\mathrm{E}_{\mathrm{i}} \mathrm{x}\left(\theta_{\mathrm{i}}\right)$ monoton abnimmt.

\subsection{Ein Beispiel: Das Modell von Rob}

Nachdem das bilaterale Verhandlungsmodell des zweiten Kapitels allgemein auf ein Szenario mit mehreren Geschädigten erweitert wurde, könnte man nun dazu übergehen, die Wohlfahrtseffekte einer Erhöhung der Anzahl der Geschädigten zu ermitteln. Um diese Wohlfahrtsanalyse technisch handhabbar zu halten, ist es aber nötig, das Modell noch weiter zu vereinfachen. Es wird sich zeigen, daß der formale Aufwand trotz dieser Vereinfachung, die im wesentlichen auf einer Spezifikation 
der Gewinnfunktion des Schädigers beruht, noch hoch genug ist. Durch diese Spezifikation erhält man erst den eigentlichen Modellrahmen, der der Arbeit von Rob (1989) zugrunde liegt.

Im nächsten Schritt wird die Funktionsweise des oben allgemein charakterisierten Mechanismus am Beispiel des Rob-Modells als Spezialfall etwas genauer untersucht. Für das Verständnis der Funktionsweise reicht die Betrachtung des Falls mit nur zwei Geschädigten vorerst aus. Die Beschränkung auf diesen Fall erlaubt es zudem, zur Verdeutlichung eine spezielle graphische Darstellungsmethode heranzuziehen und den relativ hohen Abstraktionsgrad von Robs Originaltext etwas zu senken. Diese graphische Darstellung ermöglicht es auch, die von Rob vorgeschlagenen $\mathrm{Maße}$ zur Beurteilung der Effizienzeigenschaften der privaten Verhandlungslösung bei $n$ Geschädigten auf einfache Weise abzuleiten.

\subsubsection{Der optimale Mechanismus}

Im folgenden wird angenommen, daß die Gewinnfunktion des Schädigers nur im Intervall $\left[0, x_{m}\right]$ konstante und positive Grenzgewinne aufweist. Es soll also

(3.21) $\quad b^{\prime}(x)=\left\{\begin{array}{lc}B>0 & \text { für } 0 \leq x \leq x_{m} \\ L<0 & \text { sonst }\end{array}\right.$

gelten.

Man könnte sich dabei beispielsweise vorstellen, daß bei einer Ausdehnung des Produktionsniveaus über $\mathrm{x}_{\mathrm{m}}$ hinaus Kapazitätserweiterungen vorgenommen werden müßten, die für den Schädiger unrentabel sind. Diese spezielle Form der Gewinnfunktion führt dazu, daß die Entscheidung über die gesamtwirtschaftlich optimale bzw. vom Schädiger gewählte Produktionsmenge binären Charakter erhält, da es nur noch darum geht, ob die Produktion des Gutes überhaupt aufgenommen werden soll oder nicht. Im ersten Fall wäre das Produktionsniveau $x_{m}$, im zweiten Fall $0 .{ }^{12}$

${ }^{12}$ Die Interpretation des Modells weicht von der in Robs Aufsatz ab. Rob nimmt an, das betrachtete Schädigerunternehmen könne bei Errichtung einer geplanten Produktionsanlage einen festen Ertrag erzielen. Gleichzeitig erleiden die Geschädigten im Falle einer Aufnahme der Produktion konstante Schäden. Die Entscheidungsvariablen des Schädigers sind dann die Kompensationszahlungen an die Geschädigten und eine Wahrscheinlichkeit $p$, mit der die Produktionsanlage errichtet 
Betrachten wir zunächst das gesamtwirtschaftliche Wohlfahrtsmaximierungskalkül: Eine Erhöhung des Produktionsniveaus ist lohnenswert, solange die gesamtwirtschaftlich relevanten Grenzkosten, also die Summe der individuellen Grenzschäden, $\sum_{i=1}^{n} \theta_{i}$, niedriger sind als der Grenzgewinn B des Schädigers. Da die Summe der individuellen Grenzschäden unabhängig von der Produktionsmenge ist, können nur zwei Fälle auftreten: Entweder ist $\sum_{i=1}^{n} \theta_{i} \leq B$, dann lohnt sich die Ausdehnung der Produktion bis $x_{m}$, oder es ist $\sum_{i=1}^{n} \theta_{i}>B$, dann sollte die Herstellung des Gutes gänzlich unterbleiben. Man erhält also in Abhängigkeit von $\theta=\left(\theta_{1}, \ldots, \theta_{n}\right)$

$$
x^{*}(\theta)=\left\{\begin{array}{cc}
x_{m} & \text { für } \sum_{i=1}^{n} \theta_{i} \leq B \\
0 & \text { sonst }
\end{array}\right.
$$

als effizientes Produktionsniveau.

Die Produktionsentscheidung des Schädigers würde sich nicht von (3.22) unterscheiden, wenn er die Schadenskoeffizienten beobachten könnte. Da ihm diese aber nicht bekannt sind, muß er den Geschädigten Informationsrenten zahlen, die einen Keil zwischen einzel- und gesamtwirtschaftliche Grenzkosten treiben, wie aus (3.20) hervorgeht. Dies führt dazu, daß die Produktionsentscheidung des Schädigers von der effizienten Lösung abweicht. Wenn man

$$
\left(\theta_{i}+\frac{F_{i}\left(\theta_{i}\right)}{f_{i}\left(\theta_{i}\right)}\right):=c_{i}\left(\theta_{i}\right)
$$

definiert, dann kann diese Produktionsentscheidung durch

$$
x_{v}(\theta)=\left\{\begin{array}{cc}
x_{m} & \text { für } \sum_{i=1}^{n} c_{i}\left(\theta_{i}\right) \leq B \\
0 & \text { sonst }
\end{array}\right.
$$

beschrieben werden.

wird. Auf die Übernahme dieser Interpretation wurde hier aus zwei Gründen verzichtet: Zum einen ist angesichts der Modellstruktur von vornherein klar, daß sich der Schädiger entweder für die Errichtung der Anlage oder den vollständigen Verzicht, also für $\mathrm{p}=1$ oder $\mathrm{p}=0$, entscheiden wird. Deshalb ist es m.E. etwas irreführend, a priori die Möglichkeit der Randomisierung, also $0<p<1$, zuzulassen. Zum anderen macht die hier gewählte Vorgehensweise deutlich, daß Robs Modell als Spezialfall eines allgemeineren Modells interpretiert werden kann. 
Ein Vergleich zwischen (3.22) und (3.24) zeigt, daß sich das Versagen der privaten Verhandlungslösung angesichts der speziellen Form der Gewinnfunktion nicht mehr wie bisher in einem ineffizient niedrigen Produktionsniveau äußert, sondern darin, daß bei bestimmten Konstellationen von Schadensparametern die Aufnahme der Produktion unterbleibt, obwohl sie aus gesamtwirtschaftlicher Perspektive sinnvoll wäre. Aus der Sicht des Unternehmens lohnt sich aber die Produktion wegen der zur Schadensenthüllung erforderlichen Anreizzahlungen nicht. Diese Ineffizienz tritt genau dann auf, wenn die Konstellation

$$
\sum_{i=1}^{n} \theta_{i} \leq B<\sum_{i=1}^{n} c_{i}\left(\theta_{i}\right)
$$

vorliegt.

Die Kompensationszahlungen, die der Schädiger leisten muß, erhält man aus (3.19). Falls $B<\sum_{i=1}^{n} c_{i}\left(\theta_{i}\right)$ ist, unterläßt der Schädiger die Herstellung des Gutes und hat auch keine Zahlungen zu leisten. Diese Feststellung ist nicht trivial: Es ist zwar klar, daß die Schadensersatzkomponente $\theta_{\mathrm{i}} \mathrm{x}(\theta)$ in diesem Fall hinfällig wird. Was jedoch die Informationsrenten betrifft, so wäre es prinzipiell denkbar, daß diese auch dann anfallen, wenn nicht produziert wird, da der Schädiger a priori nicht wissen kann, ob die Schäden hoch oder niedrig sind. Der Verlust, den der Schädiger unter diesen Umständen ex post erleiden würde, könnte ja durch höhere Gewinne bei anderen Realisierungen von Schadensparametern ausgeglichen werden, so daß der erwartete Gewinn immer noch positiv wäre. Diese Möglichkeit kommt jedoch hier nicht in Betracht. Da nämlich $x\left(\theta_{i}, \theta_{-i}\right)=0$ ist, muß $x\left(t_{i}, \theta_{-i}\right)$ für $t_{i}>\theta_{i}$ erst recht Null sein, so daß die Informationsrente $\int_{\theta_{i}}^{\theta_{i}^{*}} x\left(t_{i}, \theta_{-i}\right) d t_{i}$ ebenfalls gleich Null sein muß.

Gilt dagegen $\mathrm{B} \geq \sum_{\mathrm{i}=1}^{\mathrm{n}} \mathrm{c}_{\mathrm{i}}\left(\theta_{\mathrm{i}}\right)$, dann lohnt sich die Produktion für den Schädiger. Das Produktionsniveau ist in diesem Fall $x_{m}$. Die Schadensersatzkomponente muß jetzt nach (3.19) den Wert $\theta_{i} x_{m}$ annehmen. Bei der Bestimmung der Informationsrente sind zwei Fälle zu unterscheiden: Entweder ist $\theta_{i}^{+}$hinreichend klein, so daß $\sum_{j \neq i} c_{j}\left(\theta_{j}\right)+c_{i}\left(\theta_{i}^{+}\right) \leq B$ ist. Unter diesen Umständen würde sich die Produktion auch dann noch lohnen, wenn der Geschädigte $i$ bei gegebenem Vektor $\theta_{-i}$ den höchstmöglichen Grenzschaden $\theta_{i}^{+}$angeben würde. Es ist dann auch noch $x\left(\theta_{i}^{+}, \theta_{-i}\right)=x_{m}$, und die Informationsrente für den Geschädigten $i$ berechnet sich nach $\int_{\theta_{i}}^{\theta_{i}^{*}} x_{m} d t_{i}$. 
Andernfalls gibt es einen Schadenskoeffizienten $\theta_{i}^{\max }\left(\theta_{-i}\right)<\theta_{i}^{+}$, für den $\sum_{j \neq i} c_{j}\left(\theta_{j}\right)+c_{i}\left(\theta_{i}^{\max }\right)=B$ gilt. Dann ist aber $x\left(t_{i}, \theta_{-i}\right)=0$ für alle $t_{i}>\theta_{i}^{\max }\left(\theta_{-i}\right)$ und die Informationsrente bestimmt sich aus $\int_{\theta_{i}}^{\theta_{i}^{\text {max }}} x_{m} d t_{i}$.

Wenn man die jeweils zutreffende Informationsrente explizit berechnet und mit der Schadensersatzkomponente addiert, dann können die obigen Überlegungen zur Höhe der Kompensationszahlungen $\mathrm{z}_{\mathrm{i}}$ wie folgt zusammengefaßt werden:

$$
z_{i}(\theta)=\left\{\begin{array}{cc}
\theta_{i}^{+} x_{m} & \text { für } \sum_{i=1}^{n} c_{i}\left(\theta_{i}\right) \leq B \text { und } \theta_{i}^{+} \leq \theta_{i}^{\max }\left(\theta_{-i}\right) \\
\theta_{i}^{\max } x_{m} & \text { furr } \sum_{i=1}^{n} c_{i}\left(\theta_{i}\right) \leq B \text { Bund } \theta_{i}^{+}>\theta_{i}^{\max }\left(\theta_{-i}\right) \\
0 & \text { sonst. }
\end{array}\right.
$$

\subsubsection{Graphische Veranschaulichung des Mechanismus}

Die Funktionsweise des oben beschriebenen Mechanismus soll im folgenden graphisch verdeutlicht werden. Wir nehmen dazu der Einfachheit halber an, daß es nur $z w e i$ Geschädigte gibt, deren Schadenskoeffizienten $\theta_{\mathrm{i}}$ im Intervall $\left[0 ; \theta^{+}\right]$identisch und unabhängig gleichverteilt sind. Damit erhalten wir als Dichtefunktion

$$
f_{i}\left(\theta_{i}\right)=\left\{\begin{array}{cc}
1 / \theta^{+} & \text {für } 0 \leq \theta_{i} \leq \theta^{+} \\
0 & \text { sonst }
\end{array}\right.
$$

und als Verteilungsfunktion

$$
F_{i}\left(\theta_{i}\right)=\left\{\begin{array}{cc}
0 & \text { für } \theta_{i}<0 \\
\theta_{i} / \theta^{+} & \text {für } 0 \leq \theta_{i} \leq \theta^{+} \\
1 & \text { sonst. }
\end{array}\right.
$$

Darüber hinaus wird die Produktionsmenge $x_{m}$ zur Vereinfachung der Schreibweise auf Eins normiert. Unter diesen speziellen Bedingungen resultiert 
$(3.22)^{\prime} \quad x^{*}(\theta)=\left\{\begin{array}{lc}1 & \text { für } \theta_{1}+\theta_{2} \leq B \\ 0 & \text { sonst }\end{array}\right.$

als effiziente Lösung.

Die Produktionsentscheidung des Schädigers erhält man, wenn man berücksichtigt, daß aufgrund der Annahme einer Gleichverteilung der Schadenskoeffizienten $\sum_{i=1}^{2} c\left(\theta_{i}\right)=\sum_{i=1}^{2} \theta_{i}+F_{i}\left(\theta_{i}\right) / f_{i}\left(\theta_{i}\right)=2 \sum_{i=1}^{2} \theta_{i}$ gelten muß. Dann ergibt sich

$(3.24)^{\prime} \quad \mathrm{x}_{\mathrm{v}}(\theta)=\left\{\begin{array}{cc}1 & \text { für } \theta_{1}+\theta_{2} \leq \mathrm{B} / 2 \\ 0 & \text { sonst. }\end{array}\right.$

Betrachten wir dazu Abbildung 10.

Abbildung 10

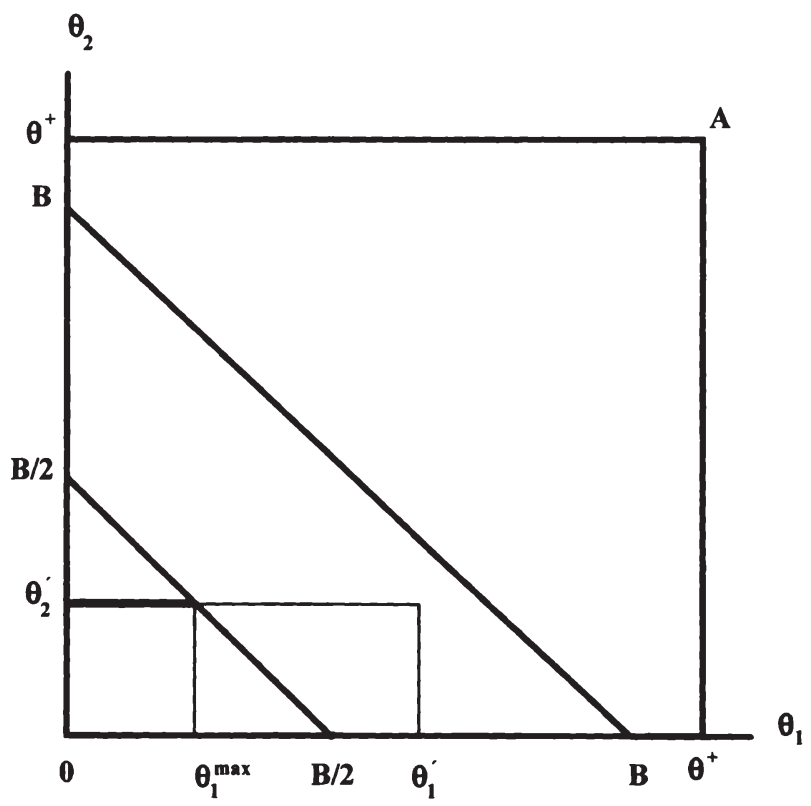


Das Quadrat $0 \theta^{+} \mathrm{A}^{+}$in dieser Abbildung bezeichnet alle Konstellationen von Schadensparametern, die überhaupt auftreten können. Gemäß (3.22)' wäre es gesamtwirtschaftlich betrachtet sinnvoll, die Produktion immer dann aufzunehmen, wenn eine Konstellation auf oder unterhalb der Geraden BB realisiert wird. Nach (3.24)' ist der Schädiger aber nur bereit zu produzieren, wenn eine Kombination auf oder unterhalb der Geraden $(B / 2)(B / 2)$ vorliegt. Wenn beispielsweise der zweite Geschädigte der Wahrheit entsprechend den Grenzschaden $\theta_{2}{ }^{\prime}$ angibt, dann produziert der Schädiger, solange der angegebene Grenzschaden des ersten Geschädigten den Wert $\theta_{1}^{\max }$ nicht überschreitet. Bei Bekundung eines höheren Schadenskoeffizienten, z.B. $\theta_{1}{ }^{\prime}$, unterbleibt die Herstellung des Gutes, obwohl sie aus gesamtwirtschaftlicher Sicht vorteilhaft wäre. Dies gilt im übrigen für all jene Kombinationen $\left(\theta_{1}, \theta_{2}\right)$ wahrheitsgemäß offenbarter Schadenskoeffizienten, die im Bereich zwischen den beiden Parallelen liegen.

Die Transferzahlung, die der erste Geschädigte erhält, berechnet sich nach (3.26), wobei zu beachten ist, $\mathrm{da} B \mathrm{~B} / 2<\theta^{+}$angenommen wurde, so daß für jedes gegebene $\theta_{2} \in\left[0 ; \theta^{+}\right]$immer eine Obergrenze $\theta_{1}^{\max }\left(\theta_{2}\right)$ existiert, bei deren Überschreitung der Schädiger nicht mehr bereit wäre, die Produktion aufzunehmen. ${ }^{13}$ Dieser Schwellenwert ist bestimmt durch $\theta_{1}^{\max }\left(\theta_{2}\right)=B / 2-\theta_{2}$, wie man unmittelbar der Graphik entnehmen kann. Da die gleiche Argumentation für den zweiten Geschädigten gilt, sind die Transferzahlungen durch

(3.26) $\quad \mathrm{z}_{\mathrm{i}}(\theta)=\left\{\begin{array}{cc}B / 2-\theta_{\mathrm{j}} & \text { für } \theta_{\mathrm{i}} \leq \mathrm{B} / 2-\theta_{\mathrm{j}} \\ 0 & \text { sonst }\end{array} \quad \mathrm{i}, \mathrm{j}=1,2 ; \mathrm{j} \neq \mathrm{i}\right.$

bestimmt. In Abbildung 10 kann die Transferzahlung, die der erste Geschädigte erhält, wenn der zweite Geschädigte den Grenzschaden $\theta_{2}$ ' angibt und er selbst einen Grenzschaden aus dem Intervall $\left[0 ; \theta_{1}^{\max }\right]$ nennt, durch die fettgedruckte Parallele zur Abszisse dargestellt werden. Falls $\theta_{1}>\theta_{1}^{\max }$ ist, erhält er nichts.

Unter Zuhilfenahme von Abbildung 11 wird nun illustriert, daß es in dem durch die Bedingungen (3.24)' und (3.26)' charakterisierten Mechanismus für den ersten Geschädigten optimal ist, seinen wahren Schadensparameter zu enthüllen, sofern der zweite Geschädigte ebenfalls die Wahrheit sagt.

${ }^{13}$ Diese Annahme ist unkritisch, erspart aber diverse Fallunterscheidungen bei der Berechnung der Informationsrenten. 


\section{Abbildung 11}

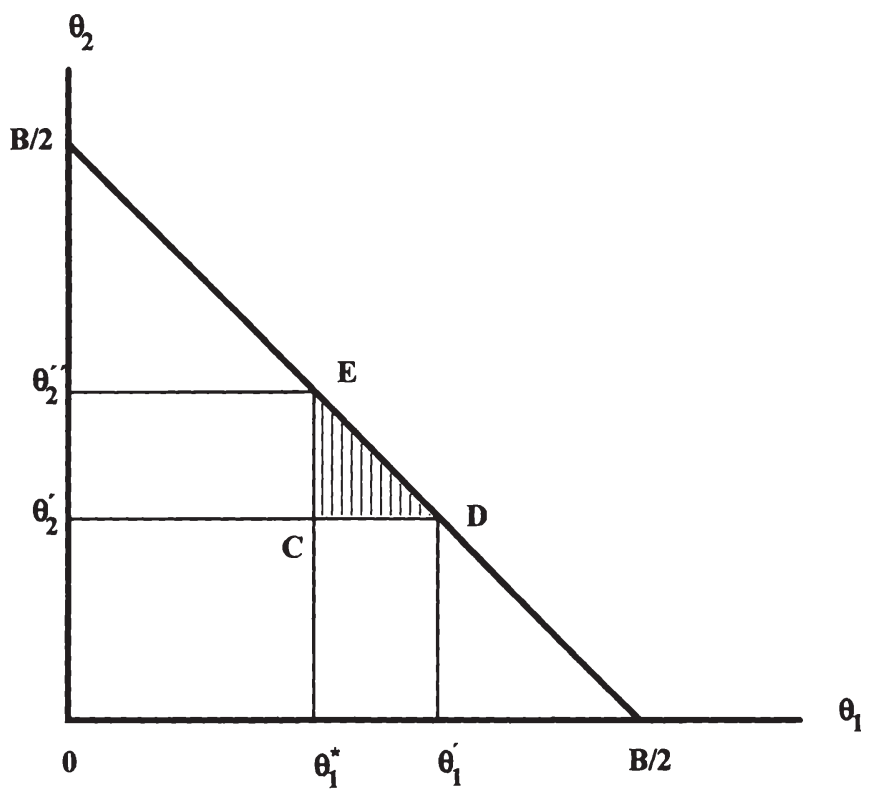

Wir nehmen dazu an, der wahre Grenzschaden des ersten Geschädigten sei $\theta_{1}{ }^{*}$ und zeigen zunächst, daß es sich nicht lohnt, einen höheren Grenzschaden $\theta_{1}{ }^{\prime}$ anzugeben.

Falls der erste Geschädigte wahrheitsgemäß $\theta_{1}{ }^{*}$ angibt, wird der Schädiger produzieren, solange der zweite Geschädigte ein $\theta_{2} \leq \theta_{2}{ }^{\prime \prime}$ angibt. Die Transferzahlungen, die in diesem Fall an den ersten fließen, belaufen sich nach (3.26)' unabhängig von $\theta_{1}$ auf $B / 2-\theta_{2}$. Falls der zweite Geschädigte ein $\theta_{2}>\theta_{2}{ }^{\prime \prime}$ angibt, unterbleiben Produktion und Zahlungen. Der erwartete Nutzen, den der erste Geschädigte bei korrekter Offenbarung seines Schadens realisiert, berechnet sich dann folgendermaßen:

$$
\begin{aligned}
& \mathrm{u}_{1}\left(\theta_{1}^{*}\right)=\int_{0}^{\theta^{+}}\left\{\mathrm{z}_{1}\left(\theta_{1}^{*}, \theta_{2}\right)-\theta_{1}^{*} \mathrm{x}\left(\theta_{1}^{*}, \theta_{2}\right)\right\} \mathrm{f}_{2}\left(\theta_{2}\right) \mathrm{d} \theta_{2}= \\
& =\int_{0}^{\theta_{2}^{*}}\left(B / 2-\theta_{2}\right) \mathrm{f}_{2}\left(\theta_{2}\right) \mathrm{d} \theta_{2}-\int_{0}^{\theta_{2}^{*}} \theta_{1}^{*} \mathrm{f}_{2}\left(\theta_{2}\right) \mathrm{d} \theta_{2} .
\end{aligned}
$$


Dieser Erwartungsnutzen kann in Abbildung 11 direkt identifiziert werden, wenn man $\theta^{+}=1$ setzt. Unter dieser Bedingung gilt nämlich $\mathrm{f}_{2}\left(\theta_{2}\right)=1$ für $\theta_{2} \in[0 ; 1]$, so daß schließlich nach Einsetzen von $\theta_{2}{ }^{\prime \prime}=B / 2-\theta_{1}{ }^{*}$ für die obere Integralgrenze

$$
u_{1}\left(\theta_{1}^{*}\right)=\int_{0}^{B / 2-\theta_{1}^{*}}\left(B / 2-\theta_{2}\right) d \theta_{2}-\int_{0}^{B / 2-\theta_{i}^{*}} \theta_{1}^{*} d \theta_{2}
$$

resultiert. Der erste Term dieser Gleichung steht für die erwarteten Transfers und entspricht der Fläche $0(\mathrm{~B} / 2) \mathrm{E} \theta_{2}{ }^{\prime \prime}$ in Abbildung 11. Der zweite Term gibt die erwartete Schadenshöhe an und wird durch die Fläche $0 \theta_{1}{ }^{*} \mathrm{E} \theta_{2}{ }^{\prime \prime}$ beschrieben. Der Nettonutzen bei korrekter Angabe von $\theta_{1}{ }^{*}$ ergibt sich also ganz einfach durch Integration der horizontalen Abstände zwischen der Geraden $(\mathrm{B} / 2)(\mathrm{B} / 2)$ und der Linie $\theta_{1}{ }^{*} \mathrm{E}$ und entspricht damit dem Inhalt der Fläche des Dreiecks $\theta_{1}{ }^{*}(\mathrm{~B} / 2) \mathrm{E}$.

Betrachten wir im Gegensatz dazu die Nutzenposition, die der Geschädigte erreicht, wenn er fälschlicherweise einen höheren Schaden angibt. Würde der Schädiger ihm nur einen Schadensersatz anbieten, hätte er nämlich einen Anreiz, seinen Schaden zu übertreiben: Bei wahrheitsgemäßer Enthüllung wäre sein Nutzen Null, während er im Falle einer Übertreibung bis $\theta_{1}{ }^{\prime}$ mit positiver Wahrscheinlichkeit eine positive Auszahlung erhielte. Er muß nur berücksichtigen, daß zwar der Vorteil $\theta_{1}{ }^{\prime}-\theta_{1}{ }^{*}$ umso höher ist, je stärker er übertreibt, daß aber gleichzeitig die Wahrscheinlichkeit für die Aufnahme der Produktion sinkt. Das optimale Ausmaß der Übertreibung wäre erreicht, wenn die marginale Erhöhung der Differenz zwischen angegebenem und tatsächlichem Schaden mit der marginalen Abnahme der Produktionswahrscheinlichkeit übereinstimmt. Nehmen wir an, diese Bedingung sei bei $\theta_{1}{ }^{\circ}$ in Abbildung 11 erfüllt.

Durch die Eigenschaften des Mechanismus ist gesichert, daß sich die Angabe von $\theta_{1}{ }^{\prime}$ nicht lohnt: Die Übertreibung führt dazu, daß die Wahrscheinlichkeit für die Aufnahme der Produktion im Vergleich zur korrekten Offenbarung von $\theta_{1}{ }^{*}$ abnimmt, weil der Schädiger schon für $\theta_{2}<\theta_{2}{ }^{\prime}<\theta_{2}{ }^{\prime \prime}$ nicht mehr bereit ist, zu produzieren. Dadurch reduziert sich einerseits der erwartete Schaden des ersten Geschädigten um die Fläche des Rechtecks $\theta_{2}{ }^{\prime \prime} \theta_{2}{ }^{\prime} \mathrm{CE}$. Andererseits vermindert sich aber auch die Höhe der zu erwartenden Transferzahlungen und zwar um den Inhalt der Trapezfläche $\theta_{2}{ }^{\prime \prime} \theta_{2}{ }^{\prime} D E$. Dies bedeutet, daß die Übertreibung dem Geschädigten einen Nutzenverlust in Höhe der schraffierten Dreiecksfläche CDE einbringen würde. Die ökonomische Erklärung für dieses Ergebnis ist offensichtlich: Bei Angabe von $\theta_{1}{ }^{\prime}$ ist die marginale Zunahme der erwarteten Transferzahlungen im Falle 
einer Senkung des signalisierten Schadenskoeffizienten gleich der Strecke $\theta_{2}{ }^{\prime} \mathrm{D}$ und damit höher als die marginale Erhöhung des erwarteten Schadens, die gerade der Strecke $\theta_{2}{ }^{\prime} \mathrm{C}$ entspricht. Deshalb lohnt sich eine Senkung des angegebenen Grenzschadens so lange, bis beide Größen übereinstimmen. Dies ist erst beim tatsächlichen Grenzschaden $\theta_{1}{ }^{*}$ der Fall.

Man könnte nun auf gleiche Weise zeigen, daß sich auch eine Untertreibung nicht lohnt. Wie man dabei vorgehen müßte, dürfte aber nach den obigen Ausführungen hinreichend klar sein, so daß sich eine explizite Durchführung erübrigt. $\mathrm{Da}$ das vorangegangene Argument umgekehrt auch für den zweiten Geschädigten gilt, ist es wechselseitig optimal, die wahren Schadensparameter anzugeben. Die korrekte Offenbarung stellt also ein Bayesianisches Gleichgewicht dar.

Die Nutzenpositionen, die die Geschädigten in diesem Gleichgewicht erreichen, können anhand von Abbildung 11 sehr leicht ermittelt werden. Man berechnet dazu die Dreiecksfläche $\theta_{1}{ }^{*}(\mathrm{~B} / 2) \mathrm{E}$, und setzt allgemein $\theta_{1}{ }^{*}=\theta_{\mathrm{i}}$. Man erhält dann

$$
u_{i}\left(\theta_{i}\right)=\left\{\begin{array}{cc}
1 / 2\left(B / 2-\theta_{i}\right)^{2} & \text { für } \theta_{i} \leq B / 2 \\
0 & \text { sonst. }
\end{array}\right.
$$

Den erwarteten Gewinn des Schädigers berechnet man am einfachsten aus Gleichung (3.17). Wenn man dabei berücksichtigt, daß $\mathrm{u}_{i}\left(\theta_{\mathrm{i}}^{+}\right)=0$ sein muß, und da $\beta$ die Produktion des Gutes nur aufgenommen wird, wenn die Realisation der Schadenskoeffizienten im Dreieck $0(\mathrm{~B} / 2)(\mathrm{B} / 2)$ in Abbildung 11 liegt, dann ergibt sich für unser spezielles Beispiel mit $x_{m}=1$ unter Berücksichtigung der Gleichverteilung $\operatorname{der} \theta_{\mathrm{i}}$ im Intervall $[0 ; 1]$ ein Gewinn in Höhe von

$$
\begin{aligned}
& E(\pi)=\int_{r}\left\{b(x(\theta))-x(\theta) \sum_{i=1}^{n}\left(\theta_{i}+\frac{F_{i}\left(\theta_{i}\right)}{f_{i}\left(\theta_{i}\right)}\right)\right\} f(\theta) d \theta= \\
& =\int_{0}^{B / 2} \int_{0}^{B / 2-\theta_{1}}\left[B-2\left(\theta_{1}+\theta_{2}\right)\right] d \theta_{2} d \theta_{1}=\frac{B^{3}}{24} .
\end{aligned}
$$

\subsubsection{Multiple Gleichgewichte}

Im vorangegangenen Teilabschnitt wurde gezeigt, daß im Rahmen des betrachteten Mechanismus die wahrheitsgemäße Offenbarung der Schadenskoeffizienten 
ein Bayesianisches Gleichgewicht darstellt. Es ist also für jeden Geschädigten optimal, seinen wahren Schaden zu signalisieren, wenn dies auch sein Gegenspieler tut. Die Betonung liegt dabei auf dem einschränkenden "wenn", denn möglicherweise ist dieses Bayesianische Gleichgewicht, das künftig auch "Enthüllungsgleichgewicht" genannt wird, nicht das einzige, das existiert. Wie schon mehrmals angedeutet, treten bei direkten Enthüllungsmechanismen im Teilspiel zwischen den Agenten typischerweise multiple Gleichgewichte auf. Falls dieses Phänomen auch in diesem Mechanismus zu beobachten ist, muß man sich kritisch mit der Frage auseinandersetzen, inwieweit das Enthüllungsgleichgewicht überhaupt als das Spielergebnis schlechthin aufgefaßt werden darf. Diese Problematik ist Gegenstand der Ausführungen dieses Teilabschnitts.

Im folgenden wird gezeigt, daß der Mechanismus in der Tat multiple Gleichgewichte aufweist. Dabei werden die speziellen Annahmen des vorangegangenen Teilabschnitts weitgehend aufrecht erhalten. Es wird aber anders als bisher unterstellt, daß $B / 2 \geq \theta^{+}$gelten soll. Diese Annahme erlaubt es, sehr leicht ein weiteres Gleichgewicht zu identifizieren, dessen Eigenschaften es besonders problematisch erscheinen lassen, das Enthüllungsgleichgewicht als tatsächliches Spielergebnis zu prognostizieren.

Bevor auf dieses Gleichgewicht eingegangen wird, muß zuerst das Enthüllungsgleichgewicht, das als Vergleichsmaßstab dienen soll, unter der Voraussetzung $\mathrm{B} / 2 \geq \theta^{+}$charakterisiert werden. Betrachten wir dazu Abbildung 12, die sich von Abbildung 11 nur im Hinblick auf die Relation zwischen $B / 2$ und $\theta^{+}$unterscheidet. Unter der Bedingung $B / 2 \geq \theta+$ liefert der Mechanismus im Enthüllungsgleichgewicht folgende Ergebnisse: Wenn der wahre Schadenskoeffizient des ersten Geschädigten durch $\theta_{1}{ }^{*}$ gegeben ist, dann nimmt der Schädiger die Produktion auf, sofern der Schadenskoeffizient des zweiten Geschädigten kleiner oder gleich $\theta_{2}{ }^{\prime \prime}$ ist. Der Transfer, der an den ersten Geschädigten fließt, ist gemäß (3.26) $z_{1}=\theta^{+}$, falls $\theta_{2} \leq \theta_{2}{ }^{\prime}$ bzw. $z_{1}=B / 2-\theta_{2}$, falls $\theta_{2}{ }^{\prime}<\theta_{2} \leq \theta_{2}{ }^{\prime \prime}$. Graphisch können diese Zahlungen in Abbildung 12 jeweils als horizontaler Abstand zwischen der Ordinate und der Senkrechten $\theta^{+} \mathrm{C}$ bzw. der Geraden $(B / 2)(B / 2)$ dargestellt werden. Den Nettonutzen $\mathrm{z}_{1}-\theta_{1}{ }^{*}$ erhält man für gegebenes $\theta_{2}$, indem man diesen Abstand jeweils um die Strecke $0 \theta_{1}{ }^{*}$ verkürzt. Ist $\theta_{2}>\theta_{2}{ }^{\prime \prime}$, dann produziert der Schädiger nicht, und es fließen keine Transfers. Der Nettonutzen ist dann gleich Null. Der erwartete Nettonutzen vor Bekanntwerden von $\theta_{2}$ ergibt sich analog zur Vorgehensweise in Abschnitt 3.3.2. Zu seiner graphischen Bestimmung 


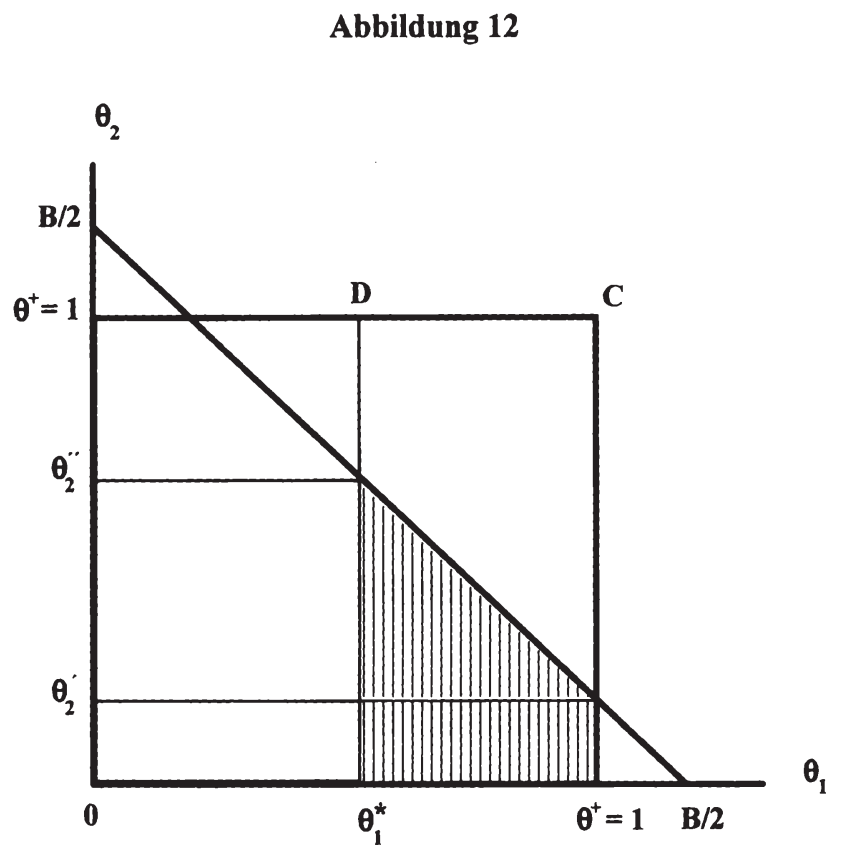

ist es wieder zweckmäßig, $\theta^{+}=1 \mathrm{zu}$ setzen. Unter dieser Bedingung entspricht der Erwartungsnutzen dem Inhalt der schraffierten Fläche in Abbildung 12.

Im nächsten Schritt wird nun gezeigt, daß neben der korrekten Offenbarung auch die Angabe eines Schadenskoeffizienten von Null durch jeden Geschädigten ein Gleichgewicht darstellt. Diese Aussage gilt, wie wir gleich sehen werden, wegen der Annahme $\mathrm{B} / 2 \geq \theta^{+}$unabhängig von den tatsächlichen Schadenstypen. Es muß also im folgenden bewiesen werden, daß die Signale $t_{i}=0$ für alle $\theta_{i} \in\left[0 ; \theta^{+}\right]$wechselseitig beste Antworten sind.

Angenommen, es sei die Strategie des zweiten Geschädigten, unter allen Umständen $t_{2}=0$ anzugeben. Nach den Regeln, die der Mechanismus vorschreibt, wird der Schädiger die Produktion aufnehmen und dem ersten Geschädigten einen Transfer in Höhe von $\theta^{+}$zahlen, gleich welches $t_{1} \in\left[0, \theta^{+}\right]$dieser signalisiert. Die Auszahlung, die er realisiert, ist also unabhängig von seinen eigenen Angaben. Beste Antworten auf $t_{2}=0$ sind damit alle $t_{1} \in\left[0, \theta^{+}\right]$, insbesondere auch $t_{1}=0$. Aus Gründen der Symmetrie ist natürlich auch $t_{2}=0$ beste Antwort auf $t_{1}=0$, weshalb das Strategiepaar $\left(t_{1}=0, t_{2}=0\right)$, unabhängig von den tatsächlichen Schadenskoeffizienten, 
ein Gleichgewicht bildet. ${ }^{14}$ Neben diesem Strategiepaar sind mit dem gleichen Argument alle Strategiepaare $\left(t_{1}, t_{2}\right)$ mit $t_{i} \leq B / 2$ ebenfalls Gleichgewichte.

Alle diese Gleichgewichte haben ein gemeinsames Merkmal: Die Transferzahlungen an die Geschädigten erreichen mit Sicherheit ihre maximale Höhe $\theta^{+}$. Dies ist aber ein Ergebnis, das bestimmt nicht im Interesse des Schädigers liegt, im Gegenteil: Es ist das denkbar ungünstigste Ergebnis, das er bei Aufnahme der Produktion erzielen kann. Die Auszahlungen der Geschädigten nehmen dagegen ihre Maximalwerte an. So erreicht beispielsweise der erste Geschädigte den deterministischen Nettonutzen $\theta^{+}-\theta_{1}{ }^{*}$, falls sein wahrer Schadensparameter $\theta_{1}{ }^{*}$ ist. Dieser Nutzen kann in Abbildung 12 durch die Fläche $\theta_{1}{ }^{*} \theta^{+} \mathrm{CD}$ dargestellt werden, wenn man wieder $\theta^{+}=1$ setzt. Ein Vergleich mit der erwarteten Nutzenposition im Enthüllungsgleichgewicht, die durch die schraffierte Fläche beschrieben wird, zeigt, daß sich der Geschädigte in dem Gleichgewicht, in dem er lügt, besserstellt.

Diese Beobachtung hat gravierende Folgen für die Relevanz des Enthüllungsgleichgewichts: $\mathrm{Da}$ ein Gleichgewicht existiert, in denen die Agenten höhere Auszahlungen realisieren als im Enthüllungsgleichgewicht, ist es unplausibel, davon auszugehen, daß gerade letzteres zustande kommen wird. Vielmehr haben die Agenten ein starkes Interesse daran, ihre Strategien so aufeinander abzustimmen, daß sich das Gleichgewicht durchsetzt, in dem sie höhere Nutzenniveaus erreichen. Falls es ihnen gelingt, dieses Koordinationsproblem zu lösen, verliert das Enthüllungsgleichgewicht jegliche Bedeutung. Dies wäre zwar in unserem Beispiel aus gesamtwirtschaftlicher Sicht durchaus wünschenswert, da es wegen $B / 2 \geq \theta^{+}$bzw. $B$ $\geq 2 \theta^{+}$in jedem Fall effizient wäre, die Produktion aufzunehmen. Dies würde auch geschehen, wenn beide Geschädigte beispielsweise den Schaden 0 angeben würden. $\mathrm{Da}$ aber ein solches Ergebnis nicht im Interesse des Schädigers liegt, stellt sich die Frage, welche Möglichkeiten er hat, die Implementierung des von ihm gewünschten Enthüllungsgleichgewichts doch zu sichern.

\footnotetext{
${ }^{14}$ Ausschlaggebend für dieses Resultat ist die Annahme $\mathrm{B} / 2 \geq \theta^{+}$. Dies wird unmittelbar klar, wenn man nochmals Abbildung 10 betrachtet, in der $\mathrm{B} / 2<\theta^{+}$unterstellt wurde. In diesem Fall ist die Angabe von 0 nicht unter allen Umständen ein Gleichgewicht: Ein Geschädigter mit $\theta_{i}>B / 2$ wird auf $t_{j}=0$ nie mit $t_{i}=0$ antworten, da der Schädiger sonst produzieren würde, er aber lediglich Zahlungen in Höhe von B/2 erhalten würde. Da sein Nutzen dann negativ wäre, wird er natürlich bestrebt sein, die Aufnahme der Produktion durch sein Signal zu verhindern. Beste Antwort auf $t_{j}=0$ ist also jedes $t_{i}>B / 2$, beispielsweise $t_{i}=\theta_{i}$. Robs Behauptung, $(0,0)$ sei immer ein Gleichgewicht (Vgl. Rob (1989, S. 319)), ist damit nicht richtig.
} 
Wenn man sich daran erinnert, daß die Gestaltung der Transferzahlungen nach (3.19) nur eine von vielen Alternativen war, eröffnet sich vielleicht ein Weg, das Problem multipler Gleichgewichte zu vermeiden. Der Schädiger könnte ja versuchen, die Transfers bei unveränderter Produktionsentscheidung so zu wählen, daß die Angabe der wahren Schadensparameter ein eindeutiges Gleichgewicht bildet. Dies könnte möglicherweise durch eine Veränderung des Zahlungsschemas dergestalt erreicht werden, daß die korrekte Offenbarung dominante Strategie für die Geschädigten ist. Daß dies unter bestimmten Umständen möglich ist, haben Mookherjee und Reichelstein (1992) gezeigt.

Eine andere Alternative, das Resultat des Enthüllungsgleichgewichts zu retten, wäre die Wahl einer völlig anderen Struktur des Teilspiels zwischen den Agenten. Die Notwendigkeit, einen direkten Enthüllungsmechanismus zu verwenden, besteht ja nicht unbedingt, denn das Enthüllungsprinzip besagt lediglich, daß dem Schädiger bei der Suche nach dem besten Kontrakt keine realisierbare Kombination von Transferzahlungen und Produktionsentscheidung entgeht, wenn er sich auf direkte Enthüllungsmechanismen konzentriert. Das heißt aber nicht, daß nicht andere, vielleicht komplexere Spielformen ebenso, wenn nicht gar besser dazu geeignet sind, das Ergebnis des Enthüllungsgleichgewichts $\mathrm{zu}$ implementieren. ${ }^{15}$ Der Schädiger müßte sich also eine Spielform überlegen, die dieses Ergebnis eindeutig implementiert. Welche Spielstrukturen dazu in Frage kommen, soll hier nicht weiter diskutiert werden. Eine Lösung wäre jedenfalls nicht leicht zu finden, da man keinerlei Anhaltspunkte hat, welche Spielform geeignet sein könnte. Rob selbst deutet an, daß eventuell mehrstufige Spiele in Betracht kommen könnten. ${ }^{16}$ Dieses Problem ist aber im Rahmen dieser Arbeit nur von sekundärem Interesse, da es hier in der Hauptsache um das Ergebnis, nicht um die konkrete Struktur des Spieles geht. Wenn wir davon ausgehen, daß es Möglichkeiten, welcher Art auch immer, gibt, das für den Schädiger optimale Ergebnis des Enthüllungsgleichgewichts auf anderem Wege zu implementieren, dann ändert sich im Grunde genommen nichts an den Aussagen über die Effizienzeigenschaften der Verhandlungslösung. Gibt es diese Möglichkeiten nicht, dann ist die Verhandlungslösung, wie sie hier dargestellt wurde, ohnehin hinfällig.

15 An dieser Stelle wird die Bedeutung des auf Seite 38 erwähnten Kreps-Zitats deutlich. ${ }^{16} \mathrm{Vgl}$. Rob (1989, S. 319). 


\subsubsection{Zwei Maße zur Beurteilung der Effizienz des Mechanismus}

Bei den folgenden Ausführungen wollen wir uns, wie in der Literatur üblich, nicht weiter um das Problem multipler Gleichgewichte kümmern, sondern davon ausgehen, daß es dem Schädiger möglich ist, eine Spielform zu finden, die die eindeutige Implementation der Resultate des Enthüllungsgleichgewichts gestattet. Damit können wir uns der Zielsetzung dieses und des nächsten Teilabschnitts zuwenden, die darin besteht, zu zeigen, daß sich die Effizienzeigenschaften der privaten Verhandlungslösung mit steigender Anzahl der Geschädigten verschlechtern. Um Aussagen über die Effizienz treffen zu können, benötigt man ein geeignetes $\mathrm{Ma} B$ zu deren Beurteilung. Rob bietet dazu in seiner Arbeit zwei Alternativen an, die sehr plausibel erscheinen.

Die erste Alternative besteht darin, die bei der Verhandlungslösung zu erwartenden Wohlfahrtsgewinne mit den prinzipiell möglichen ins Verhältnis zu setzen. Erstere setzen sich zusammen aus dem erwarteten Gewinn des Schädigers und dem Erwartungsnutzen der Geschädigten. ${ }^{17}$ Die prinzipiell erzielbaren Wohlfahrtsgewinne erhält man, wenn man das Allokationsproblem aus der hypothetischen Sicht eines wohlwollenden allwissenden Planers betrachtet. Ein solcher Planer würde für jede mögliche Realisation des Schadensvektors $\theta$ die First-Best-Lösung $x^{*}(\theta)$ wählen, die die gesamtwirtschaftliche Nettowohlfahrt $b(x)-x \sum_{i=1}^{n} \theta_{i}$ maximiert. Unter diesen Idealvoraussetzungen würde sich ex ante ein erwarteter Wohlfahrtsgewinn in Höhe von

$$
W^{*}=\int_{T}\left\{b\left(x^{*}(\theta)\right)-x^{*}(\theta) \sum_{i=1}^{n} \theta_{i}\right\} f(\theta) d \theta
$$

ergeben.

Im Rahmen unseres speziellen Beispiels aus Teilabschnitt 3.3.2, in dem ein konstanter Grenzgewinn unterstellt wurde, wurde die Planerlösung durch Gleichung (3.22)' charakterisiert. Gemäß dieser Gleichung ist die Aufnahme der Produktion des Gutes gesamtwirtschaftlich sinnvoll, wenn die Summe der Grenzschäden beider Geschädigten den Grenzgewinn des Schädigers nicht übersteigt. Dies ist dann der Fall, wenn das Tupel $\left(\theta_{1}, \theta_{2}\right)$ im Dreieck 0BB in Abbildung 13 liegt.

\footnotetext{
${ }^{17}$ Man beachte, daß die Berechnung der Wohlfahrtsgewinne hier ex ante, also vor Bekanntwerden der Schadensparameter, erfolgt.
} 
Abbildung 13

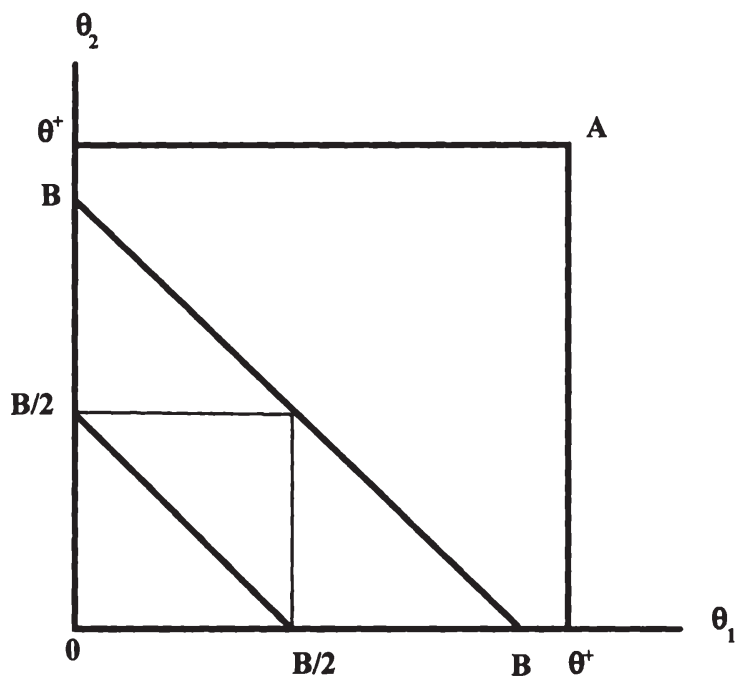

Wenn man berücksichtigt, daß das Produktionsniveau auf Eins normiert wurde, und wieder annimmt, daß die $\theta_{i}$ im Intervall $[0 ; 1]$ gleichverteilt sind, dann erhält man als potentiellen Wohlfahrtsgewinn in unserem Beispiel

$$
W^{*}=\int_{0}^{B} \int_{0}^{B-\theta_{1}}\left\{B-\left(\theta_{1}+\theta_{2}\right)\right\} d \theta_{2} d \theta_{1}=\frac{B^{3}}{6}
$$

Der erwartete Wohlfahrtsgewinn, der im Rahmen der privaten Verhandlungen erzielt wird, ist allgemein definiert durch

$$
\mathrm{W}=\mathrm{E}(\pi)+\sum_{\mathrm{i}=1}^{\mathrm{n}} \mathrm{Eu}_{\mathrm{i}}\left(\theta_{\mathrm{i}}\right)
$$

Für das Beispiel wurden bereits $E(\pi)$ in (3.30) und $u_{i}\left(\theta_{i}\right)$ in (3.29) bestimmt. Um W zu erhalten, müssen noch die Durchschnitte der Erwartungsnutzen $u_{i}\left(\theta_{i}\right)$ über alle $\theta_{i}$ gebildet werden. Diese resultieren unter Berücksichtigung von (3.29) aus 


$$
\begin{gathered}
\operatorname{Eu}_{i}\left(\theta_{i}\right)=\int_{0}^{\theta^{+}} u_{i}\left(\theta_{i}\right) f_{i}\left(\theta_{i}\right) d \theta_{i}=\int_{0}^{B / 2} u_{i}\left(\theta_{i}\right) d \theta_{i}= \\
=\frac{1}{2} \int_{0}^{B / 2}\left(\frac{B}{2}-\theta_{i}\right)^{2} d \theta_{i}=\frac{B^{3}}{48} .
\end{gathered}
$$

Damit resultiert

$$
\mathrm{W}=\frac{\mathrm{B}^{3}}{24}+\frac{2 \mathrm{~B}^{3}}{48}=\frac{\mathrm{B}^{3}}{12}
$$

als erwarteter Wohlfahrtsgewinn bei der Verhandlungslösung.

Das angestrebte Wohlfahrtsmaß erhält man schließlich, wenn man (3.32) durch (3.31) dividiert:

$$
\omega_{1}:=\frac{\mathrm{W}}{\mathrm{W}^{*}}=\frac{1}{2}
$$

Es zeigt sich also, daß unter den speziellen Bedingungen unseres Beispiels bei privaten Verhandlungen im Durchschnitt nur die Hälfte des potentiellen Wohlfahrtsgewinns realisiert wird.

Das Wohlfahrtsma $\beta \omega_{1}$ ist nicht nur im engen Rahmen dieses Beispiels anwendbar, wenngleich die Annahme eines konstanten Grenzgewinns erhebliche Erleichterungen bei der Berechnung der einzelnen Komponenten mit sich bringt. Der Verzicht auf diese Annahme hätte zur Folge gehabt, daß die Produktionsentscheidung des Schädigers ihren binären Charakter verloren hätte. Die erforderlichen wahrscheinlichkeitstheoretischen Berechnungen wären in diesem Fall erheblich aufwendiger gewesen. Vor diesem Hintergrund wird der Sinn der Annahme eines konstanten Grenzgewinns deutlich. Prinzipiell könnte $\omega_{1}$ aber auch unter komplizierteren Bedingungen verwendet werden.

Der Anwendungsbereich des zweiten Wohlfahrtsmaßes, das nun vorgestellt werden soll, ist dagegen auf den Fall konstanter Grenzgewinne beschränkt. Wie in Abschnitt 3.3.1 erläutert wurde, äußert sich die Ineffizienz der Verhandlungslösung unter diesen Umständen darin, daß der Schädiger bei bestimmten Konstellationen von Schadenskoeffizienten nicht bereit ist, das fragliche Gut herzustellen, obwohl dies aus gesamtwirtschaftlicher Perspektive sinnvoll wäre. Das Gut sollte produziert werden, wenn die Summe der Schadenskoeffizienten aller Geschädigten kleiner 
oder gleich dem Grenzgewinn des Schädigers ist. Dieser ist aber nur bereit zu produzieren, wenn die Summe seiner privaten Grenzkosten, die die gesamtwirtschaftlichen Grenzkosten um die marginalen Enthüllungszahlungen übersteigen, nicht höher ist, als der Grenzgewinn. Damit bietet sich als Wohlfahrtsmaß die Wahrscheinlichkeit an, mit der der Schädiger die Produktion aufnimmt, sofern dies gesamtwirtschaftlich lohnenswert ist. Je näher diese Wahrscheinlichkeit bei Eins liegt, desto besser ist die Verhandlungslösung zur Internalisierung des externen Effekts geeignet. Diese bedingte Wahrscheinlichkeit ist allgemein definiert durch

$$
\omega_{2}=\mathrm{P}\left(\sum_{\mathrm{i}=1}^{2} \mathrm{c}_{\mathrm{i}}\left(\theta_{\mathrm{i}}\right) \leq \mathrm{B} / \sum_{\mathrm{i}=1}^{2} \theta_{\mathrm{i}} \leq \mathrm{B}\right) .
$$

Wenn man wieder wie bisher annimmt, da $\beta$ die $\theta_{i}$ im Intervall $[0 ; 1]$ unabhängig und identisch gleichverteilt sind, dann sind die privaten Grenzkosten des Schädigers $c_{i}\left(\theta_{i}\right)=2 \theta_{i}$, und $\omega_{2}$ wird $\mathrm{zu}$

$$
\omega_{2}=\mathrm{P}\left(2 \sum_{\mathrm{i}=1}^{2} \theta_{\mathrm{i}} \leq \mathrm{B} / \sum_{\mathrm{i}=1}^{2} \theta_{\mathrm{i}} \leq \mathrm{B}\right)=\frac{\mathrm{P}\left(\sum_{\mathrm{i}=1}^{2} \theta_{\mathrm{i}} \leq \mathrm{B} / 2\right)}{\mathrm{P}\left(\sum_{\mathrm{i}=1}^{2} \theta_{i} \leq \mathrm{B}\right)}
$$

Zur Berechnung von $\omega_{2}$ ist es hilfreich, Abbildung 13 heranzuziehen. Da wegen der Gleichverteilungsannahme jede Kombination $\left(\theta_{1}, \theta_{2}\right)$ im Quadrat $0 \theta^{+} A \theta^{+}$die gleiche Wahrscheinlichkeitsdichte besitzt, ist unmittelbar klar, da $B \omega_{2}$ durch das Verhältnis der Dreiecksfläche $0(\mathrm{~B} / 2)(\mathrm{B} / 2)$ zur Dreiecksfläche $0 \mathrm{BB}$ ausgedrückt werden kann. Es ist offensichtlich, daß dann $\omega_{2}=1 / 4$ sein muß. D.h. im Rahmen der privaten Verhandlungslösung produziert der Schädiger nur in $25 \%$ der Fälle, in denen es gesamtwirtschaftlich geboten erscheint.

\subsubsection{Wohlfahrtseffekte bei Erhöhung der Anzahl der Geschädigten}

Nach den Ausführungen des vorangegangenen Teilabschnitts sind wir nunmehr in der Lage, eines der zentralen Ergebnisse dieses Kapitels herzuleiten. Es soll gezeigt werden, daß die Effizienzgewinnne, die im Rahmen privater Verhandlungen über Externalitäten erzielt werden können, verschwinden, wenn die Anzahl der Geschädigten gegen Unendlich geht. 
Damit dieses Ergebnis nicht trivial wird, ist nochmals eine geringfügige Modifikation des Modellrahmens erforderlich. Es wäre unsinnig, die Anzahl der Geschädigten einfach gegen Unendlich streben zu lassen, während der Gewinn des Schädigers konstant gehalten wird. In diesem Fall ist völlig klar, daß die Produktion des Gutes ab einer bestimmten Gesamtsumme aller Schäden nicht einmal mehr aus gesellschaftlicher Perspektive, geschweige denn aus der Sicht des Schädigers rentabel wäre. Es ist also sinnvoll, den Gewinn des Schädigers ebenfalls mit der Anzahl der Geschädigten wachsen zu lassen. Die einfachste Möglichkeit der Modellierung einer von der Zahl der Geschädigten abhängigen Gewinnfunktion ist zu unterstellen, daß der Gewinn pro Geschädigten konstant sei. Die Grenzgewinnfunktion des Schädigers ist in diesem Fall analog zu (3.21) durch

$$
b^{\prime}(x)=\left\{\begin{array}{cc}
\beta n>0 & \text { für } 0 \leq x \leq 1 \\
\lambda<0 & \text { sonst }
\end{array}\right.
$$

gegeben. Man kann sich bei dieser Modellierung der Gewinne des Schädigers beispielsweise vorstellen, da $\mathrm{n}$ identische Nachfrager maximal eine Einheit seines Produkts nachfragen, wobei $\beta$ den Zusatzgewinn pro Geschädigten bei einer marginalen Erhöhung der Produktion im Intervall $[0 ; 1]$ bezeichnet. Hinter dieser Modellierung steht die Vorstellung, daß mit der Anzahl der Geschädigten die gesamte Ökonomie wächst. Rob führt seine Analysen deshalb unter der Überschrift "Mechanism Performance In Large Economies" durch.18

Man kann nun dazu übergehen, die im vorangegangenen Teilabschnitt für den Fall zweier Geschädigter eingeführten Wohlfahrtsmaße $\omega_{1}$ und $\omega_{2}$ für $n$ Geschädigte $\mathrm{zu}$ ermitteln und $\mathrm{n}$ dann gegen Unendlich streben lassen. Dies soll hier explizit nur für das einfacher zu handhabende $\mathrm{Ma} \beta \omega_{2}$ durchgeführt werden. Was $\omega_{1}$ betrifft, so müßte man im Prinzip den erwarteten Gewinn des Schädigers, die durchschnittlichen Nutzenniveaus der Geschädigten und die erwartete Wohlfahrt bei Realisierung der First-Best-Allokation in Abhängigkeit von $\mathrm{n}$ bestimmen, daraus

$$
\omega_{1}(n)=\frac{E(\pi(n))+\sum_{i=1}^{n} E u_{i}\left(\theta_{i}, n\right)}{W^{*}(n)}
$$

${ }^{18}$ Vgl. Rob (1989, S. 319). 
bilden und $\lim _{n \rightarrow \infty} \omega_{1}(n)$ berechnen. Der Rechenaufwand ist dabei aber erheblich, so daß es sich m.E. nicht lohnt, die Schritte im einzelnen durchzuführen, zumal das Resultat dasselbe ist wie für $\omega_{2}$. Es sei deshalb nur auf die entsprechenden Ausführungen bei Rob (1989), insbesondere auf den Beweis seines Theorems 3 im Anhang hingewiesen. Dieses Theorem besagt, daß unter nicht allzu strengen Annahmen an die Wahrscheinlichkeitsverteilungen $\operatorname{der} \theta_{i} \lim _{n \rightarrow \infty} \omega_{1}(n)=0$ ist. Daneben ist noch erwähnenswert, da $B \omega_{1}(n)$ offensichtlich bereits für recht kleine $n$ rapide abnimmt. So zeigen Robs Simulationen eines speziellen numerischen Beispiels, daß je nach Höhe des Pro-Kopf-Gewinns $\beta$ der Anteil der realisierten Wohlfahrtsgewinne an den potentiellen bereits ab $n=10$ unter $10 \%$ liegen kann. D.h., $n$ muß gar nicht unendlich groß werden, damit private Verhandlungen praktisch keine Effizienzgewinne mehr bringen.

Wir wollen nun das Verhalten des zweiten Wohlfahrtsmaßes $\omega_{2}$ untersuchen, wenn $\mathrm{n}$ gegen Unendlich strebt. Wir müssen dazu nur berücksichtigen, daß der Grenzgewinn des Schädigers nunmehr durch $\beta \mathrm{n}$ gegeben ist und die Bedingungen für die Rentabilität der Aufnahme der Produktion aus einzel- bzw. gesamtwirtschaftlicher Sicht entsprechend modifizieren: Die Produktion des Gutes wäre gesamtwirtschaftlich rentabel, wenn $\sum_{i=1}^{n} \theta_{i} \leq \beta n$ ist, während der Schädiger nur dann produziert, wenn $\sum_{\mathrm{i}=1}^{\mathrm{n}} \mathrm{c}_{\mathrm{i}}\left(\theta_{\mathrm{i}}\right) \leq \beta \mathrm{n}$ ist. Um den formalen Aufwand in Grenzen zu halten, sei wieder angenommen, daß die $\theta_{i}$ im Intervall $[0 ; 1]$ unabhängig und identisch gleichverteilt sind. ${ }^{19}$ Unter dieser Voraussetzung erhalten wir

$$
\omega_{2}(n)=P\left(2 \sum_{i=1}^{n} \theta_{i} \leq \beta n / \sum_{i=1}^{n} \theta_{i} \leq \beta n\right)=\frac{P\left(2 \sum_{i=1}^{n} \theta_{i} \leq \beta n\right)}{P\left(\sum_{i=1}^{n} \theta_{i} \leq \beta n\right)} .
$$

Die Bestimmung von $\lim _{n \rightarrow \infty} \omega_{2}(n)$ ist nicht allzu schwierig. Die nötigen Berechnungen sind aber etwas langwierig und gewähren wenig Aufschluß über ökonomische Inhalte. Sie wurden deshalb in Anhang B explizit durchgeführt. Wie man dort nachlesen kann, geht in der Tat auch $\omega_{2}(n)$ gegen Null, wenn $\mathbf{n}$ gegen Unendlich geht. Es zeigt sich also, daß in diesem speziellen Beispiel die Wahrscheinlichkeit dafür, daß der Schädiger die Produktion aufnimmt, wenn er dies aus gesamtwirt-

${ }^{19}$ Die Gleichverteilung erfüllt die von Rob bei seiner allgemeineren Analyse getroffenen Verteilungsannahmen. (Vgl. Rob (1989, S. 321 f.)). 
schaftlicher Perspektive tun sollte, bei einer sehr hohen Anzahl von Geschädigten Null wird.

Die Annahmen, die diesem Beispiel zugrundeliegen, sind sehr restriktiv. Es stellt sich deshalb die Frage, ob das Ergebnis auch auf allgemeinere Fälle übertragbar ist. Die spezielle Verteilungsannahme, die hier getroffen wurde, ist nicht besonders kritisch, da sich, wie Rob gezeigt hat, unter weitaus schwächeren Annahmen das gleiche Resultat einstellt. Welche Bedeutung die Form der Abhängigkeit der Gewinnfunktion von der Anzahl der Geschädigten hat, ist dagegen unklar. Rob selbst gesteht zu, daß die Allgemeingültigkeit über diesen Spezialfall hinaus nicht gesichert ist. ${ }^{20}$ Es gibt aber abgesehen von den Modellrechnungen auch eine intuitive Erklärung, die Robs Ergebnis plausibel macht und Anlaß zur Vermutung gibt, daß es seine Gültigkeit über den Modellrahmen hinaus zumindest in der Tendenz behält:

Da der Schädiger den Geschädigten das Recht zur Ausübung des externen Effekts abkaufen muß, hätten diese bei einer reinen Schadensersatzregelung ursprünglich einen Anreiz, ihren Schaden zu übertreiben, um sich eine positive Auszahlung zu sichern. Ist ihre Anzahl gering, so muß ein einzelner Geschädigter damit rechnen, daß die Wahrscheinlichkeit für die Aufnahme der Produktion umso niedriger sein wird, je höher sein angegebener Schaden ist. Der Vorteil, der durch eine Übertreibung erlangt werden kann, ist also dadurch begrenzt, daß der Erwartungswert der Schadensersatzzahlungen wieder abnimmt, wenn zu stark übertrieben wird. Dementsprechend sind auch die Informationsrenten, die der Schädiger im Enthüllungsmechanismus zu zahlen hat, um den Geschädigten den Anreiz zur Übertreibung zu nehmen, begrenzt.

Ist dagegen die Anzahl der Geschädigten sehr hoch, dann ist der Einfluß eines einzelnen auf die Produktionsentscheidung des Schädigers vernachlässigbar gering. Gegeben die Schadensmeldungen seiner Opponenten könnte ein Geschädigter seinen eigenen Schaden bis an die Grenze der Glaubwürdigkeit übertreiben, ohne da $B$ dadurch die Wahrscheinlichkeit für die Aufnahme der Produktion spürbar sinken würde. Sein Anreiz zu übertreiben ist also in dieser Situation viel stärker ausgeprägt als im Fall mit wenigen Geschädigten. Die Informationsrente pro Geschädigten, die diesen Anreiz beseitigen soll, muß also entsprechend höher sein, so daß die Produktion für den Schädiger immer unrentabler wird.

${ }^{20} \mathrm{Vgl.} \mathrm{Rob}(1989$, S. 320). 
Ob diese Tendenz dazu führt, daß überhaupt nicht produziert wird, hängt in entscheidendem $\mathrm{Ma} B$ davon $\mathrm{ab}$, wie sich der Gewinn pro Geschädigten entwickelt, wenn deren Zahl zunimmt. Rob hat angenommen, daß der Pro-Kopf-Gewinn konstant bleibt. Es ist also zu erwarten, daß sich sein Ergebnis erst recht bei sinkendem Pro-Kopf-Gewinn einstellt, während es sich im Falle eines steigenden Pro-KopfGewinns abschwächen wird. Dies ist aber nicht der entscheidende Punkt. Die wichtigste Erkenntnis ist die, daß das Informationsproblem durch die Vielzahl der $\mathrm{Ge}$ schädigten verschärft wird. Diese Erkenntnis ist aber weitgehend unabhängig von den Spezialannahmen des Modells und dürfte auch für formal komplexere Szenarien, in denen die Produktionsentscheidung des Schädigers nicht binärer Natur ist, zutreffen.

Vor dem Hintergrund dieser Überlegungen läßt sich auch der diametrale Gegensatz zu den eingangs dieses Kapitels zitierten Beiträgen von Wilson (1985) und Gresik und Satterthwaite (1989) zur Auktionstheorie erklären: Werden, wie in diesen Auktionsmodellen, private Güter gehandelt, dann erweist sich die Vielzahl der Auktionsteilnehmer als effizienzsteigernd. Je mehr Nachfrager und Anbieter an der Versteigerung teilnehmen, desto geringer ist der Einfluß des einzelnen auf den Verkaufspreis. Dadurch schwächt sich der Anreiz, eine falsche Wertschätzung für die gehandelten Objekte anzugeben, ab, da eine Falschangabe nur die Wahrscheinlichkeit, ein Objekt kaufen bzw. verkaufen zu können, senkt.

In Robs Modell wird dagegen das Recht zur Ausübung eines externen Effekts gehandelt, der aus der Sicht der Geschädigten ein öffentliches Gut darstellt. Diesem Umstand ist es zuzuschreiben, daß die Vielzahl der Geschädigten effizienzmindernd wirkt, weil eine übertriebene Schadensmeldung durch ein einzelnes Individuum keine nennenswerte Auswirkung auf die Produktionsentscheidung des Schädigers hat. Die Anreize, Falschangaben zu machen, nehmen dann zu, und die Informationsrenten im Enthüllungsmechanismus, die Falschangaben verhindern sollen, werden in die Höhe getrieben.

Es zeigt sich also, daß im Verhandlungsmodell mit mehreren Geschädigten, in dem das Verursacherprinzip gilt und der Schädiger das Vertragsangebot unterbreiten kann, doch wieder eine spezielle Form von Trittbrettfahrerverhalten auftritt. Dieses manifestiert sich in dem Bestreben jedes einzelnen Geschädigten, sich in Abwesenheit geeigneter Gegenmaßnahmen durch übertriebene Schadensmeldungen nicht nur auf Kosten des Schädigers, sondern insbesondere auch auf Kosten seiner Leidensgenossen Vorteile zu verschaffen. 
Ein ähnliches Argument gilt in abgewandelter Form auch bei Zugrundelegung des Laisser-Faire-Prinzips. In diesem Fall müßten die Geschädigten Zahlungen leisten und hätten deshalb ursprünglich einen Anreiz, ihren Schaden zu untertreiben. In Gegenwart vieler Geschädigter wäre dieser Anreiz sehr hoch, da der einzelne davon ausgehen könnte, daß bei gegebenen Schadensmeldungen seiner Opponenten seine eigene Untertreibung die Wahrscheinlichkeit für die Aufnahme der Produktion praktisch nicht erhöhen würde. Er selbst könnte dagegen seine Zahlungen auf ein Mindestma $\beta$ senken. Die Vorteile einer Falschangabe sind also auch hier wieder größer als im Fall mit wenigen Geschädigten, so daß der Schädiger den Geschädigten wieder hohe Informationsrenten zugestehen müßte. Dies würde sich darin äußern, daß die Zahlungen, die an ihn fließen, nicht hoch genug sind, um eine nennenswerte Senkung der Produktionswahrscheinlichkeit herbeizuführen. Die Ineffizienz der Verhandlungslösung, die sich darauf gründet, daß der Schädiger bei manchen Konstellationen von Schadensparametern die Produktion aufnimmt, obwohl dies aus gesamtwirtschaftlicher Sicht nicht sinnvoll ist, wird also durch die Vielzahl der Geschädigten wieder verschärft.

Als Fazit dieses Kapitels ist festzuhalten, da $ß$ sich das Auftreten unvollständiger Information in Internalisierungsverhandlungen mit mehreren Beteiligten noch viel verhängnisvoller auswirkt als im bilateralen Verhandlungsmodell. Unvollständige Information liefert also neben den bekannten Standardargumenten, die sich primär auf Situationen mit vollständiger Information beziehen, einen weiteren Grund für das komplette Versagen privater Verhandlungen in "großen Gruppen". 


\section{Zwischenbilanz}

Die Zielsetzung der Kapitel 2 und 3 bestand in einer ausführlichen Untersuchung der Frage, inwieweit private Verhandlungen ein wirksames Mittel zur Internalisierung externer Effekte darstellen, wenn zwischen den Verhandlungspartnern asymmetrische Information über deren marginale Zahlungsbereitschaft für eine Ausdehnung bzw. Einschränkung des Externalitätsniveaus herrscht. Dabei stand zunächst das Bemühen im Vordergrund, einen geeigneten und plausiblen nichtkooperativen Verhandlungsproze $B$ zu modellieren, der unter den Bedingungen vollständiger Information zu einer effizienten Ressourcenallokation führte. Auf diese Weise konnte ein Defizit der traditionellen Argumentation des Coase-Theorems beseitigt werden, das darin besteht, die Pareto-Optimalität des Ergebnisses freiwilliger Internalisierungsverhandlungen, ähnlich wie in der kooperativen Verhandlungstheorie, als axiomatisch vorauszusetzen, anstatt zu zeigen, wie ein solches Ergebnis konkret zustande kommt.

Da die Annahme vollständiger Information über individuelle Zahlungsbereitschaften extrem unrealistisch ist, erschien es geboten, in dieser Hinsicht unvollständige Information in den Modellrahmen zu integrieren. Die grundlegende Struktur der Verhandlungsprozedur, insbesondere die unterstellte Fähigkeit des offerierenden Verhandlungspartners, ein ultimatives Vertragsangebot zu unterbreiten, legte es dabei nahe, den Verhandlungsprozeß als typisches Mechanism-Design-Problem aufzufassen. Dieser Ansatz ist zwar in der Literatur weit verbreitet, wirkt aber in den meisten Darstellungen technisch sehr überfrachtet. Dieser Umstand erforderte eine etwas ausführlichere, stärker auf ökonomische Inhalte bedachte Auseinandersetzung mit seinen methodischen Grundlagen.

Die Analysen des zweiten Kapitels machten deutlich, daß unvollständige Information schon im erfolgversprechendsten Fall bilateraler Verhandlungen, auf den sich die klassische Argumentation des dezentralen Lösungsansatzes schwerpunktmäßig bezieht, die Durchsetzung eines effizienten Verhandlungsergebnisses i.d.R. verhindert. Dies liegt daran, daß der Verhandlungspartner, der das Vertragsangebot formuliert, im Hinblick auf seine jeweilige Zielsetzung (Gewinn- bzw. Nutzenmaximierung) dazu gezwungen ist, dem akzeptierenden Verhandlungspartner eine sog. "Informationsrente" zu zahlen, die diesem den Anreiz zur Vorspiegelung einer falschen Zahlungsbereitschaft nehmen soll. Da diese Informationsrentenzahlung aus gesamtwirtschaftlicher Sicht keinen Kostenfaktor darstellt, weil sie lediglich eine 
Umverteilung des realisierbaren Internalisierungsgewinns zugunsten des akzeptierenden Vertragspartners darstellt, dennoch aber im individuellen Optimierungskalkül der offerierenden Partei zu Buche schlägt, wird zwischen die gesamtwirtschaftlichen und die einzelwirtschaftlichen Grenzkosten einer Ausdehnung bzw. Einschränkung des Externalitätsniveaus ein Keil getrieben, so daß es zwangsläufig zu einem allokativen Versagen der Verhandlungslösung kommen muß.

$\mathrm{Da} B$ Verhandlungen mit mehreren Beteiligten größere Probleme aufwerfen als solche zwischen zwei Individuen, erscheint intuitiv plausibel und wird auch in der Literatur betont. So schreiben z.B. Baumol und Oates (1988) unter Bezugnahme auf Buchanan (1967) über die Bedeutung privater Verhandlungen im Rahmen der Umweltökonomie folgendes: " It is generally recognized that where the number of individuals concerned is large, the likelihood of voluntary negotiation becomes small, because the administrative costs of coordination become prohibitive and because 'as the number of participants becomes critically large, the individual will more and more come to treat the behavior of all others as beyond his own possible range of influence' ". Da aber von den meisten ernsthaften Externalitätenproblemen viele Individuen betroffen seien, spiele der dezentrale Internalisierungsansatz bei umweltpolitischen Fragestellungen nur eine untergeordnete Rolle. ${ }^{21}$

In diesem Zitat klingen mit dem Transaktionskostenargument und dem Trittbrettfahrerproblem zwei der klassischen Haupteinwände gegen das Coase-Theorem an. $\mathrm{Da}$ diese beiden Phänomene ernsthafte Hindernisse für den Erfolg freiwilliger Internalisierungsverhandlungen darstellen, haben aber auch Ökonomen eingeräumt, die von ihrer Grundhaltung her mehr Vertrauen in die Kräfte des freien Marktes setzen als Baumol und Oates. Neben Buchanan gesteht nicht zuletzt Coase selbst zu: "But equally there is no reason why, on occasion, such governmental administrative regulation should not lead to an improvement in economic efficiency. This would seem particularly likely when, as is normally the case with the smoke nuisance, a large number of people are involved and in which therefore the costs of handling the problem through the market or the firm may be high."22

Diese These wird nun durch die Ergebnisse des dritten Kapitels noch gestützt. Dort hat sich gezeigt, da $B$ in einem Modellrahmen, in dem Transaktionskosten per Annahme ausgeschlossen wurden und der, was die Auswahl der Konstellation von

${ }^{21}$ Vgl. Baumol und Oates (1988, S. 10f.). Das Zitat von Buchanan stammt aus Buchanan (1967, S. 116).

22Vgl. Coase (1960, S. 18). 
Eigentumsrecht und Verhandlungsmacht betrifft, bewußt so gewählt wurde, daß bei vollständiger Information Free-Riding nicht vorkommen kann, die Informationsprobleme, die sich schon im Zwei-Personen-Fall ergeben, nun in verschärfter Form auftreten. Während bei bilateralen Verhandlungen wenigstens noch partielle Wohlfahrtsverbesserungen erzielt werden können, schwindet diese Möglichkeit bei sukzessiver Erhöhung der Anzahl der Verhandlungsteilnehmer mehr und mehr, bis im Extremfall, bei unendlich vielen Beteiligten, u.U. überhaupt keine Effizienzgewinne mehr realisierbar sind.

Obgleich in Kapitel 3 nicht alle theoretisch möglichen Konstellationen von Eigentumsordnung und Verhandlungsmacht analysiert wurden, deutet doch einiges darauf hin, daß unvollständige Information einen weiteren Grund für das völlige Scheitern freiwilliger Verhandlungen bei Externalitätsproblemen in großen Gruppen darstellt. Die Konsequenz, die sich bei Berücksichtigung des Informationsproblems für die Pigou-Coase-Kontroverse ergibt, lautet also einmal mehr: Wenn private Verhandlungen überhaupt erfolgreich sein können, dann in Fällen mit einer geringen Anzahl beteiligter Individuen. In Fällen mit vielen Beteiligten dürften dagegen kaum Zweifel daran bestehen, daß zentrale Internalisierungsmaßnahmen die bessere Alternative darstellen. Versuche, den Property-Rights-Ansatz zu retten, lohnen sich demzufolge am ehesten noch bei bilateralen Externalitätsproblemen.

Wenn man sich nunmehr auf die Betrachtung dieses Idealfalls beschränkt, wäre es etwas zu voreilig, die dezentrale Lösung allein aufgrund ihres in Kapitel 2 festgestellten allokativen Versagens zu verwerfen. Immerhin hat sich herausgestellt, $\mathrm{da} ß$ trotz der Informationsprobleme eine teilweise Internalisierung und eine Paretoverbesserung im Vergleich zum Ausgangszustand möglich ist. Was nun eine staatliche Regulierung betrifft, so müssen die Informationsprobleme, mit denen eine Zentralinstanz bei ihren Bemühungen um die Durchsetzung einer effizienten Ressourcenallokation zu kämpfen hat, qualitativ zumindest als gleichermaßen schwerwiegend, quantitativ, d.h. im Hinblick auf den Umfang der zu verarbeitenden Informationen, sogar als gravierender eingeschätzt werden. Demzufolge wäre es unzulässig, die private Verhandlungslösung an einem hypothetischen First-Best-Zustand zu messen, von dem nicht klar ist, ob er überhaupt durch eine andere Institution als den "allwissenden Planer" herbeigeführt werden kann. Man muß sich vielmehr fragen, ob für eine weniger utopische Zentralinstanz, die das Problem der Informationsbeschaffung erst lösen muß, ein besseres Allokationsergebnis erreichbar ist, als auf dem Verhandlungsweg erzielt werden kann. 
In einem ersten Schritt sollte man sich dabei überlegen, wie sich traditionelle Internalisierungsinstrumente wie Steuern und Auflagen angesichts des Informationsproblems bewähren. Im zweiten Schritt gilt es zu klären, was von elaborierten Allokationsmechanismen, die auf eine Enthüllung privater Informationen abzielen, zu halten ist. Können aus theoretischer Sicht mit Hilfe solcher Mechanismen wirklich Pareto-Verbesserungen durchgesetzt werden und können sie aus einem pragmatischen Blickwinkel ernstzunehmende Modelle einer realistischen Staatstätigkeit sein? Reicht es vielleicht schon aus, sich der bekannten Internalisierungsinstrumente zu bedienen und sie lediglich im Hinblick auf die Informationsproblematik besser abzustimmen? Diese Fragen stehen im Mittelpunkt des zweiten Teils dieser Arbeit. 


\section{Teil II}

\section{Die Internalisierung externer Effekte durch eine Zentralin- $\operatorname{stanz}$}

In diesem Teil der Arbeit sollen verschiedene Möglichkeiten diskutiert werden, die einer Zentralinstanz, z.B. der Regierung eines Landes oder einer lokalen Behörde, bei der Regulierung von Externalitäten zur Verfügung stehen. Wir wollen uns dabei auf das einfache bilaterale Externalitätenmodell mit einem Schädiger und einem Geschädigten sowie unvollständiger Information über deren Gewinn- und Schadensfunktionen beschränken. Es mag zugegebenermaßen auf den ersten Blick etwas befremdlich erscheinen, in solchen Situationen Staatseingriffe überhaupt in Erwägung zu ziehen, weil leicht der Eindruck entstehen könnte, es werde hier mit Kanonen auf Spatzen geschossen. ${ }^{1}$ Diesem potentiellen Einwand kann man die folgenden Argumente entgegenhalten:

Aus rein theoretischer Sicht ist der Hauptgegenstand der Ausführungen dieses Teils ein Effizienzvergleich zwischen privater Verhandlungslösung und staatlichen Internalisierungsmaßnahmen. Es wäre unsinnig, diesen Vergleich unter Bedingungen durchzuführen, unter denen an der Überlegenheit von Staatseingriffen ohnehin kein Zweifel besteht. $\mathrm{Da} \beta$ dies in Situationen, in denen viele Individuen von dem externen Effekt betroffen sind, sehr wahrscheinlich ist, wurde im Rahmen dieser Arbeit mehrfach begründet. Anders könnte es sich im bilateralen Fall verhalten, in dem der Property-Rights-Ansatz noch die vergleichsweise höchsten Effizienzgewinne verspricht.

Wer dennoch der Meinung ist, daß die Forderung eines Staatseingriffs bei bilateralen Externalitätsproblemen überzogen ist, weil beispielsweise die Erhebung einer Internalisierungsteuer oder die Fixierung und Überwachung einer Auflage mit hohen Transaktionskosten verbunden ist, die eine Intervention nicht lohnenswert erscheinen lassen, sollte bedenken, daß diese Probleme allein aufgrund der geringen

\footnotetext{
${ }^{1}$ Baumol und Oates (1988, S.35) kritisieren beispielsweise die Verwendung von Pigou-Steuern im "small-numbers case".
} 
Anzahl involvierter Individuen noch nicht als "Bagatellfälle" abgetan werden können.

Zum einen gibt es eine Reihe von Externalitätsbeziehungen, die zwar mehr oder weniger bilateraler Natur, dafür aber weit verbreitet sind, so daß die Wohlfahrtsverluste, die sich bei einer unzureichenden Internalisierung einstellen würden, in ihrer Summe sehr hoch sein können. Man denke in etwa an die zahlreichen Erscheinungsformen externer Effekte, die so manches gutnachbarliche Verhältnis trüben können, wie z.B. die Lärmbelästigung. Dieses Problem scheint, wie der Schutz der Nachtruhe ab 22.00 Uhr beweist, offensichtlich ernstaft genug zu sein, um es zumindest teilweise zentral zu regulieren. In Abwesenheit dieses Verbots müßte man möglicherweise mit hohen Wohlfahrtsverlusten, etwa in Form von Gerichtskosten, rechnen.

Zum anderen gibt es Externalitätenprobleme, von denen tatsächlich nur zwei Parteien betroffen sind, bei denen aber sehr viel auf dem Spiel steht. Es dürfte z.B. nicht selten vorkommen, daß von zwei benachbarten Produktionsbetrieben einer dem anderen durch seine Emissionen beträchtlichen Schaden zufügt. In einer solchen Situation wäre prinzipiell sowohl eine, wenn auch kostenträchtige, Intervention durch eine lokale Behörde, als auch eine freiwillige Eigeninitiative der beiden Firmen denkbar. Die Betrachtung des Zwei-Personen-Falls ist also auch aus empirischer Sicht nicht völlig irrelevant, wenngleich natürlich der theoretische Aspekt im Vordergrund steht. Der zweite Teil der Arbeit gliedert sich wie folgt:

In Kapitel 4 wird zunächst gezeigt, vor welchen Informationsproblemen der Staat steht, wenn er bei der Internalisierung eines externen Effekts auf traditionelle Lenkungsinstrumente wie Pigou-Steuern oder Auflagen zurückgreifen will, ohne die entscheidungsrelevanten Grenznutzen und Grenzkosten zu kennen. Dabei sollen v.a. die Anreize der Individuen, ihre Grenznutzen- bzw. Grenzkostenfunktionen falsch anzugeben, herausgearbeitet und die allokativen Konsequenzen dieses strategischen Verhaltens abgeleitet werden.

Kapitel 5 setzt sich mit den klassischen Präferenzenthüllungsmechanismen auseinander, die die in Kapitel 4 beschriebenen Informationsprobleme prinzipiell lösen könnten. Diese Mechanismen, die ursprünglich für Allokationsprobleme mit öffentlichen Gütern konzipiert wurden, werden auf das Externalitätenmodell übertragen; ihre Wirkungsweise wird kurz dargestellt, ihre wichtigsten Schwächen etwas ausführlicher diskutiert. Es wird sich herausstellen, daß es gewichtige theoretische und praktische Einwände gegen die Verwendung dieser Verfahren gibt. 
In Kapitel 6 wird auf der Grundlage der Ergebnisse des fünften Kapitels der Frage nachgegangen, wie Regulierungsmaßnahmen, bei denen die Zentralinstanz bewußt auf Informationsenthüllung verzichtet und stattdessen nur auf allgemein zugängliche Daten zurückgreift, im Vergleich zur Verhandlungslösung zu beurteilen sind. Dieser "Second-Best-Vergleich" wird zeigen, daß einfach zu handhabende Pigou-Steuern oder Auflagen, die die Wohlfahrt lediglich im Durchschnitt maximieren, der von ihrer Konzeption her viel elaborierteren und komplexeren Verhandlungslösung durchaus überlegen sein können.

\section{Informationsprobleme beim Einsatz traditioneller Lenkungsinstru- mente}

Die traditionellen staatlichen Maßnahmen zur Internalisierung externer Effekte, die sich im Laufe der Zeit aus der am Pigou-Ansatz orientierten ökonomischen Denkrichtung entwickelt haben, umfassen Auflagenlösungen, Steuern bzw. Subventionen sowie die Vergabe von Emissionszertifikaten. Die Wirkungsweise dieser Lenkungsinstrumente ist zumindest unter den Bedingungen vollständiger Information mittlerweile wohlbekannt und gehört zu den Standardthemen der Lehrbuchliteratur zur Umweltökonomie. ${ }^{2}$ Auch wenn eine Wirkungsanalyse unter diesen Idealvoraussetzungen für das Verständnis der Grundzusammenhänge unerläßlich ist, darf eine wirklich brauchbare Theorie der Internalisierung externer Effekte nicht übersehen, daß der Erfolg dieser Instrumente entscheidend davon abhängt, wie gut die regulierende Behörde über die mit der Externalität verbundenen Nutzen und Kosten informiert ist. Das allen Internalisierungsmaßnahmen gemeinsame Grundproblem besteht darin, daß die Durchsetzung eines effizienten Externalitätsniveaus ohne eben dessen Kenntnis nicht möglich ist: Die optimale Auflage ist, ebenso wie das optimale Angebot an Emissionslizenzen, identisch mit dem effizienten Externalitätsniveau, und der "richtige" Pigou-Steuersatz muß gleich dem verursachten Grenzschaden bei diesem optimalen Niveau sein. Das effiziente Externalitätsniveau ist aber nur bestimmbar, wenn die Grenznutzen- und die Grenzschadensfunktionen der Individuen bekannt sind. Da diese Informationen i.d.R. nicht öffentlich zugänglich sind, sind Versuche, mit den traditionellen Mitteln eine

\footnotetext{
${ }^{2}$ Siehe dazu beispielsweise die Lehrbücher von Baumol und Oates (1988), Pearce und Turner (1990) sowie Weimann (1990).
} 
effiziente Ressourcenallokation herbeizuführen, zum Scheitern verurteilt, wenn die Individuen kein Interesse an der Preisgabe ihrer privaten Informationen haben.

In diesem Kapitel soll gezeigt werden, welche Anreize die privaten Wirtschaftseinheiten dazu bewegen können, dem Staat eine wahrheitsgemäße Auskunft über ihre Grenznutzen- bzw. Grenzkostenfunktionen vorzuenthalten, wenn er sie einfach danach befragen und ansonsten auf die bekannten Lenkungsinstrumente vertrauen würde. Je nachdem, ob eine Auflagen- oder eine Steuerlösung angekündigt wird, werden die Individuen ihren Grenznutzen bzw. ihre Grenzkosten auf unterschiedliche Weise falsch angeben, wenn dies ihren ökonomischen Zielsetzungen förderlich ist. ${ }^{3}$ Solche strategischen Falschangaben, die bei der optimalen Bereitstellung öffentlicher Güter zu den Hauptproblemen zählen, scheinen im Zusammenhang mit externen Effekten eher vernachlässigt worden zu sein. ${ }^{4}$ Es erscheint deshalb sinnvoll, zunächst die allokativen Konsequenzen herauszuarbeiten, mit denen man zu rechnen hätte, wenn man auf die Ehrlichkeit der Wirtschaftssubjekte vertrauen würde. Schließlich sollte man die simple Fragemethode erst verwerfen, wenn klar ist, daß und weshalb sie zum Scheitern verurteilt ist.

Als Grundlage für die folgenden Ausführungen soll im wesentlichen das in Kapitel 1 beschriebene einfache Externalitätenmodell mit einem Schädiger und einem Geschädigten dienen. Es wird angenommen, daß der Staat weder die Grenzgewinnfunktion $b^{\prime}(x)$ noch die Grenzschadensfunktion $d^{\prime}(x)$ kennt. Er weiß lediglich, da $b^{\prime}(x)$ streng monoton fällt, $d^{\prime}(x)$ monoton steigt und daß die beiden Kurven nur innerhalb gewisser allgemein bekannter Bandbreiten liegen können. Das Ausmaß einer eventuellen Über- oder Untertreibung kann sich also nur innerhalb bestimmter Grenzen bewegen, deren Überschreitung unglaubwürdig wäre. Die Frage ist nun, wie sich die Individuen in dieser Ausgangssituation verhalten, wenn man sie nach ihrer Grenzgewinn- bzw. Grenzschadensfunktion fragt und sie wissen, welches Instrument (Auflage oder Steuer) die zentrale Behörde einsetzen will.

\footnotetext{
${ }^{3} \mathrm{Da}$ in diesem Kapitel aus den genannten Gründen nur mit einem Schädiger und einem Geschädigten argumentiert wird, scheidet eine Regulierung mittels Emissionszertifikaten von vornherein aus, weil kein Konkurrenzmarkt für diese Zertifikate zustande kommen kann. Selbst wenn man zuließe, daß auch der Geschädigte Lizenzen aufkaufen kann, um das Aktivitätsniveau des Schädigers zu begrenzen, wäre der dadurch erreichte Wettbewerbsgrad nicht ausreichend. ${ }^{4}$ Vgl. aber Kwerel (1977).
} 


\subsection{Strategisches Verhalten des Geschädigten bei Auflagen- und Abgabenlö- sung}

Beginnen wir mit dem Verhalten des Geschädigten, das besonders einfach zu beschreiben ist, und betrachten dazu die folgende Abbildung.

\section{Abbildung 14}

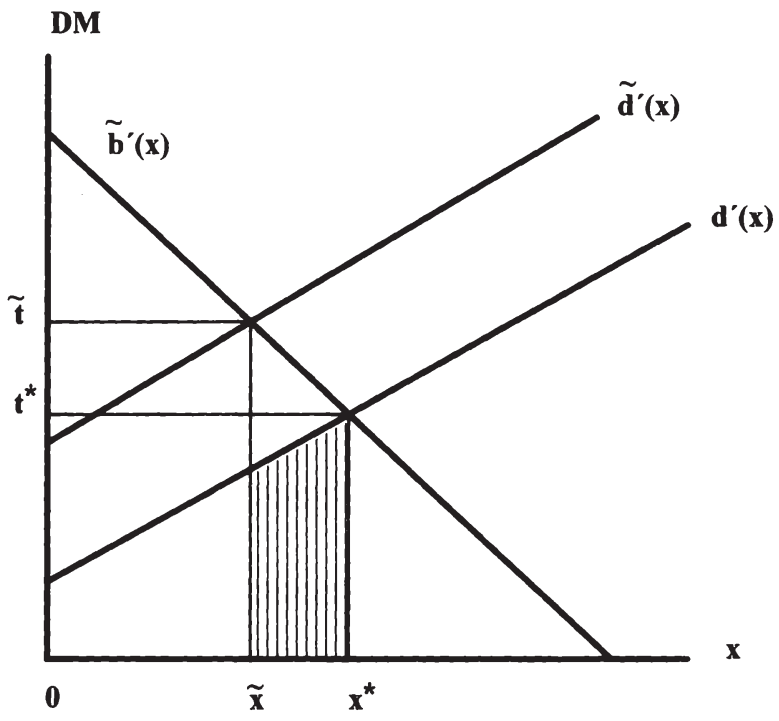

Die Kurve $d^{\prime}(x)$ bezeichne die wahre Grenzschadensfunktion des Geschädigten, $\tilde{b}^{\prime}(\mathrm{x})$ stehe für eine beliebige, möglicherweise auch falsch bekundete, Grenzgewinnfunktion des Schädigers. Die Frage ist nun, welche Grenzschadensfunktion der Geschädigte bei gegebenem $\tilde{b}^{\prime}(x)$ dem Staat nennen würde, wenn er danach gefragt würde. Bei wahrheitsgemäßer Bekundung von $\mathrm{d}^{\prime}(\mathrm{x})$ müßte er damit rechnen, da $\beta$ die Zentralinstanz bei einer Auflagenlösung die Produktionsmenge $\mathrm{x}^{*}$ als Emissionsstandard vorschreibt oder bei einer Steuerlösung die Produktion des Schädigers mit dem Satz t* pro Mengeneinheit besteuert. In beiden Fällen wäre sein zu erwartendes Schadensniveau $\mathrm{d}\left(\mathrm{x}^{*}\right)$, in Abbildung 14 dargestellt durch die Fläche 
unterhalb der Kurve $d^{\prime}(x)$ in den Grenzen von 0 bis $x^{*}$. Da der Geschädigte versuchen würde, den Schaden durch seine Angaben möglichst gering zu halten, ist klar, da $B$ er unabhängig vom angekündigten Instrument und unabhängig von der vom Schädiger bekundeten Grenzgewinnfunktion seine Grenzschadensfunktion bis an die Grenze der Glaubwürdigkeit übertreiben würde. M.a.W. ist die Angabe des höchstmöglichen Grenzschadens für ihn sowohl bei der Auflagen- als auch bei der Steuerlösung dominante Strategie.

Wenn wir annehmen, daß die Kurve $\tilde{d}^{\prime}(x)$ in Abbildung 14 den höchstmöglichen marginalen Schaden beschreibt, dann wäre das Ergebnis dieser hypothetischen Befragung, das im übrigen natürlich noch von $\tilde{b}^{\prime}(x)$ abhängt, das Produktionsniveau $\tilde{\mathrm{x}}$. Dieses Produktionsniveau würde sich entweder einstellen, weil es direkt vorgeschrieben würde oder weil es beim Steuersatz $\tilde{\mathfrak{t}}$, den der Staat unter diesen Umständen wählen würde, dem Gewinnmaximum des Schädigers entspräche. Der Schaden, den der Geschädigte durch diese Falschangabe vermeiden würde, entspräche der schraffierten Fläche in Abbildung 14.

Würde sich also die regulierende Behörde die benötigten Informationen über die Grenzschadenskurve durch eine solche Form der Befragung beschaffen, wäre das implementierte Externalitätsniveau bei isolierter Betrachtung des Verhaltens des Geschädigten zu niedrig. Welches $\mathrm{x}$ sich insgesamt einstellt, hängt aber auch davon ab, welche Angaben der Schädiger über seine marginalen Gewinne macht.

\subsection{Strategisches Verhalten des Schädigers}

Das Verhalten des Schädigers bei diesem "naiven" Befragungsmodus differiert je nachdem, welches Instrument die Zentralbehörde ankündigt.

\subsection{1 Übertreibung des Grenzgewinns bei der Auflagenlösung}

Bei einer Auflagenlösung hat der Schädiger ein Interesse an einer lockeren Regelung, die ihm die Realisation seines isolierten Gewinnmaximums $\overline{\mathrm{x}}\left(\mathrm{mit} \mathrm{b}^{\prime}(\overline{\mathrm{x}})\right.$ $=0$ ) nach wie vor ermöglicht. Dies erreicht er, wie in Abbildung 15 dargestellt, durch eine geeignete Übertreibung seiner Grenzgewinnfunktion. 


\section{Abbildung 15}

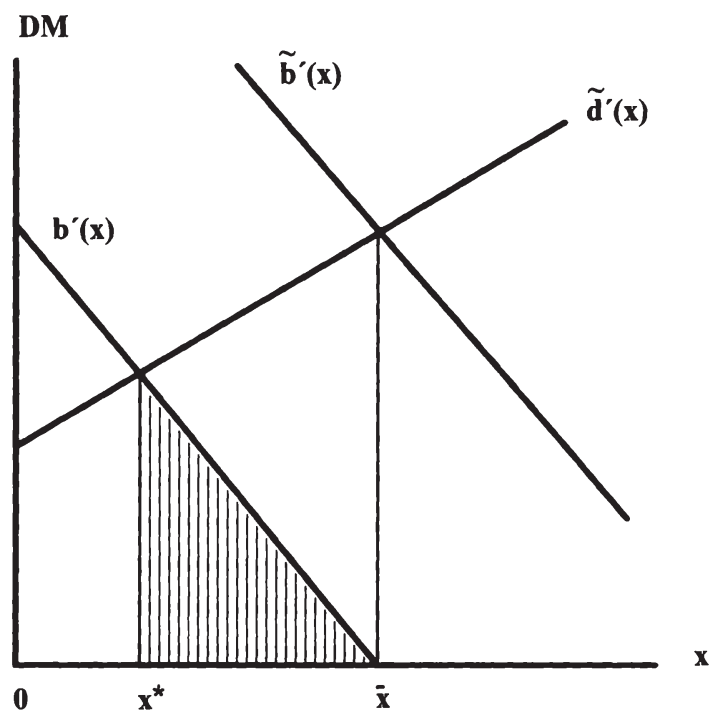

Dabei wird er als rationales Individuum antizipieren, daß der Geschädigte in jedem Fall die höchstmögliche Grenzschadensfunktion $\tilde{d}^{\prime}(x)$ angeben wird. Würde er unter dieser Bedingung seine wahre Grenzgewinnfunktion $b^{\prime}(x)$ offenbaren, würde der Standard bei $x^{*}$ fixiert werden. Durch die Angabe von $\tilde{b}^{\prime}(x)$ könnte er dagegen die Auflage $\bar{x}$ durchsetzen, die gerade seinem persönlichen Gewinnmaximum entspräche. Die Angabe von $\tilde{b}^{\prime}(x)$ ist demnach beste Antwort auf die dominante Strategie des Schädigers, $\tilde{d}^{\prime}(x)$ anzugeben. Damit bildet dieses Strategiepaar ein Nash-Gleichgewicht, in dem das isolierte Gewinnmaximum $\overline{\mathrm{x}}$ des Schädigers als vermeintliches Wohlfahrtsoptimum implementiert wird. Dieses Nash-Gleichgewicht ist eindeutig. Falls $\tilde{b}^{\prime}(x)$ nicht innerhalb der Bandbreite glaubwürdiger Grenzgewinnfunktionen liegt, ist die beste Antwort des Schädigers die Angabe der maximal möglichen Schadensfunktion und die gleichgewichtige Allokation kleiner als $\overline{\mathbf{x}}$. 


\subsubsection{Untertreibung des Grenzgewinns bei der Abgabenlösung}

Das Verhalten des Schädigers bei einer Steuerlösung bedarf einer etwas genaueren Betrachtung. Klar ist, daß eine Übertreibung des Grenzgewinns nun nicht mehr in Frage kommt, weil dadurch der Pigou-Steuersatz ansteigen würde, so daß sich der Schädiger im Vergleich zu einer wahrheitsgemäßen Offenbarung schlechter stellen würde. Nur eine Untertreibung kann in diesem Fall eine sinnvolle Strategie sein. Ausmaß und Wirkung dieser Untertreibung auf das implementierte Externalitätsniveau gilt es im folgenden zu bestimmen.

\section{Abbildung 16}

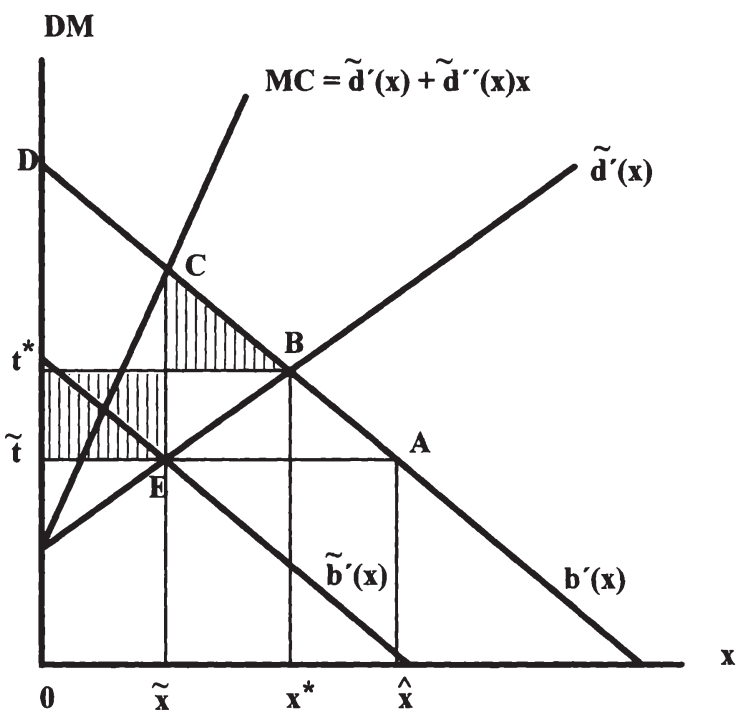

Es scheint auf den ersten Blick plausibel zu sein, daß der Schädiger bei Anwendung einer Pigou-Steuer seinen marginalen Gewinn so stark wie möglich untertreiben wird, um die Zentralbehörde dazu zu bewegen, einen niedrigen Steuersatz zu wählen und somit die privaten Grenzkosten einer Erhöhung seines Produktionsniveaus zu minimieren. Bei diesem falschen Steuersatz würde er dann entsprechend 
seiner tatsächlichen Gewinnfunktion einen Output wählen, der höher ist als der, den die Zentralinstanz in Anbetracht der erhaltenen Informationen eigentlich durchsetzen wollte. Diese Möglichkeit ist in Abbildung 16 dargestellt.

Wenn $\tilde{b}^{\prime}(x)$ eine beliebige Grenzgewinnkurve bezeichnet, die der Schädiger angeben kann, ohne unglaubwürdig zu erscheinen, könnte er zunächst den Steuersatz bis auf $\tilde{\mathfrak{t}}$ drücken, dann aber den gemäß seiner wahren Grenzgewinnkurve $b^{\prime}(x)$ optimalen Output $\hat{\mathbf{x}}$ mit $b^{\prime}(\hat{\mathbf{x}})=\tilde{\mathfrak{t}}$ produzieren. Falls diese Rechnung aufginge, könnte er gegenüber der korrekten Angabe von $b^{\prime}(x)$, die den Steuersatz t* zur Folge hätte, einen Zusatzgewinn erzielen, der in Abbildung 16 der Fläche t* $\tilde{\mathrm{t}} \mathrm{AB}$ entspricht. Es ist jedoch aus folgendem Grund nicht zu erwarten, daß diese Strategie den erwünschten Erfolg hat: Wenn der Schädiger beim Steuersatz $\tilde{\mathrm{t}}$ die Menge $\hat{x}$ herstellen würde, so würde er der regulierenden Behörde damit signalisieren, daß seine Angaben zur Grenzgewinnfunktion nicht der Wahrheit entsprochen haben. Bei den hier unterstellten Kurvenverläufen könnte die Behörde anhand der Produktionsentscheidung auf die wahre Grenzgewinnfunktion schließen, den Steuersatz künftig auf $t^{*}$ anheben und dem Schädiger nachträglich eine Geldbuße auferlegen. Durch eine solche Geldbuße, beispielsweise in Höhe des durch die Täuschung erlangten Zusatzgewinns, könnten die Vorteile der Falschangabe vollständig neutralisiert werden.

Wenn der Schädiger derartige Strafmaßnahmen antizipiert, kann er ihnen dadurch begegnen, $\mathrm{da} \beta$ er sich so verhält, als habe er tatsächlich die angegebene Grenzgewinnfunktion $\tilde{b}^{\prime}(\mathrm{x})$. Dazu müßte er die Menge $\tilde{\mathbf{x}}$ in Abbildung 16 produzieren. Die Zentralbehörde könnte ihm in diesem Fall nicht beweisen, die Unwahrheit gesagt zu haben, selbst wenn sie seine Strategie durchschaut. Auf diese Weise könnte er zumindest den niedrigen Steuersatz $\tilde{\mathfrak{t}}$ durchsetzen, wenngleich er sich mit einer Beschränkung seines Outputs zufrieden geben müßte. $\mathrm{Da} ß$ sich dieses Verhalten bei geeigneter Untertreibung des Grenzgewinns dennoch auszahlen würde, ist aus Abbildung 16 ersichtlich: Hätte der Schädiger die Wahrheit gesagt, so hätte er nach Steuern einen Gewinn in Höhe des Inhalts der Fläche $t^{*} B D$ erzielen können. Demgegenüber steht ein Nettogewinn in Höhe des Inhalts der Fläche t ECD bei Angabe der falschen Grenzgewinnkurve. Welche Strategie vorteilhafter ist, hängt davon ab, ob die schraffierte Rechtecksfläche kleiner oder größer ist als die schraffierte Dreiecksfläche. Hier ist offensichtlich die Untertreibung die bessere Wahl. Das folgende Kalkül zeigt, daß eine Untertreibung bis zu einem gewissen Punkt immer besser ist, als die wahrheitsgemäße Offenbarung: 
Der Schädiger wird das Ausmaß der Untertreibung so wählen, daß der Output $\tilde{\mathbf{x}}$, den er einhalten muß, um seine Angaben glaubwürdig erscheinen zu lassen, den Gewinn

$$
\pi(\tilde{\mathbf{x}})=b(\tilde{\mathbf{x}})-\mathrm{t}(\tilde{\mathrm{x}}) \tilde{\mathrm{x}}
$$

maximiert. Da der Staat den Pigou-Steuersatz $\mathrm{t}(\tilde{\mathrm{x}})=\tilde{\mathrm{d}}^{\prime}(\tilde{\mathrm{x}})$ setzen wird und $\tilde{\mathrm{d}}^{\prime \prime}(\mathrm{x})>$ 0 ist, muß der Schädiger berücksichtigen, da $\beta$ eine Steigerung des Outputs um eine Einheit seine Produktionskosten in zweifacher Hinsicht erhöht: Zum einen steigt die Steuerlast bei konstantem Steuersatz um $\tilde{\mathrm{d}}^{\prime}(\tilde{\mathbf{x}})$, zum anderen wird der Steuersatz selbst in die Höhe getrieben. Dieser Effekt ist mit der Gesamtproduktion zu multiplizieren, so daß sich eine zusätzliche Belastung in Höhe von $\tilde{\mathrm{d}}$ "' $(\tilde{\mathrm{x}}) \tilde{\mathrm{x}}$ ergibt. Die notwendige Bedingung für das optimale Produktionsniveau $\tilde{\mathrm{x}}$ lautet demnach

$$
b^{\prime}(\tilde{x})=t(\tilde{x})+t^{\prime}(\tilde{x}) \tilde{x}=\tilde{d}^{\prime}(\tilde{x})+\tilde{d}^{\prime \prime}(\tilde{x}) \tilde{x}
$$

Die Kurve $\mathrm{MC}=\tilde{\mathrm{d}}^{\prime}(\mathrm{x})+\tilde{\mathrm{d}}^{\prime \prime}(\mathrm{x}) \mathrm{x}$, die die marginale Veränderung der Steuerlast beschreibt, liegt wegen $\tilde{\mathrm{d}}^{\prime \prime}(\mathrm{x})>0$ oberhalb der angegebenen Grenzschadenskurve $\tilde{\mathrm{d}}^{\prime}(\mathrm{x})$. Falls $\tilde{\mathrm{d}}^{\prime}(\mathrm{x})$ wie in Abbildung 16 linear verläuft, halbiert MC den Winkel, der von Ordinate und Grenzschadenskurve eingeschlossen wird. Das optimale Produktionsniveau liegt dort, wo MC und $\mathrm{b}^{\prime}(\mathrm{x})$ sich schneiden. Da an dieser Stelle $\mathrm{b}^{\prime}(\tilde{\mathrm{x}})>\tilde{\mathrm{d}}^{\prime}(\tilde{\mathrm{x}})$ ist, ist $\tilde{\mathrm{x}}$ kleiner als das Produktionsniveau $\mathrm{x}^{*}$, das sich bei wahrheitsgemäßer Offenbarung eingestellt hätte. Um sein persönliches Optimum durchzusetzen, muß der Schädiger dem Staat die Grenzgewinnkurve $\tilde{b}^{\prime}(x)$ nennen, die die Grenzschadenskurve $\tilde{\mathrm{d}}^{\prime}(\mathrm{x})$ bei $\tilde{\mathbf{x}}$ schneidet.

Das Nash-Gleichgewicht bei der Steuerlösung ist also dadurch charakterisiert, $\mathrm{da} ß$ der Schädiger seine Grenzgewinnfunktion wie soeben beschrieben untertreibt ${ }^{5}$, während der Geschädigte seine Schadensfunktion wieder so stark wie möglich übertreibt. Beide Strategien führen zur Implementation einer Produktionsmenge, die im Vergleich zum Paretooptimum zu niedrig ist.

Es sollte noch darauf hingewiesen werden, daß der Anreiz, Falschangaben zu machen, auf Seiten des Schädigers verschwindet, wenn dieser davon ausgeht, den Steuersatz nicht beeinflussen zu können. In diesem Fall wäre aus seiner Sicht $\tilde{d}^{\text {"' }}(\mathbf{x})$

\footnotetext{
${ }^{5}$ Natürlich ist auch hier wieder eine Randlösung möglich, wenn das optimale Ausmaß der Untertreibung unglaubwürdig wirken würde.
} 
$=0$ und die Optimalitätsbedingung (4.2) würde $z u b^{\prime}(x)=\tilde{d}^{\prime}(x)$, d.h. es wäre für ihn optimal, $\mathrm{x}^{*}$ zu produzieren. Dazu müßte er aber keine Falschangabe machen. Damit stellt sich außerhalb des hier untersuchten Szenarios die Frage, inwieweit in Fällen mit sehr vielen Schädigern, die den Steuersatz als gegeben betrachten, überhaupt ein Informationsproblem besteht. Tatsache ist, da $\mathrm{B}$ es in dieser Situation aus der Sicht des einzelnen Schädigers keine Rolle spielt, welche Grenzgewinnkurve er angibt, da er durch sein isoliertes Handeln den Steuersatz ohnehin nicht verändern könnte. Gegeben das Verhalten seiner Opponenten könnte er also genauso gut die Wahrheit sagen. Ein positiver Anreiz, den Grenzgewinn zu untertreiben, besteht aber im Rahmen dieser nicht-kooperativen Betrachtungsweise nicht. Dies wäre anders, wenn sich die Individuen kooperativ verhalten und sich gemeinsam auf eine Strategie der Untertreibung festlegen würden. Unter dieser Voraussetzung könnten sie den Steuersatz drücken und sich alle gegenüber einer wahrheitsgemäßen Offenbarung verbessern. In diesem Fall würden sie den Steursatz aber von vornherein nicht mehr als gegeben betrachten.

Vor dem Hintergrund dieser Überlegungen weist die Literatur über Informationsenthüllungsverfahren bei Externalitätsproblemen in "großen Gruppen" eine Inkonsistenz auf. Dies zeigt sich beispielsweise in einer Arbeit von Sinn (1993), in der ein einfaches Informationsenthüllungsverfahren vorgeschlagen wird, bei dem u.a. angenommen wird, daß die (vielen) Verursacher den Pigou-Steuersatz als gegeben betrachten. Das Problem an diesem Mechanismus scheint mir das folgende zu sein: Einerseits ist nicht klar, ob das Verfahren überhaupt nötig ist, wenn "Preisnehmerverhalten" vorliegt, da die Möglichkeit, den Steuersatz zu manipulieren, den eigentlichen Anreiz bietet, Falschangaben zu machen. Andererseits kann man zeigen, daß Sinns Mechanismus seine Enthüllungseigenschaft verliert, wenn die Annahme des Preisnehmerverhaltens fallengelassen wird. Diese Inkonsistenz hätte m.E. vermieden werden müssen. ${ }^{6}$

Wenn man die Ergebnisse dieses kurzen Kapitels zusammenfaßt, muß man feststellen, daß die Ausgestaltung von Internalisierungsmaßnahmen auf der Basis von Informationen, die auf dem Wege einer einfachen Befragung der beteiligten Individuen gewonnen wurden, $\mathrm{zu}$ ineffizienten Allokationen führt. Bei einer

${ }^{6}$ Eine ähnliche Inkonsistenz weisen auch ältere Arbeiten, wie z.B. die von Roberts und Spence (1976) oder Kwerel (1977) auf, die Enthüllungsverfahren u.a. unter Verwendung von Zertifikaten vorschlagen. Die kritische Annahme ist hier Preisnehmerverhalten auf dem Markt für Zertifikate. 
Auflagenlösung ist trotz des Anreizes des Geschädigten, seinen Grenzschaden zu übertreiben, ein ineffizient hohes Aktivitätsniveau zu erwarten, wenn der Schädiger seinen Grenzgewinn hinreichend stark übertreiben kann, ohne unglaubwürdig zu erscheinen. Das Aktivitätsniveau ist in diesem Fall mit dem isolierten Gewinnmaximum des Schädigers identisch. Ist die Glaubwürdigkeitsrestriktion dagegen bindend, kann das Aktivitätsniveau kleiner, größer oder auch zufällig gleich der pareto-optimalen Allokation sein.

Bei einer Steuerlösung ist unter allen Umständen mit einem ineffizient niedrigen Aktivitätsniveau zu rechnen. Dafür spricht sowohl der Anreiz des Geschädigten, seinen marginalen Schaden zu übertreiben, als auch das Bestreben des Schädigers, den Pigou-Steuersatz durch eine Untertreibung des Grenzgewinns zu drücken. Dabei wurde davon ausgegangen, daß letzterer seine falschen Angaben durch eine entsprechende Produktionsentscheidung bestätigen muß, um eventuellen Strafmaßnahmen des Staates zu entgehen. Dieser Zwang, den Betrug zu kaschieren, führte letztendlich zur eindeutigen Unterschreitung des pareto-optimalen Aktivitätsniveaus.

Wie nicht anders zu erwarten, können mit Hilfe dieser denkbaren aber naiven Methode der Informationsgewinnung keine vernünftigen Allokationsergebnisse erzielt werden. Daraus leitet sich der Bedarf an Mechanismen ab, die besser dazu geeignet sind, an die benötigten Daten heranzukommen. Die Diskussion solcher Mechanismen wird Gegenstand des folgenden Kapitels sein. 


\section{Mechanismen zur Präferenzenthüllung}

In Kapitel 4 wurde gezeigt, daß die Internalisierung externer Effekte mit Hilfe des klassischen Instrumentariums erhebliche Informationsprobleme aufwirft: Je nachdem, ob eine Auflage oder eine Pigou-Steuer zum Einsatz kommen soll, haben die Individuen unterschiedliche Anreize, ihre "Präferenzen" für die Externalität zu verschleiern, wenn sie danach befragt werden. Würde die zentrale Behörde an dieser naiven Befragungsmethode festhalten, wäre es ihr nicht möglich, die wahren Grenznutzen bzw. Grenzkosten in Erfahrung zu bringen. Die Folge wären die oben beschriebenen Allokationsstörungen. Wenn die Behörde das pareto-optimale Externalitätsniveau durchsetzen will, muß sie also auf elaboriertere Allokationsmechanismen zurückgreifen, die die Anreize, private Informationen zu verhüllen, beseitigen.

$\mathrm{Da} ß$ dabei im Prinzip die gleichen Mechanismen angewendet werden können, die im Zusammenhang mit der Enthüllung von Präferenzen für öffentliche Güter bekannt geworden sind, erscheint naheliegend. Der nächste logische Schritt in der Argumentation besteht also in der Übertragung dieser Mechanismen auf unser Externalitätsproblem. Dieser Schritt soll im folgenden unternommen werden, wobei es angesichts der Fülle von Literaturbeiträgen, die sich mit Präferenzenthüllungsmechanismen befassen, notwendig ist, sich auf die wichtigsten Aspekte zu beschränken. Es wird schwerpunktmäßig darum gehen, die Schwächen dieser Verfahren hervorzuheben und zu begründen, warum sie nicht oder nur bedingt dazu geeignet sind, die bestehenden Informationsprobleme in befriedigender Weise zu lösen.

\subsection{Wahrheitsgemäße Offenbarung als dominante Strategie: Die Klasse der Groves-Mechanismen}

Die ersten Ansätze zur Überwindung von Informationsproblemen, die bei der Bereitstellung öffentlicher Güter durch den Staat auftreten, stammen überwiegend aus den frühen siebziger Jahren. Als bekannteste Literaturstellen sind hier Clarke (1971) und Groves (1973) sowie die Pionierarbeit von Vickrey (1961), zu nennen. Das Hauptanliegen dieser Arbeiten bestand in der Entwicklung direkter Enthüllungsmechanismen mit der Eigenschaft, daß für die Nutznießer die korrekte Offenbarung ihrer Präferenzen für das öffentliche Gut dominante Strategie und die Im- 
plementation eines effizienten Bereitstellungsniveaus möglich sein sollte. Ein direkter Enthüllungsmechanismus ist dadurch charakterisiert, daß die Beteiligten aufgefordert sind, ihre Präferenzen unmittelbar anzugeben. Die Konzentration auf solche Mechanismen kann wieder durch das Enthüllungsprinzip gerechtfertigt werden. Für Mechanismen mit dominanten Strategien besagt dieses Theorem folgendes:

Jedes Ergebnis, das durch einen beliebigen Mechanismus als Gleichgewicht in dominanten Strategien implementierbar ist, kann auch durch einen direkten Enthüllungsmechanismus implementiert werden, in dem die wahrheitsgemäße Angabe der privaten Informationen ein Gleichgewicht in dominanten Strategien bildet. ${ }^{1}$

Das Enthüllungsprinzip impliziert, daß man mit einem indirekten Mechanismus, in dem den Agenten beliebige andere Strategien als die Angabe von Präferenzen zur Verfügung stehen, keine anderen Ergebnisse erzielen kann, als mit einem direkten Mechanismus. Die Suche nach indirekten Mechanismen mit dominanten Strategien, die ebenfalls zur Implementation effizienter Allokationen führen, erübrigt sich damit.

Auf dieser Grundlage kann man sich nun fragen, welche Vorteile ein Mechanismus bietet, bei dem die Offenbarung der wahren Präferenzen dominante Strategie ist. Diese Eigenschaft liegt dann vor, wenn sich kein Individuum durch die Vorspiegelung falscher Präferenzen Vorteile verschaffen kann, gleich welche Präferenzen die anderen Individuen bekunden. Die Vorzüge solcher Mechanismen liegen auf der Hand: Ein Spieler, der über eine dominante Strategie verfügt, braucht keinerlei Vermutungen über das mögliche Verhalten seiner Gegenspieler anzustellen. Er muß sich keine Gedanken darüber machen, ob die anderen Individuen ihre Präferenzen richtig oder falsch angeben, ja er braucht nicht einmal die möglichen Typen von Präferenzen und ihre statistische Verteilung zu kennen. Ein Mechanismus mit dominanten Strategien stellt also keine allzu hohen Ansprüche an die Fähigkeiten der Spieler, strategisch zu denken, und senkt damit die Gefahr versehentlichen Fehlverhaltens auf ein Mindestmaß. Darüber hinaus wird das Problem multipler Gleichgewichte weitgehend ausgeschaltet, es sei denn, manche Spieler sind indifferent ge-

\footnotetext{
${ }^{1}$ Auf den Beweis dieses Theorems wird wieder verzichtet. Die grundlegende Struktur ist weitgehend identisch mit der des Beweises auf Seite $37 \mathrm{f}$. Als Literaturstelle ist wieder Dasgupta, Hammond und Maskin (1979, S. 194 f.) oder auch Muller und Satterthwaite (1985, S.135 f.) zu nennen.
} 
genüber mehreren dominanten Strategien. ${ }^{2}$ Ein Mechanismus mit dominanten Strategien bietet der regulierenden Behörde also ein hohes $\mathrm{Ma} B$ an Sicherheit darüber, $\mathrm{da} B$ sich das von ihr angestrebte Gleichgewicht auch tatsächlich einstellt.

Wenn man sich ausschließlich auf die Klasse direkter Enthüllungsmechanismen mit dominanten Strategien beschränkt, gelangt man zwangsläufig zu den sogenannten "Groves-Mechanismen". Wie Green und Laffont (1977) und (1979) gezeigt haben, sind die Mechanismen der Groves-Klasse die einzigen, die diese Merkmale aufweisen, so daß sich die Suche nach Alternativen erübrigt. ${ }^{3}$ Wie diese Mechanismen, angewandt auf unser Externalitätsproblem, funktionieren, soll im folgenden beschrieben werden.

\subsubsection{Charakterisierung der Groves-Mechanismen}

Für eine Charakterisierung der Groves-Mechanismen ist es zweckmäßig, wenigstens ansatzweise zu modellieren, in welcher Weise private Information in die Gewinnfunktion des Schädigers und in die Schadensfunktion des Geschädigten eingeht. Es wird angenommen, daß die Grenzgewinnfunktion, abgesehen von der Produktionsmenge $\mathrm{x}$, noch von einem Informationsparameter a abhängt, also durch $b^{\prime}(x, a)$ gegeben ist. Ebenso soll die Grenzschadensfunktion von einem Informationsparameter $\theta$ abhängen, so daß sie durch eine Funktion $\mathrm{d}^{\prime}(\mathrm{x}, \theta)$ beschrieben werden kann. Man kann sich beispielsweise vorstellen, daß diese Funktionen linear sind und ihre Achsenabschnitte und/oder ihre Steigungen mit dem jeweiligen Informationsparameter variieren. Auf die genauen Abhängigkeiten kommt es aber gegenwärtig nicht an.

Es wird nun davon ausgegangen, daß eine zentrale Behörde das effiziente Niveau der Externalität implementieren will, wobei sie zwar weiß, von welchem Funktionstyp $\mathrm{b}^{\prime}\left(\right.$.) und $\mathrm{d}^{\prime}($.$) sind, die konkreten Ausprägungen von a und \theta$ aber nicht beobachten kann. Um an die benötigte Information heranzukommen, bedient sie sich des folgenden Verfahrens: Sie fragt Schädiger und Geschädigten nach ihren Informati-

\footnotetext{
${ }^{2}$ Ein Beispiel für einen Mechanismus mit mehreren dominanten Strategien findet man in Dasgupta, Hammond und Maskin (1979, S.195).

$3 \mathrm{Vgl}$. Green und Laffont (1977) bzw. (1979, S. 56 ff.). Green und Laffont (1979) ist wohl nach wie vor eines der Standardwerke über Enthüllungsmechanismen und bildet die Hauptgrundlage der Ausführungen dieses Kapitels. Die umfassende Darstellung, die dieses Buch bietet, ist an manchen Stellen allerdings sehr abstrakt, ökonomisch oft wenig einleuchtend und daher mühsam zu lesen.
} 
onsparametern, nachdem sie angekündigt hat, sie werde dem Schädiger diejenige Produktionsmenge als Auflage vorschreiben, bei der sein angeblicher Grenzgewinn mit dem angeblichen Grenzschaden des Geschädigten übereinstimmt. Die beiden können sich also ausrechnen, daß das erlaubte Produktionsniveau auf $x^{*}(\hat{a}, \hat{\theta})$ mit

$$
b^{\prime}\left(x^{*}(\hat{a}, \hat{\theta}), \hat{a}\right)=d^{\prime}\left(x^{*}(\hat{a}, \hat{\theta}), \hat{\theta}\right)
$$

beschränkt wird, wenn die Parameter â bzw. $\hat{\theta}$ angegeben werden.

Weiterhin gibt die Zentralinstanz bekannt, sie werde dem Geschädigten einen Transfer

$$
t_{G}=b\left(x^{*}(\hat{a}, \hat{\theta}), \hat{a}\right)+T_{G}(\hat{a})
$$

und dem Schädiger einen Transfer

$$
\mathrm{t}_{\mathrm{s}}=-\mathrm{d}\left(\mathrm{x}^{*}(\hat{\mathrm{a}}, \hat{\theta}), \hat{\theta}\right)+\mathrm{T}_{\mathrm{S}}(\hat{\theta})
$$

zahlen. Diese Transfers setzen sich aus je einer Anreiz- und einer Lump-SumKomponente zusammen. Die beiden Anreizkomponenten $b\left(x^{*}(\hat{a}, \hat{\theta}), \hat{a}\right)$ beziehungsweise - $d\left(x^{*}(\hat{a}, \hat{\theta}), \hat{\theta}\right)$, die in Abwesenheit von Einkommenseffekten ohne Probleme aus der Grenzgewinnfunktion b'(.) bzw. aus der Grenzschadensfunktion $d^{\prime}($.$) berechnet werden können, haben den Zweck, die Individuen zur wahrheitsge-$ mäßen Offenbarung ihrer privaten Informationen zu bewegen. $\mathrm{Da}$ und wie sie ihren Zweck erfüllen, wird in Kürze gezeigt werden. Die beiden Lump-Sum-Komponenten $T_{G}(\hat{a})$ und $T_{S}(\hat{\theta})$, bezüglich der sich verschiedene Mechanismen der GrovesKlasse unterscheiden, können im Prinzip beliebig gestaltet werden. Sie könnten beispielsweise dazu verwendet werden, das Budget der Zentralinstanz, die den Mechanismus koordiniert, auszugleichen. Wichtig ist nur, daß sie nicht vom Informationsparameter des jeweiligen Transferempfängers abhängen dürfen, da sonst die Wirkung der Anreizkomponenten zerstört würde. Natürlich können die $t_{i}$ je nach Höhe der $T_{i}$ auch negativ sein.

Die Behauptung ist nun, daß bei Anwendung dieses Mechanismus die Angabe des wahren Informationsparameters für beide Individuen dominante Strategie ist und das effiziente Externalitätsniveau implementiert wird. Zur Überprüfung dieser 
Behauptung müssen die Nutzenpositionen der beiden Individuen unter Berücksichtigung der angekündigten Transferzahlungen ermittelt werden.

Falls der wahre Informationsparameter des Geschädigten $\theta$ ist und er $\hat{\theta}$ signalisiert, ist sein Nutzen durch

$$
\mathrm{u}(\hat{\mathrm{a}}, \hat{\theta}, \theta)=\mathrm{t}_{\mathrm{G}}(\hat{\mathrm{a}}, \hat{\theta})-\mathrm{d}\left(\mathrm{x}^{*}(\hat{\mathrm{a}}, \hat{\theta}), \theta\right)=\mathrm{b}\left(\mathrm{x}^{*}(\hat{\mathrm{a}}, \hat{\theta}), \hat{\mathrm{a}}\right)-\mathrm{d}\left(\mathrm{x}^{*}(\hat{\mathrm{a}}, \hat{\theta}), \theta\right)+\mathrm{T}_{\mathrm{G}}(\hat{a})
$$

bestimmt, wenn der Schädiger den Informationsparameter â nennt. Dessen Gewinn ist gegeben durch

$$
\pi(\hat{a}, \hat{\theta}, a)=b\left(x^{*}(\hat{a}, \hat{\theta}), a\right)+t_{s}(\hat{a}, \hat{\theta})=b\left(x^{*}(\hat{a}, \hat{\theta}), a\right)-d\left(x^{*}(\hat{a}, \hat{\theta}), \hat{\theta}\right)+T_{S}(\hat{\theta})
$$

wenn a sein tatsächlicher Informationsparameter ist und er â angibt, während der Geschädigte $\hat{\theta}$ signalisiert.

Aus den Gleichungen (5.4) und (5.5) ist unmittelbar ersichtlich, daß es für die Individuen immer optimal ist, ihre privaten Informationen wahrheitsgemäß zu offenbaren. Betrachten wir beispielsweise das Kalkül des Geschädigten und nehmen an, der Schädiger signalisiere einen beliebigen Informationsparameter $\hat{a}$. Würde der Geschädigte lügen und $\hat{\theta} \neq \theta$ angeben, dann würde die Zentralinstanz von einer gesamtwirtschaftlichen Wohlfahrt in Höhe von $b(x, \hat{a})-d(x, \hat{\theta})$ ausgehen und die Produktionsmenge $x^{*}(\hat{a}, \hat{\theta})$ wählen, die diesen Ausdruck maximiert. Würde der Geschädigte dagegen der Wahrheit entsprechend $\hat{\theta}=\theta$ nennen, dann würde die Zentralinstanz den Output auf die Menge $x^{*}(\hat{a}, \theta)$ begrenzen, die die Differenz $b(x, \hat{a})$ $\mathrm{d}(\mathrm{x}, \theta)$ maximiert. Diese Differenz ist aber bis auf die Konstante $\mathrm{T}_{\mathrm{G}}$ identisch mit der Zielfunktion des Geschädigten, d.h. die Zentralinstanz maximiert den Nutzen des Geschädigten dann und nur dann, wenn er die Wahrheit sagt. Andernfalls wählt sie einen Produktionsstandard, der im Hinblick auf seine Zielsetzung suboptimal ist. Es gilt nämlich für alle $\hat{a}, \hat{\theta}$ und $\theta$ per Definition von $x^{*}(\hat{a}, \theta)$ und $x^{*}(\hat{a}, \hat{\theta})$

$$
b\left(x^{*}(\hat{a}, \theta), \hat{a}\right)-d\left(x^{*}(\hat{a}, \theta), \theta\right) \geq b\left(x^{*}(\hat{a}, \hat{\theta}), \hat{a}\right)-d\left(x^{*}(\hat{a}, \hat{\theta}), \theta\right)
$$

Insbesondere gilt diese Ungleichung unabhängig vom Signal des Schädigers, d.h. die Angabe des wahren Informationsparameters ist für den Geschädigten in der Tat dominante Strategie. 
Die gleiche Argumentation gilt natürlich analog für den Schädiger, so daß die wahrheitsgemäße Offenbarung der privaten Information durch beide Individuen ein Nash-Gleichgewicht in dominanten Strategien bildet. Auf der Basis dieser Information ist es der Zentralinstanz möglich, diejenige Auflage festzulegen, die die tatsächliche Wohlfahrt maximiert. Groves-Mechanismen gewährleisten demnach die Enthüllung privater Information in dominanten Strategien und eine effiziente Ressourcenallokation, womit obige Behauptung bewiesen ist.

\subsubsection{Schwächen der Groves-Mechanismen}

Würde man sich mit den im vorangegangenen Abschnitt beschriebenen Eigenschaften von Groves-Mechanismen zufrieden geben, könnte die Diskussion "Zentrale versus dezentrale Internalisierung externer Effekte bei unvollständiger Information" an dieser Stelle beendet werden. Die Schlußfolgerung aus den bisherigen Erkenntnissen müßte lauten: Private Verhandlungen führen in Gegenwart unvollständiger Information zu Wohlfahrtsverlusten, während eine Zentralinstanz mit den Groves-Mechanismen über Instrumente verfügt, mit deren Hilfe die bestehenden Informationsprobleme gelöst und First-Best-Allokationen realisiert werden können. Demzufolge sollte man die Internalisierung externer Effekte besser dem Staat überlassen. Diese Bilanz schon an dieser Stelle zu ziehen wäre etwas verfrüht, da Groves-Mechanismen mit verschiedenen Mängeln behaftet sind, die ihre Brauchbarkeit bei näherer Betrachtung stark relativieren. Die wichtigsten dieser Mängel sollen im folgenden diskutiert werden. ${ }^{4}$

\subsubsection{Versagen in Gegenwart von Einkommenseffekten}

Ein Mangel besteht in der Tatsache, daß Groves-Mechanismen ihre Anreizwirkungen i.a. verlieren, wenn in den Präferenzordnungen der Individuen, anders als bisher unterstellt, Einkommenseffekte in bezug auf die Externalität auftreten. So geben beispielsweise Green und Laffont (1977 u. 1979) verschiedene Beispiele an, in denen kein Präferenzenthüllungsmechanismus mit dominanten Strategien existiert,

\footnotetext{
${ }^{4}$ Vgl. dazu auch den Überblick von Groves und Ledyard (1977a).
} 
weil Einkommenseffekte vorhanden sind. ${ }^{5}$ Diese Beispiele, auf die hier nicht näher eingegangen werden soll, sind nicht weiter erstaunlich, wenn man sich auf einen wichtigen Satz aus der "Social-Choice-Theory" besinnt. Dieser Satz, der auf zwei unabhängig voneinander entstandene Arbeiten von Gibbard (1973) und Satterthwaite (1975) zurückgeht und dementsprechend als "Gibbard-Satterthwaite-Theorem" bekannt wurde, besagt folgendes:

Es existiert keine gesellschaftliche Entscheidungsregel, die

a) auf uneingeschränkte individuelle Präferenzordnungen anwendbar,

b) nicht-diktatorisch und

c) nicht durch die Angabe falscher Präferenzen manipulierbar ist, weil die Angabe der wahren Präferenzen für alle Gesellschaftsmitglieder dominante Strategie ist. ${ }^{6}$

Eine gesellschaftliche Entscheidungsregel ist eine Funktionsvorschrift, die den individuellen Präferenzen der Gesellschaftmitglieder einen "besten sozialen $\mathrm{Zu}$ stand" aus einer Menge realisierbarer Alternativen zuordnet. ${ }^{7}$ Ein sozialer Zustand kann beispielsweise eine bestimmte Ressourcenallokation sein.

Man bezeichnet eine gesellschaftliche Entscheidungsregel als diktatorisch, wenn sie für jede denkbare Konstellation individueller Präferenzordnungen einen sozialen Zustand als beste Alternative auswählt, der identisch ist mit dem von einem einzelnen Individuum, dem "Diktator", am meisten präferierten Zustand aus der Al-

\footnotetext{
${ }^{5}$ Vgl. Green und Laffont (1977, S. 437 bzw. 1979, S.79ff.).

6 Eine einführende Darstellung inclusive einer einfachen Skizzierung der Beweisidee des GibbardSatterthwaite-Theorems findet man bei Feldman (1982, S. 196 ff.).

${ }^{7}$ Das Konzept einer "gesellschaftlichen Entscheidungsregel" ist vom Konzept der "sozialen Wohlfahrtsfunktion" zu unterscheiden: Während erstere individuellen Präferenzordnungen eine beste Alternative zuweist, aggregiert letztere die individuellen Präferenzen zu einer gesellschaftlichen Präferenzordnung. (Vgl. dazu Feldman (1982, S. 196 f.)). Man beachte deshalb die Unterschiede zwischen dem Gibbard-Satterthwaite-Theorem und Arrows Unmöglichkeitstheorem: Arrow (1951) ging es darum, bei bekannten individuellen Präferenzen die Unmöglichkeit der Existenz einer sozialen Wohlfahrtsfunktion zu beweisen, die die von ihm postulierten Axiome erfüllt. Das Gibbard-Satterthwaite-Theorem bezieht sich demgegenüber auf die Nicht-Existenz gesellschafilicher Entscheidungsregeln mit bestimmten Eigenschaften bei unbekannten Präferenzen. Zwischen beiden Theoremen besteht allerdings ein enger Zusammenhang, der von Satterthwaite (1975) herausgearbeitet wurde.
} 
ternativenmenge. ${ }^{8}$ Eine solche Entscheidungsregel ignoriert also die Präferenzen aller Gesellschaftsmitglieder mit Ausnahme der des Diktators und ist damit höchst undemokratisch.

Der Berührungspunkt zwischen dem Gibbard-Satterthwaite-Theorem und den Groves-Mechanismen besteht darin, daß letztere nichts anderes sind, als nicht-diktatorische gesellschaftliche Entscheidungsregeln: Sie ordnen der Grenzgewinnfunktion des Schädigers und der Grenzschadensfunktion des Geschädigten (deren "Präferenzen"), ein Externalitätsniveau und bestimmte Transferzahlungen (einen "sozialen Zustand") zu. Dabei kommt es sehr wohl auf die Präferenzen beider Individuen an, da das resultierende Externalitätsniveau eine Kompromißlösung darstellt, die weder vom Schädiger noch vom Geschädigten favorisiert wird. Ersterem wäre es natürlich am liebsten, wenn er sein isoliertes Gewinnmaximum realisieren könnte, während letzterer ein vollständiges Verbot der Ausübung des externen Effekts bevorzugen würde. Die Tatsache, daß der Mechanismus keine dieser Extremlösungen generiert, zeigt, daß er nicht-diktatorisch ist.

Gleichzeitig ist im Rahmen eines Groves-Mechanismus aber auch die Angabe der wahren Präferenzen für alle Individuen dominante Strategie. Nach den Aussagen des Gibbard-Satterthwaite-Theorems können aber beide Eigenschaften bei uneingeschränkten individuellen Präferenzordnungen nicht gleichzeitig erfüllt sein. D.h. umgekehrt: Die Eigenschaft dominanter Strategien kann allenfalls dann vorliegen, wenn man spezielle Präferenzordnungen betrachtet. Wie wir gesehen haben, stellt die Beschränkung auf quasi-lineare Präferenzen offensichtlich eine hinreichende Bedingung für die Existenz eines derartigen Mechanismus dar. ${ }^{9}$

Die Frage ist nun, wie diese Einschränkung zu bewerten ist. Allgemein ist die Annahme quasi-linearer Präferenzen natürlich unrealistisch, insbesondere dann, wenn es sich bei den Wirtschaftssubjekten nicht um Unternehmen, sondern um private Haushalte, wie sie in der Mikroökonomie betrachtet werden, handelt. In diesem Fall ist nicht zu erwarten, daß der Grenznutzen bzw. der Grenzschaden unabhängig von der Höhe des Einkommens ist. Was den hier angestrebten Vergleich zwischen zentralem und dezentralem Internalisierungsansatz betrifft, ist die Annahme aber nur von untergeordneter Bedeutung, da sie auch bei der Herleitung der Verhandlungslösung getroffen wurde. Zudem ist keineswegs klar, ob die dortige Vorge-

${ }^{8}$ Vgl. dazu beispielsweise Bossert und Stehling (1990, S.84 f.).

${ }^{9} \mathrm{Vgl}$. dazu auch Kreps (1990, S. 712 f.). 
hensweise noch möglich ist, wenn man allgemeinere Präferenzen zuläßt. ${ }^{10}$ Aus diesem Grund wird die Rolle von Einkommenseffekten, wenn auch nicht ohne grundsätzliche Bedenken, im folgenden ausgeblendet.

\subsubsection{Die Unmöglichkeit eines ausgeglichenen Budgets}

Ein anderer Mangel, der den Groves-Mechanismen anhaftet und der im Hinblick auf die zentrale Fragestellung dieser Arbeit als wesentlich problematischer zu beurteilen ist, beruht auf der Tatsache, daß das Budget der Zentralinstanz, die die Transfer- bzw. Steuerzahlungen festlegt, um die richtigen Anreize zur Präferenzenthüllung zu setzen, i.a. nicht ausgeglichen ist.

Das Defizit D erhält man durch Addition der Gleichungen (5.2) und (5.3). Im Enthüllungsgleichgewicht eines Groves-Mechanismus, also bei Bekundung der wahren Präferenzen, lautet die Bedingung für einen ausgeglichenen Haushalt

$$
D=t_{G}+t_{S}=\left[b\left(x^{*}(a, \theta), a\right)-d\left(x^{*}(a, \theta), \theta\right)\right]+T_{G}(a)+T_{S}(\theta)=0 .
$$

Der Ausdruck in den eckigen Klammern entspricht gerade dem gesamtwirtschaftlichen Überschuß, der bei Anwendung eines Groves-Mechanismus realisiert wird. Dieser Überschuß soll im folgenden mit $S(a, \theta)$ bezeichnet werden. Wenn man eine strikte allgemeine Gleichgewichtsbetrachtung anstellt und den Zufluß externer Mittel ausschließt, kann ein Budgetausgleich nur dadurch erreicht werden, daß die fehlenden Mittel in Höhe des gesamtwirtschaftlichen Überschusses von Schädiger und Geschädigtem gemeinsam aufgebracht werden. Dies könnte nur durch geeignete Wahl der Lump-Sum-Komponenten $\mathrm{T}_{\mathrm{G}}(\mathrm{a})$ und $\mathrm{T}_{\mathrm{S}}(\theta)$ bewerkstelligt werden. Wenn man die Budgetgleichung (5.7) in der Form

$$
S(a, \theta)=-\left[T_{G}(a)+T_{S}(\theta)\right]
$$

\footnotetext{
${ }^{10}$ Die Annahme quasi-linearer Präferenzen war entscheidend bei der Herleitung von Gleichung (2.15) auf Seite 47. Durch den expliziten Zusammenhang zwischen Output- und Transferfunktion, der durch diese Gleichung hergestellt wurde, ergab sich eine starke Vereinfachung des Optimierungskalküls des Schädigers. Wie dieses Kalkül ohne diese Vereinfachung gelöst werden kann, ist unklar.
} 
schreibt, so ist klar, daß der Haushalt unter Beibehaltung des Lump-Sum-Charakters von $T_{G}$ und $T_{S}$ für beliebige $a$ und $\theta$ nur dann ausgeglichen sein kann, wenn der Überschuß $\mathrm{S}(\mathrm{a}, \theta)$ additiv separabel in a und $\theta$ ist. Die additive Separabilität ist aber eine sehr spezielle Eigenschaft, die ohne restriktive Zusatzannahmen kaum zu erwarten ist. Um dies zu zeigen, genügt es, ein Gegenbeispiel anzugeben.

Betrachten wir dazu die spezielle Version des einfachen Externalitätenmodells, die in Abschnitt 2.5 zur Veranschaulichung der Verhandlungslösung herangezogen wurde.

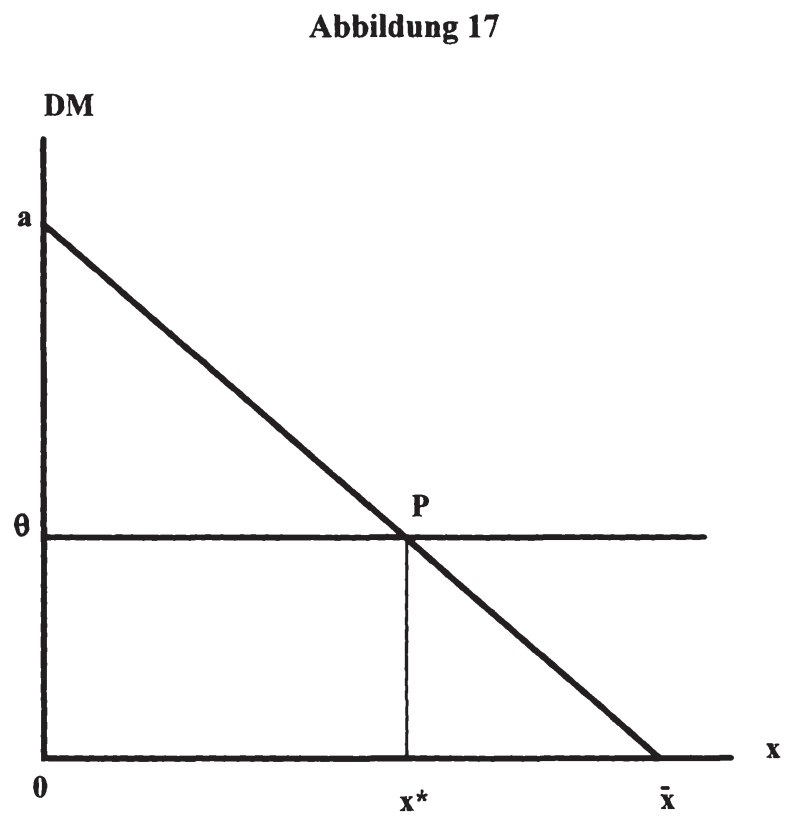

Die Grenzgewinnfunktion des Schädigers war dort durch

(5.9) $b^{\prime}(x, a)=a-c x$,

die Grenzschadensfunktion des Geschädigten durch

(5.10) $\mathrm{d}^{\prime}(\mathrm{x}, \theta)=\theta$ 
gegeben, wobei a und $\theta$ die privaten Informationsparameter darstellen. Bei Anwendung eines Groves-Mechanismus werden die Individuen ihre privaten Informationen enthüllen, so $\mathrm{da} \beta$ das effiziente Externalitätsniveau $x^{*}(a, \theta)$ aus

(5.11) $\mathrm{a}-\mathrm{cx}=\theta$

berechnet werden kann. Man erhält

(5.12) $\mathrm{x}^{*}(\mathrm{a}, \theta)=(\mathrm{a}-\theta) / \mathrm{c}$.

Der gesamtwirtschaftliche Überschuß $S(a, \theta)$, der als Summe der Anreizkomponenten des Transferschemas bei jedem beliebigen Groves-Mechanismus in der Budgetgleichung der Zentralinstanz auftaucht, ergibt sich einfach nach Berechnung der Dreiecksfläche $\theta \mathrm{Pa}$ in Abbildung 17. Man erhält

$$
\mathrm{S}(\mathrm{a}, \theta)=(1 / 2) \mathrm{x}^{*}(\mathrm{a}-\theta)=(\mathrm{a}-\theta)^{2} / 2 \mathrm{c}=\left(\mathrm{a}^{2}+\theta^{2}-2 \mathrm{a} \theta\right) / 2 \mathrm{c}
$$

Da dieser Ausdruck offensichtlich nicht additiv separabel bezüglich a und $\theta$ ist, ist es in diesem Beispiel unmöglich, Funktionen $\mathrm{T}_{\mathrm{G}}(\mathrm{a})$ und $\mathrm{T}_{\mathrm{S}}(\theta)$ zu finden, die Gleichung (5.8) für beliebige Werte von a und $\theta$ erfüllen."

Welche Konsequenzen hat nun die Unmöglichkeit eines Budgetausgleichs für die Anwendbarkeit von Groves-Mechanismen? Diese Frage kann am besten am Beispiel des Clarke-Mechanismus als dem bekanntesten Spezialfall eines Groves-Mechanismus diskutiert werden. Dieses Präferenzenthüllungsverfahren, das ursprünglich zur Lösung des Informationsproblems bei der Bereitstellung öffentlicher Güter entwickelt wurde, kann ohne weiteres auf unser Externalitätenproblem übertragen werden.

Die Grundidee des Clarke-Mechanismus beruht auf der Erkenntnis, daß die Individuen durch ihre Präferenzangaben bei gesellschaftlichen Entscheidungsprozessen der Allgemeinheit insofern Kosten aufbürden, als bei diesen Entscheidungen meist Kompromisse geschlossen werden müssen, die alle Gesellschaftsmitglieder zum Verzicht auf die Verwirklichung ihrer Idealvorstellungen zwingen. Um zu verhindern, daß die Individuen die Kompromißlösung durch Falschangaben zu ihren Gunsten verzerren, sollten ihnen diese sozialen Kosten als eine Art "Internali-

${ }^{11}$ Weitere Beispiele findet man in Green und Laffont (1979, S. $90 \mathrm{ff}$.). 
sierungssteuer" angelastet werden. Diese Idee führt zur folgenden speziellen Ausgestaltung des Mechanismus:

Jedem der beiden Individuen wird eine sog. Clarke-Steuer in Höhe des Nutzenverlustes auferlegt, den es beim anderen durch die Bekundung seiner Präferenzen angeblich verursacht. Wenn der Schädiger beispielsweise den Informationsparameter â signalisiert, geht die Zentralinstanz davon aus, daß seine Grenzgewinnfunktion $b^{\prime}(x, \hat{a})$ ist und er seinen gewinnmaximalen Output $\bar{x}(\hat{a})$ mit $b^{\prime}(\bar{x}(\hat{a}), \hat{a})=$ 0 produzieren würde, wenn er auf den Geschädigten keine Rücksicht zu nehmen hätte. Sein angeblicher Gewinn wäre in diesem Fall gleich $b(\bar{x}(\hat{a}), \hat{a})$. Nennt der Geschädigte den Informationsparamerter $\hat{\theta}$, so rechnet die Zentralinstanz mit der Grenzschadensfunktion $d^{\prime}(x, \hat{\theta})$ und beschränkt das Produktionsniveau des Schädigers gemäß Optimalitätsbedingung (5.1) auf $x^{*}(\hat{a}, \hat{\theta})$. Entsprechend der Gewinnfunktion, die der Schädiger signalisiert hat, beschert ihm dieser Kompromiß eine Gewinneinbuße in Höhe von $b(\bar{x}(\hat{a}), \hat{a})-b\left(x^{*}(\hat{a}, \hat{\theta}), \hat{a}\right)$. Diesen Betrag hat der Geschädigte als Steuer an die Zentralinstanz abzuführen. In Anlehnung an Gleichung (5.2) gilt also beim Clarke-Mechanismus speziell

$$
t_{G}=-\left[b(\bar{x}(\hat{a}), \hat{a})-b\left(x^{*}(\hat{a}, \hat{\theta}), \hat{a}\right)\right]
$$

Der Nutzenverlust, den der Geschädigte durch die Präferenzbekundung des Schädigers erleidet, entspricht seinen angeblichen Schäden bei Implementation von $\mathrm{x}^{*}(\hat{\mathrm{a}}, \hat{\theta})$. Die Clarke-Steuer des Schädigers ist also durch $\mathrm{d}\left(\mathrm{x}^{*}(\hat{\mathrm{a}}, \hat{\theta}), \hat{\theta}\right)$ bestimmt, so $\mathrm{da} \beta$

$(5.3)^{\prime} \mathrm{t}_{\mathrm{s}}=-\mathrm{d}\left(\mathrm{x}^{*}(\hat{\mathrm{a}}, \hat{\theta}), \hat{\theta}\right)$

gilt.

Ein Vergleich zwischen (5.2) und (5.2)' bzw. (5.3) und (5.3)' zeigt, daß es sich beim Clarke-Mechanismus um ein spezielles Mitglied der Groves-Klasse mit $\mathrm{T}_{\mathrm{G}}(\hat{\mathrm{a}})=-\mathrm{b}(\overline{\mathrm{x}}(\hat{\mathrm{a}}), \hat{\mathrm{a}})$ und $\mathrm{T}_{\mathrm{S}}(\hat{\theta})=0$ handelt. Aus diesem Grund führt auch dieser Mechanismus zur Offenbarung der wahren Informationsparameter a und $\theta$.

Das gesamte Aufkommen der Clarke-Steuer erhält man durch Addition von (5.2)' und (5.3)', so daß sich im Enthüllungsgleichgewicht ein Haushaltsüberschuß in Höhe von 


$$
\begin{aligned}
& -D=-\left(t_{G}+t_{S}\right)=b(\bar{x}(a), a)-\left[b\left(x^{*}(a, \theta), a\right)-d\left(x^{*}(a, \theta), \theta\right)\right]= \\
& =b(\bar{x}(a), a)-S(a, \theta)
\end{aligned}
$$

ergibt. In Abbildung 17 entspricht das Steueraufkommen dem Inhalt der Fläche $0 \overline{\mathrm{x}} \mathrm{P}$ $\theta$, wobei die Rechtecksfläche $0 x^{*} \mathrm{P} \theta$ den Anteil des Schädigers und die Dreiecksfläche $x^{*} \bar{x} P$ den Anteil des Geschädigten repräsentiert.

Die Frage ist nun, wie dieser Budgetüberschuß verwendet werden soll. Wenn man eine allgemeine Gleichgewichtsbetrachtung anstellt, dann $m u ß$ der Haushalt der Zentralinstanz ausgeglichen sein. Die einzige Möglichkeit, im abgeschlossenen Modell einen Budgetausgleich herbeizuführen, ist der Rücktransfer an die Steuerzahler. Dabei würde aber folgendes Problem auftreten: Nehmen wir an, das Steueraufkommen werde proportional nach einem bestimmten Schlüssel aufgeteilt und den Individuen zurückerstattet. Wenn diese die Rückzahlung antizipieren, würden sie sie von vornherein in ihren Optimierungskalkülen berücksichtigen. Da jedes Individuum seine eigene private Information kennt und bestimmte Vermutungen über die private Information des anderen Individuums haben wird, könnte es sich zumindest einen subjektiven Erwartungswert des gesamten Steueraufkommens und seines Anteils daran ausrechnen. Dieser Anteil würde aber i.a. von seinem eigenen Informationsparameter abhängen, es sei denn, der Haushaltsüberschuß könnte so aufgeteilt werden, daß der Erstattungsbetrag des Geschädigten nicht von $\theta$ und der des Schädigers nicht von a abhängt. Dies ist aber, wie aus Gleichung (5.14) ersichtlich ist, nur möglich, wenn der gesamtwirtschaftliche Überschuß $S(a, \theta)$ additiv separabel bezüglich a und $\theta$ ist. $\mathrm{Da} \beta$ dies i.a. nicht der Fall sein wird, hat das obige Beispiel gezeigt. Eine Rückerstattung der Clarke-Steuer hätte also i.d.R. keinen Lump-Sum-Charakter und würde die urprünglich beabsichtigten Anreizwirkungen zerstören.

Da eine Wiederausschüttung nicht in Frage kommt, wenn auf die Enthüllungseigenschaft des Mechanismus nicht verzichtet werden soll, müßte die Zentralinstanz die Mittel einfach einbehalten und sie der Nutzung durch die Individuen entziehen. Da die Zentralinstanz aber nur als neutrales Organ zu betrachten ist, das den Interessenkonflikt zwischen Schädiger und Geschädigtem wohlwollend zu lösen hat, selbst aber keinen Nutzen aus den Ressourcen ziehen kann, unterscheidet sich diese Lösung im Grunde genommen kaum von dem etwas überspitzt formulierten Vorschlag von Kreps (1990): "It is probably safest to burn any surplus."12 Im ge-

${ }^{12}$ Vgl. Kreps (1990, S. 711). 
schlossenen Modell muß die Clarke-Steuer demnach als Ressourcenverzehr abgeschrieben werden, der bei ihrer Anwendung als unvermeidlich hinzunehmen ist.

Unter diesen Umständen kann das Verfahren aber nicht mehr als effizient bezeichnet werden. Es gewährleistet lediglich die Enthüllung der entscheidungsrelevanten Informationen und ermöglicht dadurch die Implementation desjenigen Externalitätsniveaus, bei dem Grenzgewinn und Grenzschaden übereinstimmen. Gleichzeitig zieht es aber eine Art "Doppelbelastung" der Individuen nach sich: Obwohl der Schädiger Anreizsteuern in Höhe des verursachten Schadens bezahlt, muß der Geschädigte diesen Schaden selbst tragen, weil die Steuermittel nicht dazu verwendet werden dürfen, ihn zu kompensieren. Die Schadenskosten fallen also aus gesamtwirtschaftlicher Sicht zweimal an. Das gleiche gilt für die Gewinneinbußen, die der Schädiger aufgrund des zwangsweisen Verzichts auf die Realisation seines Gewinnmaximums hinnehmen muß. Auswege aus diesem Dilemma können nur gefunden werden, wenn man den engen Rahmen des geschlossenen Modells sprengt und zuläßt, daß der Haushaltsüberschuß "nach außen" abfließen kann.

Eine naheliegende Verwendungsmöglichkeit des Clarke-Steuer-Aufkommens wäre unter diesen Umständen der Abbau verzerrender Steuern, die ansonsten zur Finanzierung öffentlicher Ausgaben erhoben werden müßten. Ein solcher Steuerabbau würde aber im Prinzip die Anreize der Individuen, ihre Präferenzen richtig anzugeben, genauso zunichte machen wie ein direkter Rücktransfer. Wenn die Wirtschaftssubjekte die "Steuerreform" antizipieren, so rechnen sie in ihren Nutzenmaximierungskalkülen mit einer Reduzierung der steuerlichen Zusatzlasten, die letztendlich wieder von den angegebenen Informationsparametern abhängig ist. Dadurch wird die Anreizstruktur des Clarke-Mechanismus ebenfalls zerstört.

Als letzte Alternative bliebe nur noch die Verteilung des Steueraufkommens an Dritte, die vom Externalitätsproblem gar nicht betroffen und demzufolge am Clarke-Mechanismus nicht beteiligt sind. Man könnte dabei z.B. an eine Spende zugunsten Bedürftiger denken. Aber auch diese Variante ist aus folgendem Grund kritisch zu beurteilen: Falls die Individuen, die die Anreizsteuer zu zahlen haben, gewisse altruistische Motive gegenüber den Transferempfängern hegen, sind wieder die oben beschriebenen Rückkopplungseffekte zu erwarten, weil die "Freude am Geben" den Spendern Nutzen stiftet, den sie in ihren Kalkülen berücksichtigen werden. Fehlen dagegen derartige Motive, ist der Ressourcenentzug als reine Zwangsmaßnahme zu bewerten und kommt aus der Sicht der Steuerzahler einer "Verbrennung" der überschüssigen Mittel recht nahe. 
Wenn man nun berücksichtigt, daß die Clarke-Steuer eigentlich keinen anderen Zweck hat, als die Wohlfahrt der Individuen zu erhöhen, die vom betrachteten Externalitätsproblem betroffen sind, so muß man sich fragen, ob es unter Gerechtigkeitsaspekten zu vertreten ist, den "Gesamtkuchen", über den die Beteiligten prinzipiell verfügen könnten, zu maximieren, sie aber gleichzeitig dazu zu zwingen, Teile davon an Außenstehende abzugeben, die vom ursprünglichen Allokationsproblem gar nicht berührt sind. Diese Frage stellt sich umso mehr, als mit der privaten Verhandlungslösung eine Alternative zur Verfügung steht, die für die Betroffenen insgesamt möglicherweise geringere Nutzeneinbußen mit sich bringt als die ClarkeSteuer. Daß eine solche Konstellation durchaus auftreten kann, zeigt ein einfaches Rechenbeispiel, das sich wieder auf das vorher betrachtete spezielle Modell bezieht.

Der Wohlfahrtsverlust, der sich in diesem Modell bei der Verhandlungslösung aufgrund der Abweichung der vom Schädiger gewählten Produktionsmenge vom First-Best-Output ergab, errechnete sich in Abschnitt 2.5 für eine gegebene Realisation des Grenzschadens $\theta$, unabhängig vom Achsenabschnitt a der Grenzgewinnfunktion, zu

$$
v(\theta)=\theta^{2} / 2 c .^{13}
$$

Das Clarke-Steuer-Aufkommen für eine bestimmte Kombination von a und $\theta$ erhält man nach Berechnung des Inhalts der Fläche $0 \bar{x} P \theta$ in Abbildung 17. Es ergibt sich

$$
T_{c}(a, \theta)=\theta x^{*}(a, \theta)+(\theta / 2)\left[\bar{x}(a)-x^{*}(a, \theta)\right]=\left(2 a \theta-\theta^{2}\right) / 2 c
$$

Der Wohlfahrtsverlust, der bei der Verhandlungslösung auftritt, ist demzufolge genau dann kleiner als der Wohlfahrtsverlust, der den Individuen durch die ClarkeSteuer entsteht, wenn a $>\theta$ gilt. Dies ist aber durch die Annahmen in Abschnitt 2.5, die zur Vermeidung von Randlösungen getroffen wurden, in jedem Fall gesichert.14

Würde man nun in einer solchen Situation die betroffenen Individuen fairerweise selbst über den institutionellen Rahmen, innerhalb dessen der externe Effekt internalisiert werden soll, entscheiden lassen, so ist zu vermuten, daß beide für die dezentrale Lösung votieren werden: Der Geschädigte würde in jedem Fall die Verhandlungslösung bevorzugen, da er hier Zahlungen zu erwarten hätte, während er

${ }^{13} \mathrm{Vgl.} \mathrm{S.} 56$ oben.

${ }^{14} \mathrm{Vgl}$. S. 55 unten. 
beim Clarke-Mechanismus nicht einmal Kompensationszahlungen für seine erlittenen Schäden erhalten würde und darüber hinaus die Anreizsteuer zahlen müßte. Der Schädiger wäre zwar im Clarke-Mechanismus besser gestellt als bei der Verhandlungslösung, da die Anreizsteuer, die er zu entrichten hätte, im Gleichgewicht immer den tatsächlichen Nutzeneinbußen des Geschädigten entspräche. Seine Gewinnposition wäre also identisch mit der, die er im Verhandlungsmodell bei vollständiger Information erreichen würde, weil er sich die Informationsrente spart. Es wäre aber durchaus denkbar, daß der Geschädigte den Schädiger mit Hilfe von Seitenzahlungen dazu bringt, ebenfalls für die dezentrale Lösung zu stimmen. Aber selbst dann, wenn ein solches Arrangement mißlingt, sollte in diesem speziellen Fall m.E. die private Verhandlungslösung als gerechter beurteilt werden, weil eine Regulierung mit Hilfe des Clarke-Mechanismus einerseits die externen Transferempfänger in ungerechtfertigter Weise begünstigen würde, während andererseits der Geschädigte extrem benachteiligt wäre. In jedem Fall kann die Clarke-Steuer im Vergleich zur Verhandlungslösung nicht als pareto-besser bezeichnet werden.

Die vorangegangene Diskussion sollte deutlich gemacht haben, daß der auf den ersten Blick scheinbar so perfekte Clarke-Mechanismus bei genauerer Betrachtung doch erhebliche Schwächen aufweist. Insbesondere das Problem des unausgeglichenen Budgets zieht unangenehme Konsequenzen nach sich. Bei einer strikten allgemeinen Gleichgewichtsanalyse im abgeschlossenen Modell muß das Aufkommen der Clarke-Steuer als Ressourcenverzehr abgeschrieben werden, weshalb der Mechanismus nicht als effizient bezeichnet werden kann. Wenn man zuläßt, daß das Steueraufkommen "modellexternen" Verwendungen zufließt, sind zwei Fälle zu unterscheiden: Entweder treten Rückkopplungseffekte auf, weil die Verwendung der Anreizsteuern ihren Zahlern indirekt doch wieder zugute kommt. In diesem Fall ist zu erwarten, daß die Anreizstrukturen des Mechanismus genauso beeinträchtigt werden wie bei einem direkten Rücktransfer. Wird das Steueraufkommen dagegen unbeteiligten Dritten zur Verfügung gestellt, treten Verteilungswirkungen auf, die zu einer Verletzung des Pareto-Kriteriums führen können und deshalb, gemessen an der Zielsetzung des Mechanismus, problematisch erscheinen. ${ }^{15}$ Diese Aussagen gelten im Prinzip für jeden Groves-Mechanismus, der einen Budgetüberschuß erzeugt.

\footnotetext{
15In der Literatur wird im Zusammenhang mit der Unmöglichkeit einer anreizneutralen Rückerstattung der Clarke-Steuer meist von Effizienzproblemen gesprochen, während Verteilungsargumente praktisch keine Rolle spielen. Angesichts der Möglickeit eines Transfers an Dritte kann die Clarke-Steuer aber strenggenommen nicht als ineffizient bezeichnet werden. Sie ist allerdings i.a. nicht dazu geeignet, Pareto-Verbesserungen durchzusetzen.
} 
Auf spezielle Mechanismen, die Haushaltsdefizite verursachen, wurde nicht explizit eingegangen. Es sind aber hier die gleichen Schwierigkeiten mit umgekehrten Vorzeichen zu erwarten. Zusammenfassend kann also festgestellt werden, daß GrovesMechanismen nicht in der Lage sind, die Informationsprobleme, die bei der Internalisierung externer Effekte auftreten, in befriedigender Weise zu lösen.

\subsubsection{Versuche zur Rettung der Groves-Mechanismen}

Die Schwierigkeiten, die die Unmöglichkeit eines Budgetausgleichs bei der Anwendung von Groves-Mechanismen bereitet, erscheinen nach den Ausführungen des vorangegangenen Abschnitts als zu schwerwiegend, um sie als Randprobleme abzutun. Wenn man sie vermeiden will, muß man gezwungenermaßen Abstriche in Bezug auf die ansonsten wünschenswerten Eigenschaften dieser Mechanismen in Kauf nehmen und sich mit Alternativen begnügen, die u.U. weniger stark ausgeprägte Anreize zur Präferenzenthüllung bieten. Diese Konsequenz könnte allenfalls dann vermieden werden, wenn man zeigen könnte, daß die entstehenden Haushaltsdefizite bzw. -überschüsse "hinreichend klein" sind, so daß die Rückkopplungseffekte einer zusätzlichen Besteuerung bzw. Steuerrückzahlung zum Zweck des Budgetausgleich in den Kalkülen der Individuen vernachlässigt werden könnten. In diese Richtung gehen die Versuche, die in der Literatur zur Rettung der Groves-Mechanismen unternommen wurden.

So zeigen beispielsweise Green und Laffont (1979) in einem Anwendungsbeispiel für die Clarke-Steuer, daß bei einer sehr hohen Anzahl Beteiligter unter relativ schwachen Anforderungen an die Wahrscheinlichkeitsverteilungen der privaten Informationsparameter das erwartete Aufkommen pro Steuerzahler gegen Null konvergiert. Dementsprechend könne in solchen Fällen, so wird argumentiert, der Effekt einer gleichmäßig proportionalen Wiederausschüttung in den Optimierungsentscheidungen der Individuen von vornherein vernachlässigt werden, weil diese praktisch nicht damit rechnen würden, eine Rückerstattung zu erhalten. ${ }^{16}$

Dieses "bounded rationality argument"17 ist aber aus folgendem Grund nicht sehr überzeugend: Zwar ist in Gegenwart sehr vieler Beteiligter der erwartete Rück-

\footnotetext{
${ }^{16}$ Vgl. Green und Laffont (1979, S. 166 ff.). Ein ähnliches Argument findet sich auch in Tideman und Tullock (1976, S. 1155f.).

${ }^{17}$ Siehe Green und Laffont (1979, S. 165).
} 
transfer pro Kopf sehr klein, dafür ist aber auch der Anreiz eines einzelnen Individuums, seine Präferenzen wahrheitsgemäß zu offenbaren, nur schwach ausgeprägt. Die Nutzeneinbußen, die es der Allgemeinheit durch seine Angaben auferlegt, sind nämlich in großen Gruppen verschwindend gering. Dementsprechend ist auch die erwartete Clarke-Steuer, die es zu zahlen hat, minimal, so daß es im Prinzip vorstellbar ist, daß der Effekt des Rücktransfers den Besteuerungseffekt überwiegt und das Individuum falsche Präferenzen angibt. In diesem Fall können u.U. sehr hohe Wohlfahrtsverluste auftreten, da es keine Garantie dafür gibt, daß die Angaben nicht erheblich von der Wahrheit abweichen. Aus diesem Grund untersuchen Green und Laffont (1979) noch eine andere Modellvariante, die sich stärker an einer allgemeinen Gleichgewichtsbetrachtung orientiert, indem sie mögliche Rücktransfers von vornherein in die individuellen Kalküle miteinbezieht.

In diesem Modell wird angenommen, da $\beta$ die Individuen wissen, da $\beta$ eine Clarke-Steuer zur Anwendung kommen und das Steueraufkommen gleichmäßig an alle Beteiligten zurücktransferiert werden soll. Da der Clarke-Mechanismus unter dieser Voraussetzung die Eigenschaft der dominanten Strategien verliert, muß eine bestimmte Erwartungsbildung der Individuen über die Präferenzangaben ihrer Opponenten modelliert werden. Green und Laffont zeigen nun, daß unter nicht allzu restriktiven Annahmen bezüglich der Wahrscheinlichkeitsverteilungen, die diese Erwartungen beschreiben sollen, die korrekte Präferenzoffenbarung "asymptotisch beste Antwort" bleibt, d.h. die angegebenen Informationen konvergieren gegen die wahren, wenn die Anzahl der Beteiligten gegen Unendlich geht. Da sich aber bei sehr vielen Beteiligten durch die Akkumulation kleiner Abweichungen von der Wahrheit große Wohlfahrtsverluste ergeben können, wird zusätzlich noch nachgewiesen, daß diese gegen Null gehen und der Mechanismus somit auch "approximativ effizient" ist. ${ }^{18}$

Als dritte Möglichkeit, das Problem des Budgetausgleichs in den Griff zu bekommen, schlagen Green und Laffont vor, den Clarke-Mechanismus nur auf einen Teil der betroffenen Individuen anzuwenden und die Allokationsentscheidung auf der Basis dieser Stichprobe zu treffen. Das Aufkommen der Clarke-Steuer könnte dann an den unbefragten Teil ausgeschüttet werden. ${ }^{19}$ Dieses Verfahren, das natürlich auch nur in Situationen mit vielen Beteiligten sinnvoll eingesetzt werden kann, wenn die Stichproben einigermaßen zuverlässig sein sollen, hat zwei offensichtliche

$18 \mathrm{Vgl}$. dazu Green und Laffont (1979, S. $175 \mathrm{ff}$.).

${ }^{19} \mathrm{Vgl}$. Green und Laffont (1979, S. 165 u. S. 213 ff.). 
Schwachpunkte: Erstens führt es zu mehr oder weniger hohen Effizienzverlusten, weil die First-Best-Allokation nur durch eine Totalerhebung ermittelt werden könnte, und zweitens treten wieder die in Teilabschnitt 5.1.2.2 beschriebenen Umverteilungseffekte zwischen dem befragten und dem unbefragten Teil der Betroffenen auf.

Insgesamt kommen Green und Laffont, was die praktische Anwendung von Groves-Mechanismen betrifft, zu dem Schluß, "that the problem of balancing the budget is not a very serious problem for the mechanisms we are studying."20

Diese Aussage mag für Probleme mit sehr vielen Beteiligten zutreffend sein, wenngleich in solchen Situationen gerade unter praktischen Gesichtspunkten noch andere Argumente eine Rolle spielen, die diese Euphorie doch etwas dämpfen dürften. ${ }^{21}$ Wenn man diesen Standpunkt zunächst dennoch akzeptiert, so bestätigen die oben genannten Ergebnisse einmal mehr die Vermutung, daß eine zentrale Internalisierung externer Effekte im Fall großer Gruppen dem Property-Rights-Ansatz überlegen ist: Während die im dritten Kapitel durchgeführten Untersuchungen gezeigt haben, daß in großen Ökonomien eine Verschärfung der Informationsprobleme zum völligen Scheitern einer Verhandlungslösung beiträgt, scheint die Anwendung von Groves-Mechanismen gerade in diesen Fällen die Lösung aller Probleme zu sein. Diese Erkenntnis ist aber im Hinblick auf die zentrale Fragestellung dieses zweiten Teils der Arbeit nur von untergeordneter Bedeutung, da hier der Vergleich zwischen zentralen Lösungsansätzen und einer freiwilligen Internalisierung in Situationen mit wenigen Beteiligten, in denen letztere noch die vergleichsweise größten Erfolge verspricht, im Mittelpunkt steht. In diesen Fällen bestehen aber, wie die Ausführungen dieses Abschnitts deutlich gemacht haben, begründete Zweifel an der Überlegenheit einer zentralen Internalisierung mit Hilfe von Groves-Mechanismen, weil das Problem des Budgetausgleichs überhaupt nicht, bzw. nur unter Inkaufnahme inakzeptabler Verteilungseffekte gelöst werden kann.

\subsection{Ausgeglichenes Budget statt dominanter Strategien: Der Mechanismus von d'Aspremont und Gerard-Varet}

Die vorangegangene Diskussion legt es nahe, nach alternativen Präferenzenthüllungsmechanismen zu suchen, bei denen der Haushalt des zentralen Koordinators

${ }^{20} \mathrm{Vgl}$. Green und Laffont (1979, S. 188).

${ }^{2} \mathrm{Vgl}$. dazu die Diskussion in Abschnitt 5.3. 
ausgeglichen ist. Dabei ist klar, daß diese Eigenschaft nicht "kostenlos" zu haben ist. Da Groves-Mechanismen die einzigen sind, bei denen die Angabe der wahren Präferenzen dominante Strategie ist und das Problem des unausgeglichenen Budgets bei ihrer Anwendung i.d.R. unvermeidlich ist, kann ein Mechanismus mit ausgeglichenem Budget nur durch den Verzicht auf die Eigenschaft der dominanten Strategien erkauft werden.

Wenn die Bekundung ihrer tatsächlichen Präferenzen für die Individuen nicht mehr die bedingungslos beste Antwort darstellt, dann muß der Mechanismus zumindest so konzipiert sein, daß jeder Spieler die Wahrheit sagt, wenn seine Gegenspieler ebenfalls die Wahrheit sagen. Dann ist die Offenbarung der Präferenzen wenigstens ein Gleichgewicht. Diese Anforderung ist natürlich erheblich schwächer. Die Folgen dieser Abschwächung werden in Teilabschnitt 5.2.2.1 noch zu diskutieren sein.

Die Notwendigkeit des Verzichts auf dominante Strategien wirft nun die Frage nach einem geeigneten Gleichgewichtskonzept für das Spiel zwischen den beteiligten Individuen auf. Die Wahl dieses Gleichgewichtskonzepts hängt v.a. davon ab, ob die Agenten über die privaten Informationen ihrer Gegenspieler Bescheid wissen oder nicht. Im ersten Fall handelt es sich um eine Spielsituation mit vollständiger Information. Das adäquate Gleichgewichtskonzept für diese Situation wäre das gewöhnliche Nash-Gleichgewicht. ${ }^{22}$ Dieses Szenario ist aber hier ohne Bedeutung, da in dieser Arbeit die Annahme unvollständiger Information zwischen den Spielern entscheidend ist. Es wäre inkonsistent, bei der privaten Verhandlungslösung zu unterstellen, der Schädiger könne die Schadensfunktion des Geschädigten nicht beobachten, und dann bei der Anwendung eines zentralen Allokationsmechanismus vom Gegenteil auszugehen.

Die Tatsache, da $B$ bei der Implementation in Nash-Gleichgewichten die Durchsetzung effizienter Allokationen mit einem ausgeglichenen Budget vereinbar ist, ist zwar, losgelöst vom Thema dieser Arbeit, sehr interessant. So haben beispielsweise Groves und Ledyard (1977b) einen Mechanismus entwickelt, der auf Nash-Strategien basiert und mit dessen Hilfe effiziente Allokationen mit öffentlichen Gütern bei gleichzeitigem Budgetausgleich implementiert werden können. ${ }^{23}$

${ }^{22}$ Zur Diskussion der Bedingungen, unter denen das Konzept des Nash-Gleichgewichts trotz unvollständiger Information angewendet werden kann, vgl. Trockel (1991).

${ }^{23}$ Groves und Ledyard (1977b) präsentieren ihren Mechanismus im Rahmen eines komplizierten allgemeinen Gleichgewichtsmodells. In Groves und Ledyard (1977a) findet man eine vereinfachte Version in einem Partialmodell. Eine eingängige und auch kritische Darstellung bietet Feldman 
Im Zusammenhang mit dem Öffentlichen-Guts-Problem ist die Konzeption eines solchen Mechanismus auch sinnvoll, da hier mit dem "Trittbrettfahrerproblem" schon unter der Bedingung vollständiger Information eine Ursache für Ineffizienzen vorliegt. In unserem Externalitätenmodell wäre ein solcher Mechanismus aber überflüssig, da bei vollständiger Information schon die Verhandlungslösung effizient wäre. Es wird also im folgenden wieder davon ausgegangen, daß Schädiger und Geschädigter nur ihre eigene private Information kennen und über die Charakteristika ihres Opponenten, ebenso wie die Zentralinstanz, nur wissen, wie sie statistisch verteilt sind. In dieser Situation ist, wie schon in Kapitel 3, das Konzept des Bayesianischen Gleichgewichts anzuwenden.

Gesucht ist jetzt also ein Präferenzenthüllungsmechanismus, bei dem

a) die wahrheitsgemäße Offenbarung der privaten Informationen durch Schädiger und Geschädigten ein Bayesianisches Gleichgewicht darstellt und

b) der Haushalt der Zentralbehörde ausgeglichen ist.

Ein bekannter Mechanismus, der diese Eigenschaften aufweist, wurde, wieder im Zusammenhang mit öffentlichen Gütern, von d'Aspremont und Gerard-Varet (1979) bzw., unabhängig davon, von Arrow (1979) entwickelt. Dieser Mechanismus, für den sich in der Literatur nach den Namen der beiden Erstgenannten die Kurzbezeichnung "AGV-Mechanismus" eingebürgert hat, soll im folgenden auf unser Modell übertragen werden.

\subsubsection{Charakterisierung des AGV-Mechanismus}

Zur Charakterisierung des AGV-Mechanismus wird wieder die Version des einfachen Externalitätenmodells herangezogen, die auch in Abschnitt 5.1 bei der Beschreibung der Groves-Mechanismen verwendet wurde. Es wird also wieder angenommen, die Grenzgewinnfunktion des Schädigers sei durch $b^{\prime}(x, a)$, die Grenzschadensfunktion des Geschädigten durch $d^{\prime}(x, \theta)$ gegeben. Die Funktionen sollen wieder die üblichen Eigenschaften aufweisen. Die Parameter a und $\theta$ sind der

(1982, S. 129ff.). Weniger transparent ist dagegen die Präsentation von Green und Laffont (1979, S. $149 \mathrm{ff}$.). 
regulierenden Behörde unbekannt, der Schädiger kennt nur sein a und der Geschädigte nur $\operatorname{sein} \theta$. Die allgemeine Gestalt der Grenzgewinn- und Grenzschadensfunktion sowie die Wahrscheinlichkeitsverteilungen von a und $\theta$ sind dagegen "common knowledge".

Der AGV-Mechanismus funktioniert nun folgendermaßen: Genau wie bei den Groves-Mechanismen kündigt die Zentralinstanz an, sie werde das erlaubte Produktionsniveau entsprechend Gleichung (5.1) so festlegen, daß der angegebene Grenzgewinn mit dem angegebenen Grenzschaden übereinstimmt. Der Unterschied besteht in der Ausgestaltung der Transferfunktionen, durch die die Enthüllungsanreize gesetzt werden sollen. Die Transferzahlung, die der Geschädigte erhält, wenn er den Informationsparameter $\hat{\theta}$ signalisiert, während der Schädiger $\hat{a}$ angibt, ist jetzt durch

$$
\tau_{\mathrm{G}}=\mathrm{E}_{\mathrm{a}}\left\{\mathrm{b}\left(\mathrm{x}^{*}(\mathrm{a}, \hat{\theta}), \mathrm{a}\right)\right\}+\mathrm{T}_{\mathrm{G}}(\hat{\mathrm{a}})
$$

gegeben. Dabei symbolisiert $\mathrm{E}_{\mathrm{a}}$, daß über den Term in den geschweiften Klammern der (durch $\hat{\theta}$ bedingte) Erwartungswert bezüglich a gebildet wird. Der Transfer, der an den Schädiger gezahlt wird, beträgt

$$
\tau_{\mathrm{s}}=-\mathrm{E}_{\theta}\left\{\mathrm{d}\left(\mathrm{x}^{*}(\hat{\mathrm{a}}, \theta), \theta\right)\right\}+\mathrm{T}_{\mathrm{s}}(\hat{\theta})
$$

wenn er selbst $\hat{a}$ und der Geschädigte $\hat{\theta}$ nennt. Die ersten Terme in diesen beiden Gleichungen sind wieder die Anreizkomponenten, die Funktionen $\mathrm{T}_{\mathrm{G}}(\hat{a})$ und $\mathrm{T}_{\mathrm{S}}(\hat{\theta})$ sind aus der Sicht des jeweiligen Transferempfängers Konstanten und können so gewählt werden, daß das Budget der Zentralinstanz ausgeglichen ist.

Beim AGV-Mechanismus entsprechen also die Anreizkomponenten nicht mehr wie bei den Groves-Mechanismen dem angeblichen Gewinn des Schädigers bzw. dem angeblichen Schaden des Geschädigten bei Implementation von $\mathrm{x}^{*}($.$) .$ Stattdessen wird über diese Ausdrücke der bedingte Erwartungswert bezüglich des Informationsparameters des Opponenten gebildet, so daß sie aus der Sicht des jeweiligen Individuums deterministische Größen darstellen, die nur noch von ihrem eigenen Signal abhängen. Diese Eigenschaft ist ausschlaggebend dafür, daß hier ein Budgetausgleich immer möglich ist, ohne die Anreize zu beeinträchtigen. 
Die Bedingung für einen ausgeglichenen Haushalt lautet jetzt

$$
\mathrm{D}=\tau_{\mathrm{G}+} \tau_{\mathrm{s}}=\mathrm{E}_{\mathrm{a}}\left\{\mathrm{b}\left(\mathrm{x}^{*}(\mathrm{a}, \hat{\theta}), \mathrm{a}\right)\right\}-\mathrm{E}_{\theta}\left\{\mathrm{d}\left(\mathrm{x}^{*}(\hat{\mathrm{a}}, \theta), \theta\right)\right\}+\mathrm{T}_{\mathrm{G}}(\hat{\mathrm{a}})+\mathrm{T}_{\mathrm{s}}(\hat{\theta})=0
$$

Definiert man $\varepsilon_{\mathrm{G}}(\hat{\theta}):=\mathrm{E}_{\mathrm{a}}\left\{\mathrm{b}\left(\mathrm{x}^{*}(\mathrm{a}, \hat{\theta}), \mathrm{a}\right)\right\}$ und $\varepsilon_{\mathrm{S}}(\hat{\mathrm{a}}):=\mathrm{E}_{\theta}\left\{\mathrm{d}\left(\mathrm{x}^{*}(\hat{\mathrm{a}}, \theta), \theta\right)\right\}$, dann läßt sich die Budgetgleichung auch in der Form

$$
\varepsilon_{G}(\hat{\theta})+T_{S}(\hat{\theta})=\varepsilon_{S}(\hat{a})-T_{G}(\hat{a})
$$

schreiben. Man sieht sofort, daß diese Gleichung für alle â und $\hat{\theta}$ identisch erfüllt ist, falls $T_{S}(\hat{\theta})=-\varepsilon_{G}(\hat{\theta})$ und $T_{G}(\hat{a})=\varepsilon_{S}(\hat{a})$ gesetzt wird. Das bedeutet zum einen, da $\beta$ die Anreizsteuer $\varepsilon_{\mathrm{S}}(\hat{a})$, die der Schädiger zu zahlen hat, an den Geschädigten transferiert wird. Dies ist ohne Probleme möglich, da der Transfer aus der Sicht des Geschädigten Lump-Sum-Charakter hat. Darüber hinaus muß der Schädiger aber auch noch den Anreiztransfer $\varepsilon_{\mathrm{G}}(\hat{\theta})$, der an den Geschädigten gezahlt wird, finanzieren. Die Steuer, die dazu erhoben werden muß, ist ebenfalls anreizneutral, da sie aus der Sicht des Schädigers eine Lump-Sum-Steuer ist.

Nachdem klar ist, daß bei dieser Gestaltung der Transferfunktionen ein Budgetausgleich immer möglich ist, muß noch gezeigt werden, daß die wahrheitsgemäße Offenbarung der privaten Informationsparameter ein Bayesianisches Gleichgewicht darstellt. Es muß also nachgewiesen werden, daß es für jedes der beiden Individuen optimal ist, seinen tatsächlichen Informationsparameter anzugeben, wenn dies auch das andere tut.

Betrachten wir dazu zuerst das Kalkül des Geschädigten. Unter der Bedingung, $\mathrm{da} ß$ der Schädiger seinen wahren Informationsparameter a signalisiert, kann sich der Geschädigte mit Hilfe der allgemein bekannten Wahrscheinlichkeitsverteilung von a den Erwartungswert des implementierten Produktionsniveaus und damit seinen erwarteten Schaden ausrechnen, wenn er selbst $\hat{\theta}$ angibt. Dieser ist durch $\mathrm{E}_{\mathrm{a}}\left\{\mathrm{d}\left(\mathrm{x}^{*}(\mathrm{a}, \hat{\theta}), \theta\right)\right\}$ bestimmt, falls $\theta$ sein wahrer Informationsparameter ist. Außerdem ist er in der Lage, den Erwartungswert des (entscheidungsirrelevanten) LumpSum-Transfers $T_{G}(a)=\varepsilon_{S}(a)$ zu berechnen, so daß unter Berücksichtigung der insgesamt in Aussicht gestellten Transferzahlungen sein Erwartungsnutzen

(5.19) $\operatorname{Eu}(\hat{\theta}, \theta)=\tau_{\mathrm{G}}(\hat{\theta})-\mathrm{E}_{\mathrm{a}}\left\{\mathrm{d}\left(\mathrm{x}^{*}(\mathrm{a}, \hat{\theta}), \theta\right)\right\}=$ 


$$
=\mathrm{E}_{\mathrm{a}}\left\{\mathrm{b}\left(\mathrm{x}^{*}(\mathrm{a}, \hat{\theta}), \mathrm{a}\right)-\mathrm{d}\left(\mathrm{x}^{*}(\mathrm{a}, \hat{\theta}), \theta\right)\right\}+\mathrm{E}_{\mathrm{a}}\left\{\varepsilon_{\mathrm{s}}(\mathrm{a})\right\}
$$

beträgt. Aus dieser Gleichung ist unmittelbar ersichtlich, daß die optimale Strategie des Geschädigten darin besteht, $\hat{\theta}=\theta$ zu signalisieren, da der Ausdruck innerhalb der ersten geschweiften Klammer in diesem Fall für alle a maximiert wird, wie in Abschnitt 5.1.1 begründet wurde. Es ist klar, daß unter diesen Umständen auch der Erwartungswert über a maximal ist.

Damit ist erwiesen, daß für den Geschädigten die Angabe des wahren Informationsparameters beste Antwort ist, wenn der Schädiger ebenfalls die Wahrheit sagt. Dies muß nun umgekehrt auch in Bezug auf den Schädiger gezeigt werden. Da die Argumentation analog zur obigen ist, soll es genügen, den erwarteten Gewinn des Schädigers einfach hinzuschreiben. Man erhält

$$
\begin{aligned}
& E \pi(\hat{a}, a)=E_{\theta}\left\{b\left(x^{*}(\hat{a}, \theta), a\right)\right\}+\tau_{s}(\hat{a})= \\
& =E_{\theta}\left\{b\left(x^{*}(\hat{a}, \theta), a\right)-d\left(x^{*}(\hat{a}, \theta), \theta\right)\right\}-E_{\theta}\left\{\varepsilon_{G}(\theta)\right\}
\end{aligned}
$$

Aus dem gleichen Grund wie oben folgt aus dieser Gleichung, daß es auch für den Schädiger optimal ist, die Wahrheit $\hat{a}=$ a zu bekennen, falls dies auch der Geschädigte tut. Damit ist nachgewiesen, da $\beta$ die Angabe der wahren Informationsparameter im Rahmen des AGV-Mechanismus wechselseitig beste Antwort ist und ein Bayesianisches Gleichgewicht darstellt.

\subsubsection{Schwächen des AGV-Mechanismus}

Der vorangegangene Abschnitt hat gezeigt, daß mit dem AGV-Mechanismus ein Instrument zur Verfügung steht, mit dessen Hilfe sowohl das Problem der Informationsenthüllung als auch das Problem des Budgetausgleichs gelöst werden kann. Der Zentralinstanz wäre es also im Prinzip möglich, das effiziente Externalitätsniveau zu implementieren und gleichzeitig sicherzustellen, daß die Internalisierungsgewinne in ihrer Summe vollständig den beteiligten Individuen selbst zugute kommen. In diesem Sinne könnte also der AGV-Mechanismus, im Gegensatz zu den Groves-Mechanismen, als wirklich effizient bezeichnet werden. Trotz dieses positiven Resultats sollten die Erfolgsaussichten des AGV-Mechanismus nicht allzu 
optimistisch beurteilt werden, weil er dafür andere Schwächen aufweist, die fast ebenso gravierend sind, wie die Unmöglichkeit des Budgetausgleichs. Diese Schwächen sollen im folgenden diskutiert werden.

\subsubsection{Schwächen des Gleichgewichtskonzepts}

Wie bereits festgestellt, mußte die Eigenschaft eines ausgeglichenen Budgets um den Preis des Verzichts auf das Gleichgewichtskonzept der dominanten Strategien erkauft werden. Die Tatsache, daß dem AGV-Mechanismus lediglich das Konzept des Bayesianischen Gleichgewichts zugrunde liegt, reduziert die Chancen der Zentralinstanz, das von ihr angestrebte effiziente Enthüllungsgleichgewicht wirklich durchsetzen zu können, in mehrfacher Hinsicht.

So stellt die Implementation in Bayesianischen Gleichgewichten im Vergleich zur Implementation in dominanten Strategien deutlich höhere Anforderungen an den Informationsstand der Zentralinstanz. Das Hauptproblem besteht darin, daß der Koordinator eines Bayesianischen Mechanismus die A-Priori-Vermutungen der Agenten über die private Information ihrer Gegenspieler kennen muß. Dieses Problem wurde in den hier betrachteten Modellversionen verdrängt, da aus Vereinfachungsgründen durchwegs angenommen wurde, es existieren objektive und allgemein bekannte Wahrscheinlichkeitsverteilungen der Informationsparameter. Würde man es sich nicht so einfach machen, müßte man davon ausgehen, daß Schädiger und Geschädigter nur subjektive Vermutungen über die statistische Verteilung der Informationsparameter ihres Rivalen haben. In diesem Fall berechnen die beiden den Erwartungswert des Gewinns bzw. des Nutzens, den sie erreichen können, falls ihr Gegenspieler seine private Information wahrheitsgemä $\beta$ preisgibt, auf der Basis dieser vermuteten Wahrscheinlichkeitsverteilungen. Dementsprechend müßte auch die Zentralinstanz bei der Berechnung der Anreizkomponenten in den Gleichungen (5.15) bzw. (5.16) diese Vermutungen zugrunde legen. Sind ihr diese nicht bekannt, ist sie nicht in der Lage, die richtigen Anreize zu setzen, so daß die Individuen möglicherweise falsche Angaben machen werden. Dabei muß sie damit rechnen, daß diese Angaben u.U. erheblich von der Wahrheit abweichen, selbst wenn die Vermutungen nur geringfügig falsch eingeschätzt wurden. Angesichts dieser Möglichkeit ist die Anwendung des AGV-Mechanismus nur dann sinnvoll, wenn die Zentralinstanz über die Vermutungen der Agenten genau Bescheid weiß. Da es aber 
extrem unrealistisch erscheint, einen derartig hohen Informationsstand vorauszusetzen, ist es höchst fraglich, ob das Enthüllungsgleichgewicht tatsächlich implementiert werden kann. ${ }^{24}$

Abgesehen von dem soeben beschriebenen Informationsproblem sind noch zwei weitere Gründe zu nennen, warum die Aussichten, das Enthüllungsgleichgewicht tatsächlich durchzusetzen, recht gering sein dürften:

Erstens ist in Bayesianischen Spielen die Entscheidungssituation, in der sich die Spieler befinden, ungleich komplexer als in Spielen, in denen sie über dominante Strategien verfuigen. Sie müssen nun auch im Stande sein, das Verhalten ihrer Gegenspieler korrekt zu antizipieren. Die Anforderungen an ihre Fähigkeit, strategisch zu denken, steigen also deutlich. Dies kann zum einen dazu führen, daß die Spieler sich selbst irrational verhalten, zum anderen müssen sie verstärkt mit einer gewissen Irrationalität ihrer Rivalen rechnen und deren eventuelles Fehlverhalten in ihr Kalkül mit einbeziehen. Beides kann dazu führen, daß der AGV-Mechanismus Ergebnisse hervorbringt, die von der Zentralinstanz weder beabsichtigt noch vorhersehbar sind.

Zweitens besteht in Bayesianischen Spielen, wie wir bereits in Teilabschnitt 3.3.3 gesehen haben, die Gefahr des Auftretens multipler Gleichgewichte. Inwieweit auch der AGV-Mechanismus von diesem Phänomen betroffen ist, soll hier nicht weiter untersucht werden. Sollte dies der Fall sein, dann stehen die Spieler insofern vor einem Koordinationsproblem, als sie sich gemeinsam auf eines dieser Gleichgewichte einigen und die entsprechenden Strategien ergreifen müßten. Abgesehen davon, daß unklar ist, ob die Individuen prinzipiell in der Lage sind, Koordinationsprobleme dieser Art zu lösen, ist zumindest fraglich, ob sie ausgerechnet das Enthüllungsgleichgewicht spielen werden. Dies wäre noch am ehesten zu erwarten, wenn sich kein Spieler in einem anderen Gleichgewicht besser stellen könnte, eine Einigung auf das Enthüllungsgleichgewicht also im Interesse aller Beteiligten läge. Dieser Fall erscheint aber angesichts der enormen Umverteilungseffekte zu Lasten des Schädigers eher unwahrscheinlich.

${ }^{24}$ Vgl. zu dieser Argumentation auch Mookherjee und Reichelstein (1992, S. 379). 


\subsubsection{Verteilungsprobleme: Fehlende individuelle Rationalität}

Die bisher angeführten Nachteile des AGV-Mechanismus bezogen sich auf die Schwächen des zugrundeliegenden spieltheoretischen Gleichgewichtskonzepts und das daraus abgeleitete Risiko, das angestrebte effiziente Enthüllungsgleichgewicht am Ende doch nicht zu erreichen. Abgesehen davon kann man dem Mechanismus aber noch einen weiteren Einwand entgegenbringen, der sich auf seine extremen Verteilungswirkungen bezieht.

Wie in Abschnitt 5.2.1 gezeigt wurde, zieht der AGV-Mechanismus eine sehr einseitige Belastung des Schädigers nach sich, da dieser neben seiner Anreizsteuer auch noch den Anreiztransfer für den Geschädigten finanzieren muß, um den Budgetausgleich zu ermöglichen. Dies führt dazu, daß der Mechanismus für den Schädiger nicht mehr "individuell rational" ist, wenn sein Gewinn schon ohne diese Belastung recht niedrig ist. Wie es dazu kommen kann, soll im folgenden gezeigt werden.

Das Konzept der individuellen Rationalität spielte bisher nur im Zusammenhang mit privaten Verhandlungen eine Rolle, wurde aber, was zentral organisierte Allokationsmechanismen betrifft, vernachlässigt. Im Rahmen privater Verhandlungen war individuelle Rationalität eine der Grundvoraussetzungen, die ein Internalisierungsvertrag erfüllen mußte, damit der Geschädigte als akzeptierende Partei bereit war, ihm zuzustimmen. Diese Voraussetzung war erfullt, wenn sich der Geschädigte durch seine Zustimmung im Vergleich zur Ausgangssituation nicht schlechter stellen konnte. Allgemein kann ein Allokationsmechanismus als individuell rational bezeichnet werden, wenn er die Eigenschaft hat, "that all players are ex ante at least as well off from playing the game as from refraining from play."25 Wenn man diese Definition auf den hier behandelten Kontext anwendet, dann bedeutet dies, daß Schädiger und Geschädigter freiwillig, d.h. ohne Zwang durch die Zentralinstanz, die den Allokationsmechanismus koordiniert, zur Teilnahme bereit sind, bevor sie die konkrete Ausprägung des Informationsparameters ihres Opponenten erfahren haben. Die Teilnahmebereitschaft der Individuen hängt davon ab, mit welchen Konsequenzen sie im Falle einer Weigerung rechnen müssen.

Falls die Zentralinstanz bereit ist, auf Zwangsmaßnahmen zu verzichten und den Agenten ein gewisses Maß an "Fairness" entgegenzubringen, dann liegt es nahe, jeden, der sich weigert, seine Präferenzen in den Entscheidungsprozeß einzubringen,

${ }^{25}$ Siehe Laffont und Maskin (1979, S. 299). 
einfach zu ignorieren. Die anstehende Entscheidung wird dann nur auf der Basis der Präferenzangaben derer getroffen, die sich nicht weigern. Unter diesen Umständen würde man bei einer Teilnahmeverweigerung durch den Geschädigten dem Schädiger die uneingeschränkte Ausübung seiner Aktivität gestatten, während der Schädiger mit einem vollständigen Produktionsverbot rechnen muß, falls er sich weigert. Der Reservationsgewinn des Schädigers wäre in diesem Fall Null, der Reservationsnutzen des Geschädigten entspräche dem Erwartungswert des Schadens, den er erleiden würde, wenn der Schädiger sein individuelles Gewinnmaximum realisieren würde.

Der AGV-Mechanismus ist bei Zugrundelegung dieser Außenoptionen nur dann individuell rational, wenn der erwartete Gewinn bzw. der erwartete Nutzen, den die beiden Individuen im Enthüllungsgleichgewicht erreichen, mindestens so hoch ist wie die jeweilige Reservationsauszahlung. Für den Schädiger muß also unabhängig von seinem tatsächlichen Informationsparameter a

$$
E \pi(a)=E_{\theta}\left\{b\left(x^{*}(a, \theta), a\right)-d\left(x^{*}(a, \theta), \theta\right)\right\}-E_{\theta}\left\{\varepsilon_{G}(\theta)\right\} \geq 0
$$

und für den Geschädigten unabhängig von seinem tatsächlichen Informationsparameter $\theta$

$$
\mathrm{Eu}(\theta)=\mathrm{E}_{\mathrm{a}}\left\{\mathrm{b}\left(\mathrm{x}^{*}(\mathrm{a}, \theta), \mathrm{a}\right)-\mathrm{d}\left(\mathrm{x}^{*}(\mathrm{a}, \theta), \theta\right)\right\}+\mathrm{E}_{\mathrm{a}}\left\{\varepsilon_{\mathrm{s}}(\mathrm{a})\right\} \geq \mathrm{E}_{\mathrm{a}}\{\mathrm{d}(\overline{\mathrm{x}}(\mathrm{a}), \theta)\}
$$

gelten. Dabei bezeichnet $\bar{x}(a)$ die individuell optimale Produktionsmenge des Geschädigten, falls er vom Typ a ist.

$\mathrm{Da} B$ diese Bedingungen i.a. verletzt sind, wird anhand eines einfachen Beispiels, das in Anhang $\mathrm{C}$ nachzulesen ist, demonstriert. In diesem Beispiel zeigt sich, daß der AGV-Mechanismus für den Schädiger nicht individuell rational ist, wenn seine Gewinnsituation von vornherein schon "zu ungünstig" ist. Eine solche Situation liegt in fast $90 \%$ aller Fälle vor, d.h. der Schädiger würde mit sehr hoher Wahrscheinlichkeit nicht am Mechanismus teilnehmen, wenn ihm diese Option offenstünde.

Die Frage, ob ein zentralisierter Allokationsmechanismus überhaupt individuell rational sein muß, ist in der Literatur recht umstritten. Einige Autoren sind der Ansicht, daß sich die Zentralinstanz nicht an diese Beschränkung zu halten braucht. So schreibt z.B. Illing (1992): "Of course, in the presence of private information, a 
central agency must take into account the incentive compatibility constraints in the same way as private firms have to do. But the conditions of individual rationality are no longer binding."26 Bei Farrell (1987) liest man: "Central authority (...) can also help efficiency by making recalcitrant people participate in schemes that benefit society in general."27

$\mathrm{Da} B$ die Zentralinstanz "widerspenstige Leute" zur Teilnahme an einem Mechanismus zwingen kann, dürfte kaum zu bestreiten sein. Dazu bräuchte sie, beispielsweise durch Androhung einer entsprechend hohen Strafe, nur dafür zu sorgen, $\mathrm{da} B$ der Reservationsnutzen eines Individuums im Falle einer Weigerung hinreichend niedrig ist. Dies ist aber nicht das eigentliche Problem. Die entscheidende Frage ist, ob solche Zwangsmaßnahmen angewandt werden sollen. Da diese Frage auf die Verteilungswirkungen des AGV-Mechanismus abzielt, kann sie nur vor dem Hintergrund subjektiver Wertvorstellungen beantwortet werden. Würde man der Auffassung von Illing oder Farrell folgen, so würde man sich damit zufriedengeben, $\mathrm{da} \beta$ der Mechanismus die gesamtwirtschaftliche Wohlfahrt, als Nutzensumme betrachtet, maximiert, auch wenn manche Individuen dabei verlieren. Eine solche Position wird üblicherweise mit dem Argument untermauert, daß man sich Kompensationsmaßnahmen überlegen könnte, um die Verlierer zu entschädigen. Was nun den AGV-Mechanismus betrifft, so ist eine derartige Kompensation nicht möglich, da die Transfer- und Steuerzahlungen durch die Bedingungen der Anreizverträglichkeit und des Budgetausgleichs zwingend festgelegt sind. Damit bringt er, etwa in den 90\% aller Fälle des Beispiels aus Anhang C, in denen er nicht individuell rational ist, im Vergleich zum Status Quo keine Wohlfahrtssteigerung im Sinne des ParetoKriteriums. ${ }^{28}$ So betrachtet erscheint die Ansicht von Illing und Farrell etwas problematisch, da der Effizienzgedanke unter Mißachtung des Pareto-Kriteriums m.E. stark an Zugkraft verliert. Wenn man aus ethisch-moralischen Gründen jedem Individuum ex ante die Chance erhalten will, wenigstens seinen Reservationsnutzen zu erreichen, muß man sich nach einem alternativen Mechanismus umsehen, der die Bedingung der individuellen Rationalität nicht verletzt und gleichzeitig die Enthüllung der privaten Informationen sowie den Budgetausgleich sicherstellt.

${ }^{26}$ Vgl. Illing (1992, S. 569).

27Vgl. Farrell (1987, S. 121).

28 Man beachte, daß die Bedingung der individuellen Rationalität ohnehin nur eine durchschnitliche Pareto-Verbesserung verlangt. Es wird nicht gefordert, daß für jede denkbare Realisation der privaten Informationen ein pareto-überlegener Zustand realisiert wird. 
Die Hoffnung, einen solchen Mechanismus zu finden, wird durch ein weiteres "Unmöglichkeitstheorem" zerschlagen, das auf eine Arbeit von Laffont und Maskin (1979) zurückgeht. In dieser Arbeit wird in einem Modellrahmen, der in seiner Grundstruktur starke Parallelen zum hier verwendeten Modell aufweist, zunächst allgemein die Klasse der Mechanismen charakterisiert, die zur wahrheitsgemäßen Enthüllung der individuellen Präferenzen in einem Bayesianischen Gleichgewicht führen und ein ausgeglichenes Budget gewährleisten. Dabei wird gezeigt, daß diese Anforderungen i.a. nur erfüllbar sind, wenn es sich bei der sozialen Wohlfahrtsfunktion, die durch die Anwendung eines der betreffenden Mechanismen maximiert werden soll, um eine gewichtete Summe individueller Nutzen handelt. Es stellt sich heraus, daß der AGV-Mechanismus als Spezialfall resultiert, wenn die individuellen Nutzen gleich gewichtet werden. ${ }^{29}$ Im nächsten Schritt wird dann nachgewiesen, daß ein Mechanismus mit den genannten Eigenschaften i.d.R. nicht individuell rational sein kann. ${ }^{30}$

Dieses Resultat wird auch durch Myerson und Satterthwaite (1983) bestätigt. In dieser Arbeit wird ein Verhandlungsproblem zwischen dem potentiellen Käufer und dem potentiellen Verkäufer eines unteilbaren Gutes betrachtet, das über einen neutralen Vermittler gelöst werden soll. Die Wertschätzungen der beiden Tauschpartner sind dabei private Information. Aufgabe des Vermittlers ist es, einen Tauschmechanismus zu konzipieren, der effizient sein soll, indem er das Gut demjenigen Individuum zuteilt, dessen wahre Wertschätzung höher ist. Dieser Tauschmechanismus muß natürlich auch individuell rational sein. Es wird gezeigt, daß ein Mechanismus, der alle diese Anforderungen gleichzeitig erfüllt, i.a. nicht existiert, wenn keine Subventionen von außen zufließen, d.h. wenn das Budget des Vermittlers nicht ausgeglichen ist. Obgleich dieses Modell aufgrund seiner Struktur, anders als das von Laffont und Maskin, nicht unmittelbar auf die hier behandelte Problematik übertragen werden kann, geht das zentrale Ergebnis doch in die gleiche Richtung. ${ }^{31}$

${ }^{29}$ Eine solche Wohlfahrtsfunktion wurde in dieser Arbeit implizit meist unterstellt. Vergleiche aber z.B. die Überlegungen zur individuellen Rationalität.

${ }^{30}$ Es wird gezeigt, daß die beschriebenen Mechanismen allenfalls dann als individuell rational bezeichnet werden können, wenn sie zu einem Zeitpunkt angewendet werden, zu dem die Individuen ihre eigenen Informationsparameter noch nicht kennen.

${ }^{31}$ In den Kapiteln 2 und 3 der vorliegenden Arbeit wurde gezeigt, daß Verhandlungslösungen bei unvollständiger Information ineffizient sind, wenn man von vollständiger Dezentralisierung ausgeht, d.h. wenn man annimmt, daß einer der Verhandlungspartner die Verhandlungsprozedur bestimmen kann. Durch die Arbeit von Myerson und Satterthwaite (1983) wird dieses Ergebnis noch 
Wie man die in diesem Abschnitt herausgearbeiteten Schwächen des AGVMechanismus im Hinblick auf einen Vergleich mit der privaten Verhandlungslösung wertet, hängt zunächst davon ab, ob man trotz aller möglichen Bedenken gegenüber dem zugrundeliegenden Gleichgewichtskonzept daran glaubt, daß das Enthüllungsgleichgewicht tatsächlich zustande kommt. Tut man dies nicht, wird man wohl eher geneigt sein, auf die Verhandlungslösung zu bauen. Sofern man dem Schädiger zutraut, eine so komplexe Kontraktgestaltung, wie sie im zweiten Kapitel beschrieben wurde, auszuarbeiten, ist die Verhandlungsprozedur selbst recht einfach: Die offerierende Partei macht den ersten Spielzug und die akzeptierende Partei überlegt sich daraufhin ihre beste Antwort. Diese ist im bilateralen Modell in jedem Fall eindeutig. Der AGV-Mechanismus ist, was die Anforderungen an den "Designer" betrifft, sicher nicht weniger komplex. Bei ihm handelt es sich aber um ein Simultanspiel, d.h. die Agenten müssen ihre Strategien bestimmen, ohne den Spielzug ihres Gegenspielers beobachten zu können. Dieser Umstand macht den Spielausgang v.a. in Abwesenheit dominanter Strategien viel unsicherer, weil er zum einen höhere Ansprüche an die strategischen Fähigkeiten der Spieler stellt und zum anderen typischerweise zu multiplen Gleichgewichten führt.

Von dieser Warte aus betrachtet wirkt die Verhandlungslösung in gewisser Weise zuverlässiger und es ist zu überlegen, ob man ihr trotz ihrer unvollkommenen Effizienzeigenschaften nicht den Vorzug gegenüber einem Allokationsmechanismus geben sollte, dessen Ergebnis zwar effizient ist, aber bei realistischer Betrachtung kaum zustande kommen wird.

Wenn man, was das Zustandekommen des Enthüllungsgleichgewichts betrifft, weniger pessimistisch ist, dann muß man sich fragen, ob eine mögliche Verletzung der Bedingung individueller Rationalität in Kauf genommen werden soll. Wer der Ansicht ist, daß Wohlfahrtsverbesserungen für die Allgemeinheit nicht durch Nutzeneinbußen einzelner Individuen erkauft werden dürfen, wird eine Ressourcenverteilung, wie sie der AGV-Mechanismus hervorbringen kann, ablehnen und eher die Verhandlungslösung präferieren. Diese muß ja unter allen Umständen individuell rational sein, weil man niemanden zur Teilnahme an Verhandlungen zwingen kann. Das Kriterium der Freiwilligkeit, das dem Property-Rights-Ansatz zugrunde liegt, schützt also die Individuen vor "staatlicher Willkür" und stellt sicher, daß beim

verstärkt: Nicht einmal dann, wenn eine wohlwollende Zentralinstanz den Verhandlungsprozeß bestimmen könnte, dürfte man sich effiziente und individuell rationale Verhandlungslösungen erwarten. Daraus folgt, daß Verhandlungen bei unvollständiger Information generell ineffizient sind. 
Übergang vom Status Quo zum Zustand nach Abschluß der Verhandlungen eine Pareto-Verbesserung stattfindet.

Bei einer abschließenden Beurteilung der Präferenzenthüllungsmechanismen wird man zugeben müssen, daß sie selbst dann, wenn man ihren Einsatz in der Praxis prinzipiell für möglich hält, nicht als Allheilmittel gegen das Informationsproblem angesehen werden können. Das Auftreten unvollständiger Information bringt offensichtlich immer in der einen oder anderen Form Wohlfahrtsverluste mit sich: Im Rahmen einer privaten Internalisierung offenbaren sich diese Verluste in Form von nicht ausgeschöpften Verhandlungspotentialen. Bei der Anwendung von Groves-Mechanismen treten entweder Budgetdefizite auf, die durch externe Mittel finanziert werden müssen, oder es kommt zu Haushaltsüberschüssen, die nicht an die betroffenen Individuen ausgeschüttet werden können. Will man den Budgetausgleich sichern, so muß man auf individuelle Rationalität verzichten, d.h. manchen Individuen das Recht absprechen, "Nein" zu einem bestimmten Allokationsverfahren zu sagen. Auch der Verlust dieses Rechts kann, freilich nicht im Sinne der klassischen "welfare economics", als eine Art "Wohlfahrtsverlust" interpretiert werden.

\subsection{Praktische Einwände gegen Präferenzenthüllungsmechanismen}

In diesem Kapitel wurde bisher überwiegend aus einem rein theoretischen Blickwinkel argumentiert, indem davon ausgegangen wurde, die beschriebenen Enthüllungsmechanismen seien im Hinblick auf reale Anwendungen prinzipiell praktikabel. $\mathrm{Da} B$ diese Annahme allenfalls als Arbeitshypothese dienen kann, wird offensichtlich, wenn man sich die Schwierigkeiten vergegenwärtigt, die sich bei einem tatsächlichen Einsatz in der Praxis ergeben würden. In diesem Abschnitt soll auf einige dieser Schwierigkeiten eingegangen werden.

Tatsache ist, daß Präferenzenthüllungsmechanismen, wie sie hier diskutiert wurden, in der realen Welt offensichtlich keinerlei Verwendung finden. Dabei liegt der Verdacht nahe, daß dafür am wenigsten ihre theoretischen Schwächen verantwortlich sind.

Ein möglicher Grund für diese Bedeutungslosigkeit könnte darin zu suchen sein, daß die Anwendung dieser Mechanismen ein politisches System voraussetzt, in dem die Individuen ihre Präferenzen unmittelbar in den gesellschaftlichen Entschei- 
dungsprozeß einbringen können. ${ }^{32}$ Wie Johansen (1977) im Zusammenhang mit dem Free-Rider-Problem bei der Bereitstellung öffentlicher Güter betont hat, werden aber die meisten Entscheidungen im politischen Leben nicht unmittelbar von den betroffenen Individuen selbst, sondern von ihren gewählten Vertretern getroffen. In einer repräsentativen Demokratie komme es daher im Stadium der Entscheidungsfindung nicht mehr auf die Präferenzangaben der Individuen, sondern auf die der Politiker an. Das Problem der Falschangabe von Präferenzen spiele unter diesen Umständen gar keine Rolle, da die Repräsentanten in der politischen Diskussion, in der sie versuchen, einander von ihren eigenen Positionen zu überzeugen, ihre wahren Präferenzen zumindest teilweise enthüllen würden. Die Politiker könnten gar kein Interesse daran haben, in einer solchen Diskussion die Präferenzen ihrer Wähler zu verschleiern, da sie damit rechnen müßten, daß ein solches Verhalten zu Entscheidungen führen könnte, die letztendlich von niemandem gewollt (z.B. eine dauerhafte Unterversorgung mit öffentlichen Gütern) und den Wählern gegenüber schwer zu vertreten seien. Selbst wenn sich ein einzelner Politiker relativ sicher sein könnte, daß seine Gegner keine Free-Rider-Positionen einnehmen würden und eine Falschangabe generell zum Vorteil seiner Wähler wäre, könnte er es sich dennoch kaum leisten, sich beispielsweise im Wahlkampf im Widerspruch zu deren Präferenzen zu äußern, da er mit Stimmenverlusten rechnen müßte, wenn die Wähler seine Strategie nicht durchschauen. Aus diesen Gründen sei das Problem der Präferenzverschleierung allenfalls eine Randerscheinung. Nicht zuletzt deshalb bezeichnet Johansen (1981) in einer Besprechung des im Rahmen dieser Arbeit mehrmals zitierten Buches von Green und Laffont (1979) die Bemühungen um die Entwicklung von Präferenzenthüllungsmechanismen als "rather academic exercises". 33

Diese Überlegungen sind, wenngleich theoretisch kaum ausgearbeitet, sicherlich bedenkenswert, was Entscheidungen über öffentliche Güter anbelangt, von denen eine Vielzahl von Individuen betroffen sind. Hier steht aber die Frage im Mittelpunkt, inwieweit Präferenzenthüllungsmechanismen bei Problemen mit einer geringen Anzahl von Beteiligten als Alternative zu privaten Verhandlungen, die ebenfalls gerade in solchen Situationen halbwegs erfolgversprechend sind, eingesetzt werden können. In diesen Fällen greift Johansens Argumentation nicht mehr, weil die Entscheidungsfindung in kleinen Gruppen doch eher auf direkt-demokratischem Wege abläuft. Man denke in etwa an das klassische bilaterale Externalitätenproblem zwi-

32 Vgl. Bohm (1979, S. 143.)

${ }^{33} \mathrm{Vgl}$. Johansen (1981, S. 128). 
schen einer Papierfabrik, die Schadstoffe in einen Fluß einleitet, und einem Fischereibetrieb, der diesen Fluß als Fanggrund nutzt. Es wäre ziemlich abwegig, anzunehmen, ein solches Problem werde über gewählte Repräsentanten gelöst. Man wird vielmehr erwarten, daß sich die beiden Firmen entweder in freiwilligen Verhandlungen gütlich auf eine akzeptable Lösung einigen, oder daß eine lokale Behörde eine verbindliche Regelung trifft. In letzterem Fall müßte sich die Behörde die für eine möglichst effiziente Regulierung nötigen Informationen unmittelbar bei den Betroffenen beschaffen, so daß das Problem der Präferenzverschleierung durchaus auftreten kann und der Einsatz eines Enthüllungsmechanismus grundsätzlich in Erwägung zu ziehen wäre. Auch Johansen räumt ein, daß die Falschangabe von Präferenzen in kleinen Gruppen ein Problem sein kann. ${ }^{34}$ Die Frage ist dann, warum Enthüllungsmechanismen selbst in solchen Situationen nicht angewendet werden.

Die üblicherweise angeführten administrativen Schwierigkeiten dürften dafür nur teilweise verantwortlich sein. Sicherlich müßte eine Reihe von Problemen der Datenerhebung und -verarbeitung gelöst werden. So wäre in jedem Fall eine direkte Befragung der Individuen, etwa durch den Einsatz geeigneter Fragebögen und Interviewer erforderlich, was u. U. recht kostspielig sein kann, v.a. wenn das Externalitätsproblem zwar nur wenige Individuen betrifft, aber dennoch weit verbreitet ist. Einen weiteren Kostenfaktor könnte die Notwendigkeit der Entwicklung spezieller Computerprogramme darstellen, die in der Lage sein müßten, aus den erhobenen Daten das optimale Niveau der Entscheidungsvariablen und die Anreizsteuern bzw. -transfers zu berechnen. Diese Hürden treten aber zum einen hauptsächlich in Situationen mit vielen Beteiligten auf, die hier weniger zur Diskussion stehen, und dürften zum anderen in Anbetracht der anhaltenden Fortschritte auf den Gebieten der Kommunikationstechnik und der elektronischen Datenverarbeitung noch am leichtesten zu überwinden sein.

Erheblich größere Schwierigkeiten dürfte schon die qualitative Natur der zu erhebenden Daten bereiten. Um das effiziente Externalitätsniveau implementieren zu können, muß die Zentralinstanz den Grenznutzen und die Grenzkosten der betreffenden Aktivität in Erfahrung bringen. Dies ist noch vergleichsweise unproblematisch, wenn es sich dabei um monetäre Größen handelt, wie etwa die Grenzgewinnfunktion des Schädigers in unserem einfachen Externalitätenmodell. Häufig geht es aber gerade im Umweltbereich um monetär schwer erfaßbare oder gar immaterielle Kosten und Nutzen, wie z.B. körperliche oder psychische Schäden. Die

${ }^{34} \mathrm{Vgl}$. Johansen (1977, S. 147 f.). 
Frage ist dann, wie solche Größen gemessen und bewertet werden sollen, oder noch viel grundsätzlicher, wonach man die Individuen in diesen Fällen konkret befragen soll. 35

Das entscheidende Hindernis, das einer Anwendung von Präferenzenthüllungsmechanismen auf reale Probleme im Wege steht, scheint aber m.E. in ihrer Komplexität zu liegen. Ein Mechanismus, der praktikabel sein soll, muß in erster Linie für alle Beteiligten, also sowohl für die Agenten, als auch für den zentralen Koordinator, leicht verständlich sein. $\mathrm{Daß}$ die hier beschriebenen Mechanismen dieses Kriterium nicht erfüllen, dürfte von kaum jemandem ernsthaft bestritten werden. Ihre Funktionsweise dürfte zumindest für einen ökonomischen Laien so schwer zu durchschauen sein, da $\beta$ man selbst nach eindringlicher Erläuterung nicht erwarten kann, die Agenten davon überzeugen zu können, es sei für sie am besten, ihre privaten Informationen wahrheitsgemäß zu offenbaren. Dies wird insbesondere dann der Fall sein, wenn die Individuen, wie beim AGV-Mechanismus, nicht über dominante Strategien verfügen. ${ }^{36} \mathrm{Ng}$ (1979) äußert seine Skepsis gegenüber derartig hohen Ansprüchen an den Durchschnittsbürger auf folgende Weise: "...anyone who has experience in marking undergraduate examination papers will have doubts that an average citizen is able to achieve such an understanding." ${ }^{37}$

Dieses Argument gilt nicht nur für die am Mechanismus Beteiligten, sondern auch für die Behörden, die ihn anwenden und koordinieren sollen. Es kann ja nicht unbedingt vorausgesetzt werden, daß diese den nötigen Sachverstand besitzen, insbesondere dann nicht, wenn es sich um lokale Behörden, wie z.B. um eine Stadtoder Kreisverwaltung handelt. Farrell (1987) schreibt in diesem Zusammenhang: "Property rights and voluntary private negotiation fail to achieve 'first-best' efficient outcomes when there is important private information. And such outcomes often can be achieved, despite the information problems, by a wise and benevolent king who is prepared to coerce people to participate in an incentive scheme. But there has been no such king since Solomon. Central authority may be better portrayed by the image of a bureaucrat, perhaps benevolent but certainly bumbling."38

\footnotetext{
${ }^{35}$ Eine ausführliche Diskussion von Problemen und Methoden der empirischen Erfassung von Präfe-renzen für öffentliche Güter bietet Pommerehne (1987).

${ }^{36}$ Beim AGV-Mechanismus dürften selbst ausgebildete Ökonomen ihre Bedenken haben, auch wenn sie seine Funktionsweise verstehen.

${ }^{37} \mathrm{Vgl}$. Ng (1979, S. 205).

38Vgl. Farrell (1987, S. 122).
} 
Die Komplexität der Präferenzenthüllungsmechanismen erstreckt sich, abgesehen von den Schwierigkeiten, die bei ihrer Durchführung aufgrund von Unzulänglichkeiten auf Seiten aller beteiligten Entscheidungsträger zu erwarten sind, auch auf die Möglichkeit ihrer politischen Durchsetzung. Bohm (1979) bemerkt dazu, daß die Einführung solcher Mechanismen als neue Instrumente der Entscheidungsfindung sehr unwahrscheinlich sei, weil zum einen die verantwortlichen Politiker, selbst oft ökonomische Laien, nur schwer von den Vorzügen dieser Allokationsverfahren zu überzeugen seien. Zum anderen könnten diese Mechanismen aus dem selben Grund auch von den Wählern nicht akzeptiert werden, so daß der Druck aus der breiten Bevölkerung, der nötig wäre, um die Politiker zur Einführung solcher Instrumente auch gegen ihren Willen zu zwingen, fehle. ${ }^{39}$

Alle diese Argumente geben begründeten Anlaß zu der Vermutung, daß die in diesem Kapitel diskutierten Allokationsmechanismen auch in Zukunft keine Chancen auf einen tatsächlichen Einsatz haben werden, sondern "akademische Übungen" bleiben werden. Die Tatsache, daß Präferenzenthüllungsverfahren hier trotz dieser Erkenntnis relativ ausführlich behandelt wurden, erscheint dadurch gerechtfertigt, daß sie, wie die unüberschaubare Fülle an einschlägigen Literaturbeiträgen zeigt, mit einem erheblichen Aufwand an wirtschaftstheoretischer Grundlagenforschung als "ideale" Lösungen von Informationsproblemen bei zentralisierten Entscheidungsprozessen entwickelt wurden. Aus diesem Grund darf eine, wenn auch längst nicht erschöpfende, Auseinandersetzung mit diesen Ansätzen in einer Arbeit, die sich mit der Internalisierung externer Effekte bei unvollständiger Information beschäftigt, nicht fehlen. Dies gilt umso mehr, als sich herausgestellt hat, daß diese scheinbar so perfekten Verfahren gerade auch aus theoretischer Sicht verschiedene Schwächen aufweisen. Einige dieser Schwächen überblicksartig und ohne die in der Literatur übliche formale Überfrachtung darzustellen war das Ziel der Ausführungen dieses Kapitels. Es dürfte deutlich geworden sein, daß die Präferenzenthüllungsmechanismen weit davon entfernt sind, Informations- und Allokationsprobleme in befriedigender Weise zu lösen.

${ }^{39} \mathrm{Vgl.} \mathrm{Bohm} \mathrm{(1979,} \mathrm{S.} 144$ f.). 


\section{Einfache staatliche Internalisierungsinstrumente im Vergleich zur Verhandlungslösung}

Im vorangegangenen Kapitel wurden einige theoretische und praktische Gründe angegeben, die Präferenzenthüllungsmechanismen als Instrumente zur Implementation effizienter Allokationsergebnisse ungeeignet erscheinen lassen. In diesem Kapitel wird nun der Frage nachgegangen, inwieweit eine relativ anspruchslose, dafür aber praktikable Internalisierungspolitik, die von ihrer Konzeption her von vornherein auf die Enthüllung privater Informationen verzichtet, eine wirkungsvolle Alternative zu privaten Verhandlungen sein kann.

\subsection{Eine Praktikable Alternative: Durchschnittlich optimale Regulierung}

Eine brauchbare Politik zur Internalisierung externer Effekte sollte einerseits frei sein von den theoretischen Mängeln, die sich für die Enthüllungsmechanismen als verhängnisvoll erwiesen haben. Dazu gehört v.a. die Unmöglichkeit eines Budgetausgleichs, die das Hauptproblem bei den Mechanismen der Groves-Klasse darstellt, sowie die Verletzung der Bedingung individueller Rationalität, die speziell beim AGV-Mechanismus festgestellt wurde, aber auch bei anderen Mechanismen auftreten kann. Andererseits sollte diese Alternative nicht zu komplex, sondern sowohl für die privaten Wirtschaftseinheiten als auch für die regulierende Zentralinstanz leicht verständlich und handhabbar sein.

Diese Kriterien werden sicherlich von einer Pigou-Steuer oder einer Auflage erfüllt, die nicht auf jede mögliche Konstellation entscheidungsrelevanter privater Informationen optimal zugeschnitten ist, sondern davon unabhängig so festgesetzt wird, daß die gesamtwirtschaftliche Wohlfahrt wenigstens im statistischen Durchschnitt über die Informationsparameter maximal ist. Diese Maßnahmen sind praktikabel, da sie der Zentralinstanz ein relativ geringes $\mathrm{Ma}$ an Wissen über die Präferenzen bzw. Technologien der privaten Wirtschaftseinheiten abverlangen und aufgrund ihrer Einfachheit und Klarheit nicht mißverstanden werden können. Insbesondere sind keine strategischen Überlegungen mehr notwendig, da die Individuen vor der Bestimmung des Steuersatzes bzw. der Auflage nicht nach ihren Präferenzen befragt werden. 
Darüber hinaus ist es beim Einsatz dieser Instrumente immer möglich, einen Budgetausgleich herbeizuführen. Bei einer Auflagenlösung fallen ohnehin keine Steuern oder Transferzahlungen an. Bei einer Abgabenlösung kann das erzielte Aufkommen an den Geschädigten transferiert werden, ohne daß dadurch irgendwelche Verzerrungen ausgelöst würden. ${ }^{1}$ Eine solche Regelung hätte den Vorteil, daß der Geschädigte im Durchschnitt für die erlittenen Schäden kompensiert werden könnte und wäre deshalb auch unter Verteilungsgesichtspunkten akzeptabel. Auch die Bedingung der individuellen Rationalität wird nicht verletzt: Der Geschädigte würde sowohl bei einer Steuer- als auch bei einer Auflagenlösung im Vergleich zur Laisser-Faire-Situation, in der es dem Schädiger erlaubt wäre, sein persönliches Gewinnmaximum zu realisieren, auf keinen Fall schlechter gestellt. Was den Schädiger betrifft, so würde dieser im ungünstigsten Fall seine Produktion einstellen müssen, wenn der Pigou-Steuersatz überall höher ist als sein Grenzgewinn, oder wenn ihm ein vollständiges Produktionsverbot auferlegt würde.

Der Nachteil solcher durchschnittlich optimaler Politiken besteht logischerweise darin, daß das Allokationsergebnis, das sie hervorbringen, ex post nur zufällig effizient ist. Dies gilt aber, wenngleich aus anderen Gründen, auch für die private Verhandlungslösung, die hier als Vergleichsmaßstab dienen soll. Die Idee, einen solchen "Second-Best-Vergleich" zwischen privater Verhandlungslösung und einfachen zentralen Internalisierungsmaßnahmen durchzuführen, geht auf die Arbeit von Farrell (1987) zurück. Er legt seiner Gegenüberstellung ein Modell zugrunde, das in seiner Struktur unserem bilateralen Externalitätenmodell sehr ähnlich ist. Der Verhandlungsproze $B$, den er verwendet, basiert ebenfalls auf der Fähigkeit der offerierenden Partei, "Take-it-or-leave-it"-Offerten zu unterbreiten. Die Verhandlungslösung bei unvollständiger Information wird mit einer durchschnittlich optimalen Auflagenlösung verglichen. Farrell geht bei der Berechnung der optimalen Auflage davon aus, da $B$ der Zentralinstanz die Grenznutzenfunktionen beider Individuen unbekannt sind. Es zeigt sich, daß bei der Auflagenlösung je nach Konstellation der

\footnotetext{
${ }^{1}$ Dies gilt allerdings nur im Rahmen unseres einfachen Externalitätenmodells, in dem der Geschädigte keine Möglichkeit hat, selbst aktive Maßnahmen zur Schadensbegrenzung zu ergreifen. Wäre dies anders, so würde eine Kompensation des Geschädigten dazu führen, daß sein eigener Aufwand zur Schadensbegrenzung ineffizient niedrig ist. (Vgl. dazu Baumol und Oates (1988, S. 45 ff.)). Ein Rücktransfer an den Schädiger würde dagegen in jedem Fall verzerrend wirken: Wenn der Schädiger eine Rückzahlung antizipiert, sind seine erwarteten Zusatzkosten pro Produktionseinheit niedriger als der Pigou-Steuersatz, und er wird entsprechend mehr produzieren.
} 
Parameter seines Modells sowohl höhere als auch niedrigere durchschnittliche Wohlfahrtsgewinne möglich sind.

Buchholz und Haslbeck (1991/1992) sowie Illing (1992) haben Farrells Argument aufgegriffen und in einem einfacheren Modellrahmen transparenter gemacht. Beide Arbeiten verwenden ein Modell, das sich von Farrells und dem hier verwendeten Modell im wesentlichen darin unterscheidet, daß die privaten Informationsparameter nur zwei unterschiedliche Ausprägungen annehmen können. In der ersten Arbeit wird die Verhandlungslösung mit einer Auflagenlösung, in der zweiten Arbeit mit einer Abgabenlösung verglichen. Der Vergleich erfolgt jeweils unter der Annahme einer allgemein bekannten Gewinnfunktion des Schädigers, während die Schadensfunktion des Geschädigten unbekannt ist. Auch hier stellt sich heraus, daß die durchschnittlich optimale Politik durchaus effizienter sein kann als die Verhandlungslösung.

Ziel dieses Kapitels ist es, einen solchen Wohlfahrtsvergleich in unserem einfachen Externalitätenmodell durchzuführen, wobei sich die Präsentation, abgesehen von Unterschieden in der Modellspezifikation, v.a. in zwei Punkten von den drei oben zitierten Aufsätzen unterscheidet:

Zum einen wird hier in einem einheitlichen Modellrahmen sowohl das Szenario, in dem die Grenzgewinnfunktion des Schädigers "common knowledge" ist, als auch das Szenario mit allgemein unbekannter Grenzgewinnfunktion untersucht, um herauszufinden, wie sich die zusätzliche Erschwernis, die sich für die Zentralinstanz im zweiten Fall ergibt, auf den Wohlfahrtsvergleich auswirkt.

Zum anderen wird einer Erkenntnis von Weitzmann (1974) Rechnung getragen, derzufolge Preis- und Mengenregulierung bei Unsicherheit über die Grenznutzen und Grenzkosten der zu regulierenden Aktivität i.d.R. nicht mehr äquivalent sind. ${ }^{2}$ Es erscheint also sinnvoll, zunächst zu untersuchen, wann in unserem einfachen Externalitätenmodell eine Auflage, wann eine Pigou-Steuer die durchschnittlich höheren Wohlfahrtsgewinne ermöglicht. Es wird dann davon ausgegangen, da $\beta$ Farrells "bumbling bureaucrat" immerhin in der Lage ist, das effizientere der beiden Instrumente zu erkennen und die bessere Alternative zu ergreifen. In erster Linie ist dann das unter dieser Voraussetzung erzielte Allokationsergebnis mit dem Ergebnis der privaten Verhandlungen zu vergleichen.

${ }^{2}$ Vgl. dazu auch Adar und Griffin (1976), Fishelson (1976) sowie Baumol und Oates (1988, S. 57 ff.). 


\subsection{Second-Best-Vergleich zwischen privaten Verhandlungen und zentraler Regulierung}

Bevor der Second-Best-Vergleich zwischen privater Verhandlungslösung und den oben vorgeschlagenen staatlichen Internalisierungsmaßnahmen durchgeführt wird, sollte darauf hingewiesen werden, daß die abgeleiteten Resultate nur im Rahmen des verwendeten Modells gelten und unter dem Aspekt der Allgemeingültigkeit nicht überbewertet werden dürfen. Es sollen nur beispielhaft gewisse Tendenzen aufgezeigt werden, die sich allerdings auch in den oben zitierten Arbeiten in abgeschwächter Form bestätigen. Mehr kann man auch nicht erwarten, da für einen solchen Vergleich eine Reihe konkreter Berechnungen vorzunehmen ist, deren Resultate zwangsläufig von der Modellspezifikation, insbesondere von der Gestalt der Gewinn- und Schadensfunktion, sowie von Art und Wahrscheinlichkeitsverteilung der unbekannten Informationsparameter abhängen.

Da das Verhandlungsmodell aus Kapitel 2 die Vergleichsgrundlage bilden soll, ist die Modellspezifikation schon weitgehend vorgegeben. Um den formalen Aufwand bei der Ermittlung der Verhandlungslösung in Grenzen zu halten, wurde dort von einer Schadensfunktion mit konstantem marginalen Schaden ausgegangen. Diese Annahme hat zwar, wie wir noch sehen werden, für die Gegenüberstellung von Auflagen- und Abgabenlösung einerseits und den Vergleich mit dem Verhandlungsergebnis andererseits ziemlich gravierende Konsequenzen. Wenn man aber bedenkt, da $B$ es hier nur darum geht, ein konkretes Beispiel durchzurechnen und sich den begrenzten Aussagegehalt dieses Vorhabens bewußt macht, erscheint es gerechtfertigt, an dieser Annahme festzuhalten, zumal eine entsprechende Verallgemeinerung des Verhandlungsmodells mit zusätzlichem Aufwand verbunden wäre, der sich angesichts der im folgenden noch zu treffenden restriktiven Zusatzannahmen kaum lohnen dürfte. Es soll also wieder

(6.1) $d(x, \theta)=\theta x$

gelten.

Was die Gewinnfunktion des Schädigers betrifft, so ist es günstig, den Funktionstyp

$$
b(x, a)=a x-(c / 2) x^{2}
$$


$\mathrm{zu}$ unterstellen, da andernfalls die Berechnungen verschiedener Erwartungswerte, die später durchgeführt werden müssen, mangels Linearität in den stochastischen Informationsparametern stark erschwert würden. ${ }^{3}$

In bezug auf Art und Stochastik der privaten Informationen wird angenommen, $\mathrm{da} B$ a und $\theta$ unbekannt sind, wobei $\theta$ im Intervall $\left[\theta^{-}, \theta^{+}\right]$gleichverteilt sein soll. Die Abgeschlossenheit dieses Intervalls ist zwingend, da der in Kapitel 2 verwendete Formalismus nur unter dieser Voraussetzung angewendet werden kann. ${ }^{4}$ Abgesehen davon ist es aber auch inhaltlich sinnvoll anzunehmen, daß die Schadensfunktion sich nur innerhalb bestimmter Bandbreiten bewegen kann. Da nur ein abgeschlossenes Intervall für $\theta$ zugelassen sein soll, scheiden schon viele der gebräuchlichen Wahrscheinlichkeitsverteilungen, z.B. die Normalverteilung, aus. Die Gleichverteilungsannahme erscheint hier als vernünftige Arbeitshypothese, insbesondere dann, wenn, abgesehen von den Intervallgrenzen, keine weiteren Informationen vorliegen. Die Wahrscheinlichkeitsverteilung von a ist für die folgenden Analysen ohne Belang.

\subsubsection{Preis- vs. Mengenregulierung bei bekannter Gewinnfunktion}

Betrachten wir zuerst den einfacheren Fall, in dem nur die Schadensfunktion des Geschädigten unbekannt ist. Wenn die Zentralinstanz in dieser Situation die erwartete Wohlfahrt

$$
W_{e}=E[b(x, a)-d(x, \theta)]=a x-(c / 2) x^{2}-x E(\theta)
$$

maximieren will, ist es gleichgültig, ob sie dabei auf eine Steuer oder auf eine Auflage zurückgreift. Diese Tatsache kann man sich anhand von Abbildung 18 leicht klarmachen:

\footnotetext{
${ }^{3}$ Dies dürfte auch der Grund dafür sein, daß sich Weitzman (1974) einige Mühe mit der Linearisierung seiner Grenznutzen- und Grenzkostenkurven gemacht hat, und Adar und Griffin (1976) bzw. Fishelson (1976) die Linearität gleich von Beginn an angenommen haben.

${ }^{4} \mathrm{Vgl}$. die Berechnung der Informationsrenten in Teilabschnitt 2.4.1.3.3.
} 


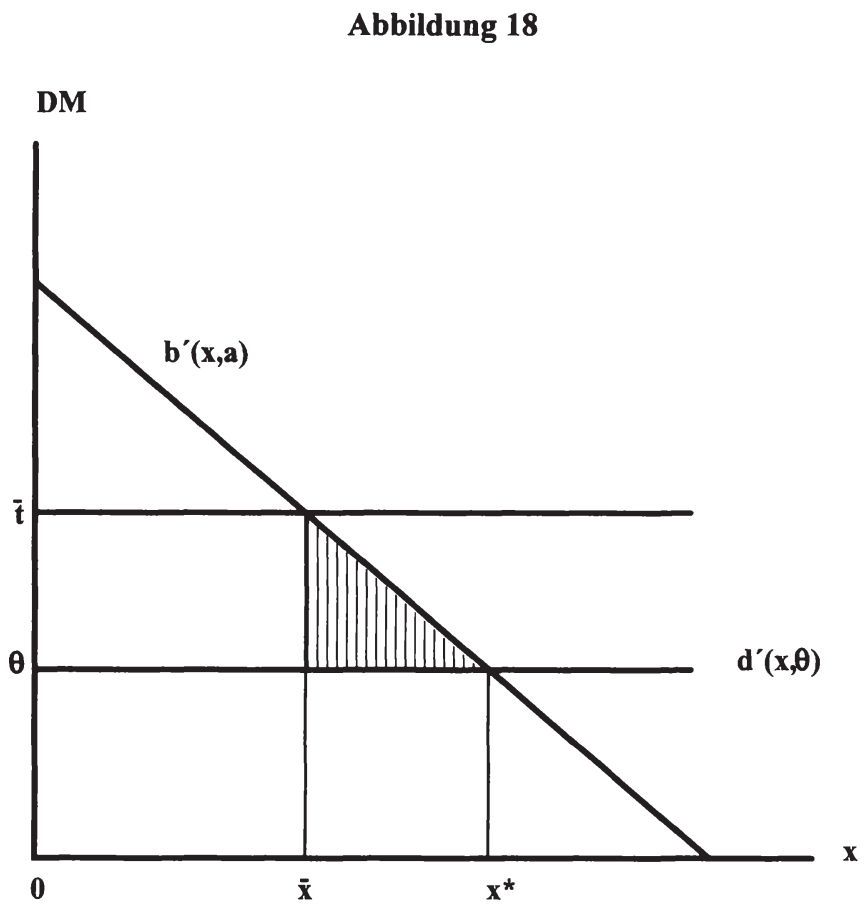

Bei der Auflagenlösung würde die Zentralinstanz dem Schädiger die Überschreitung eines bestimmten Produktionsniveaus $\overline{\mathrm{x}}$ verbieten. Die Einhaltung von $\overline{\mathrm{x}}$ kann sie aber auch auf indirektem Weg durchsetzen, indem sie dem Schädiger pro Outputeinheit eine Steuer in Höhe von $\overline{\mathrm{t}}$ abverlangt. Da die Grenzgewinnfunktion $b^{\prime}(\mathrm{x}, \mathrm{a})$ $=\mathrm{a}-\mathrm{cx}$ als bekannt vorausgesetzt wurde, kann sie sich für jeden beliebigen Steuersatz $\mathrm{t}$ das Produktionsniveau ausrechnen, das der Schädiger gemäß seiner individuellen Gewinnmaximierungsbedingung, $\mathrm{a}-\mathrm{cx}=\mathrm{t}$, wählen wird. Das resultierende Externalitätsniveau ist also nicht nur bei der Auflagenlösung, sondern auch bei der Abgabenlösung eine deterministische Größe. Nur der Wohlfahrtsverlust, der sich ex post einstellt, weil das jeweilige Instrument nicht für jede Situation optimal ist, hängt von der konkreten Ausprägung des Grenzschadens ab und ist damit eine stochastische Größe.

Wie aus Gleichung (6.3) unmittelbar ersichtlich ist, lautet die Bedingung für die durchschnittlich optimale Auflage $\overline{\mathbf{x}}$ 
(6.4) $a-c \bar{x}=E(\theta)$,

so daß sich

(6.5) $\overline{\mathrm{x}}=[\mathrm{a}-\mathrm{E}(\boldsymbol{\theta})] / \mathrm{c}$

ergibt. Der Pigou-Steuersatz, der dazu führt, da $\beta \overline{\mathrm{x}}$ eingehalten wird, ist durch

(6.6) $\overline{\mathrm{t}}=\mathrm{E}(\boldsymbol{\theta})$

gegeben. Den Wohlfahrtsverlust für eine gegebene Realisation von $\theta$ erhält man auf einfache Weise durch Berechnung des Inhalts der schraffierten Dreiecksfläche in Abbildung 18. Es resultiert

$$
\bar{v}(\theta)=(1 / 2)\left[x^{*}(\theta)-\bar{x}\right]\left[b^{\prime}(\bar{x})-\theta\right]=(1 / 2 c)[E(\theta)-\theta]^{2}
$$

wobei neben $(6.5)$ noch $x^{*}(\theta)=(a-\theta) / c$ berücksichtigt wurde. Der erwartete Wohlfahrtsgewinn ist dann durch

$$
\overline{\mathrm{v}}_{\mathrm{c}}=E(\overline{\mathrm{v}}(\theta))=(1 / 2 \mathrm{c}) \int_{\theta^{-}}^{\theta^{+}}[E(\theta)-\theta]^{2} f(\theta) d \theta=(1 / 2 c) \sigma_{\theta}^{2}
$$

bestimmt. Dabei steht $\sigma_{\theta}^{2}$ für die Varianz von $\theta$.

\subsubsection{Preis- vs. Mengenregulierung bei unbekannter Gewinnfunktion}

Wenn man realistischerweise davon ausgeht, daß die Zentralinstanz neben der Schadensfunktion auch die Gewinnfunktion des Schädigers nicht beobachten kann, dann sind Preis- und Mengenregulierung nicht mehr äquivalent. Die erwartete Wohlfahrt, die maximiert werden muß, um die durchschnittlich optimale Auflage zu ermitteln, ist jetzt durch

$$
W_{e}=E[b(x, a)-d(x, \theta)]=E(a) x-(c / 2) x^{2}-x E(\theta)
$$

gegeben. Die zugehörige Optimalitätsbedingung lautet 
(6.10) $\mathrm{E}(\mathrm{a})-\mathrm{c} \overline{\mathrm{x}}=\mathrm{E}(\theta)$,

woraus die Auflage

(6.11) $\overline{\mathrm{x}}=[\mathrm{E}(\mathrm{a})-\mathrm{E}(\theta)] / \mathrm{c}$

folgt.

Soll dagegen eine Pigou-Steuer mit Satz $\mathrm{t}$ erhoben werden, so ist zunächst zu berücksichtigen, daß der Schädiger gemäß seiner individuellen Optimalitätsbedingung $a-c x=t$ den Output $x(a, t)=(a-t) / c$ herstellen wird. Die erwartete Wohlfahrt in Abhängigkeit des zu bestimmenden Steuersatzes beträgt dann

$$
\begin{aligned}
& \mathrm{W}_{\mathrm{e}}=\mathrm{E}[\mathrm{b}(\mathrm{x}(\mathrm{a}, \mathrm{t}), \mathrm{a})-\mathrm{d}(\mathrm{x}(\mathrm{a}, \mathrm{t}), \theta)]= \\
& =(1 / \mathrm{c})\left\{\mathrm{E}\left(\mathrm{a}^{2}\right)-\mathrm{tE}(\mathrm{a})-(1 / 2)\left[\mathrm{E}\left(\mathrm{a}^{2}\right)-2 \mathrm{tE}(\mathrm{a})+\mathrm{t}^{2}\right]-\mathrm{E}(\mathrm{a} \theta)+\mathrm{tE}(\theta)\right\}
\end{aligned}
$$

Als Bedingung erster Ordnung für ein Maximum erhält man, genau wie in der Situation mit bekannter Gewinnfunktion,

(6.13) $\overline{\mathrm{t}}=\mathrm{E}(\theta)$.

Dieses Resultat ist nicht überraschend: Damit die erwartete Wohlfahrt maximal ist, muß der Pigou-Steuersatz dem erwarteten Grenzschaden im Optimum entsprechen. $\mathrm{Da}$ aber der Grenzschaden unabhängig vom Produktionsniveau ist, spielt die Grenzgewinnfunktion für die Bestimmung des Steuersatzes keine Rolle. Deshalb ergibt sich ex post auch das gleiche Externalitätsniveau wie im Szenario mit bekannter Gewinnfunktion. Aufgrund seiner Abhängigkeit vom jetzt unbekannten Informationsparameter a ist es aber jetzt eine stochastische Größe. Um diesen Sachverhalt zum Ausdruck zu bringen, soll das Externalitätsniveau nun zur Abgrenzung gegenüber Gleichung (6.5) durch

(6.14) $\hat{\mathrm{x}}(\mathrm{a})=[\mathrm{a}-\mathrm{E}(\theta)] / \mathrm{c}$

definiert werden. 
Die Nichtäquivalenz von Auflagen- und Abgabenlösung bei unbekannter Grenzgewinnfunktion wird deutlich, wenn man Abbildung 19 betrachtet:

\section{Abbildung 19}

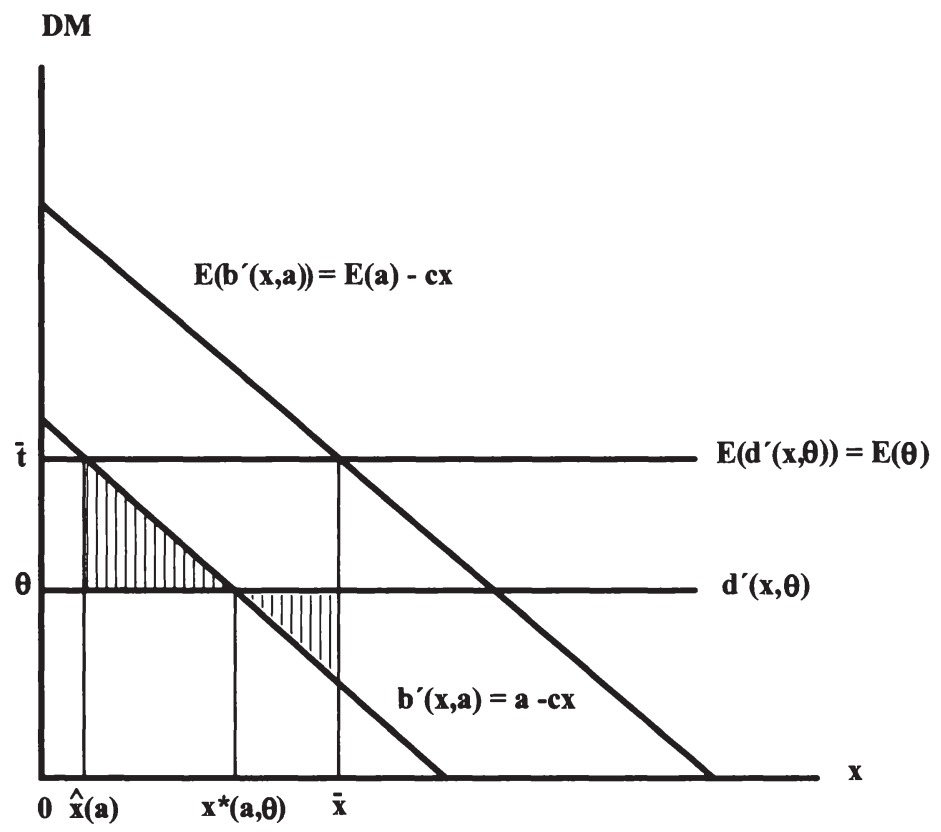

In dieser Abbildung sind die wahre Grenzgewinn- und die wahre Grenzschadensfunktion, sowie deren Erwartungswerte eingezeichnet. Unter den dargestellten Bedingungen wäre $x^{*}(a, \theta)$ das effiziente Externalitätsniveau. Bei der Auflagenlösung dürfte die Menge $\overline{\mathrm{x}}$ nicht überschritten werden, während bei der Steuerlösung mit dem Steuersatz $\overline{\mathrm{t}}=\mathrm{E}(\theta)$ der Schädiger den Output $\hat{\mathrm{x}}(\mathrm{a})$ wählen würde. Die Wohlfahrtsverluste, die sich bei dieser speziellen Realisation der unbekannten Informationsparameter ex post ergeben würden, sind durch die schraffierten Dreiecke dargestellt. Offensichtlich ist der Wohlfahrtsverlust bei der Auflagenlösung kleiner als bei der Abgabenlösung. Diese Relation würde aber zugunsten der Abgabenlösung umschlagen, wenn beispielsweise $\theta$ höher wäre und $x^{*}$ und $\hat{x}$ näher beieinander liegen würden. 
Um entscheiden zu können, welches Instrument das überlegene ist, müssen die erwarteten Wohlfahrtsverluste berechnet werden. Für die Steuerlösung erhält man zunächst ex post mit

$$
\overline{\mathrm{v}}^{\mathrm{s}}(\mathrm{a}, \theta)=(1 / 2)\left[\mathrm{x}^{*}(\mathrm{a}, \theta)-\hat{\mathrm{x}}(\mathrm{a})\right]\left[\mathrm{b}^{\prime}(\hat{\mathrm{x}}(\mathrm{a}), \mathrm{a})-\theta\right]=(1 / 2 \mathrm{c})[\mathrm{E}(\theta)-\theta]^{2}
$$

den gleichen Wohlfahrtsverlust wie im Fall mit bekannter Gewinnfunktion. Da diese Größe wegen des unterstellten Verlaufs von Grenzgewinn- und Grenzschadenskurve unabhängig von a ist, stimmen auch die erwarteten Wohlfahrtsverluste überein. ${ }^{5}$ Es gilt demnach

$$
\overline{\mathrm{v}}_{\mathrm{e}}^{\mathrm{s}}=\mathrm{E}\left(\overline{\mathrm{v}}^{\mathrm{s}}(\mathrm{a}, \theta)\right)=(1 / 2 \mathrm{c}) \sigma_{\theta}^{2}
$$

Es zeigt sich also, daß die Pigou-Steuer unter diesen, zugegebenermaßen sehr speziellen Rahmenbedingungen, die Unsicherheit bezüglich der Gewinnfunktion vollständig neutralisiert.

Die Auflagenlösung erweist sich dagegen als weniger leistungsfähig. Die Wohlfahrtseinbußen, die sich ex post ergeben, sind durch

$$
\bar{v}^{a}(a, \theta)=(1 / 2)\left[x^{*}(a, \theta)-\bar{x}\right]\left[b^{\prime}(\bar{x}, a)-\theta\right]=(1 / 2 c)\{[a-E(a)]-[E(\theta)-\theta]\}^{2}
$$

bestimmt. Der durchschnittliche Wohlfahrtsverlust berechnet sich unter der Voraussetzung, daß a und $\theta$ stochastisch unabhängig sind, zu

$$
\begin{aligned}
& \overline{\mathrm{v}}_{\mathrm{e}}^{\mathrm{a}}=\mathrm{E}\left(\overline{\mathrm{v}}^{\mathrm{a}}(\mathrm{a}, \theta)\right)= \\
& =(1 / 2 \mathrm{c}) \int_{\theta^{-}}^{\theta^{+}} \int_{\mathrm{a}^{-}}^{\mathrm{a}^{+}}\{[\mathrm{a}-\mathrm{E}(\mathrm{a})]-[\theta-E(\theta)]\}^{2} g(a) f(\theta) d a d \theta= \\
& =(1 / 2 c)\left(\sigma_{\mathrm{a}}^{2}+\sigma_{\theta}^{2}\right) .
\end{aligned}
$$

Dabei sind $g(a)$ und $f(\theta)$ die, vorerst beliebigen, Dichtefunktionen der beiden Informationsparameter. Die Auflagenlösung schneidet demnach umso schlechter ab, je

${ }^{5}$ Die Unabhängigkeit des Wohlfahrtsverlustes von a kommt u.a. dadurch zustande, daß die möglichen Grenzgewinnkurven gleiche Steigungen aufweisen. Deshalb fällt a bei der Subtraktion von $\mathrm{x}^{*}$ und $\hat{\mathrm{x}}$ weg. 
größer $\sigma_{\mathrm{a}}^{2}$, die Varianz von a, ist. Dieses Ergebnis ist aus folgendem Grund sehr einleuchtend: Die Wohlfahrtsverluste, die bei der Pigou-Steuer auftreten, sind ausschließlich auf die stochastischen Schwankungen des, annahmegemäß konstanten, Grenzschadens zurückzuführen. Wäre der Grenzschaden bekannt, könnte der externe Effekt auch ohne Informationen über die Grenzgewinnfunktion vollständig internalisiert werden, da der Steuersatz $\mathrm{t}=\boldsymbol{\theta}$ für jede beliebige Realisation von a optimal wäre. Wie weit die Auflage vom Optimum entfernt ist, hängt dagegen auch von der Grenzgewinnfunktion ab. Die erwarteten Wohlfahrtsverluste sind deshalb umso höher, je stärker die Grenzgewinnfunktion schwankt.

\subsubsection{Vergleich mit der Verhandlungslösung}

Nachdem die durchschnittlich zu erwartenden Wohlfahrtsverluste von Auflagen- bzw. Abgabenlösung bei bekannter und unbekannter Gewinnfunktion bestimmt wurden, ist jetzt noch der erwartete Wohlfahrtsverlust, der bei der Verhandlungslösung auftritt, zu berechnen. Wie in Kapitel 2 gezeigt wurde, ergibt sich das Produktionsniveau $x_{v}(\theta)$, das der Schädiger wählt, falls sich der Geschädigte als ein $\theta$-Typ offenbart hat, aus der Bedingung

(6.19) $b^{\prime}(x)=\theta+F(\theta) / f(\theta)$.

Unter unseren speziellen Annahmen über die Grenzgewinnfunktion wird diese Bedingung $z u$

(6.20) $a-c x=\theta+F(\theta) / f(\theta)$,

so daß

$$
x_{v}(\theta)=\{a-[\theta+F(\theta) / f(\theta)]\} / c
$$

das Verhandlungsergebnis ist. Diese Allokation wurde zusammen mit der effizienten Allokation $\mathrm{x}^{*}(\theta)$ in Abbildung 20 dargestellt. Der Wohlfahrtsverlust, den die Verhandlungslösung ex post nach sich zieht, ergibt sich durch Berechnung des Inhalts der schraffierten Dreiecksfläche. 


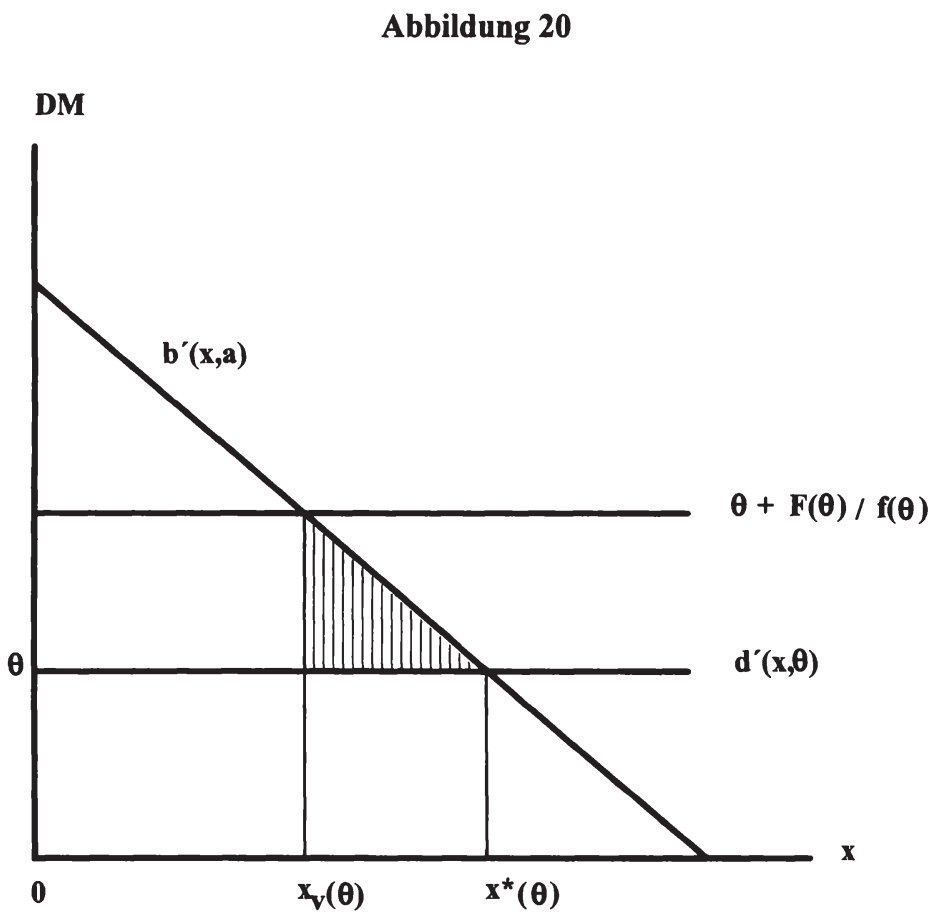

Man erhält

$$
v(\theta)=(1 / 2)\left[x^{*}(\theta)-x_{v}(\theta)\right][F(\theta) / f(\theta)]=(1 / 2 c)[F(\theta) / f(\theta)]^{2}
$$

wobei wieder $x^{*}(\theta)=(a-\theta) / c$ berücksichtigt wurde. Der erwartete Wohlfahrtsverlust beträgt dann

(6.23) $v_{\mathrm{e}}=\mathrm{E}(\mathrm{v}(\theta))=(1 / 2 \mathrm{c}) \mathrm{E}[\mathrm{F}(\theta) / \mathrm{f}(\theta)]^{2}$.

Da in unserem Modell Einkommenseffekte in bezug auf das Externalitätsniveau keine Rolle spielen und folglich, wie Maskin und Tirole (1990) gezeigt haben, das eventuelle Auftreten privater Information beim Schädiger als offerierender Partei für das Verhandlungsergebnis unerheblich ist, gilt Gleichung (6.23) sowohl für das Szenario mit bekannter, als auch für das mit unbekannter Gewinnfunktion. 
Auch im Hinblick auf die Wohlfahrtsverluste, die bei der Internalisierung durch die Zentralinstanz auftreten, braucht nicht zwischen den beiden Fällen unterschieden zu werden, wenn man davon ausgeht, daß die Zentralinstanz ausschließlich daran interessiert ist, ein möglichst hohes durchschnittliches Wohlfahrtsniveau zu erzielen, und sich für die überlegene Abgabenlösung entscheidet. Unter dieser Voraussetzung besteht eine vollkommene Äquivalenz nicht nur zwischen Preis- und Mengenregulierung bei sicherer Gewinnfunktion, sondern auch zwischen Preisregulierung bei sicherer und unsicherer Gewinnfunktion.

Inwieweit der Staatseingriff höhere Wohlfahrtsgewinne erwarten läßt als die private Verhandlungslösung, hängt also bei optimalem Verhalten der Zentralinstanz von der Differenz

$$
\Delta_{e}=v_{e}-\bar{v}_{e}=v_{e}-\bar{v}_{e}^{s}=(1 / 2 c)\left\{E[F(\theta) / f(\theta)]^{2}-\sigma_{\theta}^{2}\right\}
$$

ab. Nur wenn $\Delta_{\mathrm{e}}<0$ ist, ist die Verhandlungslösung die bessere Alternative. Andernfalls erweist sich der Staatseingriff als mindestens gleichwertig, obwohl er auf die Enthüllung der privaten Informationen verzichtet.

Das Ergebnis dieser Gegenüberstellung hängt in entscheidendem Maße von der Wahrscheinlichkeitsverteilung von $\theta \mathrm{ab}$. Diesbezüglich wurde eine Gleichverteilung im Intervall $\left[\theta^{-}, \theta^{+}\right]$angenommen, so $\mathrm{da} B \mathrm{~F}(\theta) / \mathrm{f}(\theta)=\theta-\theta^{-}$gilt. Unter diesen Umständen ergibt sich

$$
\Delta_{e}=(1 / 2 c)\left\{E\left(\theta-\theta^{-}\right)^{2}-\sigma_{\theta}^{2}\right\}=(1 / 2 c)\left\{E\left(\theta-\theta^{-}\right)^{2}-E[\theta-E(\theta)]^{2}\right\}
$$

Dieser Ausdruck ist aber wegen $\mathrm{E}(\theta)>\theta$ eindeutig positiv, weshalb folgende Schlußfolgerungen möglich sind:

Falls die Gewinnfunktion des Schädigers bekannt ist, kann der Staat entweder mit Hilfe einer Pigou-Steuer oder einer äquivalenten Auflage, die lediglich die erwartete Wohlfahrt maximiert, im Durchschnitt ein besseres Allokationsergebnis erreichen als auf dem Verhandlungswege erzielt werden kann. Ist die Gewinnfunktion des Schädigers unbekannt, ändert sich an dieser Überlegenheit (auch quantitativ) nichts, sofern eine Pigou-Steuer erhoben wird.

Dieses Ergebnis ist natürlich extrem, was den letzten Teil der Schlußfolgerung betrifft, der ja nur deshalb zustande kam, weil ein konstanter Grenzschaden angenommen wurde. Dennoch erscheint es legitim, gerade diesen Extremfall zu betrach- 
ten, weil er auf drastische Weise demonstriert, daß eine wohlüberlegte Auswahl eines einfachen und praktikablen Internalisierungsinstruments auf der Basis öffentlich zugänglicher Informationen in bestimmten Situationen eindeutig besser sein kann, als die Verhandlungslösung, bei der unter Aufwendung von Kosten private Informationen erst enthüllt werden müssen.

Wem dieses Beispiel zu suggestiv erscheint, der sei auf die oben zitierten Beiträge verwiesen, die einen ähnlichen Wohlfahrtsvergleich durchführen. Die Resultate, die sich in diesen Arbeiten ergeben haben, sprechen zwar weniger eindeutig gegen die Verhandlungslösung, beweisen aber, daß die Überlegenheit solch einfacher Politiken auch unter anderen Rahmenbedingungen immerhin möglich ist.

Diese Tendenz bestätigt sich, wenn man im Rahmen des hier verwendeten Modells nochmals das Szenario mit unbekannter Gewinnfunktion betrachtet und annimmt, die Zentralinstanz entscheide sich nicht für eine Pigou-Steuer, sondern für die weniger effiziente Auflage. Diese Wahl könnte beispielsweise dadurch begründet werden, daß die Zentralinstanz stark risikoavers ist und Schwankungen des Externalitätsniveaus, wie sie bei der Abgabenlösung auftreten würden, vermeiden möchte. Ein anderer Grund für eine Entscheidung zugunsten der Auflagenlösung könnte darin bestehen, daß eine finanzielle Belastung des Schädigers aus verteilungspolitischen Gründen nicht erwünscht ist oder zu hohe Verwaltungskosten nach sich zieht. In diesem Fall erhält man als Wohlfahrtsdifferenz

$$
\Delta_{\mathrm{e}}^{\prime}=(1 / 2 \mathrm{c})\left\{\mathrm{E}\left(\theta-\theta^{-}\right)^{2}-\sigma_{\theta}^{2}-\sigma_{\mathrm{a}}^{2}\right\}=(1 / 2 \mathrm{c})\left\{\left[\mathrm{E}(\theta)-\theta^{-}\right]^{2}-\sigma_{\mathrm{a}}^{2}\right\}
$$

Berücksichtigt man in (6.26), daß wegen der Gleichverteilungsannahme von $\theta$ im Intervall $\left[\theta^{-}, \theta^{+}\right] \mathrm{E}(\theta)=\left(\theta^{-}+\theta^{+}\right) / 2$ gilt, dann ergibt sich

$$
\Delta_{\mathrm{e}}^{\prime}=(1 / 2 \mathrm{c})\left\{(1 / 2)\left[\theta^{+}-\theta^{-}\right]^{2}-\sigma_{\mathrm{a}}^{2}\right\}=(1 / 2 \mathrm{c})\left\{3 \sigma_{\theta}^{2}-\sigma_{\mathrm{a}}^{2}\right\}
$$

wobei $\sigma_{\theta}^{2}=(1 / 12)\left(\theta^{+}-\theta^{-}\right)^{2}$ verwendet wurde. Dieser Ausdruck ist dann positiv, wenn $\sigma_{\mathrm{a}}^{2} / \sigma_{\theta}^{2}<3$. Das bedeutet immerhin, daß selbst die Auflagenlösung noch besser ist als die private Verhandlungslösung, solange die Varianz von a die von $\theta$ um weniger als den Faktor 3 übersteigt.

Welche Schlußfolgerungen können nun aus den Ergebnissen dieses Wohlfahrtsvergleichs gezogen werden? Es würde sicher zu weit führen, daraus eine unbedingte Überlegenheit staatlicher Internalisierungsmaßnahmen ableiten zu wollen. 
Dazu sind die Modellannahmen viel zu restriktiv und nicht "objektiv" genug. Insbesondere die Annahme eines konstanten Grenzschadens erwies sich als relativ hart, was die Deutlichkeit der Überlegenheit einer zentralen Regulierung betrifft. Dennoch gibt es auch triftige Gründe für die Betrachtung gerade dieses Spezialfalles:

Zum einen konnte deutlich gemacht werden, daß u.U. noch erhebliche zusätzliche Wohlfahrtsgewinne erzielt werden können, wenn der Staat das "richtige" Instrument auswählt. Mit Hilfe einer Pigou-Steuer konnte die Unsicherheit der Gewinnfunktion vollständig neutralisiert und die bei solchen "Daumenregeln" unvermeidlichen Effizienzverluste minimiert werden. Derartige Spielräume bei der Gestaltung staatlicher Maßnahmen dürfen daher bei einem Wohlfahrtsvergleich mit privaten Verhandlungen nicht unberücksichtigt bleiben.

Zum anderen hätte es wenig Sinn gehabt, das Rechenbeispiel so zu konstruieren, daß sich im Ergebnis die Verhandlungslösung als vorteilhafter erwiesen hätte. In diesem Fall wäre nur das alte - und offensichtlich falsche - Vorurteil bestätigt worden, daß bei unvollständiger Information über Technologien und Präferenzen dezentrale Allokationsmechanismen die besseren Resultate liefern. So aber wird man freiwilligen Internalisierungsverhandlungen eher mit einer gewissen Skepsis begegnen. Die Tatsache, da $\beta$ schon derartig simple Maßnahmen ausreichen, um die Effizienz der Verhandlungslösung zu übertreffen, dürfte einem doch zu denken geben. Dies gilt gerade für den Wohlfahrtsvergleich im Szenario mit unbekannter Grenzgewinnfunktion. Wenn diese "common knowledge" ist, ist die Überlegenheit einfacher Internalisierungsinstrumente vielleicht weniger überraschend, da in diesem Fall kein Unterschied zwischen dem Informationsstand der Zentralinstanz und dem des Schädigers besteht. Die informationelle Ausgangssituation ist unter beiden institutionellen Rahmenbedingungen die gleiche. Etwas erstaunlicher ist dagegen das schlechte Abschneiden der Verhandlungslösung bei unbekannter Grenzgewinnfunktion. Dieses gewinnt angesichts der in der Einführung erwähnten Auffassung Hayeks an Bedeutung, derzufolge man die Entscheidungen über die Ressourcenallokation aus Effizienzgründen den privaten Wirtschaftseinheiten überlassen sollte, weil diese die nötigen Informationen über Präferenzen und Technologien besitzen. Vor dem Hintergrund dieser Behauptung würde man vermuten, daß die Verhandlungslösung im Vergleich zum zentralen Internalisierungsansatz gerade dann höhere Wohlfahrtsgewinne erwarten läßt, wenn die Gewinnfunktion des Schädigers nur diesem selbst bekannt ist. Unter diesen Umständen hätte der Schädiger gegenüber der regulierenden Behörde tatsächlich einen Informationsvorsprung, der sich nach 
Hayeks Argumentation in Form einer vergleichsweise höheren Effizienz der Verhandlungslösung niederschlagen müßte. Die Ergebnisse unseres Rechenbeispiels haben gezeigt, daß sich diese Vermutung i.a. nicht bestätigt.

\subsubsection{Synthese zwischen Verhandlungen und Staatseingriffen}

Wie wir gesehen haben, müssen einfache zentrale Internalisierungsmaßnahmen, die auf Informationsenthüllung verzichten, nicht unbedingt die schlechteste Lösung darstellen. Die Wohlfahrtsverluste, die dadurch automatisch hingenommen werden, können durchaus geringer sein als die Ineffizienzen, die im Rahmen privater Verhandlungen bestehen bleiben. Unter diesem Aspekt erscheint es selbst bei Externalitätsproblemen mit wenigen Beteiligten, die den eigentlichen Anwendungsbereich für Verhandlungen darstellen, nicht empfehlenswert, die Individuen vollständig sich selbst zu überlassen.

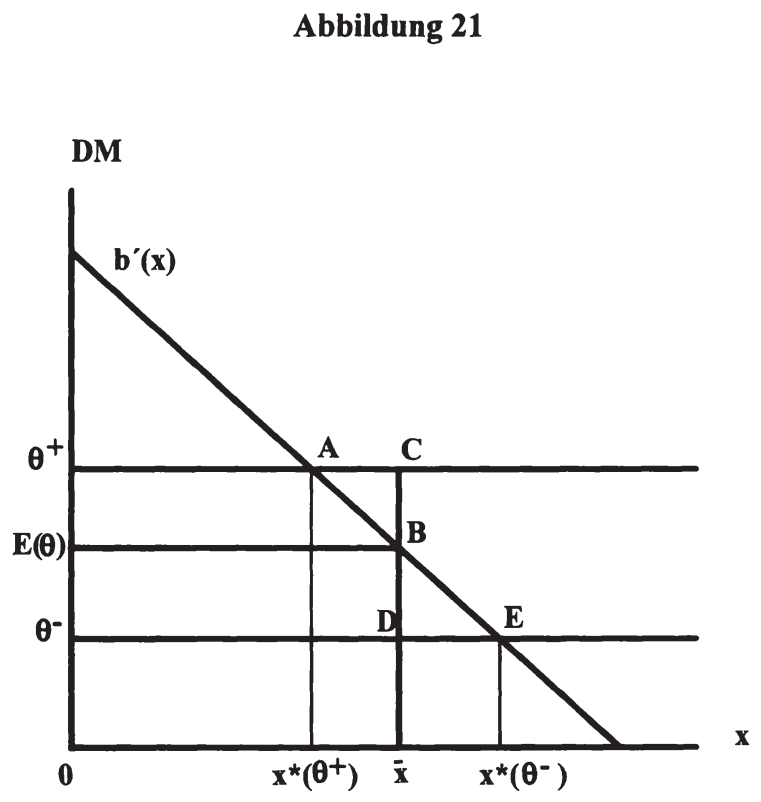


Ein sinnvoller Lösungsansatz könnte auf einem Mittelweg liegen. Verhandlungen und Staatseingriffe müssen sich ja nicht unbedingt ausschließen sondern können einander sinnvoll ergänzen. Wenn beispielsweise eine Zentralinstanz auf der Basis allgemein zugänglicher Informationen über Grenzkosten und Grenznutzen, die mit der Ausübung des externen Effekts verbunden sind, einen durchschnittlich optimalen Standard festlegt, so findet zunächst immerhin eine teilweise Internalisierung statt. Bei dem Externalitätsniveau, das dieser Standard vorschreibt, muß es aber nicht bleiben, da die Beteiligten jederzeit freiwillig in Verhandlungen eintreten und ein Arrangement treffen können, das für jeden vorteilhaft ist. Falls es zu Verhandlungen kommt, sollte sich der Staat dann aber auch zurückhalten und nicht auf einer Einhaltung der Vorschriften bestehen. $\mathrm{DaB}$ sich eine Synthese von Staatseingriffen und Verhandlungen als sehr wirkungsvoll erweisen kann, zeigt sich an einem Beispiel, das in leichter Abwandlung von Illing (1992) übernommen wurde. Betrachten wir dazu die spezielle Version unseres einfachen Externalitätenmodells, die in Abbildung 21 dargestellt ist.

Wir nehmen zur Vereinfachung an, daß der Grenzschaden des Geschädigten konstant sei und jeweils mit einer bestimmten Wahrscheinlichkeit nur die Werte $\theta^{+}$ und $\theta^{-}$annehmen könne. Die Grenzgewinnfunktion $b^{\prime}(x)$ sei bekannt. In dieser Situation könnte der Staat den durchschnittlich optimalen Standard $\bar{x}$ mit $b^{\prime}(\bar{x})=E(\theta)$ als Richtlinie fixieren, gleichzeitig aber Abweichungen nach oben oder unten zulassen, sofern sich die Beteiligten vertraglich darauf einigen. Wenn der Schädiger wieder das Vertragsangebot unterbreiten kann, wird er wie folgt vorgehen: Er wird dem $\theta^{+}$-Typ eine Reduktion des Outputs auf $\mathrm{x}^{*}\left(\theta^{+}\right)$gegen eine Kompensationszahlung in Höhe von $\theta^{+}\left[\overline{\mathrm{x}}-\mathrm{x}^{*}\left(\theta^{+}\right)\right]$anbieten, während er $\operatorname{dem} \theta^{-}$-Typ vorschlagen wird, die Produktion auf $\mathrm{x}^{*}\left(\theta^{-}\right)$zu erhöhen und ihm als Entschädigung den Betrag $\theta^{-}\left[\mathrm{x}^{*}\left(\theta^{-}\right)-\overline{\mathrm{x}}\right] \mathrm{zu}$ zahlen. Dieses Angebot ist furr beide Typen individuell rational, da es keine Verschlechterung gegenüber dem Status quo darstellt. Auch die Bedingung der Anreizverträglichkeit ist für beide erfüllt: Würde der $\theta^{+}$-Typ sich für einen $\theta^{-}$-Typ ausgeben, müßte er eine Produktionserhöhung hinnehmen, für die er nicht einmal vollständig entschädigt würde. Wenn der $\theta^{-}$-Typ behaupten würde, er sei ein $\theta^{+}$-Typ, müßte er für eine Reduktion der Produktionsmenge auf $x^{*}\left(\theta^{+}\right)$mehr bezahlen, als sie ihm wert ist. Der Schädiger kann sich also unter diesen Umständen wieder, wie im Fall vollständiger Information, die gesamten Wohlfahrtsgewinne aneignen, die in Abbildung 21 durch die Dreiecke $\mathrm{ABC}$ bzw. BDE dargestellt sind, je nachdem, ob $\operatorname{der} \theta^{+}$- oder $\operatorname{der} \theta^{-}$-Typ auftritt. Die Verhandlungslösung ist hier also trotz unvoll- 
ständiger Information effizient. Hätte der Staat auf die Vorgabe verzichtet oder auf ihrer strikten Einhaltung bestanden, wäre die jeweilige Allokation ineffizient gewesen. ${ }^{6}$

$\mathrm{Da} B$ dieses Resultat nicht überbewertet werden darf, zeigt sich in Demougin und Illing (1991), wo das gleiche Problem für ein Kontinuum von Schadenstypen analysiert wird. Es stellt sich heraus, daß die Fixierung einer Richtlinie auch in diesem Fall wohlfahrtssteigernd wirkt. Dazu muß die Zentralbehörde den Standard $\overline{\mathrm{x}}$ wieder im Intervall $\left[\mathrm{x}^{*}\left(\theta^{+}\right), \mathrm{x}^{*}\left(\theta^{-}\right)\right]$festsetzen. In diesem Intervall existiert ein optimales $\overline{\mathbf{x}}$ für das die gesamtwirtschaftliche Wohlfahrt ihr Maximum erreicht. Ob dieses $\overline{\mathrm{x}}$ allerdings dem durchschnittlich optimalen Externalitätsniveau entspricht, ist nicht klar. Jedenfalls kann eine für alle Typen ex post effiziente Allokation bei stetiger Wahrscheinlichkeitsverteilung von $\theta$ nicht erreicht werden. Nur für den $\theta^{-}$- und $\operatorname{den} \theta^{+}$- Typ sowie für einen Typ $\theta^{*}(\bar{x})$, für den der gewählte Standard zufällig effizient ist, wird das pareto-optimale Externalitätsniveau realisiert.

${ }^{6}$ Zur Ineffizienz der Verhandlungslösung bei diskreten Schadenstypen vgl. Buchholz und Haslbeck (1991/1992) bzw. Illing (1992). 


\section{Zusammenfassung und Bewertung der Ergebnisse}

Thema dieser Arbeit war die Gegenüberstellung von privaten Verhandlungen und zentral gesteuerten Maßnahmen zur Internalisierung externer Effekte, wenn über den Grenznutzen bzw. die Grenzkosten, die mit ihrer Ausübung verbunden sind, unvollständige Information herrscht. Ausgangspunkt der Überlegungen war der Eindruck, daß Aussagen über die Vorteilhaftigkeit des einen oder des anderen institutionellen Rahmens vor dem Hintergrund der Argumente im Rahmen der traditionellen Pigou-Coase-Kontroverse kaum möglich sind. In der einfachen Welt der Standard-Lehrbücher führen beide Lösungsansätze zur vollständigen Internalisierung, während bei etwas realistischerer Betrachtung weder Verhandlungen noch staatliche Interventionen vollkommen effiziente Allokationen hervorbringen.

Da offenbar beide Internalisierungsansätze Mängel aufweisen, ist es erforderlich, das Ausmaß der jeweils auftretenden Ineffizienzen zu vergleichen, um zu einem Urteil darüber zu gelangen, welche Form der Internalisierung sich als überlegen erweist. Dabei erscheint es sinnvoll, beide Alternativen in einem einheitlichen Modellrahmen zu betrachten und sich auf eine bestimmte Ursache für ihr Versagen zu konzentrieren. Auf diese Weise können deren Auswirkungen auf die Effizienz von Verhandlungen bzw. Staatseingriffen isoliert von anderen Einflüssen herausgearbeitet werden. Unter der Vielzahl vorstellbarer Effizienzhindernisse ist unvollständige Information über Grenznutzen und Grenzkosten der Externalität sicherlich von herausragender Bedeutung. Dies ist offensichtlich, was eine zentrale Regulierung mittels Pigou-Steuern oder Auflagen betrifft. Aber auch bei Verhandlungen beeinträchtigt unvollständige Information über die Zahlungsbereitschaften der Individuen für eine Erhöhung bzw. Senkung des Externalitätsniveaus die Effizienz der Verhandlungsergebnisse. Dies zu zeigen war das Anliegen des ersten Teils dieser Arbeit.

In Kapitel 2 wurde zunächst für den in der Literatur überwiegend diskutierten Standardfall einer rein bilateralen Beziehung zwischen dem Verursacher eines negativen externen Effekts und einem Geschädigten ein Verhandlungsmodell mit vollständiger Information vorgestellt. Dieses wegen seiner Einfachheit häufig verwendete Modell ist dadurch charakterisiert, daß einer der beiden Verhandlungspartner ein ultimatives Vertragsangebot zur Internalisierung des externen Effekts unterbreiten kann, das der andere nur annehmen oder ablehnen kann. Die spezielle Struktur dieses Verhandlungsprozesses ermöglicht es der offerierenden Partei, ihren 
Opponenten auf dessen Reservationsnutzenniveau zu drücken und sich den gesamten Internalisierungsgewinn anzueignen. Unter diesen Bedingungen ist das Verhandlungsergebnis effizient, unabhängig davon, welche Partei offeriert, und unabhängig von der gültigen "Eigentumsordnung" (Verursacher- oder Laisser-FairePrinzip).

Im nächsten Schritt wurde unvollständige Information ins Modell eingeführt. Bei Zugrundelegung des Verursacherprinzips wurde angenommen, der Schädiger habe das Vorschlagsrecht, könne dabei aber die Schadensfunktion des Geschädigten nicht beobachten. Gesucht war das optimale Vertragsangebot, das der Schädiger in dieser Situation unterbreiten würde. Die allgemeinen Rahmenbedingungen des Modells legten es nahe, das Entscheidungsproblem des Schädigers als typisches "Mechanism-Design"-Problem mit einem Agenten aufzufassen. Da die methodischen Grundlagen dieses Lösungsansatzes in der Literatur meist auf mathematisch sehr abstraktem Niveau dargestellt werden, erschien es geboten, die Formalismen etwas ausführlicher zu erläutern und v.a. durch zusätzliche ökonomische Interpretationen zu ergänzen.

In dem betrachteten Modell resultierte die Ineffizienz des Verhandlungsergebnisses daraus, daß dem Geschädigten ein Transfer gezahlt werden muß, der einen reinen Schadensersatz übersteigt. Diese Notwendigkeit ist dadurch begründet, da $\beta$ der Verursacher zum Zeitpunkt seiner Offerte nicht weiß, welchen Schaden seine Aktivität wirklich anrichtet. Der Geschädigte kann sich unter diesen Umständen durch die bewußte Verschleierung seines Schadens eine sog. "Informationsrente" sichern. Er wird deshalb seinen wahren Schaden nur preisgeben, wenn sich der Schädiger von vornherein vertraglich dazu verpflichtet, ihm diese Informationsrente auch bei korrekter Offenbarung in Form einer Zusatzzahlung in entsprechender Höhe zu erhalten. Durch diese Zusatzzahlung wird ein Keil zwischen die einzel- und gesamtwirtschaftlichen Grenzkosten einer Ausdehnung des Externalitätsniveaus getrieben. Dies führt dazu, daß das Aktivitätsniveau, für das der Schädiger sich entscheidet, im Vergleich zum Paretooptimum zu niedrig ist.

Die abschließende Diskussion der Modellannahmen machte deutlich, daß in Gegenwart unvollständiger Information auch unter anderen als den explizit analysierten Rahmenbedingungen mit suboptimalen Verhandlungsergebnissen zu rechnen ist.

In Kapitel 3 wurde der Frage nachgegangen, wie sich eine Erhöhung der Anzahl der Verhandlungsteilnehmer auf die Effizienz des Verhandlungsergebnisses 
auswirkt. Diese Frage stellte sich vor dem Hintergrund einer Standardargumentation für Märkte, auf denen private Güter gehandelt werden. Dieser Argumentation zufolge wirkt sich eine erhöhte Anzahl von Marktteilnehmern effizienzsteigernd aus, weil mit dem Einfluß eines einzelnen Individuums auf den Gleichgewichtspreis auch seine Anreize zur Präferenzverschleierung schwinden. Es wurde davon ausgegangen, daß dieses Argument nicht auf Internalisierungsverhandlungen übertragbar ist, da die meisten Arten externer Effekte aus der Sicht der Geschädigten die Eigenschaften eines öffentlichen Gutes aufweisen. Aus diesem Grund wurde das Verhandlungsmodell des zweiten Kapitels auf ein Szenario mit mehreren Geschädigten erweitert, wobei am Verursacherprinzip und am Vorschlagsrecht des Schädigers festgehalten wurde. Durch diese Erweiterung wurde aus dem ursprünglichen Mechanism-Design-Problem mit einem Agenten eines mit mehreren Agenten, die in einem Spiel mit unvollständiger Information über die Schäden ihrer Rivalen ihren eigenen Schadenskoeffizienten angeben müssen. Das Entscheidungsproblem des Schädigers in diesem modifizierten Modell bestand darin, den gewinnmaximalen "Mechanismus" zu ermitteln, in dem die Angabe des wahren Schadens durch alle Geschädigten ein Bayesiansiches Gleichgewicht bildet. Es zeigte sich, daß auch hier wieder Informationsrenten das Zustandekommen pareto-optimaler Allokationen verhindern.

Die Auswirkungen einer Erhöhung der Anzahl der Geschädigten wurden dann auf der Grundlage eines Modells von Rob (1989) analysiert, das als vereinfachte Version des aus der Erweiterung hervorgegangenen allgemeinen Modells interpretiert werden kann. Anhand dieses Spezialfalls wurde zunächst die Funktionsweise des optimalen Mechanismus für den einfachen Fall zweier Geschädigter erläutert. Dabei wurde zur Unterstützung eine spezielle graphische Darstellungsmethode herangezogen, mit deren Hilfe v.a. das strategische Verhalten der Geschädigten und das dabei auftretende Problem multipler Gleichgewichte veranschaulicht werden konnte. Schließlich wurde untersucht, wie sich die Effizienz des Verhandlungsergebnisses in Robs Modell verändert, wenn die Anzahl der Geschädigten gegen Unendlich geht. Es stellte sich heraus, daß die Ineffizienzen dramatisch zunehmen.

Dieses Resultat konnte intuitiv durch eine Art "informationelles Trittbrettfahrerproblem" erklärt werden: Bei einer Vielzahl von Geschädigten hat die Schadensmeldung eines einzelnen aus seiner Sicht so gut wie keine Auswirkungen auf die Produktionsentscheidung des Schädigers. Der Anreiz, den Schaden zu übertreiben, ist in diesem Fall höher als bei einer geringen Anzahl von Geschädigten, wo jeder 
damit rechnen muß, daß der Schädiger sein Aktivitätsniveau und damit die Transferzahlungen umso stärker einschränkt, je höher die Schadensmeldungen sind. Die Vielzahl der Geschädigten treibt also die Pro-Kopf-Kosten der Informationsenthüllung in die Höhe und verschärft die Ineffizienz der Verhandlungslösung. Durch dieses Ergebnis konnte die in der Literatur vorherrschende Ansicht, daß private Internalisierungsverhandlungen in "großen Gruppen" aufgrund von Free-Rider-Problemen keine Erfolgsaussichten haben können, auch aus informationsökonomischer Sicht bestätigt werden.

Nicht zuletzt aufgrund dieses Ergebnisses wurde im zweiten Teil der Arbeit, der sich mit staatlichen Internalisierungsmaßnahmen bei unvollständiger Information befaßt, die Gegenüberstellung mit der Verhandlungslösung auf das bilaterale Externalitätenmodell beschränkt. Die Analyse des Falls mit vielen Beteiligten, insbesondere mit vielen Geschädigten, läßt keine zusätzlichen Erkenntnisse erwarten, da Verhandlungen in einem Vergleich mit zentralen Regulierungsmaßnahmen, wenn überhaupt, dann nur bei Externalitätsproblemen mit wenigen Beteiligten bestehen können.

In Kapitel 4 wurden zunächst die Informationsprobleme des Staates bei der Ausgestaltung traditioneller Lenkungsinstrumente wie Pigou-Steuern und Auflagen herausgearbeitet. Es wurden die Anreize untersucht, die Schädiger und Geschädigten dazu bewegen, ihre Grenzgewinne bzw. Grenzkosten falsch anzugeben, wenn sie wissen, welche Regulierungsmaßnahme eingesetzt werden soll. Es wurde gezeigt, daß es durch bewußte "Präferenzverschleierung" zu Fehlallokationen kommt. Dadurch konnte der Bedarf an geeigneten Informationsenthüllungsverfahren begründet werden.

Eine überblicksartige Diskussion solcher Verfahren war Gegenstand des fünften Kapitels. Dabei wurden die gängigen Präferenzenthüllungsmechanismen, die ursprünglich als "theoretisch ideale" Lösungen des Informationsproblems bei der optimalen Bereitstellung öffentlicher Güter entwickelt wurden, auf das Externalitätenmodell übertragen. Der Schwerpunkt des Kapitels wurde auf die kritische Beurteilung dieser Mechanismen gelegt, die in der Literatur nicht immer ausreichende Beachtung findet. Es sollten v.a. die verschiedenen "Unmöglichkeitstheoreme", die die Schwächen dieser Konzepte offenbaren, in einem logischen Zusammenhang präsentiert werden. Es wurde dabei auf die technisch meist recht überfrachteten Beweise dieser Theoreme verzichtet. Stattdessen wurde versucht, deren Konsequen- 
zen für die Anwendbarkeit von Präferenzenthüllungsmechanismen speziell auf Externalitätenprobleme anhand einfacher Beispiele zu demonstrieren.

Die gewonnenen Erkenntnisse legten den Schluß nahe, daß der Einsatz dieser Verfahren nicht nur aus Gründen der Praktikabilität fragwürdig ist. Die Hauptkritik aus theoretischer Sicht bezog sich auf die weitgehende Unmöglichkeit der Durchsetzung echter Pareto-Verbesserungen. Bei den Groves-Mechanismen wird diese durch staatliche Budgetdefizite oder -überschüsse verhindert, die gerade im Fall "kleiner Gruppen" nicht durch eine anreizneutrale Besteuerung bzw. Subventionierung der beteiligten Individuen ausgeglichen werden können. Der AGV-Mechanismus ist zwar haushaltsneutral, dafür sind aber seine Verteilungswirkungen extrem unausgewogen, so daß manche Individuen im Falle einer Anwendung zur Teilnahme gezwungen werden müßten, weil sie nicht einmal ihren Reservationsnutzen, geschweige denn einen Anteil an den Internalisierungsgewinnen erhalten würden. Diese und andere theoretische Schwächen von Präferenzenthüllungsmechanismen führten zusammen mit verschiedenen praktischen Einwänden letztendlich zu ihrer Ablehnung als zufriedenstellende Internalisierungsinstrumente.

In Kapitel 6 wurde auf der Basis der Einsichten des vorangegangenen Kapitels schließlich der Frage nachgegangen, wie einfachere Internalisierungsmaßnahmen, die zwar auf die Enthüllung privater Informationen verzichten und deshalb von vornherein ineffizient, dafür aber frei von den Mängeln der Präferenzenthüllungsmechanismen sind, im Vergleich zur Verhandlungslösung abschneiden. Anhand eines speziellen Beispiels konnte gezeigt werden, daß durch die wohlüberlegte Wahl zwischen einer Pigou-Steuer oder einer Auflage, die die gesamtwirtschaftliche Wohlfahrt nur im statistischen Durchschnitt über die privaten Informationen maximiert, durchaus höhere Wohlfahrtsgewinne erzielt werden können als in Verhandlungen. Zuletzt wurde deutlich gemacht, da $\beta$ Staatseingriffe und Verhandlungen sich unter Umständen sinnvoll ergänzen können.

Am Ende dieser Arbeit sollte der Versuch einer Beantwortung einer der zentralen finanzwissenschaftlichen Fragestellungen stehen: Lassen sich durch unvollständige Information Staatseingriffe zur Internalisierung externer Effekte begründen?

Nach der traditionellen Argumentation der Verfechter des Property-Rights-Ansatzes liefern Internalisierungsverhandlungen in Abwesenheit von Transaktionskosten zumindest in Situationen mit wenigen Beteiligten effiziente Ergebnisse. Es sei demnach müßig, nach Alternativen Ausschau zu halten, die im günstigsten Fall die 
gleiche Eigenschaft hätten, wahrscheinlich aber zu suboptimalen Allokationsergebnissen führen würden. Unter diesen Umständen bestehe kein Grund, staatliche Interventionen zu fordern.

Die Berücksichtigung unvollständiger Information ändert zunächst einmal den Ausgangspunkt dieses Arguments. Es hat sich gezeigt, da $\beta$ Verhandlungen in diesem Fall keine First-Best-Lösungen zustande bringen. Dies liegt daran, daß private Information den Individuen eine gewisse Macht verleiht, die sie dazu benützen, sich bei der Verteilung der erzielbaren Internalisierungsgewinne einen möglichst hohen Anteil zu verschaffen. Durch die Verschleierung ihrer Präferenzen können sie sich Informationsrenten sichern, auf die sie grundsätzlich nicht verzichten werden. Diese Renten stellen aus einzelwirtschaftlicher Perspektive einen Kostenfaktor dar, den die Privaten in ihren Entscheidungskalkülen berücksichtigen. Diese Kosten können dadurch begrenzt werden, daß der externe Effekt nur teilweise internalisiert wird. Gemessen an der pareto-optimalen Allokation liegt also ein "Marktversagen" vor.

$\mathrm{Ob}$ allein dadurch schon Staatseingriffe begründet werden können hängt davon $a b$, inwieweit eine Zentralinstanz in der Lage ist, effiziente Allokationen zu implementieren. Auf den ersten Blick scheint diese Möglichkeit zu bestehen. Die Enthüllung privater Information ist zwar auch für den Staat nicht kostenlos, da die Individuen bei zentralen Internalisierungsmaßnahmen ebenso Anreize zur Präferenzverschleierung haben wie bei Verhandlungen. Da der Staat aber annahmegemäß keine eigennützigen Motive verfolgt, hat er, anders als die privaten Wirtschaftseinheiten, kein grundsätzliches Interesse daran, die Informationsrenten zu begrenzen. Er könnte deshalb die Kosten, die nötig wären, um eine vollständige Internalisierung des externen Effekts durchzusetzen, im Prinzip übernehmen. Er müßte dazu nur einen Präferenzenthüllungsmechanismus konzipieren, bei dessen Anwendung den Individuen ihre Informationsrenten in Form von Subventionen in entsprechender Höhe erhalten bleiben. Das Problem dabei ist, daß dazu Mittel erforderlich sind, die durch Besteuerung der Privaten aufgebracht werden müssen. Dabei könnten prinzipiell auch Individuen zu Zahlungen herangezogen werden, die mit dem Externalitätsproblem eigentlich nichts zu tun haben. In diesem Fall könnten einerseits steuerliche Zusatzlasten auftreten, die die Internalisierungsgewinne teilweise wieder zunichte machen würden. Andererseits ist eine Belastung Unbeteiligter auch unter Verteilungsgesichtspunkten nicht unproblematisch. Wenn man aus Gerechtigkeitsgründen vermeiden will, daß Außenstehende besteuert werden, bleibt letztendlich 
doch wieder keine andere Wahl, als die Kosten der Informationsenthüllung den Beteiligten selbst aufzubürden.

Wie Kapitel 5 gezeigt hat, ist aber ein "interner" Budgetausgleich generell unmöglich, wenn aus Gründen der "Implementationssicherheit" für alle Individuen die Offenbarung ihrer wahren Präferenzen dominante Strategie sein soll. In diesem Fall bleiben immer Mittel übrig, die nicht zurücktransferiert werden können, ohne die ursprünglich beabsichtigten Enthüllungsanreize zu zerstören. Ein Budgetüberschuß könnte zwar an Dritte ausgeschüttet werden, für die Betroffenen ergäbe sich aber ein Wohlfahrtsverlust, der durchaus höher sein kann als der, der bei privaten Verhandlungen auftritt. In dieser Situation ist es sehr fraglich, ob die Individuen einen solchen Mechanismus als Alternative zu freiwilligen Verhandlungen überhaupt akzeptieren würden.

Ein Budgetausgleich ist nur möglich, wenn man auf dominante Strategien verzichtet. In diesem Fall steigt einerseits das Risiko, das Ziel der Präferenzenthüllung am Ende doch zu verfehlen. Falls es andererseits im Gleichgewicht trotz dieses Risikos zur Enthüllung kommen sollte, muß damit gerechnet werden, daß einige Individuen trotz Internalisierung des externen Effekts unter ihrem Reservationsnutzenniveau bleiben werden. Derartig unausgewogene Verteilungswirkungen erschweren die Durchsetzung dieser Mechanismen. Die bloße Implementation des Externalitätsniveaus, bei dem Grenznutzen und Grenzkosten übereinstimmen, reicht eben nicht aus. Sie verliert für die Beteiligten umso mehr an Bedeutung, je mehr sie ihren Gerechtigkeitsvorstellungen widerspricht.

Die theoretischen Mängel von Präferenzenthüllungsmechanismen liegen also auch in ihren Verteilungswirkungen begründet. Diesem Umstand wird in der Literatur kaum Beachtung geschenkt. Wer sich von Verteilungsargumenten nicht überzeugen läßt, wird zumindest zugeben müssen, daß Präferenzenthüllungsverfahren viel zu hohe Ansprüche an die Privaten und den Staat stellen, um im Hinblick auf praktische Anwendungen wirklich ernst genommen zu werden. Eine Politik der "Daumenregeln", wie sie in Kapitel 6 beschrieben wurde, entspricht wohl viel eher den Vorstellungen von einer realistischen Staatstätigkeit als die etwas "abgehobenen" Enthüllungsmechanismen. Damit dürfte aber klar sein, daß man auch von Staatseingriffen keine vollkommen effizienten Allokationsergebnisse erwarten darf.

Spricht diese Erkenntnis nun für oder gegen Staatseingriffe? Das Rechenbeispiel in Kapitel 6 hat zwar gezeigt, da $B$ unter bestimmten Voraussetzungen einfache Internalisierungsmaßnahmen zu höheren Wohlfahrtsgewinnen führen können als 
Verhandlungen. Unter anderen Bedingungen könnte sich aber auch das Gegenteil herausstellen. Die unbedingte Überlegenheit von Staatseingriffen kann durch solche Beispiele zwar nicht nachgewiesen werden. Die Einfachheit der untersuchten staatlichen Maßnahmen wirft aber doch ein recht schlechtes Licht auf die Verhandlungslösung.

Richter und Wiegard (1993) beurteilen deshalb die Bedeutung des Informationsproblems für die theoretische Begründung von Staatseingriffen wie folgt: "Bei asymmetrischer Information sind im allgemeinen Spielräume für effizienzsteigernde Staatseingriffe vorhanden. Allerdings kann man daraus noch kein unbedingtes Plädoyer für staatliche Maßnahmen ableiten. Unangenehm ist (...), daß über die Vorteilhaftigkeit von Staatseingriffen nicht parameterfrei entschieden werden kann." Eine theoretisch überzeugende Begründung stehe also auch bei asymmetrischer Information noch aus. ${ }^{1}$

Trotz dieser grundsätzlich zutreffenden Einschätzung kann man aber zumindest behaupten, da $\beta$ die Position der Befürworter staatlicher Internalisierungsmaßnahmen durch die Betrachtung unvollständiger Information gestärkt wird. Die ursprünglichen Vorteilhaftigkeitsrelationen haben sich im Vergleich zur Situation mit vollständiger Information eindeutig zugunsten des Pigou-Ansatzes verschoben: Staatliche Maßnahmen können in der Gegenüberstellung mit Verhandlungen nicht mehr nur gleichziehen, sondern sich trotz einfachster Ausgestaltung fallweise sogar als überlegen erweisen. Der Zwang zur Begründung von Staatseingriffen wird dadurch deutlich abgeschwächt. Man könnte jetzt sogar den Spieß umdrehen und eine Begründung dafür verlangen, warum man auf Staatseingriffe zugunsten der "Marktlösung" verzichten sollte. Eine definitive Antwort auf die Frage, welchem der beiden Internalisierungsansätze der Vorzug zu geben ist, kann letztendlich nicht gegeben werden. Überraschend ist allerdings die Tatsache, daß das Informationsproblem ausgerechnet zu einer relativen Aufwertung staatlicher Maßnahmen führt. Diesbezüglich hätte man wohl eher das Gegenteil erwartet.

Wenn man etwas pragmatischer eingestellt ist, wird man ohnehin keine theoretisch einwandfreie Begründung von Staatseingriffen verlangen. Man wird stattdessen den akuten umweltpolitischen Handlungsbedarf erkennen und sich auf den Standpunkt stellen, daß die wichtigsten Probleme unserer Zeit Externalitätsprobleme mit vielen Beteiligten sind, für die Verhandlungen sowieso nicht zur Diskussion stehen. Darüber hinaus wird man darauf verweisen, daß Verhandlungen innerhalb

\footnotetext{
${ }^{1}$ Siehe Richter und Wiegard (1993, S. 198).
} 
eines Landes selbst in Situationen mit wenigen Beteiligten empirisch kaum eine Rolle spielen dürften. Wirklich bedeutsam werden Internalisierungsverhandlungen erst bei der Lösung grenzüberschreitender Umweltprobleme, da hier keine überstaatliche Regierung existiert, die Regulierungsmaßnahmen vorschreiben könnte. Wenn man allerdings die Ergebnisse solcher Verhandlungen betrachtet, hat man nicht unbedingt den Eindruck, daß sie erfolgreich waren. Als Paradebeispiel hierfür kann der sog. "Umweltgipfel" von Rio im Jahre 1992 angefürt werden. In Verbindung mit anderen Gründen, zu denen sicher in erster Linie die Wahrung nationaler Interessen zählt, mag auch asymmetrische Information für dessen Scheitern mitverantwortlich gewesen sein.

Wenn eine übergeordnete Zentralinstanz zur Verfügung steht, erscheint es insgesamt doch eher sinnvoll, auf Staatseingriffe zu bauen. Für Richter und Wiegard (1993) besteht kein Zweifel daran, "daß der Staat in vielen Fällen über Gebührenoder Steuererhebung, Umweltstandards oder Verkauf von Emissionszertifikaten aktiv werden sollte."2 Diese Arbeit sollte gezeigt haben, daß diese Ansicht nicht unbegründet ist. Wenn man der gleichen Meinung ist, wird man als Ökonom seine künftigen Forschungsanstrengungen auf die Optimierung des bewährten umweltpolitischen Instrumentariums unter Berücksichtigung des Informationsproblems konzentrieren, sich dabei aber von den Irrwegen, die die Diskussion der Präferenzenthüllungsverfahren aufgezeigt hat, fernhalten.

2Siehe Richter und Wiegard (1993, S. 198 f.). 


\section{Anhang A}

\section{Herleitung von Gleichung (3.17)}

Die Gewinnfunktion des Schädigers lautet

$$
\text { (A.1) } E(\pi)=\int_{T} b(x(\theta))-\sum_{i=1}^{n} z_{i}(\theta) f(\theta) d \theta
$$

Nach Vertauschen von Summation und Integration beim zweiten Term auf der rechten Seite von (A.1) und Erweiterung mit $\sum_{i=1}^{n} \int_{T} \theta_{i} x(\theta) f(\theta) d \theta$ erhält man

$$
\text { (A.2) } \begin{aligned}
E(\pi)= & \int_{T} b(x(\theta)) f(\theta) d \theta- \\
& \sum_{i=1}^{n} \int_{T} z_{i}(\theta)-\theta_{i} x(\theta) f(\theta) d \theta- \\
& \sum_{i=1}^{n} \int_{T} \theta_{i} x(\theta) f(\theta) d \theta .
\end{aligned}
$$

Berücksichtigt man in Gleichung (A.2), daß per Definition von $\mathrm{u}_{\mathrm{i}}\left(\theta_{\mathrm{i}}\right)$ die Beziehung $\int_{T} z_{i}(\theta)-\theta_{i} x(\theta) f(\theta) d \theta=\int_{\theta_{i}^{-}}^{\theta_{i}^{+}} u_{i}\left(\theta_{i}\right) f_{i}\left(\theta_{i}\right) d \theta_{i}$ gilt und eliminiert anschließend $u_{i}\left(\theta_{i}\right)$ gemäß Gleichung (3.16), dann ergibt sich

$$
\text { (A.3) } \begin{aligned}
E(\pi)= & \int_{T} b(x(\theta)) f(\theta) d \theta- \\
& \sum_{i=1}^{n} u_{i}\left(\theta_{i}^{+}\right) \int_{\theta_{i}^{-}}^{\theta_{i}^{*}} f_{i}\left(\theta_{i}\right) d \theta_{i}-\sum_{i=1}^{n} \int_{\theta_{i}^{-}}^{\theta_{i}^{+}} \int_{\theta_{1}}^{\theta_{i}^{*}} E_{i} x\left(t_{i}\right) d t_{i} f_{i}\left(\theta_{i}\right) d \theta_{i}- \\
& \sum_{i=1}^{n} \int_{T} \theta_{i} x(\theta) f(\theta) d \theta .
\end{aligned}
$$

Nach Berücksichtigung von $\int_{\theta_{i}^{-}}^{\theta_{i}^{+}} f_{i}\left(\theta_{i}\right) d \theta_{i}=1$ und Vertauschung der Integrationsreihenfolge beim Doppelintegral im dritten Term auf der rechten Seite von (A.3) folgt 


$$
\text { (A.4) } \begin{aligned}
E(\pi)= & \int_{T} b(x(\theta)) f(\theta) d \theta- \\
& \sum_{i=1}^{n} u_{i}\left(\theta_{i}^{+}\right)-\sum_{i=1}^{n} \int_{\theta_{i}^{i}}^{\theta_{i}^{+}} E_{i} x\left(t_{i}\right) \int_{\theta_{i}^{-}}^{t_{i}} f_{i}\left(\theta_{i}\right) d \theta_{i} d t_{i}- \\
& \sum_{i=1}^{n} \int_{T} \theta_{i} x(\theta) f(\theta) d \theta .
\end{aligned}
$$

Den dritten Term dieser Gleichung kann man weiter umformen, indem man zunächst beachtet, $d a ß \int_{\theta_{i}^{-}}^{t_{1}} f_{i}\left(\theta_{i}\right) d \theta_{i}=F_{i}\left(t_{i}\right)$ ist. Dann benennt man die verbleibende Integrationsvariable $t_{i}$ um in $\theta_{i}$ und setzt für den Erwartungswert $E_{i} x\left(\theta_{i}\right)$ die Definition (3.13) ein. Schließlich erweitert man mit $f_{i}\left(\theta_{i}\right)$, so daß Gleichung

$$
\text { (A.5) } \begin{aligned}
E(\pi)= & \int_{T} b(x(\theta)) f(\theta) d \theta- \\
& \sum_{i=1}^{n} u_{i}\left(\theta_{i}^{+}\right)-\sum_{i=1}^{n} \int_{\theta_{i}^{i}}^{\theta_{i}^{*}} \int_{T_{-i}} x(\theta) f_{-i}\left(\theta_{-i}\right) d \theta_{-i} \frac{F_{i}\left(\theta_{i}\right)}{f_{i}\left(\theta_{i}\right)} f_{i}\left(\theta_{i}\right) d \theta_{i} \\
& \sum_{i=1}^{n} \int_{T} \theta_{i} x(\theta) f(\theta) d \theta
\end{aligned}
$$

resultiert. Daraus ergibt sich nach geeigneter Zusammenfassung, erneuter Vertauschung der Reihenfolge von Summation und Integration in den letzten beiden Termen und Ausklammern von $\mathbf{x}(\theta)$ Gleichung (3.17). 


\section{Anhang B}

Berechnung von $\lim _{n \rightarrow \infty} \omega_{2}(\mathbf{n})$.

Es ist zu zeigen, daß

(B.1) $\lim _{n \rightarrow \infty} \omega_{2}(n)=P\left(2 \sum_{i=1}^{n} \theta_{i} \leq \beta n / \sum_{i=1}^{n} \theta_{i} \leq \beta n\right)=\frac{P\left(2 \sum_{i=1}^{n} \theta_{i} \leq \beta n\right)}{P\left(\sum_{i=1}^{n} \theta_{i} \leq \beta n\right)}=0$.

Die Schadensparameter $\theta_{i}$ sind annahmegemäß identisch und unabhängig verteilte Zufallsvariablen. Nach dem zentralen Grenzwertsatz konvergiert die Wahrscheinlichkeitsverteilung einer Summe solcher Zufallsvariablen für $n \rightarrow \infty$ gegen die Normalverteilung. Zur Bestimmung der Wahrscheinlichkeiten in Zähler und Nenner von (B.1) müssen die Zufallsvariablen $\sum \theta_{i}$ bzw. $2 \sum \theta_{i}$ zunächst standardisiert werden, indem man ihren jeweiligen Erwartungswert subtrahiert und sie anschlieBend durch ihre Standardabweichung dividiert. $\mathrm{Da} \theta_{\mathrm{i}}$ im Intervall $[0 ; 1]$ gleichverteilt ist, ist $\mathrm{E}\left(\theta_{\mathrm{i}}\right)=1 / 2$ und $\operatorname{Var}\left(\theta_{\mathrm{i}}\right)=1 / 12$. Nach den Rechenregeln für Erwartungswerte und Varianzen von Summen unabhängig und identisch verteilter Zufallsvariablen resultiert $E\left(\sum_{i=1}^{n} \theta_{i}\right)=n / 2$ und $E\left(2 \sum_{i=1}^{n} \theta_{i}\right)=n$ sowie $\operatorname{Var}\left(\sum_{i=1}^{n} \theta_{i}\right)=n / 12$ und $\operatorname{Var}\left(2 \sum_{\mathrm{i}=1}^{\mathrm{n}} \theta_{\mathrm{i}}\right)=\mathrm{n} / 3$. Für hinreichend großes $\mathrm{n}$ gilt nach Standardisierung

$$
\mathrm{P}\left(\sum_{i=1}^{\mathrm{n}} \theta_{i} \leq \beta \mathrm{n}\right)=\mathrm{P}\left(\frac{\sum_{\mathrm{i}=1}^{\mathrm{n}} \theta_{\mathrm{i}}-\mathrm{n} / 2}{\sqrt{\mathrm{n} / 12}} \leq \frac{\beta \mathrm{n}-\mathrm{n} / 2}{\sqrt{\mathrm{n} / 12}}\right) \approx \varphi(\sqrt{\mathrm{n}} \sqrt{12}(\beta-1 / 2))
$$

wobei $\varphi($.$) für die Verteilungsfunktion der Standardnormalverteilung steht. In glei-$ cher Weise erhält man

(B.3) $P\left(2 \sum_{i=1}^{n} \theta_{i} \leq \beta n\right) \approx \varphi(\sqrt{n} \sqrt{3}(\beta-1))$,

so daß sich näherungsweise 
(B.4) $\omega_{2}(\mathrm{n}) \approx \frac{\varphi(\sqrt{\mathrm{n}} \sqrt{3}(\beta-1))}{\varphi(\sqrt{\mathrm{n}} \sqrt{12}(\beta-1 / 2))}$

ergibt. Im nächsten Schritt gilt es, $\lim _{n \rightarrow \infty} \omega_{2}(n)$ zu berechnen. Dieser Grenzwert wird im wesentlichen durch die Höhe des Pro-Kopf-Gewinns $\beta$ bestimmt. $\mathrm{Da} \beta \quad 0$ keinen Sinn ergibt, ist klar. Wäre $\beta \geq 1$, dann wäre unter unseren Voraussetzungen der Gewinn pro Geschädigten größer oder gleich dem maximal möglichen Schaden $\theta^{+}=1$. Nach den Regeln des Mechanismus kann aber die Transferzahlung an einen Geschädigten ebenfalls höchstens $\theta^{+}=1$ betragen, so da $\beta$ der Schädiger selbst im für ihn ungünstigsten Fall, in dem er jedem Geschädigten $\theta^{+}$zu zahlen hätte, Gewinne machen würde. Er würde also die Produktion unter allen Umständen aufnehmen und der interessante Fall, daß nicht produziert wird, obwohl es sinnvoll wäre, könnte gar nicht auftreten. Damit also das hier betrachtete Problem überhaupt ökonomisch interssant ist, muß $0<\beta<1$ sein.

Wegen $\beta<1$ geht nun der Zähler von (B.4) für $n \rightarrow \infty$ sicher gegen 0 , da das Argument von $\varphi($.) immer stärker negativ wird und der Wert der Standardnormalverteilung gegen Null konvergiert. Das Verhalten des Nenners im Unendlichen ist dagegen nicht eindeutig: Ist $\beta>1 / 2$, dann geht das Argument von $\varphi($.) gegen unendlich und $\varphi($.) selbst gegen Eins. Für $\beta=1 / 2$ nimmt der Nenner unabhängig von $n$ den Wert $1 / 2$ an, da $\varphi(0)=1 / 2$ ist. Für $\beta \geq 1 / 2$ konvergiert demnach $\omega_{2}(n)$ gegen Null.

Falls $\beta<1 / 2$ ist, strebt auch der Nenner von von (B.4) gegen Null. Nach Anwendung des Satzes von L'Hôspital kann aber das Verhalten von $\omega_{2}(\mathrm{n})$ eindeutig bestimmt werden. Dieser Satz besagt, daß, wenn

(B.5) $\lim _{n \rightarrow \infty} \varphi(u(n))=\lim _{n \rightarrow \infty} \varphi(v(n))=0$

ist, die Beziehung

(B.6) $\lim _{n \rightarrow \infty} \frac{\varphi(u(n))}{\varphi(v(n))}=\lim _{n \rightarrow \infty} \frac{\varphi^{\prime}(u(n)) u^{\prime}(n)}{\varphi^{\prime}(v(n)) v^{\prime}(n)}$

gilt. Daraus folgt 
(B.7) $\lim _{n \rightarrow \infty} \omega_{2}(n)=\lim _{n \rightarrow \infty} \frac{\varphi(\sqrt{n} \sqrt{3}(\beta-1))}{\varphi(\sqrt{n} \sqrt{12}(\beta-1 / 2))}=$

$$
=\lim _{n \rightarrow \infty} \frac{\varphi^{\prime}(\sqrt{n} \sqrt{3}(\beta-1)) \sqrt{3}(\beta-1)(1 / 2 \sqrt{n})}{\varphi^{\prime}(\sqrt{n} \sqrt{12}(\beta-1 / 2)) \sqrt{12}(\beta-1 / 2)(1 / 2 \sqrt{n})} .
$$

Dabei bezeichnet $\varphi^{\prime}($.) die Dichtefunktion der Standardnormalverteilung. Allgemein gilt für die Dichte einer standardnormalverteilte Zufallsvariable y

(B.8) $\varphi^{\prime}(\mathrm{y})=\frac{1}{\sqrt{2 \pi}} \mathrm{e}^{-\frac{y^{2}}{2}}$.

Wenn man in (B.8) die Werte aus (B.7) einsetzt, erhält man schließlich

$$
\text { (B.9) } \begin{aligned}
\lim _{n \rightarrow \infty} \omega_{2}(n) & =\frac{(\beta-1) \mathrm{e}^{-1 / 2(\sqrt{n} \sqrt{3}(\beta-1))^{2}}}{2(\beta-1 / 2) \mathrm{e}^{-1 / 2(\sqrt{n} \sqrt{12}(\beta-1 / 2))^{2}}}= \\
= & \frac{1}{2} \frac{(\beta-1)}{(\beta-1 / 2)} \mathrm{e}^{-\mathrm{\beta}(3-9-9 / 2)} .
\end{aligned}
$$

Da der Exponent der e-Funktion für $\beta<1 / 2$ insgesamt negativ ist, ist auch in diesem Fall $\lim _{n \rightarrow \infty} \omega_{2}(n)=0$. 


\section{Anhang C}

Ein einfaches Beispiel für die fehlende individuelle Rationalität des AGV-Mechanismus

Der erwartete Gewinn des Schädigers im Enthüllungsgleichgewicht beträgt

$$
\text { (C.1) } \begin{aligned}
& \mathrm{E} \pi(\mathrm{a})=\mathrm{E}_{\theta}\left\{\mathrm{b}\left(\mathrm{x}^{*}, \mathrm{a}\right)-\mathrm{d}\left(\mathrm{x}^{*}, \theta\right)\right\}-\mathrm{E}_{\theta}\left\{\varepsilon_{\mathrm{G}}(\theta)\right\}= \\
& =\mathrm{E}_{\theta}\left\{\mathrm{b}\left(\mathrm{x}^{*}, \mathrm{a}\right)-\mathrm{d}\left(\mathrm{x}^{*}, \theta\right)\right\}-\mathrm{E}_{\theta} \mathrm{E}_{\mathrm{a}}\left\{\mathrm{b}\left(\mathrm{x}^{*}, \mathrm{a}\right)\right\} .
\end{aligned}
$$

Die Grenzgewinnfunktion des Schädigers sei durch $b^{\prime}(x, a)=a-c x$, die Grenzschadensfunktion des Geschädigten durch $\mathrm{d}^{\prime}(\mathrm{x}, \theta)=\theta$ definiert. Daraus folgt

(C.2) $b(x, a)=x(a-c x / 2)$

und

(C.3) $\mathrm{d}(\mathrm{x}, \theta)=\theta \mathrm{x}$

sowie

(C.4) $\mathrm{x}^{*}=(\mathrm{a}-\theta) / \mathrm{c}$.

Setzt man (C.4) in (C.2) und (C.3) ein und bildet die jeweiligen Erwartungswerte, dann erhält man

(C.5) $\mathrm{E}_{\theta}\left\{\mathrm{b}\left(\mathrm{x}^{*}, \mathrm{a}\right)\right\}=(1 / 2 \mathrm{c})\left(\mathrm{a}^{2}-\mathrm{E}\left(\theta^{2}\right)\right)$,

(C.6) $\mathrm{E}_{\theta}\left\{\mathrm{d}\left(\mathrm{x}^{*}, \mathrm{a}\right)\right\}=(1 / 2 \mathrm{c})\left(\mathrm{aE}(\theta)-\mathrm{E}\left(\theta^{2}\right)\right)$ und

(C.7) $\mathrm{E}_{\theta} \mathrm{E}_{\mathrm{a}}\left\{\mathrm{b}\left(\mathrm{x}^{*}, \mathrm{a}\right)\right\}=(1 / 2 \mathrm{c})\left(\mathrm{E}\left(\mathrm{a}^{2}\right)-\mathrm{E}\left(\theta^{2}\right)\right)$.

Nach Aufsummieren der letzten drei Gleichungen ergibt sich

(C.8) $\mathrm{E} \pi(\mathrm{a})=(1 / 2 \mathrm{c})\left(\mathrm{a}^{2}-2 \mathrm{aE}(\theta)-\mathrm{E}\left(\mathrm{a}^{2}\right)+2 \mathrm{E}\left(\theta^{2}\right)\right)$. 
Der AGV-Mechanismus ist nur individuell rational, wenn $E \pi(a) \geq 0$ ist. Nehmen wir beispielsweise an, $\theta$ sei im Intervall $[0,1]$ und a im Intervall [1,2] gleichverteilt. In diesem Fall ist $\mathrm{E}(\theta)=1 / 2, \mathrm{E}\left(\theta^{2}\right)=1 / 3$ und $\mathrm{E}\left(\mathrm{a}^{2}\right)=7 / 3 .{ }^{3}$ Die Bedingung für individuelle Rationalität reduziert sich dann auf

(C.9) $a^{2}-a-5 / 3 \geq 0$.

Diese ist nur dann erfüllt, wenn ungefähr a $\geq 1,88$ ist, d.h. der Mechanismus ist mit Wahrscheinlichkeit $\mathrm{F}_{\mathrm{a}}(1,88)=0,88$ nicht individuell rational. ${ }^{4}$

${ }^{3}$ Bei der Gleichverteilung gilt $E\left(y^{2}\right)=\frac{1}{b-a} \int_{a}^{b} y^{2} d y=\frac{1}{b-a}\left|\frac{1}{3} y^{3}\right|_{a}^{b}$, falls $y \in[a, b]$.

${ }^{4} \mathrm{~F}_{\mathrm{a}}($.$) ist die Wahrscheinlichkeitsfunktion von a.$ 


\section{Literaturverzeichnis}

Adar, Z. und J. M. Griffin, 1976, Uncertainty and the Choice of Pollution Control Instruments, Journal of Environmental Economics and Management 3, S. 178 - 188.

Arrow, K. J., 1951, Social Choice and Individual Values, New York.

Arrow, K. J., 1979, The Property Rights Doctrine and Demand Revelation under Incomplete Information, in: M. J. Boskin (Hrsg.), Economics and Human Welfare Essays in Honor of Tibor Scitovsky, New York u.a.O.

Baron, D. und R. Myerson, 1982, Regulating a Monopolist with Unknown Costs, Econometrica 50, S. 911 - 930.

Baumol, W. J. und W. E. Oates, 1988, The Theory of Environmental Policy, 2. Aufl., Cambridge u.a.O.

Binmore, K., M. J. Osborne und A. Rubinstein, 1992, Non-Cooperative Models of Bargaining, in: R. J. Aumann und S. Hart (Hrsg.), Handbook of Game Theory with Economic Applications Vol. I, Amsterdam u.a.O.

Bohm, P., 1979, Estimating Willingness to Pay: Why and How?, Scandinavian Journal of Economics 81, S. 142 - 153.

Bossert, W. und F. Stehling, 1990, Theorie kollektiver Entscheidungen, Berlin u.a.O.

Buchanan, J. M., 1967, Cooperation and Conflict in Public-Goods Interaction, Western Economic Journal 5, S. 109 - 121.

Buchanan, J. M. und W. C. Stubblebine, 1962, Externality, Economica 29, S. 371 $-384$.

Buchholz, W. und C. Haslbeck, 1991/1992, Private Verhandlungen und staatliche Regulierung bei asymmetrischer Information, Finanzarchiv 49, S. 167 - 180.

Calabresi, G., 1968, Transaction Costs, Resource Allocation and Liability Rules: A Comment, Journal of Law and Economics 11, S. 67 - 73. 
Clarke, E. H., 1971, Multipart Pricing of Public Goods, Public Choice 8, S. 19 - 33.

Coase, R. H., 1960, The Problem of Social Cost, Journal of Law and Economics 3, S. 1- 44.

Dasgupta, P., P. Hammond und E. Maskin, 1979, The Implementation of Social Choice Rules, Review of Economic Studies 46, S. 185 - 216.

d'Aspremont, C., und L. A. Gerard-Varet, 1979, Incentives and Incomplete Information, Journal of Public Economics 11, S. 25 - 45.

Davis, O. A. und A. B. Whinston, 1962, Externalities, Welfare, and the Theory of Games, Journal of Political Economy 70, S. 241 - 262.

Demougin, D. und G. Illing, 1991, Regulating Environmental Quality under Asymmetric Information, CES Discussion Paper, No. 7, Munich.

Endres, A., 1977, Die Coase-Kontroverse, Zeitschrift für die gesamte Staatswissenschaft 133, S. 637 - 651 .

Farrell, J., 1987, Information and the Coase Theorem, Journal of Economic Perspectives 2, S. 113 - 129.

Feldman, A. M., 1982, Welfare Economics and Social Choice Theory, 2. Aufl., Boston u.a.O.

Fishelson, G., 1976, Emission Control Policies under Uncertainty, Journal of Environmental Economics and Management 3, S. 189 - 197.

Fudenberg, D. und J. Tirole, 1991, Game Theory, Cambridge u.a.O.

Gibbard, A., 1973, Manipulation of Voting Schemes: A General Result, Econometrica 41, S. 587 - 602.

Green, J. R. und J.-J. Laffont, 1977, Characterization of Satisfactory Mechanisms for the Revelation of Preferences for Public Goods, Econometrica 45, S. 427 - 438.

Green, J. R. und J.-J. Laffont, 1979, Incentives in Public Decision Making, Amsterdam u.a.O. 
Gresik, T. A. und M. A. Satterthwaite, 1989, The Rate at Which a Simple Market Converges to Efficiency as the Number of Traders Increases: An Asymptotic Result for Optimal Trading Mechanisms, Journal of Economic Theory 48, S. 304 - 332.

Groves, T., 1973, Incentives in Teams, Econometrica 41, S. 617 - 631.

Groves, T. und J. O. Ledyard, 1977a, Some Limitations of Demand Revealing Processes, Public Choice 29-2, S. 10 - 124.

Groves, T. und J. O. Ledyard, 1977b, Optimal Allocation of Public Goods: A Solution to the "Free Rider" Problem, Econometrica 45, S. 83 - 809.

Hayek, F. A., 1945, The Use of Knowledge in Society, American Economic Review 35 , S. $519-530$.

Harsanyi, J., 1967/1968, Games with Incomplete Information Played by Bayesian Players, Management Science 14, S. 159 - 182, 320 - 334 und 486 - 502.

Heuser, H., 1991, Lehrbuch der Analysis, 9. Aufl., Stuttgart.

Illing, G., 1992, Private Information as Transaction Costs: The Coase Theorem Revisited, Journal of Institutional and Theoretical Economics 148, S. 558 - 576.

Johansen, L., 1977, The Theory of Public Goods: Misplaced Emphasis?, Journal of Public Economics 7, S. 147 - 152.

Johansen, L., 1981, Review of: Green/Laffont, Incentives in Public Decision Making, Journal of Public Economics 16, S. 123 - 128.

Kreps, D. M., 1990, A Course in Microeconomic Theory, Princeton.

Kwerel, E., 1977, To Tell the Truth: Imperfect Information and Optimal Pollution Control, Review of Economic Studies 44, S. 595 - 601.

Laffont, J.-J. und E. Maskin, 1979, A Differential Approach to Expected Utility Maximizing Mechanisms, in: Laffont (Hrsg.), Aggregation and Revelation of Preferences, Amsterdam. 
Maskin, E. und J. Riley, 1984, Monopoly with Incomplete Information, Rand Journal of Economics 15, S. 171 - 196.

Maskin, E. und J. Tirole, 1990, The Principal-Agent Relationship with an Informed Principal: The Case of Private Values, Econometrica 58, S. 379 - 409.

Maskin, E. und J. Tirole, 1992, The Principal-Agent Relationship with an Informed Principal, II: Common Values, Econometrica 60, S. 1 - 42.

Mishan, E. J., 1971, The Postwar Literature on Externalities: An Interpretative Essay, Journal of Economic Literature 9, S. 1 - 28.

Mookherjee, D. und S. Reichelstein, 1992, Dominant Strategy Implementation of Bayesian Incentive Compatible Allocation Rules, Journal of Economic Theory 56, S. 378 - 399.

Muller, E. und M. A. Satterthwaite, 1985, Strategy-Proofness: The Existence of Dominant-Strategy Mechanisms, in: Hurwicz, Schmeidler und Sonnenschein (Hrsg.), Social Goals and Social Organization, Cambridge u.a.O.

Mumey, G. A., 1971, The "Coase Theorem": A Reexamination, Quarterly Journal of Economics 85, S. 718-723.

Myerson, R., 1979, Incentive Compatibility and the Bargaining Problem, Econometrica 47, S. 61 - 73.

Myerson, R., 1981, Optimal Auction Design, Mathematics of Operations Research 6, S. 58 - 73.

Myerson, R., 1983, Mechanism Design by an Informed Principal, Econometrica 51, S. $1767-1797$.

Myerson, R., 1985, Bayesian Equilibrium and Incentive-Compatibility: An Introduction, in: Hurwicz, Schmeidler und Sonnenschein (Hrsg.), Social Goals and Social Organization, Cambridge u.a.O.

Myerson, R. und M. A. Satterthwaite, 1983, Efficient Mechanisms for Bilateral Trading, Journal of Economic Theory 28, S. 265 - 281. 
Ng, Y.-K., 1979, Welfare Economics, London.

Pearce, D. W. und R. K. Turner, 1990, Economics of Natural Resources and the Environment, New York u.a.O.

Pigou, A. C., 1932, The Economics of Welfare, 4. Aufl., London.

Pommerehne, W., 1987, Präferenzen für öffentliche Güter, Tübingen.

Rasmusen, E., 1989, Games and Information, Oxford.

Repullo, R., 1986, On the Revelation Principle under Complete and Incomplete Information, in: K. Binmore und P. Dasgupta (Hrsg.), Economic Organizations as Games, Oxford u.a.O.

Richter, W. und W. Wiegard, 1993, Zwanzig Jahre "Neue Finanzwissenschaft", Teil I: Überblick und Theorie des Marktversagens, Zeitschrift für Wirtschafts- und Sozialwissenschaften 113, S. 169 - 224.

Rob, R., 1989, Pollution Claim Settlements under Private Information, Journal of Economic Theory 47, S. 307 - 333.

Roberts, M. J. und M. Spence, 1976, Effluent Charges and Licences under Uncertainty, Journal of Public Economics 5, S. 193 - 208.

Rubinstein, A., 1982, Perfect Equilibrium in a Bargaining Model, Econometrica 50, S. $97-111$.

Samuelson, W., 1985, A Comment on the Coase Theorem, in: A. E. Roth (Hrsg.), Game Theoretic Models of Bargaining, Cambridge.

Satterthwaite, M. A., 1975, Strategy-Proofness and Arrow's Conditions: Existence- and Correspondence-Theorems for Voting Procedures and Social Welfare Functions, Journal of Economic Theory 10, S. 187 - 217.

Schelling, T. C., 1956, An Essay on Bargaining, American Economic Review 46, S. $281-306$. 
Schweizer, U., 1988, Externalities and the Coase Theorem: Hypothesis or Result?, Journal of Institutional and Theoretical Economics 144, S. 245 - 266.

Shapiro, J. M. und D. R. Whitney, 1967, Elementary Analysis and Statistics, Columbus.

Sinn, H.-W., 1993, Pigou and Clarke Join Hands, Public Choice 75, S. 79 - 91.

Sinn, H.-W. und U. Schmoltzi, 1981, Eigentumsrechte, Kompensationsregeln und Marktmacht, Jahrbücher für Nationalökonomie und Statistik 196, S. 97 - 117.

Sutton, J.,1986, Non-Cooperative Bargaining Theory: An Introduction, Review of Economic Studies 53, S. 709 - 724.

Tideman, N. und G. Tullock, 1976, A New and Superior Process for Making Social Choices, Journal of Political Economy 84, S. 1145 - 1160.

Trockel, W., 1991, Über Informationsprobleme bei der Implementation von Mechanismen, Zeitschrift für Wirtschafts- und Sozialwissenschaften 111, S. 207 - 226.

Turvey, R., 1963, Divergences between Social Cost and Private Cost, Economica 30, S. 309-313.

Vickrey, W., 1961, Counterspeculation, Auctions and Competitive Sealed Tenders, Journal of Finance 16, S. 1 - 17.

Weimann, J., 1990, Umweltökonomik - Eine theorieorientierte Einführung, Berlin u.a.O.

Weitzmann, M. L., 1974, Prices vs. Quantities, Review of Economic Studies 41, S. 477 - 491 .

Wilson, R., 1985, Incentive Efficiency of Double Auctions, Econometrica 53, S. $1101-1115$.

Worcester, D. A., 1972, A Note on "The Postwar Literature on Externalities: An Interpretative Essay", Journal of Economic Literature 10, S. 57 - 59. 


\section{FINANZWISSENSCHAFTLICHE SCHRIFTEN}

Band 1 Werner Steden: Finanzpolitik und Einkommensverteilung. Ein Wachstums- und Konjunkturmodell der Bundesrepublik Deutschland. 1979.

Band 2 Rainer Hagemann: Kommunale Finanzplanung im föderativen Staat. 1976.

Band 3 Klaus Scherer: Maßstäbe zur Beurteilung von konjunkturellen Wirkungen des öffentlichen Haushalts. 1977.

Band 4 Brita Steinbach: "Formula Flexibility" - Kritische Analyse und Vergleich mit diskretionărer Konjunkturpolitik. 1977.

Band 5 Hans-Georg Petersen: Personelle Einkommensbesteuenung und Inflation. Eine theoretisch-empirische Analyse der Lohn- und veranlagten Einkommensteuer in der Bundesrepublik Deutschland. 1977.

Band 6 Friedemann Tetsch: Raumwirkungen des Finanzsystems der Bundesrepublik Deutschland. Eine Untersuchung der Auswirkungen der Finanzreform von 1969 auf die Einnahmenposition der untergeordneten Gebietskörperschaften und ihrer regionalpolitischen Zieladäquanz. 1978.

Band 7 Wilhelm Pfähler: Normative Theorie der fiskalischen Besteuerung. Ein methodologischer und theoretischer Beitrag zur Integration der normativen Besteuerungstheorie in der Wohlfahrtstheorie. 1978.

Band 8 Wolfgang Wiegard: Optimale Schattenpreise und Produktionsprogramme für öffentliche Unternehmen. Second-Best Modelle im finanzwirtschaftlichen Staatsbereich. 1978.

Band 9 Hans P. Fischer: Die Finanzierung des Umweltschutzes im Rahmen einer rationalen Umweltpolitik. 1978.

Band 10 Rainer Paulenz: Der Einsatz finanzpolitischer Instrumente in der Forschungs- und Entwicklungspolitik. 1978.

Band 11 Hans-Joachim Hauser: Verteilungswirkungen der Staatsverschuldung. Eine kreislauftheoretische Inzidenzbetrachtung. 1979.

Band 12 Gunnar Schwarting: Kommunale Investitionen. Theoretische und empirische Untersuchungen der Bestimmungsgrūnde kommunaler Investitionstătigkeit in NordrheinWestfalen 1965-1972. 1979.

Band 13 Hans-Joachim Conrad: Stadt-Umland-Wanderung und Finanzwirtschaft der Kernstădte. Amerikanische Erfahrungen, grundsătzliche Zusammenhănge und eine Fallstudie für das Ballungsgebiet Frankfurt am Main. 1980.

Band 14 Cay Folkers: Vermögensverteilung und staatliche Aktivităt. Zur Theorie distributiver Prozesse im Interventionsstaat. 1981.

Band 15 Helmut Fischer: US-amerikanische Exportförderung durch die DISC-Gesetzgebung. 1981.

Band 16 Günter Ott: Einkommensumverteilungen in der gesetzlichen Krankenversicherung. Eine quantitative Analyse. 1981.

Band 17 Johann Hermann von Oehsen: Optimale Besteuerung. (Optimal Taxation). 1982.

Band 18 Richard Kössler: Sozialversicherungsprinzip und Staatszuschūsse in der gesetzlichen Rentenversicherung. 1982.

Band 19 Hinrich Steffen: Zum Handlungs- und Entscheidungsspielraum der kommunalen Investitionspolitik in der Bundesrepublik Deutschland. 1983.

Band 20 Manfred Scheuer: Wirkungen einer Auslandsverschuldung des Staates bei flexiblen Wechselkursen. 1983. 
Band 21 Christian Schiller: Staatsausgaben und crowding-out-Effekte. Zur Effizienz einer Finanzpolitik keynesianischer Provenienz. 1983.

Band 22 Hannelore Weck: Schattenwirtschaft: Eine Möglichkeit zur Einschränkung der öffentlichen Verwaltung? Eine ökonomische Analyse. 1983.

Band 23 Wolfgang Schmitt: Steuern als Mittel der Einkommenspolitik. Eine Ergänzung der Stabilitätspolitik? 1984.

Band 24 Wolfgang Laux: Erhöhung staatswirtschaftlicher Effizienz durch budgetäre Selbstbeschränkung? Zur Idee einer verfassungsmäßig verankerten Ausgabengrenze. 1984.

Band 25 Brita Steinbach-van der Veen: Steuerinzidenz. Methodologische Grundlagen und empirisch-statistische Probleme von Länderstudien. 1985.

Band 26 Albert Peters: Ökonomische Kriterien für eine Aufgabenverteilung in der Marktwirtschaft. Eine deskriptive und normative Betrachtung für den Allokationsbereich. 1985.

Band 27 Achim Zeidler: Möglichkeiten zur Fortsetzung der Gemeindefinanzreform. Eine theoretische und empirische Analyse. 1985.

Band 28 Peter Bartsch: Zur Theorie der längerfristigen Wirkungen 'expansiver' Fiskalpolitik. Eine dynamische Analyse unter besonderer Berücksichtigung der staatlichen Budgetbeschränkung und ausgewählter Möglichkeiten der öffentlichen Defizitfinanzierung. 1986.

Band 29 Konrad Beiwinkel: Wehrgerechtigkeit als finanzpolitisches Verteilungsproblem. Möglichkeiten einer Kompensation von Wehrungerechtigkeit durch monetäre Transfers. 1986.

Band 30 Wolfgang Kitterer: Effizienz- und Verteilungswirkungen des Steuersystems. 1986.

Band 31 Heinz Dieter Hessler: Theorie und Politik der Personalsteuern. Eine Kritik ihrer Einkommens- und Vermögensbegriffe mit Blick auf die Leistungsfähigkeitstheorie. 1994.

Band 32 Wolfgang Scherf: Die beschäftigungspolitische und fiskalische Problematik der Arbeitgeberbeiträge zur Rentenversicherung. Eine Auseinandersetzung mit der Kritik an der lohnbezogenen Beitragsbemessung. 1987.

Band 33 Andreas Mästle: Die Steuerunion. Probleme der Harmonisierung spezifischer Gütersteuern. 1987.

Band 34 Günter Ott: Internationale Verteilungswirkungen im Finanzausgleich der Europäischen Gemeinschaften. 1987.

Band 35 Heinz Haller: Zur Frage der zweckmäßigen Gestalt gemeindlicher Steuern. Ein Diskussionsbeitrag zur Gemeindesteuerreform. 1987.

Band 36 Thomas Kuhn: Schlüsselzuweisungen und fiskalische Ungleichheit. Eine theoretische Analyse der Verteilung von Schlüsselzuweisungen an Kommunen. 1988.

Band 37 Walter Hahn: Steuerpolitische Willensbildungsprozesse in der Europäischen Gemeinschaft. Das Beispiel der Umsatzssteuer-Harmonisierung. 1988.

Band 38 Ulrike Hardt: Kommunale Finanzkraft. Die Problematik einer objektiven Bestimmung kommunaler Einnahmemöglichkeiten in der gemeindlichen Haushaltsplanung und im kommunalen Finanzausgleich. 1988.

Band 39 Jochen Michaelis: Optimale Finanzpolitik im Modell überlappender Generationen. 1989.

Band 40 Bernd Raffelhüschen: Anreizwirkungen der sozialen Alterssicherung. Eine dynamische Simulationsanalyse. 1989.

Band 41 Berend Diekmann: Die Anleihe- und Darlehenstransaktionen der Europäischen Gemeinschaften. 1990.

Band 42 Helmut Kaiser: Konsumnachfrage, Arbeitsangebot und optimale Haushaltsbesteuerung. Theoretische Ergebnisse und mikroökonometrische Simulation für die Bundesrepublik Deutschland. 1990. 
Band 43 Rüdiger von Kleist: Das Gramm-Rudman-Hollings-Gesetz. Ein gescheiterter Versuch der Haushaltskonsolidierung. 1991.

Band 44 Rolf Hagedorn: Steuerhinterziehung und Finanzpolitik. Ein theoretischer Beitrag unter besonderer Berücksichtigung der Hinterziehung von Zinserträgen. 1991.

Band 45 Cornelia S. Behrens: Intertemporale Verteilungswirkungen in der gesetzlichen Krankenversicherung der Bundesrepublik Deutschland. 1991.

Band 46 Peter Saile: Ein ökonomischer Ansatz der Theorie der intermediären Finanzgewalten Die Kirchen als Parafisci. 1992.

Band 47 Peter Gottfried: Die verdeckten Effizienzwirkungen der Umsatzsteuer. Eine empirische allgemeine Gleichgewichtsanalyse. 1992.

Band 48 Andreas Burger: Umweltorientierte Beschäftigungsprogramme. Eine Effizienzanalyse am Beispiel des "Sondervermögens Arbeit und Umwelt". 1992.

Band 49 Jeanette Malchow: Die Zuordnung verteilungspolitischer Kompetenzen in der Europäischen Gemeinschaft. Eine Untersuchung aufgrund einer Fortentwicklung der ökonomischen Theorie des Föderalismus. 1992.

Band 50 Barbara Seidel: Die Einbindung der Bundesrepublik Deutschland in die Europäischen Gemeinschaften als Problem des Finanzausgleichs. 1992.

Band 51 Ralph Wiechers: Markt und Macht im Rundfunk. Zur Stellung der öffentlich-rechtlichen Rundfunkanstalten im dualen Rundfunksystem der Bundesrepublik Deutschland. 1992.

Band 52 Klaus Eckhardt: Probleme einer Umweltpolitik mit Abgaben. 1993.

Band 53 Oliver Schwarzkopf: Die Problematik unterschiedlicher Körperschaftsteuersysteme innerhalb der EG. 1993.

Band 54 Thorsten Giersch: Bergson-Wohlfahrtsfunktion und normative Ökonomie. 1993.

Band 55 Li-Fang Chou: Selbstbeteiligung bei Arzneimitteln aus ordnungspolitischer Sicht. Das Beispiel der Bundesrepublik Deutschland. 1993.

Band 56 Harald Schlee: Einkommensteuerliche Behandlung von Transferzahlungen. Zur Neuordnung der Familienbesteuerung sowie der Besteuerung von Versicherungsleistungen und Sozialtransfers. 1994.

Band 57 Alexander Spermann: Kommunales Krisenmanagement. Reaktionen baden-württembergischer Stadtkreise auf steigende Sozialhilfekosten und Einnahmenausfälle (198092). 1993.

Band 58 Otto Roloff / Sibylle Brander / Ingo Barens / Claudia Wesselbaum: Direktinvestitionen und internationale Steuerkonkurrenz. 1994.

Band 59 Claudia Wesselbaum-Neugebauer: Intemationale Steuerbelastungsvergleiche. 1994.

Band 60 Stephanie Miera: Kommunales Finanzsystem und Bevölkerungsentwicklung. Eine Analyse des kommunalen Finanzsystems vor dem Hintergrund der sich abzeichnenden Bevölkerungsentwicklung am Beispiel Niedersachsens unter besonderer Berūcksichtigung des Landkreises Wolfenbüttel und seiner Gemeinden. 1994.

Band 61 Wolfgang Scherf: Die Bedeutung des kaldorianischen Verteilungsmechanismus für die gesamtwirtschaftlichen Wirkungen der staatlichen Neuverschuldung. 1994.

Band 62 Rainer Volk: Vergleich der Vergünstigungseffekte der verschiedenen investitionsfördernden Maßnahmen. 1994.

Band 63 Hans-Georg Napp: Kommunale Finanzautonomie und ihre Bedeutung für eine effiziente lokale Finanzwirtschaft. 1994. 2., unverảnderte Auflage 1994.

Band 64 Bernd Rahmann / Uwe Steinborn / Gūnter Vornholz: Empirische Analyse der Autonomie lokaler Finanzwirtschaften in der Europäischen Gemeinschaft. 1994. 
Band 65 Carsten Kühl: Strategien zur Finanzierung der Altlastensanierung. 1994.

Band 66 Stephan Boll: Intergenerationale Umverteilungswirkungen der Fiskalpolitik in der Bundesrepublik Deutschland. Ein Ansatz mit Hilfe des Generational Accounting. 1994.

Band 68 Christian Haslbeck: Zentrale versus dezentrale Internalisierung externer Effekte bei unvollständiger Information. 1995. 


\title{
Jan Klingele
}

\section{Die Entwicklung der multinationalen Unternehmen aus Sicht der Internalisierungstheorie}

\author{
Frankfurt/M., Bern, New York, Paris, 1991. 399 S., 9 Abb., 3 Tab. \\ Europäische Hochschulschriften: Reihe 5, \\ Volks- und Betriebswirtschaft. Bd. 1169 \\ ISBN 3-631-43688-2 br. DM 99.--*
}

Die Bedeutung der multinationalen Unternehmen und die internationale Verflechtung der Wirtschaft ist in den letzten Jahrzehnten rasch angestiegen. In diesem Zusammenhang treten für Wirtschaft und Politik bedeutsame Fragen auf, welche die klassischen Theorien der Volks- und Betriebswirtschaftslehre nicht beantworten können. Hierfür ist die im englischen Sprachraum entwickelte Internalisierungstheorie hilfreich, die in diesem Buch vorgestellt, überprüft und weiterentwickelt wird. Neben der theoretischen Erklärung von Verhaltensweisen und Strategien der multinationalen Unternehmen wird eine Fülle von aktuellen empirischen Erkenntnissen über die international tätigen Firmen präsentiert.

Aus dem Inhalt. Bedeutung und Geschichte der multinationalen Unternehmen - Ungeklärte Probleme der klassischen Theorien - Grundlagen der Internalisierungstheorie - Prüfung und Weiterentwicklung der Internalisierungstheorie - Überblick über den derzeitigen empirischen Stand der Kenntnis - Eigenschaften und Verhaltensweisen der multinationalen Unternehmen - Dynamik der internationalen Unternehmensentwicklung

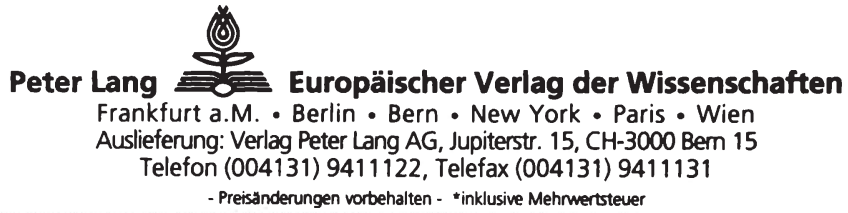


Christian Haslbeck - 978-3-631-75257-9

Downloaded from PubFactory at 01/11/2019 06:37:37AM

via free access 